Supporting Information for

\title{
Reactive Rigid-Rod Organometallic Polymers Involving Linear Triplatinum Units Connected by $\pi$-Conjugated Bisisocyanides
}

\author{
Tomoaki Tanase, ${ }^{\dagger}, *$ Eri Goto, ${ }^{\dagger}$ Rowshan A. Begum, ${ }^{\dagger}$ Makiko Hamaguchi, ${ }^{\dagger}$ \\ Shuzhong Zhan, ${ }^{\dagger}$ Masayasu Iida, ${ }^{+}$and Ken Sakai \\ †Department of Chemistry, Faculty of Science, Nara Women's University, \\ Kitauoya-higashi-machi, Nara 630-8285, Japan \\ $\ddagger$ Department of Applied Chemistry, Faculty of Engineering, \\ Tokyo University of Science, Kagurazaka 1-3, Shinjuku-ku, \\ Tokyo 162-8601, Japan
}

Table S1-1. Crystallographic and Experimental Data for $2 \cdot 2\left(\mathrm{CH}_{3}\right)_{2} \mathrm{CO}$

Table S1-2. Final Positional Parameters and $B_{\text {eq }}$ for $2 \cdot 2\left(\mathrm{CH}_{3}\right)_{2} \mathrm{CO}$

Table S1-3. Final Anisotropic Thermal Parameters for $2 \cdot 2\left(\mathrm{CH}_{3}\right)_{2} \mathrm{CO}$

Table S1-4. Bond Lengths for Non-hydrogen Atoms of $2 \cdot 2\left(\mathrm{CH}_{3}\right)_{2} \mathrm{CO}$

Table S1-5. Bond Angles for Non-hydrogen Atoms of $2 \cdot 2\left(\mathrm{CH}_{3}\right)_{2} \mathrm{CO}$

Table S2-1. Crystallographic and Experimental Data for $3 \cdot 2.5 \mathrm{CH}_{2} \mathrm{Cl}_{2}$

Table S2-2. Final Positional Parameters and $B$ eq for $3 \cdot 2.5 \mathrm{CH}_{2} \mathrm{Cl}_{2}$

Table S2-3. Final Anisotropic Thermal Parameters for $3 \cdot 2.5 \mathrm{CH}_{2} \mathrm{Cl}_{2}$

Table S2-4. Bond Lengths for Non-hydrogen Atoms of $3 \cdot 2.5 \mathrm{CH}_{2} \mathrm{Cl}_{2}$

Table S2-5. Bond Angles for Non-hydrogen Atoms of $3 \cdot 2.5 \mathrm{CH}_{2} \mathrm{Cl}_{2}$

Table S3-1. Crystallographic and Experimental Data for 4

Table S3-2. Final Positional Parameters and $B_{\text {eq }}$ for 4

Table S3-3. Final Anisotropic Thermal Parameters for 4

Table S3-4. Bond Lengths for Non-hydrogen Atoms of 4

Table S3-5. Bond Angles for Non-hydrogen Atoms of 4

Table S4-1. Crystallographic and Experimental Data for $6 \mathrm{a} \cdot\left(\mathrm{CH}_{3}\right)_{2} \mathrm{CO} \cdot \mathrm{Et}_{2} \mathrm{O}$

Table S4-2. Final Positional Parameters and $B_{\text {eq }}$ for $\mathbf{6 a} \cdot\left(\mathrm{CH}_{3}\right)_{2} \mathrm{CO} \cdot \mathrm{Et}_{2} \mathrm{O}$

Table S4-3. Final Anisotropic Thermal Parameters for $6 \mathrm{a} \cdot\left(\mathrm{CH}_{3}\right)_{2} \mathrm{CO} \cdot \mathrm{Et}_{2} \mathrm{O}$

Table S4-4. Bond Lengths for Non-hydrogen Atoms of $6 \mathrm{a} \cdot\left(\mathrm{CH}_{3}\right)_{2} \mathrm{CO} \cdot \mathrm{Et}_{2} \mathrm{O}$

Table S4-5. Bond Angles for Non-hydrogen Atoms of $6 \mathrm{a} \cdot\left(\mathrm{CH}_{3}\right)_{2} \mathrm{CO} \cdot \mathrm{Et}_{2} \mathrm{O}$

Table S5-1. Crystallographic and Experimental Data for $\mathbf{6 c} \cdot 2 \mathrm{CH}_{3} \mathrm{CN}$

Table S5-2. Final Positional Parameters and $B_{\text {eq }}$ for $6 \mathrm{c} \cdot 2 \mathrm{CH}_{3} \mathrm{CN}$

Table S5-3. Final Anisotropic Thermal Parameters for $\mathbf{6 c} \cdot 2 \mathrm{CH}_{3} \mathrm{CN}$

Table S5-4. Bond Lengths for Non-hydrogen Atoms of $6 \mathrm{c} \cdot 2 \mathrm{CH}_{3} \mathrm{CN}$

Table S5-5. Bond Angles for Non-hydrogen Atoms of $6 \mathrm{c} \cdot 2 \mathrm{CH}_{3} \mathrm{CN}$

Table S6-1. Crystallographic and Experimental Data for $7 \mathbf{a} \cdot 3 \mathrm{CH}_{2} \mathrm{Cl}_{2}$

Table S6-2. Final Positional Parameters and $B_{\text {eq }}$ for $7 \mathbf{a} \cdot 3 \mathrm{CH}_{2} \mathrm{Cl}_{2}$

Table S6-3. Final Anisotropic Thermal Parameters for $7 \mathbf{a} \cdot 3 \mathrm{CH}_{2} \mathrm{Cl}_{2}$

Table S6-4. Bond Lengths for Non-hydrogen Atoms of $7 \mathbf{a} \cdot 3 \mathrm{CH}_{2} \mathrm{Cl}_{2}$

Table S6-5. Bond Angles for Non-hydrogen Atoms of $7 \mathbf{a} \cdot 3 \mathrm{CH}_{2} \mathrm{Cl}_{2}$

Table S7-1. Crystallographic and Experimental Data for $9 \mathbf{b} \cdot 5 \mathrm{CH}_{2} \mathrm{Cl}_{2}$

Table S7-2. Final Positional Parameters and $B_{\text {eq }}$ for $9 \mathbf{b} \cdot 5 \mathrm{CH}_{2} \mathrm{Cl}_{2}$

Table S7-3. Final Anisotropic Thermal Parameters for $9 \mathbf{b} \cdot 5 \mathrm{CH}_{2} \mathrm{Cl}_{2}$ 
Table S7-4. Bond Lengths for Non-hydrogen Atoms of $9 \mathbf{b} \cdot 5 \mathrm{CH}_{2} \mathrm{Cl}_{2}$

Table S7-5. Bond Angles for Non-hydrogen Atoms of $9 \mathbf{b} \cdot 5 \mathrm{CH}_{2} \mathrm{Cl}_{2}$

Table S8-1. Crystallographic and Experimental Data for $10 \mathrm{a} \cdot \mathrm{CH}_{2} \mathrm{Cl}_{2} \cdot 3 \mathrm{H}_{2} \mathrm{O}$

Table S8-2. Final Positional Parameters and $B_{\text {eq }}$ for $10 \mathrm{a} \cdot \mathrm{CH}_{2} \mathrm{Cl}_{2} \cdot 3 \mathrm{H}_{2} \mathrm{O}$

Table S8-3. Final Anisotropic Thermal Parameters for $10 \mathrm{a} \cdot \mathrm{CH}_{2} \mathrm{Cl}_{2} \cdot 3 \mathrm{H}_{2} \mathrm{O}$

Table S8-4. Bond Lengths for Non-hydrogen Atoms of $10 \mathrm{a} \cdot \mathrm{CH}_{2} \mathrm{Cl}_{2} \cdot 3 \mathrm{H}_{2} \mathrm{O}$

Table S8-5. Bond Angles for Non-hydrogen Atoms of $10 \mathrm{a} \cdot \mathrm{CH}_{2} \mathrm{Cl}_{2} \cdot 3 \mathrm{H}_{2} \mathrm{O}$

Table S9-1. Crystallographic and Experimental Data for 11a $\cdot\left(\mathrm{CH}_{3}\right)_{2} \mathrm{CO}$

Table S9-2. Final Positional Parameters and $B_{\text {eq }}$ for 11a $\cdot\left(\mathrm{CH}_{3}\right)_{2} \mathrm{CO}$

Table S9-3. Final Anisotropic Thermal Parameters for 11a. $\left(\mathrm{CH}_{3}\right)_{2} \mathrm{CO}$

Table S9-4. Bond Lengths for Non-hydrogen Atoms of 11a $\left(\mathrm{CH}_{3}\right)_{2} \mathrm{CO}$

Table S9-5. Bond Angles for Non-hydrogen Atoms of 11a $\cdot\left(\mathrm{CH}_{3}\right)_{2} \mathrm{CO}$

Figure S1. ORTEP Plot for Complex 2

Figure S2. ORTEP Plot for Complex 4

Figure S3-1. Raw EXAFS data, $k^{3} \chi(k)$

Figure S3-2 Fourier Transforms of $k^{3} \chi(k)$

Figure S3-3 Curve-fitting Results

Figure S4. UV-Vis Spectra of Complexes 1-5

Figure S5. ORTEP Plot for Complex 6a

Figure S6. ORTEP Plot for Complex 6c

Figure S7. MO Interaction Diagram for $\left[\mathrm{Pt}_{3}(\mu-\mathrm{NO})_{2}\left(\mathrm{PH}_{3}\right)_{6}(\mathrm{CNH})_{2}\right]^{4+}(\mathrm{C})$ from the Fragments $\left[\mathrm{Pt}_{3}\left(\mathrm{PH}_{3}\right)_{6}(\mathrm{CNH})_{2}\right]^{2+}(\mathrm{C} 1)$ and $\left[(\mathrm{NO})_{2}\right]^{2+}(\mathrm{C} 2)$ on the Basis of EHMO Calculations and CACAO Fragment Analysis.

Figure S8. Electronic Absorption Spectra of the Polymer 13 and of the Polymer 13 with Excess of $\mathrm{Et}_{3} \mathrm{~N}$.

Equation 1. Theoretical Expression of EXAFS Oscillation. 
Table S1-1. Crystallographic and Experimental Data for $2 \cdot 2\left(\mathrm{CH}_{3}\right)_{2} \mathrm{CO}$

\begin{tabular}{|c|c|}
\hline formula & $\mathrm{C}_{90} \mathrm{H}_{92} \mathrm{~N}_{2} \mathrm{O}_{2} \mathrm{P}_{8} \mathrm{~F}_{12} \mathrm{Pt}_{3}$ \\
\hline formula weight & 2294.77 \\
\hline color & orange \\
\hline size, $\mathrm{mm}$ & $0.40 \times 0.38 \times 0.10$ \\
\hline cryst. system & monoclinic \\
\hline space group & $P 21 / a($ No. 14$)$ \\
\hline no. refs used for cell determination & $25\left(20<2 \theta<30^{\circ}\right)$ \\
\hline \multirow[t]{2}{*}{ lattice const. } & $\begin{array}{l}a=14.133(4) \AA \\
b=22.108(6) \AA \\
c=29.505(8) \AA \\
\beta=99.70(3) \circ\end{array}$ \\
\hline & $V=9087(4) \AA^{3}$ \\
\hline Z & 4 \\
\hline$D_{\text {calc }}, \mathrm{g} \mathrm{cm}^{-3}$ & 1.677 \\
\hline$F(000)$ & 4496 \\
\hline$\mu(\operatorname{Mo} K \alpha), \mathrm{cm}^{-1}$ & 48.01 (trans. $0.66 \sim 1.00)$ \\
\hline \multicolumn{2}{|c|}{ An absorption correction was applied ( $\psi$-scan method). } \\
\hline diffractometer & Rigaku AFC7R (50 kV 200 mA) \\
\hline radiation & $\operatorname{Mo} K \alpha(\lambda=0.71069 \AA)$ \\
\hline monochromator & graphite \\
\hline temp, ${ }^{\circ} \mathrm{C}$ & -118 \\
\hline $2 \theta$ range, $^{\circ}$ & $4<2 \theta<45$ \\
\hline$h, k$, l range & $+\mathrm{h},+\mathrm{k}, \pm \mathrm{l}$ \\
\hline scan method & $\omega-2 \theta$ \\
\hline scan speed, ${ }^{\circ} \min ^{-1}$ & 8 \\
\hline scan width & $0.89+0.30 \tan \theta$ \\
\hline std. reflections & 3 every 150 refs \\
\hline solution & Direct Methods (SIR92) \\
\hline \multirow[t]{2}{*}{ refinement } & full-matrix least-squares \\
\hline & $\begin{array}{l}\text { All non-hydrogen atoms were refined } \\
\text { with aniotropic temperature factors. }\end{array}$ \\
\hline hydrogen atoms & $\begin{array}{l}\text { All } \mathrm{H} \text { atoms except for solvent molecules } \\
\text { were calcd with } \mathrm{C}-\mathrm{H}=0.95 \AA \text {, and were not refined. }\end{array}$ \\
\hline function minimized & $\Sigma w\left(\left|F_{o}\right|-\left|F_{c}\right|\right)^{2}$ \\
\hline weighting scheme & $1 / \sigma^{2}\left(F_{o}\right)$ \\
\hline anomalous dispersion & all non-hydrogen atoms \\
\hline no. unique reflections & 11775 \\
\hline$R_{\text {int }}$ & 0.056 \\
\hline no. obsd. unique reflections & $7748(I>2 \sigma(I))$ \\
\hline$p$-factor & 0.05 \\
\hline no. variables & 1055 \\
\hline data / param ratio & 7.34 \\
\hline$R^{\mathrm{a}}$ & 0.051 \\
\hline$R_{\mathrm{W}} \mathrm{b}$ & 0.054 \\
\hline $\mathrm{GOF}^{\mathrm{C}}$ & 1.19 \\
\hline$\rho_{\max }, \mathrm{e}^{-3}$ & $1.10,-1.50$ \\
\hline
\end{tabular}

a $R=\Sigma|| F_{\mathrm{O}}|-| F_{\mathrm{C}}|| / \Sigma\left|F_{\mathrm{O}}\right|$.

$\mathrm{b}_{R_{\mathrm{W}}}=\left[\Sigma w\left(\left|F_{\mathrm{O}}\right|-\left|F_{\mathrm{C}}\right|\right)^{2} / \Sigma w\left|F_{\mathrm{O}}\right|^{2}\right]^{1 / 2}\left(w=1 / \sigma^{2}\left(F_{\mathrm{O}}\right)\right)$.

${ }^{c} \mathrm{GOF}=\left[\Sigma w\left(\left|F_{\mathrm{O}}\right|-\left|F_{\mathrm{C}}\right|\right)^{2} /\left(N_{\mathrm{O}}-N_{\mathrm{p}}\right]^{1 / 2}\left(N_{\mathrm{O}}=\right.\right.$ no. data, $N_{\mathrm{p}}=$ no. variables $)$.

Table S1-2. Final Positional Parameters and $\mathrm{B}_{\mathrm{eq}}$ for $2 \cdot 2\left(\mathrm{CH}_{3}\right)_{2} \mathrm{CO}^{\mathrm{a}, \mathrm{b}, \mathrm{c}}$

\begin{tabular}{ccccc}
\hline atom & $x$ & $y$ & $z$ & $B_{\text {eq }}$ \\
\hline $\operatorname{Pt}(1)$ & $0.42278(4)$ & $0.09357(3)$ & $0.31810(2)$ & $2.29(1)$
\end{tabular}




\begin{tabular}{|c|c|c|c|c|}
\hline $\operatorname{Pt}(2)$ & $0.46494(4)$ & $0.02067(3)$ & $0.25025(2)$ & $2.12(1)$ \\
\hline $\operatorname{Pt}(3)$ & $0.50678(4)$ & $-0.05017(3)$ & $0.18155(2)$ & $2.18(1)$ \\
\hline $\mathrm{P}(1)$ & $0.3107(3)$ & $0.1380(2)$ & $0.2639(1)$ & $2.49(10)$ \\
\hline $\mathrm{P}(2)$ & $0.3146(3)$ & $0.0271(2)$ & $0.2106(1)$ & 2.31(9) \\
\hline $\mathrm{P}(3)$ & $0.4053(3)$ & $0.0082(2)$ & $0.1303(1)$ & $2.62(10)$ \\
\hline $\mathrm{P}(4)$ & $0.5359(3)$ & $0.0372(2)$ & $0.3629(1)$ & $2.65(10)$ \\
\hline$P(5)$ & $0.6213(3)$ & $0.0149(2)$ & $0.2819(1)$ & $2.45(9)$ \\
\hline$P(6)$ & $0.6116(3)$ & $-0.1040(2)$ & $0.2345(1)$ & $2.12(9)$ \\
\hline $\mathrm{P}(11)$ & $0.8784(3)$ & $-0.1215(2)$ & $0.3967(2)$ & $4.3(1)$ \\
\hline $\mathrm{P}(12)$ & $0.0603(4)$ & $0.1275(3)$ & $0.0783(2)$ & $5.8(2)$ \\
\hline $\mathrm{F}(11)$ & $0.8349(10)$ & $-0.0564(6)$ & $0.3885(6)$ & $11.2(5)$ \\
\hline $\mathrm{F}(12)$ & $0.879(1)$ & $-0.1209(9)$ & $0.4471(4)$ & $12.8(6)$ \\
\hline $\mathrm{F}(13)$ & $0.7766(9)$ & $-0.1466(7)$ & $0.3861(5)$ & $11.0(5)$ \\
\hline $\mathrm{F}(14)$ & $0.9778(9)$ & $-0.0950(8)$ & $0.4072(8)$ & $16.8(8)$ \\
\hline $\mathrm{F}(15)$ & $0.874(1)$ & $-0.123(1)$ & $0.3436(5)$ & $16.7(8)$ \\
\hline $\mathrm{F}(16)$ & $0.920(1)$ & $-0.1852(7)$ & $0.4013(6)$ & $14.9(6)$ \\
\hline $\mathrm{F}(21)$ & $0.0709(10)$ & $0.1962(6)$ & $0.0854(6)$ & $10.2(5)$ \\
\hline$F(22)$ & $-0.0514(8)$ & $0.1327(7)$ & $0.0787(4)$ & $8.3(4)$ \\
\hline $\mathrm{F}(23)$ & $0.0777(9)$ & $0.1182(7)$ & $0.1317(4)$ & $9.5(5)$ \\
\hline$F(24)$ & $0.1737(8)$ & $0.1233(6)$ & $0.0773(4)$ & $8.0(4)$ \\
\hline$F(25)$ & $0.053(1)$ & $0.0574(6)$ & $0.0740(6)$ & $12.7(6)$ \\
\hline $\mathrm{F}(26)$ & $0.0437(9)$ & $0.1321(7)$ & $0.0252(4)$ & $9.5(4)$ \\
\hline $\mathrm{O}(701)$ & $0.8503(10)$ & $0.1046(8)$ & $0.3958(6)$ & $8.3(5)$ \\
\hline $\mathrm{O}(702)$ & $0.670(1)$ & $0.4770(10)$ & $0.3261(6)$ & $11.0(7)$ \\
\hline $\mathrm{N}(1)$ & $0.3832(9)$ & $0.1744(6)$ & $0.4006(5)$ & $3.4(4)$ \\
\hline $\mathrm{N}(2)$ & $0.5676(9)$ & $-0.1238(6)$ & $0.1005(4)$ & $3.5(4)$ \\
\hline$C(1)$ & $0.395(1)$ & $0.1451(7)$ & $0.3689(6)$ & $3.0(4)$ \\
\hline$C(2)$ & $0.5430(10)$ & $-0.0980(7)$ & $0.1306(5)$ & $2.6(4)$ \\
\hline$C(3)$ & $0.234(1)$ & $0.0800(7)$ & $0.2321(5)$ & $3.1(4)$ \\
\hline$C(4)$ & $0.325(1)$ & $0.0579(7)$ & $0.1547(5)$ & $2.9(4)$ \\
\hline$C(5)$ & $0.6504(10)$ & $0.0397(7)$ & $0.3413(5)$ & $2.9(4)$ \\
\hline$C(6)$ & $0.6603(10)$ & $-0.0644(6)$ & $0.2873(5)$ & $2.3(4)$ \\
\hline$C(11)$ & $0.367(1)$ & $0.2030(7)$ & $0.4407(5)$ & $2.6(4)$ \\
\hline$C(12)$ & $0.292(1)$ & $0.1820(8)$ & $0.4624(5)$ & $3.9(5)$ \\
\hline$C(13)$ & $0.280(1)$ & $0.2108(9)$ & $0.5024(6)$ & $4.4(5)$ \\
\hline$C(14)$ & $0.338(1)$ & $0.2574(8)$ & $0.5207(6)$ & $4.2(5)$ \\
\hline$C(15)$ & $0.411(1)$ & $0.2768(8)$ & $0.4986(6)$ & $5.0(5)$ \\
\hline$C(16)$ & $0.427(1)$ & $0.2497(8)$ & $0.4577(6)$ & $4.1(5)$ \\
\hline$C(17)$ & $0.233(1)$ & $0.132(1)$ & $0.4412(7)$ & $6.5(6)$ \\
\hline$C(18)$ & $0.318(1)$ & $0.2863(9)$ & $0.5650(6)$ & $5.9(6)$ \\
\hline$C(19)$ & $0.507(1)$ & $0.2700(9)$ & $0.4336(7)$ & $6.3(6)$ \\
\hline$C(21)$ & $0.605(1)$ & $-0.1544(8)$ & $0.0673(6)$ & $3.6(5)$ \\
\hline$C(22)$ & $0.686(1)$ & $-0.1298(8)$ & $0.0518(5)$ & $3.3(4)$ \\
\hline$C(23)$ & $0.725(1)$ & $-0.161(1)$ & $0.0185(8)$ & $5.9(6)$ \\
\hline$C(24)$ & $0.685(2)$ & $-0.213(1)$ & $0.0023(7)$ & $6.4(7)$ \\
\hline$C(25)$ & $0.605(2)$ & $-0.2384(9)$ & $0.0168(6)$ & $5.6(6)$ \\
\hline$C(26)$ & $0.564(1)$ & $-0.2093(8)$ & $0.0509(6)$ & $4.0(5)$ \\
\hline$C(27)$ & $0.728(1)$ & $-0.0716(9)$ & $0.0699(8)$ & $6.0(6)$ \\
\hline$C(28)$ & $0.725(2)$ & $-0.249(1)$ & $-0.0361(8)$ & $10.4(9)$ \\
\hline$C(29)$ & $0.477(1)$ & $-0.2331(9)$ & $0.0676(7)$ & $6.1(6)$ \\
\hline$C(111)$ & $0.230(1)$ & $0.1899(7)$ & $0.2869(6)$ & $3.2(4)$ \\
\hline$C(112)$ & $0.140(1)$ & $0.1720(8)$ & $0.2927(7)$ & $4.8(5)$ \\
\hline$C(113)$ & $0.084(1)$ & $0.2137(9)$ & $0.3148(7)$ & $5.5(6)$ \\
\hline$C(114)$ & $0.120(1)$ & $0.2690(9)$ & $0.3291(7)$ & $4.7(5)$ \\
\hline$C(115)$ & $0.209(2)$ & $0.2856(8)$ & $0.3233(7)$ & $5.9(6)$ \\
\hline$C(116)$ & $0.264(1)$ & $0.2456(8)$ & $0.3017(7)$ & $4.9(5)$ \\
\hline$C(121)$ & $0.354(1)$ & $0.1838(7)$ & $0.2222(5)$ & $3.2(4)$ \\
\hline$C(122)$ & $0.292(2)$ & $0.2151(8)$ & $0.1875(6)$ & $5.3(6)$ \\
\hline
\end{tabular}




\begin{tabular}{|c|c|c|c|c|}
\hline$C(123)$ & $0.328(2)$ & $0.2525(9)$ & $0.1578(8)$ & $6.9(7)$ \\
\hline$C(124)$ & $0.427(3)$ & $0.259(1)$ & $0.161(1)$ & $9(1)$ \\
\hline C(125) & $0.486(2)$ & $0.230(1)$ & $0.1932(10)$ & $8.4(9)$ \\
\hline$C(126)$ & $0.454(1)$ & $0.1915(8)$ & $0.2244(6)$ & $4.6(5)$ \\
\hline$C(211)$ & $0.2415(9)$ & $-0.0401(6)$ & $0.1988(5)$ & $2.3(4)$ \\
\hline$C(212)$ & $0.159(1)$ & $-0.0425(7)$ & $0.1663(5)$ & $3.0(4)$ \\
\hline$C(213)$ & $0.108(1)$ & $-0.0964(9)$ & $0.1578(6)$ & $4.0(5)$ \\
\hline$C(214)$ & $0.134(1)$ & $-0.1465(8)$ & $0.1838(7)$ & $4.6(5)$ \\
\hline$C(215)$ & $0.217(1)$ & $-0.1450(8)$ & $0.2162(7)$ & $4.8(5)$ \\
\hline$C(216)$ & $0.270(1)$ & $-0.0933(7)$ & $0.2233(6)$ & $3.3(4)$ \\
\hline$C(311)$ & $0.325(1)$ & $-0.0353(7)$ & $0.0887(5)$ & $2.8(4)$ \\
\hline$C(312)$ & $0.271(1)$ & $-0.0067(8)$ & $0.0519(5)$ & $4.2(5)$ \\
\hline$C(313)$ & $0.204(1)$ & $-0.037(1)$ & $0.0213(6)$ & $5.6(6)$ \\
\hline$C(314)$ & $0.189(1)$ & $-0.099(1)$ & $0.0268(6)$ & $4.7(5)$ \\
\hline$C(315)$ & $0.245(1)$ & $-0.1286(9)$ & $0.0652(6)$ & $4.5(5)$ \\
\hline$C(316)$ & $0.310(1)$ & $-0.0984(9)$ & $0.0938(6)$ & $4.0(5)$ \\
\hline C(321) & $0.470(1)$ & $0.0573(10)$ & $0.0957(6)$ & $4.7(5)$ \\
\hline$C(322)$ & $0.480(2)$ & $0.116(1)$ & $0.1014(9)$ & $7.9(8)$ \\
\hline$C(323)$ & $0.534(2)$ & $0.152(1)$ & $0.077(1)$ & $10(1)$ \\
\hline C(324) & $0.578(2)$ & $0.129(1)$ & $0.048(1)$ & $8.4(9)$ \\
\hline$C(325)$ & $0.570(2)$ & $0.068(1)$ & $0.040(1)$ & $14(1)$ \\
\hline$C(326)$ & $0.510(2)$ & $0.032(1)$ & $0.064(1)$ & $14(1)$ \\
\hline C(411) & $0.562(1)$ & $0.0637(7)$ & $0.4222(5)$ & $2.7(4)$ \\
\hline$C(412)$ & $0.496(1)$ & $0.0533(7)$ & $0.4505(5)$ & $3.6(4)$ \\
\hline$C(413)$ & $0.507(1)$ & $0.0809(8)$ & $0.4949(6)$ & $3.9(5)$ \\
\hline$C(414)$ & $0.582(2)$ & $0.118(1)$ & $0.5072(7)$ & $6.4(7)$ \\
\hline$C(415)$ & $0.651(2)$ & $0.130(1)$ & $0.4788(7)$ & $7.7(7)$ \\
\hline$C(416)$ & $0.638(2)$ & $0.1021(10)$ & $0.4355(6)$ & $6.0(6)$ \\
\hline$C(421)$ & $0.507(1)$ & $-0.0434(6)$ & $0.3690(5)$ & $2.6(4)$ \\
\hline$C(422)$ & $0.424(1)$ & $-0.0666(8)$ & $0.3455(6)$ & $3.6(4)$ \\
\hline$C(423)$ & $0.404(1)$ & $-0.1273(8)$ & $0.3479(6)$ & $3.8(5)$ \\
\hline$C(424)$ & $0.468(1)$ & $-0.1655(7)$ & $0.3743(6)$ & $4.1(5)$ \\
\hline$C(425)$ & $0.553(1)$ & $-0.1408(8)$ & $0.3975(6)$ & $3.9(5)$ \\
\hline$C(426)$ & $0.573(1)$ & $-0.0804(8)$ & $0.3955(6)$ & $3.7(5)$ \\
\hline$C(511)$ & $0.711(1)$ & $0.0510(8)$ & $0.2536(6)$ & $3.8(5)$ \\
\hline C(512) & $0.686(1)$ & $0.0689(9)$ & $0.2081(8)$ & $5.3(6)$ \\
\hline$C(513)$ & $0.753(2)$ & $0.091(1)$ & $0.1844(9)$ & $8.2(8)$ \\
\hline$C(514)$ & $0.846(2)$ & $0.099(1)$ & $0.207(1)$ & $8.2(9)$ \\
\hline C(515) & $0.873(1)$ & $0.0832(10)$ & $0.2515(10)$ & $6.6(7)$ \\
\hline$C(516)$ & $0.803(1)$ & $0.0596(8)$ & $0.2772(6)$ & $4.5(5)$ \\
\hline$C(611)$ & $0.716(1)$ & $-0.1288(7)$ & $0.2100(5)$ & $2.4(4)$ \\
\hline C(612) & $0.7102(10)$ & $-0.1813(7)$ & $0.1836(5)$ & $2.9(4)$ \\
\hline$C(613)$ & $0.781(1)$ & $-0.1965(8)$ & $0.1588(6)$ & $4.0(5)$ \\
\hline$C(614)$ & $0.860(1)$ & $-0.1600(9)$ & $0.1608(6)$ & $4.2(5)$ \\
\hline C(615) & $0.868(1)$ & $-0.1089(8)$ & $0.1879(6)$ & $4.4(5)$ \\
\hline$C(616)$ & $0.795(1)$ & $-0.0922(7)$ & $0.2102(6)$ & $3.4(4)$ \\
\hline$C(621)$ & $0.5687(10)$ & $-0.1739(7)$ & $0.2558(5)$ & $2.3(4)$ \\
\hline$C(622)$ & $0.477(1)$ & $-0.1949(7)$ & $0.2392(5)$ & $3.0(4)$ \\
\hline$C(623)$ & $0.443(1)$ & $-0.2472(8)$ & $0.2562(5)$ & $3.5(4)$ \\
\hline$C(624)$ & $0.501(1)$ & $-0.2800(7)$ & $0.2886(6)$ & $4.1(5)$ \\
\hline C(625) & $0.595(1)$ & $-0.2619(7)$ & $0.3047(5)$ & $3.8(5)$ \\
\hline$C(626)$ & $0.628(1)$ & $-0.2083(7)$ & $0.2881(5)$ & $3.0(4)$ \\
\hline$C(711)$ & $0.908(2)$ & $0.075(1)$ & $0.423(1)$ & 10(1) \\
\hline C(712) & $0.889(3)$ & $0.052(2)$ & $0.468(1)$ & $15(1)$ \\
\hline C(713) & $0.998(2)$ & $0.056(1)$ & $0.402(2)$ & $18(1)$ \\
\hline$C(721)$ & $0.641(3)$ & $0.527(1)$ & $0.330(2)$ & $15(1)$ \\
\hline$C(722)$ & $0.567(3)$ & $0.555(2)$ & $0.293(2)$ & $15(1)$ \\
\hline$C(723)$ & $0.687(2)$ & $0.551(2)$ & $0.380(2)$ & 19(1) \\
\hline
\end{tabular}




\begin{tabular}{|c|c|c|c|c|}
\hline $\mathrm{H}(1)$ & 0.1962 & 0.0612 & 0.2522 & 4.0 \\
\hline $\mathrm{H}(2)$ & 0.1908 & 0.0988 & 0.2073 & 4.0 \\
\hline $\mathrm{H}(3)$ & 0.2626 & 0.0586 & 0.1339 & 3.0 \\
\hline $\mathrm{H}(4)$ & 0.3488 & 0.0983 & 0.1560 & 3.0 \\
\hline $\mathrm{H}(5)$ & 0.6758 & 0.0811 & 0.3436 & 2.1 \\
\hline $\mathrm{H}(6)$ & 0.6973 & 0.0148 & 0.3597 & 2.1 \\
\hline $\mathrm{H}(7)$ & 0.7272 & -0.0669 & 0.2949 & 2.5 \\
\hline $\mathrm{H}(8)$ & 0.6338 & -0.0823 & 0.3139 & 2.5 \\
\hline $\mathrm{H}(9)$ & 0.1855 & 0.1225 & 0.4597 & 5.5 \\
\hline $\mathrm{H}(10)$ & 0.2049 & 0.1400 & 0.4109 & 5.5 \\
\hline $\mathrm{H}(11)$ & 0.2743 & 0.0963 & 0.4417 & 5.5 \\
\hline $\mathrm{H}(12)$ & 0.3609 & 0.3187 & 0.5767 & 5.7 \\
\hline $\mathrm{H}(13)$ & 0.2526 & 0.3029 & 0.5635 & 5.7 \\
\hline $\mathrm{H}(14)$ & 0.3229 & 0.2569 & 0.5912 & 5.7 \\
\hline $\mathrm{H}(15)$ & 0.5702 & 0.2647 & 0.4536 & 5.7 \\
\hline $\mathrm{H}(16)$ & 0.5095 & 0.2494 & 0.4059 & 5.7 \\
\hline $\mathrm{H}(17)$ & 0.5041 & 0.3136 & 0.4268 & 5.7 \\
\hline $\mathrm{H}(18)$ & 0.7958 & -0.0767 & 0.0843 & 5.6 \\
\hline $\mathrm{H}(19)$ & 0.7287 & -0.0418 & 0.0458 & 5.6 \\
\hline $\mathrm{H}(20)$ & 0.6957 & -0.0546 & 0.0926 & 5.6 \\
\hline $\mathrm{H}(21)$ & 0.7440 & -0.2893 & -0.0254 & 9.8 \\
\hline $\mathrm{H}(22)$ & 0.6795 & -0.2515 & -0.0630 & 9.8 \\
\hline $\mathrm{H}(23)$ & 0.7818 & -0.2289 & -0.0425 & 9.8 \\
\hline $\mathrm{H}(24)$ & 0.4232 & -0.2069 & 0.0623 & 5.4 \\
\hline $\mathrm{H}(25)$ & 0.4555 & -0.2723 & 0.0529 & 5.4 \\
\hline $\mathrm{H}(26)$ & 0.4902 & -0.2422 & 0.1005 & 5.4 \\
\hline $\mathrm{H}(27)$ & 0.2272 & 0.1986 & 0.5189 & 4.9 \\
\hline $\mathrm{H}(28)$ & 0.4570 & 0.3065 & 0.5130 & 4.9 \\
\hline $\mathrm{H}(29)$ & 0.7807 & -0.1462 & 0.0050 & 5.9 \\
\hline $\mathrm{H}(30)$ & 0.5759 & -0.2757 & 0.0003 & 6.1 \\
\hline $\mathrm{H}(31)$ & 0.1128 & 0.1316 & 0.2832 & 5.0 \\
\hline $\mathrm{H}(32)$ & 0.0191 & 0.2056 & 0.3199 & 5.8 \\
\hline $\mathrm{H}(33)$ & 0.0826 & 0.2978 & 0.3430 & 4.9 \\
\hline $\mathrm{H}(34)$ & 0.2361 & 0.3251 & 0.3365 & 6.0 \\
\hline $\mathrm{H}(35)$ & 0.3301 & 0.2568 & 0.2962 & 5.0 \\
\hline $\mathrm{H}(36)$ & 0.4442 & 0.0257 & 0.4396 & 3.9 \\
\hline $\mathrm{H}(37)$ & 0.4580 & 0.0732 & 0.5151 & 4.2 \\
\hline $\mathrm{H}(38)$ & 0.5868 & 0.1368 & 0.5373 & 6.1 \\
\hline $\mathrm{H}(39)$ & 0.7037 & 0.1565 & 0.4918 & 7.9 \\
\hline $\mathrm{H}(40)$ & 0.6876 & 0.1079 & 0.4141 & 6.5 \\
\hline $\mathrm{H}(41)$ & 0.2798 & 0.0385 & 0.0452 & 4.5 \\
\hline $\mathrm{H}(42)$ & 0.1619 & -0.0174 & -0.0033 & 5.4 \\
\hline $\mathrm{H}(43)$ & 0.1378 & -0.1210 & 0.0063 & 5.0 \\
\hline $\mathrm{H}(44)$ & 0.2335 & -0.1738 & 0.0700 & 5.2 \\
\hline $\mathrm{H}(45)$ & 0.3463 & -0.1184 & 0.1210 & 3.6 \\
\hline $\mathrm{H}(46)$ & 0.4428 & 0.1340 & 0.1233 & 8.0 \\
\hline $\mathrm{H}(47)$ & 0.5453 & 0.1966 & 0.0831 & 12.1 \\
\hline $\mathrm{H}(48)$ & 0.6251 & 0.1522 & 0.0336 & 8.9 \\
\hline $\mathrm{H}(49)$ & 0.5978 & 0.0510 & 0.0114 & 10.9 \\
\hline $\mathrm{H}(50)$ & 0.4921 & -0.0128 & 0.0527 & 11.0 \\
\hline $\mathrm{H}(51)$ & 0.3780 & -0.0388 & 0.3269 & 3.8 \\
\hline $\mathrm{H}(52)$ & 0.3435 & -0.1447 & 0.3311 & 4.1 \\
\hline $\mathrm{H}(53)$ & 0.4560 & -0.2088 & 0.3759 & 4.0 \\
\hline $\mathrm{H}(54)$ & 0.5980 & -0.1657 & 0.4182 & 4.1 \\
\hline $\mathrm{H}(55)$ & 0.6337 & -0.0630 & 0.4102 & 3.7 \\
\hline $\mathrm{H}(56)$ & 0.4337 & -0.1733 & 0.2147 & 3.2 \\
\hline $\mathrm{H}(57)$ & 0.3768 & -0.2625 & 0.2482 & 3.9 \\
\hline $\mathrm{H}(58)$ & 0.4815 & -0.3180 & 0.3005 & 3.8 \\
\hline
\end{tabular}




$\begin{array}{llrll}\mathrm{H}(59) & 0.6356 & -0.2858 & 0.3286 & 3.5 \\ \mathrm{H}(60) & 0.6933 & -0.1939 & 0.3000 & 3.1 \\ \mathrm{H}(61) & 0.2220 & 0.2087 & 0.1861 & 4.7 \\ \mathrm{H}(62) & 0.2857 & 0.2786 & 0.1355 & 7.9 \\ \mathrm{H}(63) & 0.4489 & 0.2822 & 0.1380 & 7.7 \\ \mathrm{H}(64) & 0.5589 & 0.2375 & 0.1924 & 8.7 \\ \mathrm{H}(65) & 0.4998 & 0.1677 & 0.2496 & 4.9 \\ \mathrm{H}(66) & 0.1356 & -0.0052 & 0.1493 & 3.0 \\ \mathrm{H}(67) & 0.0515 & -0.0980 & 0.1326 & 4.0 \\ \mathrm{H}(68) & 0.0887 & -0.1817 & 0.1801 & 4.6 \\ \mathrm{H}(69) & 0.2356 & -0.1824 & 0.2341 & 4.9 \\ \mathrm{H}(70) & 0.3291 & -0.0941 & 0.2473 & 3.5 \\ \mathrm{H}(71) & 0.6174 & 0.0644 & 0.1942 & 5.4 \\ \mathrm{H}(72) & 0.7335 & 0.1027 & 0.1502 & 7.2 \\ \mathrm{H}(73) & 0.8978 & 0.1145 & 0.1901 & 6.8 \\ \mathrm{H}(74) & 0.9368 & 0.0910 & 0.2665 & 4.7 \\ \mathrm{H}(75) & 0.8196 & 0.0485 & 0.3112 & 2.6 \\ \mathrm{H}(76) & 0.6554 & -0.2096 & 0.1831 & 4.7 \\ \mathrm{H}(77) & 0.7757 & -0.2317 & 0.1392 & 4.2 \\ \mathrm{H}(78) & 0.9102 & -0.1705 & 0.1434 & 4.3 \\ \mathrm{H}(79) & 0.9267 & -0.0834 & 0.1886 & 3.3 \\ \mathrm{H}(80) & 0.7985 & -0.0550 & 0.2279 & \end{array}$

a Estimated standard deviations are given in parentheses. ${ }^{b}$ All non-hydrogen atoms were assigned with anisotropic thermal parameters given as the isotropic equivalent displacement parameter defined as $B_{\text {eq }}=\left(8 \pi^{2} / 3\right)\left(U_{11}\left(a a^{*}\right)^{2}+U_{22}\left(b b^{*}\right)^{2}+U_{33}\left(c c^{*}\right)^{2}+2 U_{12} a a^{*} b b^{*} \cos \gamma+2 U_{13} a a^{*} c c^{*} \cos \beta+\right.$ $\left.2 U_{23} b b^{*} c c^{*} \cos \alpha\right)$. ${ }^{\mathrm{C}}$ The positions of all hydrogen atoms except for solvent molecules were calculated with $\mathrm{C}-\mathrm{H}=0.95 \AA$ and were not refined with the appropriate $B$ iso.

Table S1-3. Final Anisotropic Thermal Parameters for 2·2( $\left.\mathrm{CH}_{3}\right)_{2} \mathrm{CO}^{\mathrm{a}, \mathrm{b}}$

\begin{tabular}{lllllcr}
\hline atom & $U_{11}$ & $U_{22}$ & $U_{33}$ & $U_{12}$ & $U_{13}$ & $U_{23}$ \\
\hline $\mathrm{Pt}(1)$ & $0.0297(4)$ & $0.0261(4)$ & $0.0321(4)$ & $0.0021(3)$ & $0.0079(3)$ & $-0.0033(3)$ \\
$\mathrm{Pt}(2)$ & $0.0262(3)$ & $0.0247(4)$ & $0.0303(4)$ & $0.0016(3)$ & $0.0068(3)$ & $-0.0014(3)$ \\
$\mathrm{Pt}(3)$ & $0.0293(4)$ & $0.0261(4)$ & $0.0285(4)$ & $0.0041(3)$ & $0.0077(3)$ & $0.0012(3)$ \\
$\mathrm{P}(1)$ & $0.033(2)$ & $0.028(2)$ & $0.035(3)$ & $0.006(2)$ & $0.010(2)$ & $-0.001(2)$ \\
$\mathrm{P}(2)$ & $0.030(2)$ & $0.034(2)$ & $0.026(2)$ & $0.003(2)$ & $0.010(2)$ & $0.001(2)$ \\
$\mathrm{P}(3)$ & $0.036(2)$ & $0.035(3)$ & $0.030(2)$ & $0.012(2)$ & $0.010(2)$ & $0.007(2)$ \\
$\mathrm{P}(4)$ & $0.038(2)$ & $0.030(3)$ & $0.033(2)$ & $0.002(2)$ & $0.005(2)$ & $-0.005(2)$ \\
$\mathrm{P}(5)$ & $0.025(2)$ & $0.026(2)$ & $0.042(3)$ & $-0.001(2)$ & $0.004(2)$ & $-0.003(2)$ \\
$\mathrm{P}(6)$ & $0.024(2)$ & $0.024(2)$ & $0.032(2)$ & $0.001(2)$ & $0.004(2)$ & $0.000(2)$ \\
$\mathrm{P}(11)$ & $0.041(3)$ & $0.063(4)$ & $0.058(4)$ & $0.006(3)$ & $0.004(2)$ & $0.003(3)$ \\
$\mathrm{P}(12)$ & $0.077(4)$ & $0.080(5)$ & $0.055(4)$ & $0.036(3)$ & $-0.012(3)$ & $-0.018(3)$ \\
$\mathrm{F}(11)$ & $0.12(1)$ & $0.072(10)$ & $0.20(2)$ & $0.045(9)$ & $-0.07(1)$ & $-0.03(1)$ \\
$\mathrm{F}(12)$ & $0.22(2)$ & $0.21(2)$ & $0.056(9)$ & $-0.01(2)$ & $0.03(1)$ & $-0.01(1)$ \\
$\mathrm{F}(13)$ & $0.076(9)$ & $0.15(1)$ & $0.18(2)$ & $-0.046(9)$ & $-0.021(9)$ & $0.04(1)$ \\
$\mathrm{F}(14)$ & $0.051(9)$ & $0.19(2)$ & $0.38(3)$ & $-0.01(1)$ & $0.00(1)$ & $0.14(2)$ \\
$\mathrm{F}(15)$ & $0.21(2)$ & $0.33(3)$ & $0.10(1)$ & $0.17(2)$ & $0.06(1)$ & $0.03(2)$ \\
$\mathrm{F}(16)$ & $0.21(2)$ & $0.10(1)$ & $0.22(2)$ & $0.10(1)$ & $-0.10(1)$ & $-0.06(1)$ \\
$\mathrm{F}(21)$ & $0.13(1)$ & $0.063(9)$ & $0.21(2)$ & $0.010(8)$ & $0.07(1)$ & $-0.02(1)$ \\
$\mathrm{F}(22)$ & $0.070(8)$ & $0.15(1)$ & $0.083(9)$ & $0.008(8)$ & $-0.021(7)$ & $-0.024(9)$ \\
$\mathrm{F}(23)$ & $0.11(1)$ & $0.18(2)$ & $0.063(9)$ & $0.04(1)$ & $-0.006(8)$ & $0.008(9)$ \\
$\mathrm{F}(24)$ & $0.067(8)$ & $0.14(1)$ & $0.101(10)$ & $0.052(8)$ & $0.011(7)$ & $0.024(9)$ \\
$\mathrm{F}(25)$ & $0.24(2)$ & $0.07(1)$ & $0.18(2)$ & $0.03(1)$ & $0.09(1)$ & $-0.04(1)$ \\
$\mathrm{F}(26)$ & $0.099(10)$ & $0.21(2)$ & $0.043(7)$ & $0.04(1)$ & $-0.007(7)$ & $-0.005(9)$ \\
$\mathrm{O}(701)$ & $0.049(9)$ & $0.12(1)$ & $0.13(1)$ & $0.014(9)$ & $-0.027(9)$ & $-0.02(1)$
\end{tabular}




\begin{tabular}{|c|c|c|c|c|c|c|}
\hline $\mathrm{O}(702)$ & $0.18(2)$ & $0.16(2)$ & $0.10(1)$ & $0.02(2)$ & $0.06(1)$ & $-0.04(1)$ \\
\hline $\mathrm{N}(1)$ & $0.049(9)$ & $0.038(9)$ & $0.040(9)$ & $0.009(7)$ & $0.001(7)$ & $-0.010(8)$ \\
\hline $\mathrm{N}(2)$ & $0.055(9)$ & $0.050(9)$ & $0.031(8)$ & $0.017(8)$ & $0.018(7)$ & $-0.002(8)$ \\
\hline$C(1)$ & $0.05(1)$ & $0.021(9)$ & $0.05(1)$ & $0.009(8)$ & $0.029(9)$ & $0.003(9)$ \\
\hline$C(2)$ & $0.031(9)$ & $0.04(1)$ & $0.023(9)$ & $0.021(8)$ & $0.008(7)$ & $0.007(8)$ \\
\hline$C(3)$ & $0.029(9)$ & $0.04(1)$ & $0.04(1)$ & $0.007(8)$ & $0.005(8)$ & $-0.015(8)$ \\
\hline$C(4)$ & $0.043(10)$ & $0.027(9)$ & $0.04(1)$ & $0.004(8)$ & $0.017(8)$ & $-0.002(8)$ \\
\hline$C(5)$ & $0.024(8)$ & $0.035(10)$ & $0.05(1)$ & $0.005(7)$ & $-0.008(7)$ & $0.004(8)$ \\
\hline$C(6)$ & $0.027(8)$ & $0.020(9)$ & $0.043(10)$ & $0.011(7)$ & $0.010(7)$ & $0.007(7)$ \\
\hline$C(11)$ & $0.038(10)$ & $0.04(1)$ & $0.018(9)$ & $0.025(8)$ & $0.005(8)$ & $-0.013(8)$ \\
\hline$C(12)$ & $0.05(1)$ & $0.07(1)$ & $0.03(1)$ & $0.016(10)$ & $-0.004(9)$ & $-0.026(10)$ \\
\hline$C(13)$ & $0.04(1)$ & $0.07(1)$ & $0.06(1)$ & $0.017(10)$ & $0.006(10)$ & $0.00(1)$ \\
\hline$C(14)$ & $0.07(1)$ & $0.05(1)$ & $0.04(1)$ & $0.02(1)$ & $0.01(1)$ & $0.002(10)$ \\
\hline$C(15)$ & $0.09(2)$ & $0.05(1)$ & $0.04(1)$ & $-0.01(1)$ & $0.00(1)$ & $0.00(1)$ \\
\hline$C(16)$ & $0.06(1)$ & $0.05(1)$ & $0.05(1)$ & $0.005(10)$ & $0.02(1)$ & $-0.02(1)$ \\
\hline$C(17)$ & $0.05(1)$ & $0.11(2)$ & $0.09(2)$ & $-0.03(1)$ & $0.03(1)$ & $-0.06(1)$ \\
\hline$C(18)$ & $0.11(2)$ & $0.08(1)$ & $0.04(1)$ & $0.06(1)$ & $0.00(1)$ & $-0.01(1)$ \\
\hline$C(19)$ & $0.09(2)$ & $0.07(2)$ & $0.09(2)$ & $-0.03(1)$ & $0.04(1)$ & $-0.02(1)$ \\
\hline$C(21)$ & $0.06(1)$ & $0.04(1)$ & $0.03(1)$ & $0.009(10)$ & $0.016(9)$ & $0.009(9)$ \\
\hline$C(22)$ & $0.05(1)$ & $0.05(1)$ & $0.014(9)$ & $0.026(10)$ & $-0.010(8)$ & $0.006(9)$ \\
\hline$C(23)$ & $0.08(2)$ & $0.07(2)$ & $0.09(2)$ & $0.02(1)$ & $0.03(1)$ & $-0.02(1)$ \\
\hline$C(24)$ & $0.11(2)$ & $0.09(2)$ & $0.05(1)$ & $0.05(2)$ & $0.04(1)$ & $0.01(1)$ \\
\hline$C(25)$ & $0.11(2)$ & $0.07(1)$ & $0.04(1)$ & $0.02(1)$ & $0.01(1)$ & $-0.02(1)$ \\
\hline$C(26)$ & $0.06(1)$ & $0.05(1)$ & $0.05(1)$ & $0.006(10)$ & $-0.001(9)$ & $-0.024(10)$ \\
\hline$C(27)$ & $0.07(1)$ & $0.07(1)$ & $0.10(2)$ & $0.00(1)$ & $0.04(1)$ & $0.03(1)$ \\
\hline$C(28)$ & $0.19(3)$ & $0.13(2)$ & $0.09(2)$ & $0.07(2)$ & $0.06(2)$ & $-0.01(2)$ \\
\hline$C(29)$ & $0.06(1)$ & $0.08(2)$ & $0.10(2)$ & $-0.01(1)$ & $0.02(1)$ & $-0.01(1)$ \\
\hline$C(111)$ & $0.021(9)$ & $0.04(1)$ & $0.05(1)$ & $0.011(8)$ & $-0.004(8)$ & $0.014(9)$ \\
\hline$C(112)$ & $0.05(1)$ & $0.06(1)$ & $0.08(1)$ & $0.019(10)$ & $0.01(1)$ & $-0.02(1)$ \\
\hline$C(113)$ & $0.06(1)$ & $0.05(1)$ & $0.10(2)$ & $0.02(1)$ & $0.02(1)$ & $-0.01(1)$ \\
\hline$C(114)$ & $0.06(1)$ & $0.05(1)$ & $0.07(1)$ & $0.04(1)$ & $0.03(1)$ & $0.00(1)$ \\
\hline$C(115)$ & $0.10(2)$ & $0.05(1)$ & $0.08(2)$ & $0.04(1)$ & $0.03(1)$ & $-0.01(1)$ \\
\hline$C(116)$ & $0.04(1)$ & $0.05(1)$ & $0.09(2)$ & $-0.008(10)$ & $0.02(1)$ & $-0.01(1)$ \\
\hline$C(121)$ & $0.07(1)$ & $0.018(9)$ & $0.04(1)$ & $-0.016(9)$ & $0.028(10)$ & $-0.027(8)$ \\
\hline$C(122)$ & $0.12(2)$ & $0.05(1)$ & $0.04(1)$ & $0.02(1)$ & $0.01(1)$ & $0.02(1)$ \\
\hline$C(123)$ & $0.17(3)$ & $0.04(1)$ & $0.06(2)$ & $0.02(2)$ & $0.04(2)$ & $0.03(1)$ \\
\hline$C(124)$ & $0.22(4)$ & $0.06(2)$ & $0.11(3)$ & $-0.05(2)$ & $0.09(3)$ & $0.00(2)$ \\
\hline$C(125)$ & $0.16(3)$ & $0.06(2)$ & $0.12(2)$ & $-0.06(2)$ & $0.08(2)$ & $-0.02(2)$ \\
\hline$C(126)$ & $0.08(1)$ & $0.05(1)$ & $0.05(1)$ & $-0.04(1)$ & $0.02(1)$ & $-0.01(1)$ \\
\hline$C(211)$ & $0.024(8)$ & $0.015(9)$ & $0.05(1)$ & $0.009(7)$ & $0.021(8)$ & $-0.008(8)$ \\
\hline$C(212)$ & $0.028(9)$ & $0.04(1)$ & $0.035(10)$ & $0.006(8)$ & $-0.011(8)$ & $0.001(8)$ \\
\hline$C(213)$ & $0.025(9)$ & $0.07(1)$ & $0.05(1)$ & $-0.007(10)$ & $-0.003(8)$ & $-0.03(1)$ \\
\hline$C(214)$ & $0.04(1)$ & $0.04(1)$ & $0.10(2)$ & $0.009(9)$ & $0.02(1)$ & $0.03(1)$ \\
\hline$C(215)$ & $0.04(1)$ & $0.04(1)$ & $0.10(2)$ & $0.010(10)$ & $0.01(1)$ & $0.02(1)$ \\
\hline$C(216)$ & $0.030(9)$ & $0.04(1)$ & $0.05(1)$ & $-0.007(8)$ & $0.007(8)$ & $-0.016(9)$ \\
\hline$C(311)$ & $0.05(1)$ & $0.05(1)$ & $0.008(8)$ & $0.022(9)$ & $0.002(7)$ & $0.000(8)$ \\
\hline$C(312)$ & $0.07(1)$ & $0.07(1)$ & $0.017(9)$ & $0.03(1)$ & $-0.006(9)$ & $0.005(9)$ \\
\hline$C(313)$ & $0.08(2)$ & $0.09(2)$ & $0.04(1)$ & $0.05(1)$ & $-0.01(1)$ & $0.00(1)$ \\
\hline$C(314)$ & $0.04(1)$ & $0.10(2)$ & $0.04(1)$ & $0.00(1)$ & $-0.001(9)$ & $-0.02(1)$ \\
\hline$C(315)$ & $0.04(1)$ & $0.08(1)$ & $0.05(1)$ & $-0.01(1)$ & $-0.004(9)$ & $-0.01(1)$ \\
\hline$C(316)$ & $0.04(1)$ & $0.08(2)$ & $0.03(1)$ & $-0.01(1)$ & $0.006(9)$ & $0.00(1)$ \\
\hline$C(321)$ & $0.04(1)$ & $0.08(2)$ & $0.06(1)$ & $0.02(1)$ & $0.030(10)$ & $0.03(1)$ \\
\hline$C(322)$ & $0.12(2)$ & $0.06(2)$ & $0.14(2)$ & $-0.02(1)$ & $0.07(2)$ & $0.02(2)$ \\
\hline$C(323)$ & $0.15(3)$ & $0.11(2)$ & $0.15(3)$ & $-0.04(2)$ & $0.07(2)$ & $0.03(2)$ \\
\hline$C(324)$ & $0.08(2)$ & $0.10(2)$ & $0.15(3)$ & $0.03(2)$ & $0.05(2)$ & $0.09(2)$ \\
\hline$C(325)$ & $0.23(3)$ & $0.11(2)$ & $0.28(4)$ & $0.11(2)$ & $0.23(3)$ & $0.14(2)$ \\
\hline$C(326)$ & $0.25(4)$ & $0.08(2)$ & $0.29(4)$ & $0.12(2)$ & $0.24(3)$ & $0.13(2)$ \\
\hline$C(411)$ & $0.035(9)$ & $0.04(1)$ & $0.030(10)$ & $0.000(8)$ & $0.004(8)$ & $-0.002(8)$ \\
\hline
\end{tabular}




\begin{tabular}{|c|c|c|c|c|c|c|}
\hline$C(412)$ & $0.08(1)$ & $0.023(10)$ & $0.04(1)$ & $0.014(9)$ & $0.002(9)$ & $0.001(8)$ \\
\hline$C(413)$ & $0.08(1)$ & $0.04(1)$ & $0.03(1)$ & $0.01(1)$ & $0.016(10)$ & $-0.007(9)$ \\
\hline$C(414)$ & $0.12(2)$ & $0.07(2)$ & $0.05(1)$ & $-0.01(1)$ & $0.02(1)$ & $-0.01(1)$ \\
\hline$C(415)$ & $0.11(2)$ & $0.13(2)$ & $0.05(2)$ & $-0.05(2)$ & $0.02(1)$ & $-0.02(1)$ \\
\hline$C(416)$ & $0.09(2)$ & $0.10(2)$ & $0.03(1)$ & $-0.03(1)$ & $0.00(1)$ & $-0.02(1)$ \\
\hline$C(421)$ & $0.05(1)$ & $0.020(9)$ & $0.037(10)$ & $-0.001(8)$ & $0.021(9)$ & $-0.006(8)$ \\
\hline$C(422)$ & $0.05(1)$ & $0.04(1)$ & $0.05(1)$ & $0.000(9)$ & $0.004(9)$ & $0.001(9)$ \\
\hline$C(423)$ & $0.06(1)$ & $0.04(1)$ & $0.05(1)$ & $-0.020(10)$ & $0.015(9)$ & $0.000(10)$ \\
\hline$C(424)$ & $0.08(1)$ & $0.02(1)$ & $0.06(1)$ & $0.00(1)$ & $0.02(1)$ & $0.007(9)$ \\
\hline$C(425)$ & $0.06(1)$ & $0.05(1)$ & $0.05(1)$ & $0.02(1)$ & $0.013(10)$ & $-0.005(10)$ \\
\hline$C(426)$ & $0.04(1)$ & $0.04(1)$ & $0.05(1)$ & $0.000(9)$ & $0.004(9)$ & $-0.008(10)$ \\
\hline$C(511)$ & $0.03(1)$ & $0.04(1)$ & $0.07(1)$ & $-0.002(8)$ & $0.013(10)$ & $-0.02(1)$ \\
\hline$C(512)$ & $0.03(1)$ & $0.06(1)$ & $0.11(2)$ & $0.005(9)$ & $0.03(1)$ & $0.03(1)$ \\
\hline$C(513)$ & $0.07(2)$ & $0.12(2)$ & $0.12(2)$ & $0.01(2)$ & $0.04(2)$ & $0.07(2)$ \\
\hline$C(514)$ & $0.06(2)$ & $0.08(2)$ & $0.18(3)$ & $0.00(1)$ & $0.04(2)$ & $0.04(2)$ \\
\hline$C(515)$ & $0.04(1)$ & $0.06(2)$ & $0.14(2)$ & $-0.01(1)$ & $0.00(1)$ & $0.01(2)$ \\
\hline$C(516)$ & $0.03(1)$ & $0.06(1)$ & $0.08(1)$ & $-0.019(9)$ & $0.010(10)$ & $-0.01(1)$ \\
\hline$C(611)$ & $0.038(9)$ & $0.022(9)$ & $0.032(9)$ & $0.008(8)$ & $0.008(7)$ & $-0.004(8)$ \\
\hline$C(612)$ & $0.025(9)$ & $0.05(1)$ & $0.036(10)$ & $0.008(8)$ & $0.005(8)$ & $0.006(9)$ \\
\hline$C(613)$ & $0.04(1)$ & $0.06(1)$ & $0.05(1)$ & $0.018(10)$ & $0.004(9)$ & $-0.014(10)$ \\
\hline$C(614)$ & $0.04(1)$ & $0.07(1)$ & $0.05(1)$ & $0.02(1)$ & $0.017(9)$ & $-0.01(1)$ \\
\hline$C(615)$ & $0.04(1)$ & $0.06(1)$ & $0.07(1)$ & $-0.013(9)$ & $0.03(1)$ & $-0.02(1)$ \\
\hline$C(616)$ & $0.05(1)$ & $0.030(10)$ & $0.06(1)$ & $-0.003(9)$ & $0.022(9)$ & $-0.017(9)$ \\
\hline$C(621)$ & $0.024(8)$ & $0.034(10)$ & $0.031(9)$ & $0.005(7)$ & $0.004(7)$ & $-0.009(8)$ \\
\hline$C(622)$ & $0.05(1)$ & $0.025(9)$ & $0.04(1)$ & $0.001(8)$ & $0.021(9)$ & $0.015(8)$ \\
\hline$C(623)$ & $0.06(1)$ & $0.04(1)$ & $0.03(1)$ & $-0.024(9)$ & $0.005(9)$ & $-0.007(9)$ \\
\hline$C(624)$ & $0.09(2)$ & $0.03(1)$ & $0.05(1)$ & $-0.01(1)$ & $0.03(1)$ & $0.012(9)$ \\
\hline$C(625)$ & $0.09(1)$ & $0.016(10)$ & $0.03(1)$ & $0.009(9)$ & $0.00(1)$ & $0.002(8)$ \\
\hline$C(626)$ & $0.038(10)$ & $0.04(1)$ & $0.04(1)$ & $0.006(8)$ & $-0.001(8)$ & $0.000(8)$ \\
\hline$C(711)$ & $0.05(2)$ & $0.08(2)$ & $0.25(4)$ & $0.03(1)$ & $-0.05(2)$ & $-0.06(2)$ \\
\hline$C(712)$ & $0.29(5)$ & $0.15(3)$ & $0.11(3)$ & $0.08(3)$ & $-0.09(3)$ & $-0.03(3)$ \\
\hline$C(713)$ & $0.06(2)$ & $0.10(2)$ & $0.55(8)$ & $-0.04(2)$ & $0.04(3)$ & $-0.09(4)$ \\
\hline$C(721)$ & $0.25(4)$ & $0.07(2)$ & $0.37(6)$ & $-0.07(2)$ & $0.28(5)$ & $-0.13(3)$ \\
\hline$C(722)$ & $0.21(4)$ & $0.11(3)$ & $0.30(6)$ & $0.09(3)$ & $0.17(4)$ & $0.08(3)$ \\
\hline$C(723)$ & $0.15(3)$ & $0.18(3)$ & $0.47(7)$ & $-0.13(3)$ & $0.21(4)$ & $-0.23(4)$ \\
\hline
\end{tabular}

a Estmated standard deviations are given in parentheses. $\mathrm{b}$ Parameters have the form $\exp (-$ $\left.2 \pi^{2}\left(U_{11} h^{2} a * 2+\cdots+2 U_{12} h k a^{*} b^{*} \cos \gamma+\cdots\right)\right)$.

Table S1-4. Bond Lengths for Non-hydrogen Atoms of 2.2(CH3) $)_{2} \mathrm{CO}^{\mathrm{a}}$

\begin{tabular}{llllll}
\hline atom & atom & distance & atom & atom & distance \\
\hline $\operatorname{Pt}(1)$ & $\mathrm{Pt}(2)$ & $2.7134(9)$ & $\mathrm{P}(6)$ & $\mathrm{C}(611)$ & $1.83(1)$ \\
$\operatorname{Pt}(1)$ & $\mathrm{P}(1)$ & $2.276(4)$ & $\mathrm{P}(6)$ & $\mathrm{C}(621)$ & $1.81(2)$ \\
$\operatorname{Pt}(1)$ & $\mathrm{P}(4)$ & $2.267(4)$ & $\mathrm{P}(11)$ & $\mathrm{F}(11)$ & $1.57(1)$ \\
$\operatorname{Pt}(1)$ & $\mathrm{C}(1)$ & $1.97(2)$ & $\mathrm{P}(11)$ & $\mathrm{F}(12)$ & $1.49(1)$ \\
$\operatorname{Pt}(2)$ & $\mathrm{Pt}(3)$ & $2.7045(8)$ & $\mathrm{P}(11)$ & $\mathrm{F}(13)$ & $1.52(1)$ \\
$\operatorname{Pt}(2)$ & $\mathrm{P}(2)$ & $2.251(4)$ & $\mathrm{P}(11)$ & $\mathrm{F}(14)$ & $1.51(1)$ \\
$\operatorname{Pt}(2)$ & $\mathrm{P}(5)$ & $2.253(4)$ & $\mathrm{P}(11)$ & $\mathrm{F}(15)$ & $1.56(2)$ \\
$\operatorname{Pt}(3)$ & $\mathrm{P}(3)$ & $2.297(4)$ & $\mathrm{P}(11)$ & $\mathrm{F}(16)$ & $1.52(1)$ \\
$\operatorname{Pt}(3)$ & $\mathrm{P}(6)$ & $2.297(4)$ & $\mathrm{P}(12)$ & $\mathrm{F}(21)$ & $1.54(1)$ \\
$\operatorname{Pt}(3)$ & $\mathrm{C}(2)$ & $1.97(2)$ & $\mathrm{P}(12)$ & $\mathrm{F}(22)$ & $1.58(1)$ \\
$\mathrm{P}(1)$ & $\mathrm{C}(3)$ & $1.83(2)$ & $\mathrm{P}(12)$ & $\mathrm{F}(23)$ & $1.57(1)$ \\
$\mathrm{P}(1)$ & $\mathrm{C}(111)$ & $1.82(2)$ & $\mathrm{P}(12)$ & $\mathrm{F}(24)$ & $1.61(1)$ \\
$\mathrm{P}(1)$ & $\mathrm{C}(121)$ & $1.78(2)$ & $\mathrm{P}(12)$ & $\mathrm{F}(25)$ & $1.56(1)$ \\
$\mathrm{P}(2)$ & $\mathrm{C}(3)$ & $1.82(1)$ & $\mathrm{P}(12)$ & $\mathrm{F}(26)$ & $1.55(1)$ \\
$\mathrm{P}(2)$ & $\mathrm{C}(4)$ & $1.81(2)$ & $\mathrm{O}(701)$ & $\mathrm{C}(711)$ & $1.23(3)$ \\
$\mathrm{P}(2)$ & $\mathrm{C}(211)$ & $1.81(1)$ & $\mathrm{O}(702)$ & $\mathrm{C}(721)$ & $1.18(3)$
\end{tabular}




\begin{tabular}{|c|c|c|c|c|c|}
\hline $\mathrm{P}(3)$ & $C(4)$ & $1.81(1)$ & $\mathrm{N}(1)$ & $C(1)$ & $1.17(2)$ \\
\hline $\mathrm{P}(3)$ & $C(311)$ & $1.80(2)$ & $\mathrm{N}(1)$ & $C(11)$ & $1.39(2)$ \\
\hline$P(3)$ & $C(321)$ & $1.84(2)$ & $\mathrm{N}(2)$ & $C(2)$ & $1.16(2)$ \\
\hline $\mathrm{P}(4)$ & $C(5)$ & $1.84(1)$ & $\mathrm{N}(2)$ & $C(21)$ & $1.37(2)$ \\
\hline $\mathrm{P}(4)$ & $C(411)$ & $1.82(2)$ & $C(11)$ & $C(12)$ & $1.41(2)$ \\
\hline $\mathrm{P}(4)$ & $C(421)$ & $1.84(1)$ & $C(11)$ & $C(16)$ & $1.38(2)$ \\
\hline$P(5)$ & C(5) & $1.82(2)$ & $C(12)$ & $C(13)$ & $1.38(2)$ \\
\hline $\mathrm{P}(5)$ & $C(6)$ & $1.84(1)$ & $C(12)$ & $C(17)$ & $1.46(2)$ \\
\hline$P(5)$ & $C(511)$ & $1.82(2)$ & $C(13)$ & $C(14)$ & $1.37(2)$ \\
\hline$P(6)$ & $C(6)$ & $1.82(2)$ & $C(14)$ & $C(15)$ & $1.38(2)$ \\
\hline$C(14)$ & $C(18)$ & $1.52(2)$ & $C(212)$ & $C(213)$ & $1.40(2)$ \\
\hline$C(15)$ & $C(16)$ & $1.40(2)$ & $C(213)$ & $C(214)$ & $1.36(2)$ \\
\hline$C(16)$ & $C(19)$ & $1.50(2)$ & $C(214)$ & $C(215)$ & $1.39(2)$ \\
\hline$C(21)$ & $C(22)$ & $1.41(2)$ & $C(215)$ & $C(216)$ & $1.36(2)$ \\
\hline$C(21)$ & $C(26)$ & $1.39(2)$ & $C(311)$ & $C(312)$ & $1.38(2)$ \\
\hline$C(22)$ & $C(23)$ & $1.39(2)$ & $C(311)$ & $C(316)$ & $1.42(2)$ \\
\hline$C(22)$ & $C(27)$ & $1.48(3)$ & $C(312)$ & $C(313)$ & $1.37(3)$ \\
\hline$C(23)$ & $C(24)$ & $1.34(3)$ & $C(313)$ & $C(314)$ & $1.39(3)$ \\
\hline$C(24)$ & $C(25)$ & $1.39(3)$ & $C(314)$ & $C(315)$ & $1.42(2)$ \\
\hline$C(24)$ & $C(28)$ & $1.56(3)$ & $C(315)$ & $C(316)$ & $1.32(2)$ \\
\hline$C(25)$ & $C(26)$ & $1.40(2)$ & $C(321)$ & $C(322)$ & $1.30(3)$ \\
\hline$C(26)$ & $C(29)$ & $1.50(2)$ & $C(321)$ & $C(326)$ & $1.30(3)$ \\
\hline$C(111)$ & $C(112)$ & $1.37(2)$ & $C(322)$ & $C(323)$ & $1.38(3)$ \\
\hline$C(111)$ & $C(116)$ & $1.37(2)$ & $C(323)$ & $C(324)$ & $1.26(3)$ \\
\hline$C(112)$ & $C(113)$ & $1.44(2)$ & $C(324)$ & $C(325)$ & $1.38(4)$ \\
\hline$C(113)$ & $C(114)$ & $1.36(2)$ & $C(325)$ & $C(326)$ & $1.42(3)$ \\
\hline$C(114)$ & $C(115)$ & $1.35(3)$ & $C(411)$ & $C(412)$ & $1.38(2)$ \\
\hline$C(115)$ & $C(116)$ & $1.40(2)$ & $C(411)$ & $C(416)$ & $1.37(2)$ \\
\hline$C(121)$ & $C(122)$ & $1.41(2)$ & $C(412)$ & $C(413)$ & $1.43(2)$ \\
\hline$C(121)$ & $C(126)$ & $1.41(2)$ & $C(413)$ & $C(414)$ & $1.34(3)$ \\
\hline$C(122)$ & $C(123)$ & $1.36(3)$ & $C(414)$ & $C(415)$ & $1.41(3)$ \\
\hline$C(123)$ & $C(124)$ & $1.39(4)$ & $C(415)$ & $C(416)$ & $1.40(3)$ \\
\hline$C(124)$ & $C(125)$ & $1.33(4)$ & $C(421)$ & $C(422)$ & $1.36(2)$ \\
\hline$C(125)$ & $C(126)$ & $1.39(3)$ & $C(421)$ & $C(426)$ & $1.37(2)$ \\
\hline$C(211)$ & $C(212)$ & $1.38(2)$ & $C(422)$ & $C(423)$ & $1.38(2)$ \\
\hline$C(211)$ & $C(216)$ & $1.40(2)$ & $C(423)$ & $C(424)$ & $1.38(2)$ \\
\hline$C(424)$ & $C(425)$ & $1.38(2)$ & & & \\
\hline$C(425)$ & $C(426)$ & $1.37(2)$ & & & \\
\hline$C(511)$ & $C(512)$ & $1.39(2)$ & & & \\
\hline$C(511)$ & $C(516)$ & $1.39(2)$ & & & \\
\hline$C(512)$ & $C(513)$ & $1.35(2)$ & & & \\
\hline$C(513)$ & $C(514)$ & $1.39(3)$ & & & \\
\hline$C(514)$ & $C(515)$ & $1.35(3)$ & & & \\
\hline$C(515)$ & $C(516)$ & $1.44(3)$ & & & \\
\hline$C(611)$ & $C(612)$ & $1.39(2)$ & & & \\
\hline$C(611)$ & $C(616)$ & $1.38(2)$ & & & \\
\hline$C(612)$ & $C(613)$ & $1.37(2)$ & & & \\
\hline$C(613)$ & $C(614)$ & $1.38(2)$ & & & \\
\hline$C(614)$ & $C(615)$ & $1.38(2)$ & & & \\
\hline$C(615)$ & $C(616)$ & $1.37(2)$ & & & \\
\hline$C(621)$ & $C(622)$ & $1.39(2)$ & & & \\
\hline$C(621)$ & $C(626)$ & $1.39(2)$ & & & \\
\hline$C(622)$ & $C(623)$ & $1.38(2)$ & & & \\
\hline$C(623)$ & $C(624)$ & $1.36(2)$ & & & \\
\hline$C(624)$ & $C(625)$ & $1.39(2)$ & & & \\
\hline$C(625)$ & $C(626)$ & $1.39(2)$ & & & \\
\hline
\end{tabular}




$\begin{array}{lll}\mathrm{C}(711) & \mathrm{C}(712) & 1.48(5) \\ \mathrm{C}(711) & \mathrm{C}(713) & 1.58(4) \\ \mathrm{C}(721) & \mathrm{C}(722) & 1.50(6) \\ \mathrm{C}(721) & \mathrm{C}(723) & 1.61(6)\end{array}$

a Estimated standard deviations are given in parentheses.

Table S1-5. Bond Angles for Non-hydrogen Atoms of 2.2( $\left.\mathrm{CH}_{3}\right)_{2} \mathrm{CO}^{\mathrm{a}}$

\begin{tabular}{|c|c|c|c|c|c|c|c|}
\hline atom & atom & atom & angle & atom & atom & atom & angle \\
\hline$\overline{\operatorname{Pt}(2)}$ & $\operatorname{Pt}(1)$ & $\mathrm{P}(1)$ & $87.2(1)$ & $\operatorname{Pt}(2)$ & $\mathrm{P}(2)$ & $C(211)$ & $120.4(5)$ \\
\hline $\operatorname{Pt}(2)$ & $\operatorname{Pt}(1)$ & $\mathrm{P}(4)$ & 83.3(1) & $C(3)$ & $\mathrm{P}(2)$ & $C(4)$ & 103.1(7) \\
\hline $\operatorname{Pt}(2)$ & $\operatorname{Pt}(1)$ & $C(1)$ & $178.1(5)$ & $C(3)$ & $P(2)$ & $C(211)$ & 103.1(6) \\
\hline $\mathrm{P}(1)$ & $\operatorname{Pt}(1)$ & $\mathrm{P}(4)$ & $170.2(1)$ & $C(4)$ & $\mathrm{P}(2)$ & $C(211)$ & $105.4(7)$ \\
\hline $\mathrm{P}(1)$ & $\operatorname{Pt}(1)$ & $C(1)$ & $94.8(5)$ & $\operatorname{Pt}(3)$ & $P(3)$ & $C(4)$ & $116.3(5)$ \\
\hline $\mathrm{P}(4)$ & $\operatorname{Pt}(1)$ & $C(1)$ & $94.8(5)$ & $\operatorname{Pt}(3)$ & $P(3)$ & $C(311)$ & 113.6(5) \\
\hline $\operatorname{Pt}(1)$ & $\operatorname{Pt}(2)$ & $\operatorname{Pt}(3)$ & $178.92(3)$ & $\operatorname{Pt}(3)$ & $\mathrm{P}(3)$ & $C(321)$ & $112.5(6)$ \\
\hline $\operatorname{Pt}(1)$ & $\operatorname{Pt}(2)$ & $P(2)$ & $92.3(1)$ & $C(4)$ & $P(3)$ & $C(311)$ & 103.3(7) \\
\hline $\operatorname{Pt}(1)$ & $\operatorname{Pt}(2)$ & $P(5)$ & $92.8(1)$ & $C(4)$ & $\mathrm{P}(3)$ & $C(321)$ & $105.5(8)$ \\
\hline $\operatorname{Pt}(3)$ & $\operatorname{Pt}(2)$ & $P(2)$ & $87.3(1)$ & $C(311)$ & $P(3)$ & $C(321)$ & $104.6(8)$ \\
\hline $\operatorname{Pt}(3)$ & $\operatorname{Pt}(2)$ & $P(5)$ & $87.5(1)$ & $\operatorname{Pt}(1)$ & $\mathrm{P}(4)$ & $C(5)$ & $111.0(5)$ \\
\hline $\mathrm{P}(2)$ & $\operatorname{Pt}(2)$ & $P(5)$ & 173.3(1) & $\operatorname{Pt}(1)$ & $\mathrm{P}(4)$ & $C(411)$ & $113.1(5)$ \\
\hline $\operatorname{Pt}(2)$ & $\operatorname{Pt}(3)$ & $\mathrm{P}(3)$ & 88.7(1) & $\operatorname{Pt}(1)$ & $\mathrm{P}(4)$ & $C(421)$ & $116.4(6)$ \\
\hline $\operatorname{Pt}(2)$ & $\operatorname{Pt}(3)$ & $P(6)$ & 89.3(1) & $C(5)$ & $\mathrm{P}(4)$ & $C(411)$ & $106.0(7)$ \\
\hline $\operatorname{Pt}(2)$ & $\operatorname{Pt}(3)$ & $C(2)$ & $176.4(4)$ & $C(5)$ & $\mathrm{P}(4)$ & $C(421)$ & $106.3(7)$ \\
\hline$P(3)$ & $\operatorname{Pt}(3)$ & $P(6)$ & $177.0(1)$ & $C(411)$ & $\mathrm{P}(4)$ & $C(421)$ & 103.2(7) \\
\hline$P(3)$ & $\operatorname{Pt}(3)$ & $C(2)$ & $90.7(4)$ & $\operatorname{Pt}(2)$ & $P(5)$ & $C(5)$ & $115.5(5)$ \\
\hline$P(6)$ & $\operatorname{Pt}(3)$ & $C(2)$ & $91.2(4)$ & $\operatorname{Pt}(2)$ & $P(5)$ & $C(6)$ & $110.4(5)$ \\
\hline $\operatorname{Pt}(1)$ & $\mathrm{P}(1)$ & $C(3)$ & $109.8(5)$ & $\operatorname{Pt}(2)$ & $P(5)$ & $C(511)$ & $119.7(6)$ \\
\hline $\operatorname{Pt}(1)$ & $\mathrm{P}(1)$ & $C(111)$ & $114.3(5)$ & $C(5)$ & $P(5)$ & $C(6)$ & $100.9(7)$ \\
\hline $\operatorname{Pt}(1)$ & $\mathrm{P}(1)$ & $C(121)$ & $117.0(6)$ & $C(5)$ & $P(5)$ & $C(511)$ & $104.4(7)$ \\
\hline$C(3)$ & $\mathrm{P}(1)$ & $C(111)$ & $106.4(7)$ & $C(6)$ & $P(5)$ & $C(511)$ & $103.6(7)$ \\
\hline$C(3)$ & $P(1)$ & $C(121)$ & $106.6(7)$ & $\operatorname{Pt}(3)$ & $P(6)$ & $C(6)$ & $116.2(5)$ \\
\hline$C(111)$ & $\mathrm{P}(1)$ & $C(121)$ & $101.9(7)$ & $\operatorname{Pt}(3)$ & $P(6)$ & $C(611)$ & $110.9(5)$ \\
\hline $\operatorname{Pt}(2)$ & $P(2)$ & $C(3)$ & $116.6(5)$ & $\operatorname{Pt}(3)$ & $P(6)$ & $C(621)$ & 117.6(5) \\
\hline $\operatorname{Pt}(2)$ & $P(2)$ & $C(4)$ & 106.3(5) & $C(6)$ & $P(6)$ & $C(611)$ & $105.6(6)$ \\
\hline$C(6)$ & $\mathrm{P}(6)$ & $C(621)$ & $102.4(7)$ & $\mathrm{F}(23)$ & $\mathrm{P}(12)$ & $\mathrm{F}(24)$ & $91.3(7)$ \\
\hline$C(611)$ & $P(6)$ & $C(621)$ & $102.8(6)$ & $\mathrm{F}(23)$ & $P(12)$ & $F(25)$ & $87.1(9)$ \\
\hline $\mathrm{F}(11)$ & $\mathrm{P}(11)$ & $\mathrm{F}(12)$ & $95(1)$ & $\mathrm{F}(23)$ & $P(12)$ & $\mathrm{F}(26)$ & $176(1)$ \\
\hline $\mathrm{F}(11)$ & $\mathrm{P}(11)$ & $\mathrm{F}(13)$ & $88.3(8)$ & $\mathrm{F}(24)$ & $\mathrm{P}(12)$ & $\mathrm{F}(25)$ & $89.6(8)$ \\
\hline $\mathrm{F}(11)$ & $\mathrm{P}(11)$ & $\mathrm{F}(14)$ & $90.1(9)$ & $\mathrm{F}(24)$ & $P(12)$ & $F(26)$ & $88.1(7)$ \\
\hline $\mathrm{F}(11)$ & $\mathrm{P}(11)$ & $F(15)$ & $84.9(9)$ & $\mathrm{F}(25)$ & $\mathrm{P}(12)$ & $\mathrm{F}(26)$ & 89.2(9) \\
\hline $\mathrm{F}(11)$ & $\mathrm{P}(11)$ & $F(16)$ & $176(1)$ & $C(1)$ & $\mathrm{N}(1)$ & $C(11)$ & $173(2)$ \\
\hline$F(12)$ & $\mathrm{P}(11)$ & $\mathrm{F}(13)$ & $93(1)$ & $C(2)$ & $\mathrm{N}(2)$ & $C(21)$ & $175(2)$ \\
\hline$F(12)$ & $\mathrm{P}(11)$ & $\mathrm{F}(14)$ & $87(1)$ & $\operatorname{Pt}(1)$ & $C(1)$ & $\mathrm{N}(1)$ & $176(1)$ \\
\hline$F(12)$ & $\mathrm{P}(11)$ & $F(15)$ & $178(1)$ & $\operatorname{Pt}(3)$ & $C(2)$ & $\mathrm{N}(2)$ & $177(1)$ \\
\hline$F(12)$ & $\mathrm{P}(11)$ & $F(16)$ & $89(1)$ & $P(1)$ & $C(3)$ & $P(2)$ & $106.4(7)$ \\
\hline $\mathrm{F}(13)$ & $\mathrm{P}(11)$ & $\mathrm{F}(14)$ & $178(1)$ & $P(2)$ & $C(4)$ & $P(3)$ & $106.6(8)$ \\
\hline $\mathrm{F}(13)$ & $\mathrm{P}(11)$ & $\mathrm{F}(15)$ & $85(1)$ & $\mathrm{P}(4)$ & $C(5)$ & $P(5)$ & $104.8(7)$ \\
\hline $\mathrm{F}(13)$ & $\mathrm{P}(11)$ & $\mathrm{F}(16)$ & $91.1(9)$ & $P(5)$ & $C(6)$ & $P(6)$ & $108.8(7)$ \\
\hline $\mathrm{F}(14)$ & $P(11)$ & $\mathrm{F}(15)$ & $95(1)$ & $\mathrm{N}(1)$ & $C(11)$ & $C(12)$ & $119(1)$ \\
\hline $\mathrm{F}(14)$ & $\mathrm{P}(11)$ & $F(16)$ & $90(1)$ & $\mathrm{N}(1)$ & $C(11)$ & $C(16)$ & $118(1)$ \\
\hline $\mathrm{F}(15)$ & $\mathrm{P}(11)$ & $F(16)$ & $91(1)$ & $C(12)$ & $C(11)$ & $C(16)$ & $123(1)$ \\
\hline$F(21)$ & $\mathrm{P}(12)$ & $\mathrm{F}(22)$ & $90.0(7)$ & $C(11)$ & $C(12)$ & $C(13)$ & $117(2)$ \\
\hline $\mathrm{F}(21)$ & $\mathrm{P}(12)$ & $\mathrm{F}(23)$ & $89.8(9)$ & $C(11)$ & $C(12)$ & $C(17)$ & $119(1)$ \\
\hline $\mathrm{F}(21)$ & $\mathrm{P}(12)$ & $\mathrm{F}(24)$ & 89.1(8) & $C(13)$ & $C(12)$ & $C(17)$ & $124(2)$ \\
\hline $\mathrm{F}(21)$ & $\mathrm{P}(12)$ & $F(25)$ & 177(1) & $C(12)$ & $C(13)$ & $C(14)$ & $122(2)$ \\
\hline
\end{tabular}




\begin{tabular}{|c|c|c|c|c|c|c|c|}
\hline $\mathrm{F}(21)$ & $\mathrm{P}(12)$ & $\mathrm{F}(26)$ & $93.9(9)$ & $C(13)$ & $C(14)$ & $C(15)$ & $120(2)$ \\
\hline$F(22)$ & $\mathrm{P}(12)$ & $\mathrm{F}(23)$ & $89.4(7)$ & $C(13)$ & $C(14)$ & $C(18)$ & $118(2)$ \\
\hline $\mathrm{F}(22)$ & $\mathrm{P}(12)$ & $\mathrm{F}(24)$ & 178.9(8) & $C(15)$ & $C(14)$ & $C(18)$ & $123(2)$ \\
\hline $\mathrm{F}(22)$ & $\mathrm{P}(12)$ & $F(25)$ & $91.3(9)$ & $C(14)$ & $C(15)$ & $C(16)$ & $121(2)$ \\
\hline$F(22)$ & $\mathrm{P}(12)$ & $F(26)$ & 91.3(7) & $\mathrm{C}(11)$ & $C(16)$ & $C(15)$ & $117(2)$ \\
\hline$C(11)$ & $C(16)$ & $C(19)$ & $121(2)$ & $C(122)$ & $C(121)$ & $C(126)$ & $118(2)$ \\
\hline$C(15)$ & $C(16)$ & $C(19)$ & $122(2)$ & $C(121)$ & $C(122)$ & $C(123)$ & $121(2)$ \\
\hline $\mathrm{N}(2)$ & $C(21)$ & $C(22)$ & $118(2)$ & $C(122)$ & $C(123)$ & $C(124)$ & $120(2)$ \\
\hline $\mathrm{N}(2)$ & $C(21)$ & $C(26)$ & $120(2)$ & $C(123)$ & $C(124)$ & $C(125)$ & $120(3)$ \\
\hline$C(22)$ & $C(21)$ & $C(26)$ & $122(2)$ & $C(124)$ & $C(125)$ & $C(126)$ & $122(3)$ \\
\hline$C(21)$ & $C(22)$ & $C(23)$ & 119(2) & $C(121)$ & $C(126)$ & $C(125)$ & $119(2)$ \\
\hline$C(21)$ & $C(22)$ & $C(27)$ & $121(2)$ & $\mathrm{P}(2)$ & $C(211)$ & $C(212)$ & $124(1)$ \\
\hline$C(23)$ & $C(22)$ & $C(27)$ & $120(2)$ & $\mathrm{P}(2)$ & $C(211)$ & $C(216)$ & $119(1)$ \\
\hline$C(22)$ & $C(23)$ & $C(24)$ & 119(2) & $C(212)$ & $C(211)$ & $C(216)$ & $117(1)$ \\
\hline$C(23)$ & $C(24)$ & $C(25)$ & $123(2)$ & $C(211)$ & $C(212)$ & $C(213)$ & $121(1)$ \\
\hline$C(23)$ & $C(24)$ & $C(28)$ & $121(2)$ & $C(212)$ & $C(213)$ & $C(214)$ & $120(2)$ \\
\hline$C(25)$ & $C(24)$ & $C(28)$ & $116(2)$ & $C(213)$ & $C(214)$ & $C(215)$ & $119(2)$ \\
\hline$C(24)$ & $C(25)$ & $C(26)$ & $120(2)$ & $C(214)$ & $C(215)$ & $C(216)$ & $120(2)$ \\
\hline$C(21)$ & $C(26)$ & $C(25)$ & $117(2)$ & $C(211)$ & $C(216)$ & $C(215)$ & $122(2)$ \\
\hline$C(21)$ & $C(26)$ & $C(29)$ & $120(2)$ & $\mathrm{P}(3)$ & $C(311)$ & $C(312)$ & $120(1)$ \\
\hline$C(25)$ & $C(26)$ & $C(29)$ & $122(2)$ & $\mathrm{P}(3)$ & $C(311)$ & $C(316)$ & $123(1)$ \\
\hline $\mathrm{P}(1)$ & $C(111)$ & $C(112)$ & 121(1) & $C(312)$ & $C(311)$ & $C(316)$ & $117(2)$ \\
\hline $\mathrm{P}(1)$ & $C(111)$ & $C(116)$ & 119(1) & $C(311)$ & $C(312)$ & $C(313)$ & $122(2)$ \\
\hline$C(112)$ & $C(111)$ & $C(116)$ & $120(2)$ & $C(312)$ & $C(313)$ & $C(314)$ & $121(2)$ \\
\hline$C(111)$ & $C(112)$ & $C(113)$ & $118(2)$ & $C(313)$ & $C(314)$ & $C(315)$ & $118(2)$ \\
\hline$C(112)$ & $C(113)$ & $C(114)$ & 121(2) & $C(314)$ & $C(315)$ & $C(316)$ & $121(2)$ \\
\hline$C(113)$ & $C(114)$ & $C(115)$ & 121(2) & $C(311)$ & $C(316)$ & $C(315)$ & $122(2)$ \\
\hline$C(114)$ & $C(115)$ & $C(116)$ & 119(2) & $\mathrm{P}(3)$ & $C(321)$ & $C(322)$ & $124(2)$ \\
\hline$C(111)$ & $C(116)$ & $C(115)$ & $122(2)$ & $\mathrm{P}(3)$ & $C(321)$ & $C(326)$ & $118(2)$ \\
\hline $\mathrm{P}(1)$ & $C(121)$ & $C(122)$ & 123(1) & $C(322)$ & $C(321)$ & $C(326)$ & $117(2)$ \\
\hline $\mathrm{P}(1)$ & $C(121)$ & $C(126)$ & 119(1) & $C(321)$ & $C(322)$ & $C(323)$ & $125(2)$ \\
\hline$C(322)$ & $C(323)$ & $C(324)$ & $120(3)$ & $C(514)$ & $C(515)$ & $C(516)$ & $120(2)$ \\
\hline$C(323)$ & C(324) & $C(325)$ & $118(2)$ & $C(511)$ & $C(516)$ & $C(515)$ & $117(2)$ \\
\hline$C(324)$ & $C(325)$ & $C(326)$ & $120(2)$ & $\mathrm{P}(6)$ & $C(611)$ & $C(612)$ & $120(1)$ \\
\hline$C(321)$ & $C(326)$ & $C(325)$ & $120(3)$ & $P(6)$ & $C(611)$ & $C(616)$ & $122(1)$ \\
\hline $\mathrm{P}(4)$ & $C(411)$ & $C(412)$ & 119(1) & $C(612)$ & $C(611)$ & $C(616)$ & 117(1) \\
\hline $\mathrm{P}(4)$ & $C(411)$ & $C(416)$ & $120(1)$ & $C(611)$ & $C(612)$ & $C(613)$ & $122(1)$ \\
\hline$C(412)$ & $C(411)$ & $C(416)$ & $121(2)$ & $C(612)$ & $C(613)$ & $C(614)$ & $120(2)$ \\
\hline$C(411)$ & $C(412)$ & $C(413)$ & $120(2)$ & $C(613)$ & $C(614)$ & $C(615)$ & $119(1)$ \\
\hline$C(412)$ & $C(413)$ & $C(414)$ & $118(2)$ & $C(614)$ & $C(615)$ & $C(616)$ & $120(2)$ \\
\hline$C(413)$ & $C(414)$ & $C(415)$ & $123(2)$ & $C(611)$ & $C(616)$ & $C(615)$ & $121(1)$ \\
\hline$C(414)$ & $C(415)$ & $C(416)$ & 117(2) & $P(6)$ & $C(621)$ & $C(622)$ & 121(1) \\
\hline$C(411)$ & $C(416)$ & $C(415)$ & $121(2)$ & $P(6)$ & $C(621)$ & $C(626)$ & $120(1)$ \\
\hline $\mathrm{P}(4)$ & $C(421)$ & $C(422)$ & $120(1)$ & $C(622)$ & $C(621)$ & $C(626)$ & $119(1)$ \\
\hline $\mathrm{P}(4)$ & $C(421)$ & $C(426)$ & 120(1) & $C(621)$ & $C(622)$ & $C(623)$ & 121(1) \\
\hline$C(422)$ & $C(421)$ & $C(426)$ & 120(1) & $C(622)$ & $C(623)$ & $C(624)$ & $120(2)$ \\
\hline$C(421)$ & $C(422)$ & $C(423)$ & $121(2)$ & $C(623)$ & $C(624)$ & $C(625)$ & $121(1)$ \\
\hline$C(422)$ & $C(423)$ & $C(424)$ & $120(2)$ & $C(624)$ & $C(625)$ & $C(626)$ & 119(2) \\
\hline$C(423)$ & $C(424)$ & $C(425)$ & $118(2)$ & $C(621)$ & $C(626)$ & $C(625)$ & $120(1)$ \\
\hline$C(424)$ & $C(425)$ & $C(426)$ & $122(2)$ & $\mathrm{O}(701)$ & $C(711)$ & $C(712)$ & $125(3)$ \\
\hline$C(421)$ & $C(426)$ & $C(425)$ & $119(2)$ & $\mathrm{O}(701)$ & $C(711)$ & $C(713)$ & $112(4)$ \\
\hline$P(5)$ & $C(511)$ & $C(512)$ & 119(1) & $C(712)$ & $C(711)$ & $C(713)$ & $123(3)$ \\
\hline$P(5)$ & $C(511)$ & $C(516)$ & $120(1)$ & $\mathrm{O}(702)$ & $C(721)$ & $C(722)$ & $122(4)$ \\
\hline$C(512)$ & $C(511)$ & $C(516)$ & 121(2) & $\mathrm{O}(702)$ & $C(721)$ & $C(723)$ & 107(5) \\
\hline$C(511)$ & $C(512)$ & $C(513)$ & 121(2) & $C(722)$ & $C(721)$ & $C(723)$ & $130(3)$ \\
\hline$C(512)$ & $C(513)$ & $C(514)$ & $119(2)$ & & & & \\
\hline
\end{tabular}


$\mathrm{C}(513) \quad \mathrm{C}(514) \quad \mathrm{C}(515) \quad 121(2)$

a Estimated deviations are given in parentheses. 
Table S2-1. Crystallographic and Experimental Data for $3 \cdot 2.5 \mathrm{CH}_{2} \mathrm{Cl}_{2}$

\begin{tabular}{|c|c|}
\hline formula & $\mathrm{C}_{80.5} \mathrm{H}_{81} \mathrm{~N}_{2} \mathrm{P}_{8} \mathrm{~F}_{12} \mathrm{Pt}_{3} \mathrm{Cl}_{5}$ \\
\hline formula weight & 2314.84 \\
\hline color & orange \\
\hline size, $\mathrm{mm}$ & $0.35 \times 0.30 \times 0.20$ \\
\hline cryst. system & monoclinic \\
\hline space group & $P 21$ (No. 4) \\
\hline no. refs used for cell determination & $25\left(20<2 \theta<30^{\circ}\right)$ \\
\hline lattice const. & $\begin{array}{l}a=15.167(4) \AA \\
b=13.976(3) \AA \\
c=21.634(3) \AA \\
\beta=108.01(1){ }^{\circ} \\
V=4361(1) \AA^{3}\end{array}$ \\
\hline Z & 2 \\
\hline$D_{\text {calc }}, \mathrm{g} \mathrm{cm}^{-3}$ & 1.763 \\
\hline$\mu(\mathrm{Mo} K \alpha), \mathrm{cm}^{-1}$ & 51.49 (trans. $0.76 \sim 1.00$ ) \\
\hline \multirow{2}{*}{\multicolumn{2}{|c|}{ An absorption correction was applied ( $\psi$-scan method). }} \\
\hline & 2250 \\
\hline diffractometer & Rigaku AFC7R (50 kV, $200 \mathrm{~mA})$ \\
\hline radiation & $\operatorname{Mo} K \alpha(\lambda=0.71069 \AA)$ \\
\hline monochromator & graphite \\
\hline temp, ${ }^{\circ} \mathrm{C}$ & -118 \\
\hline $2 \theta$ range, $^{\circ}$ & $4<2 \theta<50$ \\
\hline $\mathrm{h}, \mathrm{k}, \mathrm{l}$ range & $+\mathrm{h},+\mathrm{k}, \pm 1$ \\
\hline scan speed, ${ }^{\circ} \min ^{-1}$ & $\begin{array}{l}\omega-2 \theta \\
8\end{array}$ \\
\hline scan width & $1.68+0.30 \tan \theta$ \\
\hline std. reflections & 3 every 150 refs \\
\hline solution & Direct Methods (SIR92) \\
\hline \multirow[t]{2}{*}{ refinement } & full-matrix least-squares \\
\hline & $\begin{array}{l}\text { The } \mathrm{Pt}, \mathrm{Cl} \text {, and } \mathrm{P} \text { atoms were refined with } \\
\text { anisotropic temperature factors and other } \\
\text { non-hydrogen atoms, with isotropic ones. }\end{array}$ \\
\hline hydrogen atoms & $\begin{array}{l}\text { All } \mathrm{H} \text { atoms except for solvent molecules } \\
\text { were calcd with } \mathrm{C}-\mathrm{H}=0.95 \AA \text {, and were not refined. }\end{array}$ \\
\hline function minimized & $\Sigma w\left(\left|F_{o}\right|-\left|F_{c}\right|\right)^{2}$ \\
\hline weighting scheme & $1 / \sigma^{2}\left(F_{0}\right)$ \\
\hline anomalous dispersion & all non-hydrogen atoms \\
\hline no. unique reflections & 7936 \\
\hline$R_{\text {int }}$ & 0.111 \\
\hline no. obsd. unique reflections & $5363(I>2 \sigma(I))$ \\
\hline$p$-factor & 0.05 \\
\hline no. variables & 540 \\
\hline data / param ratio & 9.93 \\
\hline$R^{\mathrm{a}}$ & 0.058 \\
\hline$R_{\mathrm{W}} \mathrm{b}$ & 0.064 \\
\hline $\mathrm{GOF}^{\mathrm{C}}$ & 1.36 \\
\hline$\rho_{\max }, \mathrm{e}^{-3}$ & $1.41,-1.18$ \\
\hline
\end{tabular}

Table S2-2. Final Positional Parameters and $B_{\text {eq }}$ for $3 \cdot 2.5 \mathrm{CH}_{2} \mathrm{Cl}_{2} \mathrm{a}, \mathrm{b}, \mathrm{c}$

\begin{tabular}{lcccl}
\hline atom & $x$ & $y$ & $z$ & $B_{\mathrm{eq}}$ \\
\hline $\mathrm{Pt}(1)$ & $0.69131(6)$ & $0.300(2)$ & $0.32945(4)$ & $2.44(2)$
\end{tabular}




\begin{tabular}{|c|c|c|c|c|}
\hline $\operatorname{Pt}(2)$ & $0.34686(6)$ & $0.184(2)$ & $0.18835(4)$ & $2.16(2)$ \\
\hline $\operatorname{Pt}(3)$ & $0.51589(7)$ & $0.247(2)$ & $0.25904(5)$ & $2.07(2)$ \\
\hline $\mathrm{Cl}(1)$ & $0.7999(8)$ & $0.078(3)$ & $0.2687(7)$ & $12.5(5)$ \\
\hline $\mathrm{Cl}(2)$ & $0.8219(10)$ & $0.045(3)$ & $0.1435(9)$ & $15.3(7)$ \\
\hline $\mathrm{Cl}(3)$ & $0.265(1)$ & $0.264(3)$ & $0.7380(9)$ & $15.2(7)$ \\
\hline $\mathrm{Cl}(4)$ & $0.1345(9)$ & $0.191(3)$ & $0.6224(8)$ & $15.5(6)$ \\
\hline $\mathrm{Cl}(5)^{\mathrm{d}}$ & $0.006(3)$ & $0.330(4)$ & $0.772(1)$ & $22(1)$ \\
\hline$C l(6)^{d}$ & $0.873(1)$ & $0.231(3)$ & $0.776(1)$ & $11.0(8)$ \\
\hline $\mathrm{P}(1)$ & $0.6532(4)$ & $0.225(2)$ & $0.4107(3)$ & $2.5(1)$ \\
\hline $\mathrm{P}(2)$ & $0.4598(4)$ & $0.265(2)$ & $0.3441(3)$ & $2.2(1)$ \\
\hline $\mathrm{P}(3)$ & $0.3308(4)$ & $0.109(2)$ & $0.2780(3)$ & $2.3(1)$ \\
\hline $\mathrm{P}(4)$ & $0.6988(4)$ & $0.369(2)$ & $0.2351(3)$ & $2.4(1)$ \\
\hline $\mathrm{P}(5)$ & $0.5543(4)$ & $0.235(2)$ & $0.1671(3)$ & $2.3(1)$ \\
\hline $\mathrm{P}(6)$ & $0.3580(4)$ & $0.281(2)$ & $0.1060(3)$ & $2.4(1)$ \\
\hline $\mathrm{P}(11)$ & $0.7314(6)$ & $0.326(2)$ & $-0.0161(4)$ & $4.9(2)$ \\
\hline $\mathrm{P}(12)$ & $0.5990(6)$ & $0.601(2)$ & $0.4505(3)$ & $3.8(2)$ \\
\hline $\mathrm{F}(11)$ & $0.750(1)$ & $0.236(3)$ & $0.0320(9)$ & $7.0(4)$ \\
\hline $\mathrm{F}(12)$ & $0.675(1)$ & $0.375(3)$ & $0.0266(8)$ & $5.3(4)$ \\
\hline $\mathrm{F}(13)$ & $0.825(1)$ & $0.371(3)$ & $0.0321(9)$ & $6.7(4)$ \\
\hline $\mathrm{F}(14)$ & $0.787(1)$ & $0.279(3)$ & $-0.0589(8)$ & $5.9(4)$ \\
\hline$F(15)$ & $0.711(1)$ & $0.418(3)$ & $-0.0617(9)$ & $5.8(4)$ \\
\hline $\mathrm{F}(16)$ & $0.637(1)$ & $0.282(3)$ & $-0.0626(9)$ & $6.6(4)$ \\
\hline $\mathrm{F}(21)$ & $0.651(2)$ & $0.563(3)$ & $0.520(1)$ & $9.9(6)$ \\
\hline$F(22)$ & $0.553(1)$ & $0.681(3)$ & $0.4807(9)$ & $7.8(5)$ \\
\hline$F(23)$ & $0.682(2)$ & $0.670(4)$ & $0.450(2)$ & $14.3(8)$ \\
\hline $\mathrm{F}(24)$ & $0.644(1)$ & $0.519(3)$ & $0.420(1)$ & $7.7(5)$ \\
\hline$F(25)$ & $0.554(1)$ & $0.648(3)$ & $0.383(1)$ & $8.0(5)$ \\
\hline$F(26)$ & $0.515(3)$ & $0.538(4)$ & $0.445(2)$ & $17.9(10)$ \\
\hline $\mathrm{N}(1)$ & $0.146(2)$ & $0.123(3)$ & $0.101(1)$ & $5.1(6)$ \\
\hline $\mathrm{N}(2)$ & $0.881(2)$ & $0.365(3)$ & $0.425(1)$ & $3.9(5)$ \\
\hline$C(1)$ & $0.226(2)$ & $0.141(3)$ & $0.133(1)$ & $2.6(5)$ \\
\hline$C(2)$ & $0.807(2)$ & $0.345(3)$ & $0.379(1)$ & $2.5(5)$ \\
\hline$C(3)$ & $0.549(2)$ & $0.279(3)$ & $0.421(1)$ & $3.0(5)$ \\
\hline$C(4)$ & $0.402(1)$ & $0.155(3)$ & $0.3568(9)$ & $1.6(4)$ \\
\hline$C(5)$ & $0.666(2)$ & $0.282(3)$ & $0.170(1)$ & $3.1(5)$ \\
\hline$C(6)$ & $0.475(2)$ & $0.310(3)$ & $0.105(1)$ & $2.9(5)$ \\
\hline$C(11)$ & $0.051(2)$ & $0.107(4)$ & $0.059(2)$ & $5.9(7)$ \\
\hline$C(12)$ & $0.025(3)$ & $0.002(4)$ & $0.083(2)$ & $10.4(10)$ \\
\hline$C(13)$ & $-0.010(2)$ & $0.168(4)$ & $0.079(2)$ & $7.4(8)$ \\
\hline$C(14)$ & $0.051(4)$ & $0.151(6)$ & $-0.009(3)$ & $15(1)$ \\
\hline$C(21)$ & $0.965(2)$ & $0.405(3)$ & $0.470(2)$ & $5.4(7)$ \\
\hline$C(22)$ & $1.041(3)$ & $0.349(4)$ & $0.466(2)$ & $7.0(8)$ \\
\hline$C(23)$ & $1.130(2)$ & $0.380(3)$ & $0.517(2)$ & $5.5(7)$ \\
\hline$C(24)$ & $1.126(2)$ & $0.445(4)$ & $0.564(2)$ & $6.4(8)$ \\
\hline$C(25)$ & $1.042(3)$ & $0.483(4)$ & $0.557(2)$ & $7.5(8)$ \\
\hline$C(26)$ & $0.962(2)$ & $0.461(3)$ & $0.513(1)$ & $4.5(6)$ \\
\hline$C(27)$ & $1.047(3)$ & $0.273(4)$ & $0.422(2)$ & $10.2(10)$ \\
\hline$C(28)$ & $0.874(3)$ & $0.517(4)$ & $0.514(2)$ & $8.4(9)$ \\
\hline$C(111)$ & $0.745(1)$ & $0.228(3)$ & $0.491(1)$ & $2.2(4)$ \\
\hline$C(112)$ & $0.815(2)$ & $0.165(3)$ & $0.503(1)$ & $5.0(6)$ \\
\hline$C(113)$ & $0.889(2)$ & $0.166(3)$ & $0.559(1)$ & $4.4(6)$ \\
\hline$C(114)$ & $0.890(2)$ & $0.229(4)$ & $0.605(2)$ & $6.6(8)$ \\
\hline$C(115)$ & $0.822(3)$ & $0.307(4)$ & $0.589(2)$ & $9.1(9)$ \\
\hline$C(116)$ & $0.744(2)$ & $0.296(4)$ & $0.533(2)$ & $6.2(7)$ \\
\hline$C(121)$ & $0.631(1)$ & $0.099(3)$ & $0.400(1)$ & $2.3(4)$ \\
\hline$C(122)$ & $0.615(2)$ & $0.040(3)$ & $0.451(1)$ & $3.3(5)$ \\
\hline$C(123)$ & $0.600(2)$ & $-0.055(3)$ & $0.443(1)$ & $3.8(5)$ \\
\hline
\end{tabular}




\begin{tabular}{|c|c|c|c|c|}
\hline$C(124)$ & $0.597(2)$ & $-0.101(3)$ & $0.383(1)$ & $4.0(6)$ \\
\hline$C(125)$ & $0.616(2)$ & $-0.044(3)$ & $0.335(2)$ & $5.4(7)$ \\
\hline$C(126)$ & $0.627(2)$ & $0.052(3)$ & $0.345(1)$ & $3.1(5)$ \\
\hline$C(211)$ & $0.379(1)$ & $0.359(3)$ & $0.341(1)$ & $2.2(4)$ \\
\hline$C(212)$ & $0.335(2)$ & $0.364(3)$ & $0.385(1)$ & $2.8(4)$ \\
\hline$C(213)$ & $0.266(2)$ & $0.442(3)$ & $0.385(1)$ & $4.4(6)$ \\
\hline$C(214)$ & $0.257(2)$ & $0.505(3)$ & $0.336(1)$ & $4.3(6)$ \\
\hline$C(215)$ & $0.305(2)$ & $0.504(3)$ & $0.292(1)$ & $4.9(6)$ \\
\hline$C(216)$ & $0.362(2)$ & $0.429(3)$ & $0.295(1)$ & $2.9(5)$ \\
\hline$C(311)$ & $0.351(1)$ & $-0.020(3)$ & $0.284(1)$ & $2.3(4)$ \\
\hline$C(312)$ & $0.365(2)$ & $-0.072(3)$ & $0.340(1)$ & $2.7(4)$ \\
\hline$C(313)$ & $0.377(2)$ & $-0.168(3)$ & $0.342(1)$ & $4.8(6)$ \\
\hline$C(314)$ & $0.370(2)$ & $-0.215(3)$ & $0.284(1)$ & $4.9(6)$ \\
\hline$C(315)$ & $0.355(2)$ & $-0.168(3)$ & $0.228(1)$ & $4.1(6)$ \\
\hline$C(316)$ & $0.344(2)$ & $-0.067(3)$ & $0.227(1)$ & $3.4(5)$ \\
\hline$C(321)$ & $0.210(2)$ & $0.126(3)$ & $0.278(1)$ & $3.2(5)$ \\
\hline$C(322)$ & $0.156(2)$ & $0.053(3)$ & $0.291(2)$ & $6.7(8)$ \\
\hline$C(323)$ & $0.061(3)$ & $0.067(4)$ & $0.287(2)$ & $8.4(9)$ \\
\hline$C(324)$ & $0.028(2)$ & $0.156(3)$ & $0.277(2)$ & $6.5(8)$ \\
\hline$C(325)$ & $0.082(2)$ & $0.234(3)$ & $0.270(2)$ & $5.7(7)$ \\
\hline$C(326)$ & $0.175(2)$ & $0.219(3)$ & $0.270(1)$ & $3.4(5)$ \\
\hline$C(411)$ & $0.809(1)$ & $0.418(3)$ & $0.230(1)$ & $2.1(4)$ \\
\hline$C(412)$ & $0.857(2)$ & $0.479(3)$ & $0.277(1)$ & $4.1(6)$ \\
\hline$C(413)$ & $0.942(2)$ & $0.522(3)$ & $0.274(2)$ & $5.2(6)$ \\
\hline$C(414)$ & $0.981(2)$ & $0.497(3)$ & $0.228(2)$ & $5.6(7)$ \\
\hline$C(415)$ & $0.935(2)$ & $0.426(3)$ & $0.183(2)$ & $5.8(7)$ \\
\hline$C(416)$ & $0.852(2)$ & $0.387(3)$ & $0.187(1)$ & $2.6(4)$ \\
\hline$C(421)$ & $0.626(1)$ & $0.479(3)$ & $0.207(1)$ & $2.1(4)$ \\
\hline$C(422)$ & $0.581(2)$ & $0.512(3)$ & $0.251(1)$ & $3.0(5)$ \\
\hline C(423) & $0.532(2)$ & $0.598(3)$ & $0.233(1)$ & $4.8(6)$ \\
\hline$C(424)$ & $0.524(2)$ & $0.644(3)$ & $0.177(1)$ & $4.3(6)$ \\
\hline$C(425)$ & $0.566(2)$ & $0.605(3)$ & $0.135(1)$ & $3.9(5)$ \\
\hline$C(426)$ & $0.619(2)$ & $0.522(3)$ & $0.150(1)$ & $2.8(5)$ \\
\hline$C(511)$ & $0.552(2)$ & $0.118(3)$ & $0.131(1)$ & $2.6(4)$ \\
\hline$C(512)$ & $0.520(2)$ & $0.037(3)$ & $0.157(1)$ & $2.6(4)$ \\
\hline$C(513)$ & $0.517(2)$ & $-0.054(3)$ & $0.130(1)$ & $3.9(5)$ \\
\hline$C(514)$ & $0.535(2)$ & $-0.067(3)$ & $0.074(1)$ & $4.8(6)$ \\
\hline$C(515)$ & $0.570(2)$ & $0.018(3)$ & $0.048(1)$ & $4.2(6)$ \\
\hline$C(516)$ & $0.575(2)$ & $0.102(3)$ & $0.073(1)$ & $3.4(5)$ \\
\hline$C(611)$ & $0.300(1)$ & $0.393(3)$ & $0.1088(9)$ & $1.5(4)$ \\
\hline$C(612)$ & $0.209(2)$ & $0.390(3)$ & $0.110(1)$ & $3.9(5)$ \\
\hline$C(613)$ & $0.159(2)$ & $0.474(3)$ & $0.113(1)$ & $4.9(6)$ \\
\hline C(614) & $0.201(2)$ & $0.556(3)$ & $0.109(2)$ & $6.0(7)$ \\
\hline$C(615)$ & $0.289(2)$ & $0.561(3)$ & $0.108(1)$ & $4.1(6)$ \\
\hline$C(616)$ & $0.341(2)$ & $0.483(3)$ & $0.107(1)$ & $3.8(5)$ \\
\hline$C(621)$ & $0.304(1)$ & $0.238(3)$ & $0.025(1)$ & $2.2(4)$ \\
\hline$C(622)$ & $0.296(2)$ & $0.139(3)$ & $0.012(1)$ & $2.9(5)$ \\
\hline$C(623)$ & $0.256(2)$ & $0.107(3)$ & $-0.050(1)$ & $3.6(5)$ \\
\hline$C(624)$ & $0.221(2)$ & $0.169(3)$ & $-0.100(2)$ & $5.6(7)$ \\
\hline$C(625)$ & $0.230(2)$ & $0.262(3)$ & $-0.089(1)$ & $5.1(6)$ \\
\hline$C(626)$ & $0.267(2)$ & $0.299(3)$ & $-0.028(1)$ & $3.9(5)$ \\
\hline$C(701)$ & $0.779(3)$ & $-0.007(4)$ & $0.196(2)$ & 7.3(8) \\
\hline $\mathrm{C}(702)^{\mathrm{e}}$ & $0.157(9)$ & $0.26(1)$ & $0.715(6)$ & $7(1)$ \\
\hline$C(703)^{f}$ & $0.250(3)$ & $0.216(4)$ & $0.662(2)$ & $5.3(9)$ \\
\hline$C(704)^{d}$ & $-0.080(7)$ & $0.300(8)$ & $0.813(5)$ & $10(1)$ \\
\hline $\mathrm{H}(1)$ & -0.0323 & -0.0218 & 0.0624 & 11.5 \\
\hline $\mathrm{H}(2)$ & 0.0717 & -0.0470 & 0.0732 & 11.5 \\
\hline
\end{tabular}




\begin{tabular}{|c|c|c|c|c|}
\hline $\mathrm{H}(3)$ & 0.0412 & -0.0042 & 0.1299 & 11.5 \\
\hline $\mathrm{H}(4)$ & -0.0008 & 0.1590 & 0.1276 & 9.0 \\
\hline $\mathrm{H}(5)$ & -0.0102 & 0.2294 & 0.0702 & 9.0 \\
\hline $\mathrm{H}(6)$ & -0.0768 & 0.1419 & 0.0611 & 9.0 \\
\hline $\mathrm{H}(7)$ & 0.0756 & 0.1986 & -0.0080 & 16.7 \\
\hline $\mathrm{H}(8)$ & 0.0874 & 0.0913 & -0.0234 & 16.7 \\
\hline $\mathrm{H}(9)$ & -0.0117 & 0.1350 & -0.0383 & 16.7 \\
\hline $\mathrm{H}(10)$ & 0.5379 & 0.2438 & 0.4590 & 4.8 \\
\hline $\mathrm{H}(11)$ & 0.5653 & 0.3407 & 0.4344 & 4.8 \\
\hline $\mathrm{H}(12)$ & 0.4469 & 0.1013 & 0.3801 & 1.9 \\
\hline $\mathrm{H}(13)$ & 0.3623 & 0.1616 & 0.3842 & 1.9 \\
\hline $\mathrm{H}(14)$ & 0.6667 & 0.3017 & 0.1297 & 2.6 \\
\hline $\mathrm{H}(15)$ & 0.7133 & 0.2221 & 0.1796 & 2.6 \\
\hline $\mathrm{H}(16)$ & 0.4894 & 0.3724 & 0.1154 & 3.4 \\
\hline $\mathrm{H}(17)$ & 0.4832 & 0.2944 & 0.0628 & 3.4 \\
\hline $\mathrm{H}(18)$ & 0.9810 & 0.2537 & 0.3941 & 10.3 \\
\hline $\mathrm{H}(19)$ & 1.0806 & 0.2853 & 0.3963 & 10.3 \\
\hline $\mathrm{H}(20)$ & 1.0675 & 0.2084 & 0.4448 & 10.3 \\
\hline $\mathrm{H}(21)$ & 0.8835 & 0.5468 & 0.5529 & 9.6 \\
\hline $\mathrm{H}(22)$ & 0.8474 & 0.5482 & 0.4772 & 9.6 \\
\hline $\mathrm{H}(23)$ & 0.8228 & 0.4627 & 0.5151 & 9.6 \\
\hline $\mathrm{H}(24)$ & 1.1844 & 0.3481 & 0.5144 & 7.6 \\
\hline $\mathrm{H}(25)$ & 1.1824 & 0.4588 & 0.6005 & 7.6 \\
\hline $\mathrm{H}(26)$ & 1.0349 & 0.5306 & 0.5896 & 8.5 \\
\hline $\mathrm{H}(27)$ & 0.8076 & 0.1085 & 0.4706 & 4.9 \\
\hline $\mathrm{H}(28)$ & 0.9403 & 0.1170 & 0.5634 & 6.0 \\
\hline $\mathrm{H}(29)$ & 0.9375 & 0.2228 & 0.6487 & 6.2 \\
\hline $\mathrm{H}(30)$ & 0.8199 & 0.3532 & 0.6138 & 12.1 \\
\hline $\mathrm{H}(31)$ & 0.6853 & 0.3266 & 0.5197 & 7.1 \\
\hline $\mathrm{H}(32)$ & 0.6155 & 0.0662 & 0.4890 & 3.4 \\
\hline $\mathrm{H}(33)$ & 0.5954 & -0.0997 & 0.4798 & 5.2 \\
\hline $\mathrm{H}(34)$ & 0.5861 & -0.1755 & 0.3753 & 4.0 \\
\hline $\mathrm{H}(35)$ & 0.6144 & -0.0775 & 0.2956 & 7.0 \\
\hline $\mathrm{H}(36)$ & 0.6345 & 0.0873 & 0.3101 & 3.9 \\
\hline $\mathrm{H}(37)$ & 0.3450 & 0.3074 & 0.4207 & 3.8 \\
\hline $\mathrm{H}(38)$ & 0.2348 & 0.4371 & 0.4165 & 5.3 \\
\hline $\mathrm{H}(39)$ & 0.2124 & 0.5485 & 0.3353 & 5.8 \\
\hline $\mathrm{H}(40)$ & 0.2950 & 0.5444 & 0.2562 & 6.2 \\
\hline $\mathrm{H}(41)$ & 0.3921 & 0.4151 & 0.2601 & 4.1 \\
\hline $\mathrm{H}(42)$ & 0.1841 & -0.0154 & 0.2991 & 7.9 \\
\hline $\mathrm{H}(43)$ & 0.0182 & 0.0075 & 0.2926 & 11.9 \\
\hline $\mathrm{H}(44)$ & -0.0383 & 0.1687 & 0.2750 & 7.8 \\
\hline $\mathrm{H}(45)$ & 0.0582 & 0.2965 & 0.2620 & 7.1 \\
\hline $\mathrm{H}(46)$ & 0.2202 & 0.2634 & 0.2645 & 3.5 \\
\hline $\mathrm{H}(47)$ & 0.3574 & -0.0445 & 0.3784 & 2.7 \\
\hline $\mathrm{H}(48)$ & 0.4034 & -0.2087 & 0.3863 & 7.0 \\
\hline $\mathrm{H}(49)$ & 0.3772 & -0.2934 & 0.2859 & 4.8 \\
\hline $\mathrm{H}(50)$ & 0.3407 & -0.2112 & 0.1859 & 4.7 \\
\hline $\mathrm{H}(51)$ & 0.3295 & -0.0391 & 0.1846 & 3.7 \\
\hline $\mathrm{H}(52)$ & 0.8333 & 0.4873 & 0.3132 & 4.4 \\
\hline $\mathrm{H}(53)$ & 0.9665 & 0.5721 & 0.3031 & 6.1 \\
\hline $\mathrm{H}(54)$ & 1.0425 & 0.5128 & 0.2273 & 5.9 \\
\hline $\mathrm{H}(55)$ & 0.9534 & 0.3979 & 0.1465 & 6.5 \\
\hline $\mathrm{H}(56)$ & 0.8223 & 0.3315 & 0.1547 & 3.4 \\
\hline $\mathrm{H}(57)$ & 0.5864 & 0.4755 & 0.2898 & 3.2 \\
\hline $\mathrm{H}(58)$ & 0.4998 & 0.6192 & 0.2600 & 6.4 \\
\hline $\mathrm{H}(59)$ & 0.4872 & 0.6955 & 0.1651 & 4.4 \\
\hline $\mathrm{H}(60)$ & 0.5607 & 0.6261 & 0.0948 & 3.9 \\
\hline
\end{tabular}




$\begin{array}{llrrr}\mathrm{H}(61) & 0.6492 & 0.4866 & 0.1192 & 3.1 \\ \mathrm{H}(62) & 0.5017 & 0.0409 & 0.1963 & 3.0 \\ \mathrm{H}(63) & 0.4871 & -0.1096 & 0.1503 & 4.4 \\ \mathrm{H}(64) & 0.5476 & -0.1397 & 0.0578 & 7.0 \\ \mathrm{H}(65) & 0.5761 & 0.0018 & 0.0033 & 4.6 \\ \mathrm{H}(66) & 0.5946 & 0.1506 & 0.0495 & 5.1 \\ \mathrm{H}(67) & 0.3248 & 0.0886 & 0.0497 & 3.0 \\ \mathrm{H}(68) & 0.2514 & 0.0297 & -0.0560 & 4.1 \\ \mathrm{H}(69) & 0.1978 & 0.1370 & -0.1442 & 6.0 \\ \mathrm{H}(70) & 0.2037 & 0.2988 & -0.1254 & 4.0 \\ \mathrm{H}(71) & 0.2626 & 0.3632 & -0.0204 & 5.3 \\ \mathrm{H}(72) & 0.1799 & 0.3195 & 0.1108 & 5.0 \\ \mathrm{H}(73) & 0.0959 & 0.4625 & 0.1139 & 6.5 \\ \mathrm{H}(74) & 0.1696 & 0.6086 & 0.1136 & 5.1 \\ \mathrm{H}(75) & 0.3161 & 0.6190 & 0.1038 & 4.8 \\ \mathrm{H}(76) & 0.4040 & 0.4801 & 0.1067 & \mathrm{~T}\end{array}$

a Estimated standard deviations are given in parentheses. ${ }^{\mathrm{b}} \mathrm{The} \mathrm{Pt}, \mathrm{Cl}$, and $\mathrm{P}$ atoms were assigned with anisotropic thermal parameters given as the isotropic equivalent displacement parameter defined as $B_{\text {eq }}=\left(8 \pi^{2} / 3\right)\left(U_{11}\left(a a^{*}\right)^{2}+U_{22}\left(b b^{*}\right)^{2}+U_{33}\left(c c^{*}\right)^{2}+2 U_{12} a a^{*} b b^{*} \cos \gamma+2 U_{13} a a^{*} c c^{*} \cos \beta+\right.$ $2 U_{23} b b^{*} c c^{*} \cos \alpha$ ). Other non-hydrogen atoms were refined isotropically. ${ }^{c}$ All hydrogen atoms except for solvents were calculated with $\mathrm{C}-\mathrm{H}=0.95 \AA$, were not refined with the appropriate $B$ iso. $\mathrm{d}$ Occupancy $=0.5$. e Occupancy $=0.3 .{ }^{\mathrm{f}}$ Occupancy $=0.7$.

Table S2-3. Final Anisotropic Thermal Parameters for 3.2.5 $\mathrm{CH}_{2} \mathrm{Cl}_{2}{ }^{\mathrm{a}, \mathrm{b}}$

\begin{tabular}{llllclr}
\hline atom & $U_{11}$ & $U_{22}$ & $U_{33}$ & $U_{12}$ & $U_{13}$ & $U_{23}$ \\
\hline $\mathrm{Pt}(1)$ & $0.0297(6)$ & $0.0401(6)$ & $0.0220(5)$ & $-0.0075(5)$ & $0.0066(4)$ & $-0.0003(4)$ \\
$\mathrm{Pt}(2)$ & $0.0283(5)$ & $0.0314(5)$ & $0.0218(5)$ & $-0.0048(4)$ & $0.0073(4)$ & $0.0028(4)$ \\
$\mathrm{Pt}(3)$ & $0.0287(4)$ & $0.0280(5)$ & $0.0219(4)$ & $-0.0032(4)$ & $0.0080(3)$ & $0.0006(4)$ \\
$\mathrm{Cl}(1)$ & $0.079(7)$ & $0.24(2)$ & $0.16(1)$ & $0.015(9)$ & $0.041(8)$ & $0.10(1)$ \\
$\mathrm{Cl}(2)$ & $0.11(1)$ & $0.20(2)$ & $0.27(2)$ & $-0.03(1)$ & $0.06(1)$ & $0.01(2)$ \\
$\mathrm{Cl}(3)$ & $0.26(2)$ & $0.16(2)$ & $0.20(2)$ & $0.06(1)$ & $0.14(2)$ & $0.07(1)$ \\
$\mathrm{Cl}(4)$ & $0.107(9)$ & $0.23(2)$ & $0.25(2)$ & $0.01(1)$ & $0.05(1)$ & $0.15(2)$ \\
$\mathrm{Cl}(5)$ & $0.32(4)$ & $0.27(4)$ & $0.14(2)$ & $-0.26(4)$ & $-0.13(3)$ & $0.11(2)$ \\
$\mathrm{Cl}(6)$ & $0.05(1)$ & $0.28(4)$ & $0.09(1)$ & $0.00(2)$ & $0.03(1)$ & $-0.03(2)$ \\
$\mathrm{P}(1)$ & $0.029(3)$ & $0.044(4)$ & $0.020(3)$ & $0.000(3)$ & $0.005(3)$ & $0.001(3)$ \\
$\mathrm{P}(2)$ & $0.035(4)$ & $0.026(4)$ & $0.022(3)$ & $-0.001(3)$ & $0.009(2)$ & $0.004(3)$ \\
$\mathrm{P}(3)$ & $0.033(4)$ & $0.033(4)$ & $0.024(3)$ & $-0.008(3)$ & $0.010(3)$ & $0.003(3)$ \\
$\mathrm{P}(4)$ & $0.034(4)$ & $0.032(4)$ & $0.032(3)$ & $-0.004(3)$ & $0.019(3)$ & $0.002(3)$ \\
$\mathrm{P}(5)$ & $0.033(3)$ & $0.035(4)$ & $0.025(3)$ & $-0.008(3)$ & $0.016(3)$ & $-0.001(3)$ \\
$\mathrm{P}(6)$ & $0.035(4)$ & $0.028(4)$ & $0.028(3)$ & $-0.001(3)$ & $0.008(3)$ & $0.010(2)$ \\
$\mathrm{P}(11)$ & $0.096(6)$ & $0.054(5)$ & $0.034(4)$ & $0.008(5)$ & $0.015(4)$ & $0.002(4)$ \\
$\mathrm{P}(12)$ & $0.072(5)$ & $0.046(5)$ & $0.030(4)$ & $0.005(4)$ & $0.023(4)$ & $-0.005(3)$ \\
\hline
\end{tabular}

a Estmated standard deviations are given in parentheses. $b$ Parameters have the form exp($\left.2 \pi^{2}\left(U_{11} h^{2} a^{* 2}+\cdots+2 U_{12} h k a^{*} b^{*} \cos \gamma+\cdots\right)\right)$

Table S2-4. Bond Lengths for Non-hydrogen Atoms of 3.2.5 $\mathrm{CH}_{2} \mathrm{Cl}_{2}{ }^{\mathrm{a}}$

\begin{tabular}{llllll}
\hline atom & atom & distance & atom & atom & distance \\
\hline $\mathrm{Pt}(1)$ & $\mathrm{Pt}(3)$ & $2.728(2)$ & $\mathrm{P}(3)$ & $\mathrm{C}(321)$ & $1.85(3)$ \\
$\mathrm{Pt}(1)$ & $\mathrm{P}(1)$ & $2.273(8)$ & $\mathrm{P}(4)$ & $\mathrm{C}(5)$ & $1.81(3)$ \\
$\mathrm{Pt}(1)$ & $\mathrm{P}(4)$ & $2.291(8)$ & $\mathrm{P}(4)$ & $\mathrm{C}(411)$ & $1.84(3)$ \\
$\mathrm{Pt}(1)$ & $\mathrm{C}(2)$ & $1.85(3)$ & $\mathrm{P}(4)$ & $\mathrm{C}(421)$ & $1.88(3)$ \\
$\mathrm{Pt}(2)$ & $\mathrm{Pt}(3)$ & $2.696(2)$ & $\mathrm{P}(5)$ & $\mathrm{C}(5)$ & $1.80(3)$
\end{tabular}




\begin{tabular}{|c|c|c|c|c|c|}
\hline $\operatorname{Pt}(2)$ & $\mathrm{P}(3)$ & $2.283(7)$ & $\mathrm{P}(5)$ & $C(6)$ & $1.84(3)$ \\
\hline $\operatorname{Pt}(2)$ & $P(6)$ & $2.287(8)$ & $P(5)$ & $C(511)$ & $1.81(3)$ \\
\hline $\operatorname{Pt}(2)$ & $C(1)$ & $1.94(3)$ & $\mathrm{P}(6)$ & $C(6)$ & $1.83(3)$ \\
\hline $\mathrm{Pt}(3)$ & $P(2)$ & $2.267(7)$ & $\mathrm{P}(6)$ & $C(611)$ & $1.80(2)$ \\
\hline $\mathrm{Pt}(3)$ & $P(5)$ & $2.245(7)$ & $P(6)$ & $C(621)$ & $1.80(3)$ \\
\hline $\mathrm{Cl}(1)$ & $C(701)$ & $1.91(5)$ & $\mathrm{P}(11)$ & $\mathrm{F}(11)$ & $1.61(2)$ \\
\hline $\mathrm{Cl}(2)$ & $C(701)$ & $1.65(5)$ & $\mathrm{P}(11)$ & $\mathrm{F}(12)$ & $1.60(2)$ \\
\hline $\mathrm{Cl}(3)$ & $C(702)$ & $1.6(1)$ & $P(11)$ & $\mathrm{F}(13)$ & $1.60(2)$ \\
\hline $\mathrm{Cl}(3)$ & $C(703)$ & $1.72(5)$ & $\mathrm{P}(11)$ & $\mathrm{F}(14)$ & $1.57(2)$ \\
\hline $\mathrm{Cl}(4)$ & $C(702)$ & $2.1(2)$ & $\mathrm{P}(11)$ & $\mathrm{F}(15)$ & $1.58(2)$ \\
\hline $\mathrm{Cl}(4)$ & $C(703)$ & $1.73(5)$ & $P(11)$ & $F(16)$ & $1.60(2)$ \\
\hline $\mathrm{Cl}(5)$ & $C(704)$ & $1.8(1)$ & $\mathrm{P}(12)$ & $\mathrm{F}(21)$ & $1.57(3)$ \\
\hline $\mathrm{Cl}(6)$ & $C(704)^{*}$ & $1.3(1)$ & $P(12)$ & $\mathrm{F}(22)$ & $1.57(3)$ \\
\hline $\mathrm{P}(1)$ & $C(3)$ & $1.82(3)$ & $\mathrm{P}(12)$ & $F(23)$ & $1.60(4)$ \\
\hline $\mathrm{P}(1)$ & $C(111)$ & $1.85(3)$ & $\mathrm{P}(12)$ & $\mathrm{F}(24)$ & $1.57(3)$ \\
\hline $\mathrm{P}(1)$ & $C(121)$ & $1.79(3)$ & $P(12)$ & $F(25)$ & $1.56(3)$ \\
\hline $\mathrm{P}(2)$ & $C(3)$ & $1.80(3)$ & $\mathrm{P}(12)$ & $F(26)$ & $1.52(5)$ \\
\hline $\mathrm{P}(2)$ & $C(4)$ & $1.83(2)$ & $\mathrm{N}(1)$ & $C(1)$ & $1.22(4)$ \\
\hline $\mathrm{P}(2)$ & $C(211)$ & $1.78(3)$ & $\mathrm{N}(1)$ & $C(11)$ & $1.46(5)$ \\
\hline $\mathrm{P}(3)$ & $C(4)$ & $1.83(2)$ & $\mathrm{N}(2)$ & $C(2)$ & $1.29(3)$ \\
\hline $\mathrm{P}(3)$ & $C(311)$ & 1.83(3) & $\mathrm{N}(2)$ & $C(21)$ & $1.45(4)$ \\
\hline$C(11)$ & $C(12)$ & $1.65(7)$ & $C(213)$ & $C(214)$ & $1.36(4)$ \\
\hline$C(11)$ & $C(13)$ & $1.42(5)$ & $C(214)$ & $C(215)$ & $1.37(4)$ \\
\hline$C(11)$ & $C(14)$ & 1.61(7) & $C(215)$ & $C(216)$ & $1.35(4)$ \\
\hline$C(21)$ & $C(22)$ & 1.41(5) & $C(311)$ & $C(312)$ & $1.37(4)$ \\
\hline$C(21)$ & $C(26)$ & $1.23(5)$ & $C(311)$ & $C(316)$ & $1.37(4)$ \\
\hline$C(22)$ & $C(23)$ & $1.53(5)$ & $C(312)$ & $C(313)$ & $1.35(4)$ \\
\hline$C(22)$ & $C(27)$ & $1.45(6)$ & $C(313)$ & $C(314)$ & $1.39(5)$ \\
\hline$C(23)$ & $C(24)$ & $1.37(5)$ & $C(314)$ & $C(315)$ & $1.35(4)$ \\
\hline$C(24)$ & $C(25)$ & $1.33(5)$ & $C(315)$ & $C(316)$ & $1.41(4)$ \\
\hline$C(25)$ & $C(26)$ & 1.33(5) & $C(321)$ & $C(322)$ & $1.39(5)$ \\
\hline$C(26)$ & $C(28)$ & $1.55(6)$ & $C(321)$ & $C(326)$ & $1.40(4)$ \\
\hline$C(111)$ & $C(112)$ & $1.35(4)$ & $C(322)$ & $C(323)$ & $1.41(6)$ \\
\hline$C(111)$ & $C(116)$ & 1.31(5) & $C(323)$ & $C(324)$ & $1.33(6)$ \\
\hline$C(112)$ & $C(113)$ & $1.37(4)$ & $C(324)$ & $C(325)$ & $1.40(5)$ \\
\hline$C(113)$ & $C(114)$ & $1.33(5)$ & $C(325)$ & $C(326)$ & $1.43(4)$ \\
\hline$C(114)$ & $C(115)$ & $1.47(6)$ & $C(411)$ & $C(412)$ & $1.36(4)$ \\
\hline$C(115)$ & $C(116)$ & 1.42(5) & $C(411)$ & $C(416)$ & $1.36(3)$ \\
\hline$C(121)$ & $C(122)$ & $1.45(4)$ & $C(412)$ & $C(413)$ & $1.44(4)$ \\
\hline$C(121)$ & $C(126)$ & $1.34(4)$ & $C(413)$ & $C(414)$ & $1.34(5)$ \\
\hline$C(122)$ & $C(123)$ & $1.35(4)$ & $C(414)$ & $C(415)$ & $1.42(5)$ \\
\hline$C(123)$ & $C(124)$ & $1.44(4)$ & $C(415)$ & $C(416)$ & $1.40(4)$ \\
\hline$C(124)$ & $C(125)$ & 1.41(5) & $C(421)$ & $C(422)$ & $1.40(4)$ \\
\hline$C(125)$ & $C(126)$ & $1.36(4)$ & $C(421)$ & $C(426)$ & $1.35(4)$ \\
\hline$C(211)$ & $C(212)$ & $1.34(3)$ & $C(422)$ & $C(423)$ & $1.39(4)$ \\
\hline$C(211)$ & $C(216)$ & $1.36(4)$ & $C(423)$ & $C(424)$ & $1.35(4)$ \\
\hline$C(212)$ & $C(213)$ & $1.51(4)$ & $C(424)$ & $C(425)$ & $1.37(4)$ \\
\hline$C(425)$ & $C(426)$ & $1.39(4)$ & & & \\
\hline$C(511)$ & $C(512)$ & $1.43(4)$ & & & \\
\hline$C(511)$ & $C(516)$ & $1.41(4)$ & & & \\
\hline$C(512)$ & C(513) & $1.38(4)$ & & & \\
\hline$C(513)$ & $C(514)$ & $1.34(4)$ & & & \\
\hline$C(514)$ & $C(515)$ & $1.48(5)$ & & & \\
\hline$C(515)$ & $C(516)$ & $1.29(4)$ & & & \\
\hline$C(611)$ & $C(612)$ & $1.38(4)$ & & & \\
\hline$C(611)$ & $C(616)$ & $1.42(4)$ & & & \\
\hline
\end{tabular}




$\begin{array}{lll}\mathrm{C}(612) & \mathrm{C}(613) & 1.42(4) \\ \mathrm{C}(613) & \mathrm{C}(614) & 1.32(5) \\ \mathrm{C}(614) & \mathrm{C}(615) & 1.35(5) \\ \mathrm{C}(615) & \mathrm{C}(616) & 1.35(4) \\ \mathrm{C}(621) & \mathrm{C}(622) & 1.42(4) \\ \mathrm{C}(621) & \mathrm{C}(626) & 1.39(4) \\ \mathrm{C}(622) & \mathrm{C}(623) & 1.38(4) \\ \mathrm{C}(623) & \mathrm{C}(624) & 1.36(4) \\ \mathrm{C}(624) & \mathrm{C}(625) & 1.32(5) \\ \mathrm{C}(625) & \mathrm{C}(626) & 1.36(4)\end{array}$

a Estimated standard deviations are given in parentheses.

Table S2-5. Bond Angles for Non-hydrogen Atoms of 3.2.5 $\mathrm{CH}_{2} \mathrm{Cl}_{2}{ }^{\mathrm{a}}$

\begin{tabular}{|c|c|c|c|c|c|c|c|}
\hline atom & atom & atom & angle & atom & atom & atom & angle \\
\hline$\overline{\operatorname{Pt}(3)}$ & $\operatorname{Pt}(1)$ & $\mathrm{P}(1)$ & $82.4(2)$ & $\operatorname{Pt}(3)$ & $\mathrm{P}(2)$ & $C(3)$ & $113.4(9)$ \\
\hline $\operatorname{Pt}(3)$ & $\operatorname{Pt}(1)$ & $\mathrm{P}(4)$ & $85.7(2)$ & $\operatorname{Pt}(3)$ & $\mathrm{P}(2)$ & $C(4)$ & $110.4(8)$ \\
\hline $\operatorname{Pt}(3)$ & $\operatorname{Pt}(1)$ & $C(2)$ & $175.6(9)$ & $\operatorname{Pt}(3)$ & $\mathrm{P}(2)$ & $C(211)$ & 118.1(9) \\
\hline $\mathrm{P}(1)$ & $\operatorname{Pt}(1)$ & $P(4)$ & 168.1(3) & $C(3)$ & $P(2)$ & $C(4)$ & $102(1)$ \\
\hline $\mathrm{P}(1)$ & $\operatorname{Pt}(1)$ & $C(2)$ & $97.6(9)$ & $C(3)$ & $\mathrm{P}(2)$ & $C(211)$ & $105(1)$ \\
\hline $\mathrm{P}(4)$ & $\operatorname{Pt}(1)$ & $C(2)$ & $94.2(9)$ & $C(4)$ & $P(2)$ & $C(211)$ & $106(1)$ \\
\hline $\operatorname{Pt}(3)$ & $\operatorname{Pt}(2)$ & $P(3)$ & $90.1(2)$ & $\operatorname{Pt}(2)$ & $\mathrm{P}(3)$ & $C(4)$ & $116.4(8)$ \\
\hline $\operatorname{Pt}(3)$ & $\operatorname{Pt}(2)$ & $P(6)$ & $87.8(2)$ & $\operatorname{Pt}(2)$ & $\mathrm{P}(3)$ & $C(311)$ & 117.1(9) \\
\hline $\operatorname{Pt}(3)$ & $\operatorname{Pt}(2)$ & $C(1)$ & $177.0(8)$ & $\operatorname{Pt}(2)$ & $\mathrm{P}(3)$ & $C(321)$ & 108.2(9) \\
\hline $\mathrm{P}(3)$ & $\operatorname{Pt}(2)$ & $P(6)$ & $170.9(3)$ & $C(4)$ & $\mathrm{P}(3)$ & $C(311)$ & 104(1) \\
\hline $\mathrm{P}(3)$ & $\operatorname{Pt}(2)$ & $C(1)$ & $92.9(8)$ & $C(4)$ & $P(3)$ & $C(321)$ & 105(1) \\
\hline$P(6)$ & $\operatorname{Pt}(2)$ & $C(1)$ & $89.4(9)$ & $C(311)$ & $\mathrm{P}(3)$ & $C(321)$ & $106(1)$ \\
\hline $\operatorname{Pt}(1)$ & $\operatorname{Pt}(3)$ & $\operatorname{Pt}(2)$ & $176.39(6)$ & $\operatorname{Pt}(1)$ & $\mathrm{P}(4)$ & $C(5)$ & $109(1)$ \\
\hline $\operatorname{Pt}(1)$ & $\operatorname{Pt}(3)$ & $P(2)$ & $93.6(2)$ & $\operatorname{Pt}(1)$ & $\mathrm{P}(4)$ & $C(411)$ & $120.5(9)$ \\
\hline $\operatorname{Pt}(1)$ & $\operatorname{Pt}(3)$ & $P(5)$ & $92.3(2)$ & $\operatorname{Pt}(1)$ & $P(4)$ & $C(421)$ & $116.2(8)$ \\
\hline $\operatorname{Pt}(2)$ & $\operatorname{Pt}(3)$ & $P(2)$ & $88.0(2)$ & $C(5)$ & $\mathrm{P}(4)$ & $C(411)$ & $104(1)$ \\
\hline $\operatorname{Pt}(2)$ & $\operatorname{Pt}(3)$ & $P(5)$ & $86.2(2)$ & $C(5)$ & $\mathrm{P}(4)$ & $C(421)$ & $108(1)$ \\
\hline$P(2)$ & $\operatorname{Pt}(3)$ & $P(5)$ & 173.1(3) & $C(411)$ & $\mathrm{P}(4)$ & $C(421)$ & 97(1) \\
\hline$C(702)$ & $\mathrm{Cl}(3)$ & $C(703)$ & $81(6)$ & $\operatorname{Pt}(3)$ & $P(5)$ & $C(5)$ & $115.8(9)$ \\
\hline$C(702)$ & $\mathrm{Cl}(4)$ & $C(703)$ & $67(4)$ & $\operatorname{Pt}(3)$ & $P(5)$ & $C(6)$ & $108.6(9)$ \\
\hline $\operatorname{Pt}(1)$ & $\mathrm{P}(1)$ & $C(3)$ & $110(1)$ & $\operatorname{Pt}(3)$ & $P(5)$ & $C(511)$ & $119.0(9)$ \\
\hline $\operatorname{Pt}(1)$ & $\mathrm{P}(1)$ & $C(111)$ & $114.5(9)$ & $C(5)$ & $\mathrm{P}(5)$ & $C(6)$ & $102(1)$ \\
\hline $\operatorname{Pt}(1)$ & $\mathrm{P}(1)$ & $C(121)$ & $116.0(9)$ & $C(5)$ & $P(5)$ & $C(511)$ & 104(1) \\
\hline$C(3)$ & $\mathrm{P}(1)$ & $C(111)$ & $107(1)$ & $C(6)$ & $P(5)$ & $C(511)$ & $106(1)$ \\
\hline$C(3)$ & $\mathrm{P}(1)$ & $C(121)$ & $107(1)$ & $\operatorname{Pt}(2)$ & $P(6)$ & $C(6)$ & $116.7(9)$ \\
\hline$C(111)$ & $\mathrm{P}(1)$ & $C(121)$ & 102(1) & $\operatorname{Pt}(2)$ & $P(6)$ & $C(611)$ & 109.3(8) \\
\hline $\operatorname{Pt}(2)$ & $\mathrm{P}(6)$ & $C(621)$ & $116(1)$ & $\mathrm{F}(22)$ & $\mathrm{P}(12)$ & $\mathrm{F}(25)$ & $88(1)$ \\
\hline$C(6)$ & $P(6)$ & $C(611)$ & 107(1) & $\mathrm{F}(22)$ & $P(12)$ & $F(26)$ & $88(2)$ \\
\hline$C(6)$ & $P(6)$ & $C(621)$ & 102(1) & $\mathrm{F}(23)$ & $\mathrm{P}(12)$ & $\mathrm{F}(24)$ & $90(2)$ \\
\hline$C(611)$ & $P(6)$ & $C(621)$ & 104(1) & $\mathrm{F}(23)$ & $P(12)$ & $\mathrm{F}(25)$ & $81(2)$ \\
\hline $\mathrm{F}(11)$ & $\mathrm{P}(11)$ & $F(12)$ & $89(1)$ & $\mathrm{F}(23)$ & $\mathrm{P}(12)$ & $F(26)$ & $175(2)$ \\
\hline $\mathrm{F}(11)$ & $\mathrm{P}(11)$ & $\mathrm{F}(13)$ & $87(1)$ & $\mathrm{F}(24)$ & $P(12)$ & $\mathrm{F}(25)$ & $92(1)$ \\
\hline $\mathrm{F}(11)$ & $\mathrm{P}(11)$ & $\mathrm{F}(14)$ & $92(1)$ & $\mathrm{F}(24)$ & $P(12)$ & $\mathrm{F}(26)$ & $91(2)$ \\
\hline $\mathrm{F}(11)$ & $\mathrm{P}(11)$ & $F(15)$ & $178(1)$ & $\mathrm{F}(25)$ & $P(12)$ & $F(26)$ & $94(2)$ \\
\hline $\mathrm{F}(11)$ & $P(11)$ & $\mathrm{F}(16)$ & $93(1)$ & $C(1)$ & $\mathrm{N}(1)$ & $C(11)$ & $176(4)$ \\
\hline $\mathrm{F}(12)$ & $P(11)$ & $\mathrm{F}(13)$ & $89(1)$ & $C(2)$ & $\mathrm{N}(2)$ & $C(21)$ & $169(3)$ \\
\hline$F(12)$ & $P(11)$ & $\mathrm{F}(14)$ & $179(1)$ & $\operatorname{Pt}(2)$ & $C(1)$ & $\mathrm{N}(1)$ & $172(3)$ \\
\hline$F(12)$ & $\mathrm{P}(11)$ & $F(15)$ & $89(1)$ & $\operatorname{Pt}(1)$ & $C(2)$ & $\mathrm{N}(2)$ & $165(2)$ \\
\hline$F(12)$ & $\mathrm{P}(11)$ & $F(16)$ & $89(1)$ & $P(1)$ & $C(3)$ & $P(2)$ & $106(1)$ \\
\hline $\mathrm{F}(13)$ & $\mathrm{P}(11)$ & $\mathrm{F}(14)$ & 91(1) & $P(2)$ & $C(4)$ & $P(3)$ & 109(1) \\
\hline$F(13)$ & $\mathrm{P}(11)$ & $\mathrm{F}(15)$ & $93(1)$ & $\mathrm{P}(4)$ & $C(5)$ & $P(5)$ & $108(1)$ \\
\hline
\end{tabular}




\begin{tabular}{|c|c|c|c|c|c|c|c|}
\hline $\mathrm{F}(13)$ & $\mathrm{P}(11)$ & $\mathrm{F}(16)$ & $178(1)$ & $\mathrm{P}(5)$ & $C(6)$ & $P(6)$ & $106(1)$ \\
\hline $\mathrm{F}(14)$ & $\mathrm{P}(11)$ & $\mathrm{F}(15)$ & $90(1)$ & $\mathrm{N}(1)$ & $C(11)$ & $C(12)$ & 103(3) \\
\hline $\mathrm{F}(14)$ & $\mathrm{P}(11)$ & $\mathrm{F}(16)$ & $91(1)$ & $\mathrm{N}(1)$ & $C(11)$ & $C(13)$ & $110(4)$ \\
\hline $\mathrm{F}(15)$ & $\mathrm{P}(11)$ & $\mathrm{F}(16)$ & $87(1)$ & $\mathrm{N}(1)$ & $C(11)$ & $C(14)$ & $103(4)$ \\
\hline $\mathrm{F}(21)$ & $\mathrm{P}(12)$ & $\mathrm{F}(22)$ & $90(1)$ & $C(12)$ & $C(11)$ & $C(13)$ & 101(4) \\
\hline $\mathrm{F}(21)$ & $\mathrm{P}(12)$ & $\mathrm{F}(23)$ & $93(2)$ & $C(12)$ & $C(11)$ & $C(14)$ & $134(5)$ \\
\hline $\mathrm{F}(21)$ & $\mathrm{P}(12)$ & $\mathrm{F}(24)$ & $90(1)$ & $C(13)$ & $C(11)$ & $C(14)$ & $104(4)$ \\
\hline $\mathrm{F}(21)$ & $\mathrm{P}(12)$ & $F(25)$ & $174(2)$ & $\mathrm{N}(2)$ & $C(21)$ & $C(22)$ & 109(3) \\
\hline $\mathrm{F}(21)$ & $\mathrm{P}(12)$ & $\mathrm{F}(26)$ & $92(2)$ & $\mathrm{N}(2)$ & $C(21)$ & $C(26)$ & $122(4)$ \\
\hline $\mathrm{F}(22)$ & $\mathrm{P}(12)$ & $\mathrm{F}(23)$ & $92(2)$ & $C(22)$ & $C(21)$ & $C(26)$ & $128(4)$ \\
\hline $\mathrm{F}(22)$ & $\mathrm{P}(12)$ & $\mathrm{F}(24)$ & 179(1) & $C(21)$ & $C(22)$ & $C(23)$ & $111(4)$ \\
\hline$C(21)$ & $C(22)$ & $C(27)$ & $132(4)$ & $C(212)$ & $C(211)$ & $C(216)$ & $118(3)$ \\
\hline$C(23)$ & $C(22)$ & $C(27)$ & $117(4)$ & $C(211)$ & $C(212)$ & $C(213)$ & $122(3)$ \\
\hline$C(22)$ & $C(23)$ & $C(24)$ & $119(4)$ & $C(212)$ & $C(213)$ & $C(214)$ & $112(3)$ \\
\hline$C(23)$ & $C(24)$ & $C(25)$ & $116(4)$ & $C(213)$ & $C(214)$ & $C(215)$ & $126(3)$ \\
\hline$C(24)$ & $C(25)$ & $C(26)$ & $128(5)$ & $C(214)$ & $C(215)$ & $C(216)$ & $117(3)$ \\
\hline$C(21)$ & $C(26)$ & $C(25)$ & $117(4)$ & $C(211)$ & $C(216)$ & $C(215)$ & $124(3)$ \\
\hline$C(21)$ & $C(26)$ & $C(28)$ & $124(4)$ & $P(3)$ & $C(311)$ & $C(312)$ & $124(2)$ \\
\hline$C(25)$ & $C(26)$ & $C(28)$ & $119(4)$ & $P(3)$ & $C(311)$ & $C(316)$ & $117(2)$ \\
\hline $\mathrm{P}(1)$ & $C(111)$ & $C(112)$ & $119(2)$ & $C(312)$ & $C(311)$ & $C(316)$ & $119(3)$ \\
\hline $\mathrm{P}(1)$ & $C(111)$ & $C(116)$ & $120(2)$ & $C(311)$ & $C(312)$ & $C(313)$ & $123(3)$ \\
\hline$C(112)$ & $C(111)$ & $C(116)$ & 121(3) & $C(312)$ & $C(313)$ & $C(314)$ & $118(3)$ \\
\hline$C(111)$ & $C(112)$ & $C(113)$ & $123(3)$ & $C(313)$ & $C(314)$ & $C(315)$ & $122(3)$ \\
\hline$C(112)$ & $C(113)$ & $\mathrm{C}(114)$ & $120(3)$ & $C(314)$ & $C(315)$ & $C(316)$ & $119(3)$ \\
\hline$C(113)$ & $C(114)$ & $C(115)$ & $118(4)$ & $C(311)$ & $C(316)$ & $C(315)$ & $120(3)$ \\
\hline$C(114)$ & $C(115)$ & $C(116)$ & $118(5)$ & $\mathrm{P}(3)$ & $C(321)$ & $C(322)$ & $124(3)$ \\
\hline$C(111)$ & $C(116)$ & $C(115)$ & $119(4)$ & $P(3)$ & $C(321)$ & $C(326)$ & $117(2)$ \\
\hline $\mathrm{P}(1)$ & $C(121)$ & $C(122)$ & $122(2)$ & $C(322)$ & $C(321)$ & $C(326)$ & $119(3)$ \\
\hline $\mathrm{P}(1)$ & $C(121)$ & $C(126)$ & $123(2)$ & $C(321)$ & $C(322)$ & $C(323)$ & $123(4)$ \\
\hline$C(122)$ & $C(121)$ & $C(126)$ & 115(3) & $C(322)$ & $C(323)$ & $C(324)$ & $118(5)$ \\
\hline$C(121)$ & $C(122)$ & $C(123)$ & 122(3) & $C(323)$ & $C(324)$ & $C(325)$ & $122(4)$ \\
\hline$C(122)$ & $C(123)$ & $C(124)$ & $120(3)$ & $C(324)$ & $C(325)$ & $C(326)$ & $120(4)$ \\
\hline$C(123)$ & $C(124)$ & $C(125)$ & 118(3) & $C(321)$ & $C(326)$ & $C(325)$ & $118(3)$ \\
\hline$C(124)$ & $C(125)$ & $C(126)$ & 119(3) & $\mathrm{P}(4)$ & $C(411)$ & $C(412)$ & $118(2)$ \\
\hline$C(121)$ & $C(126)$ & $C(125)$ & 126(3) & $\mathrm{P}(4)$ & $C(411)$ & $C(416)$ & $124(2)$ \\
\hline $\mathrm{P}(2)$ & $C(211)$ & $C(212)$ & $120(2)$ & $C(412)$ & $C(411)$ & $C(416)$ & $117(3)$ \\
\hline $\mathrm{P}(2)$ & $C(211)$ & $C(216)$ & $122(2)$ & $C(411)$ & $C(412)$ & $C(413)$ & $120(3)$ \\
\hline$C(412)$ & $C(413)$ & $\mathrm{C}(414)$ & $122(3)$ & $C(614)$ & $C(615)$ & $C(616)$ & $123(3)$ \\
\hline$C(413)$ & $C(414)$ & $C(415)$ & $117(4)$ & $C(611)$ & $C(616)$ & $C(615)$ & $117(3)$ \\
\hline$C(414)$ & $C(415)$ & $C(416)$ & 119(3) & $P(6)$ & $C(621)$ & $C(622)$ & $120(2)$ \\
\hline$C(411)$ & $C(416)$ & $C(415)$ & $123(3)$ & $P(6)$ & $C(621)$ & $C(626)$ & $123(2)$ \\
\hline $\mathrm{P}(4)$ & $C(421)$ & $C(422)$ & $115(2)$ & $C(622)$ & $C(621)$ & $C(626)$ & $117(3)$ \\
\hline $\mathrm{P}(4)$ & $C(421)$ & $C(426)$ & $122(2)$ & $C(621)$ & $C(622)$ & $C(623)$ & $119(3)$ \\
\hline$C(422)$ & $C(421)$ & $C(426)$ & 123(3) & $C(622)$ & $C(623)$ & $C(624)$ & $121(3)$ \\
\hline$C(421)$ & $C(422)$ & $C(423)$ & 116(3) & $C(623)$ & $C(624)$ & $C(625)$ & $119(3)$ \\
\hline$C(422)$ & $C(423)$ & $C(424)$ & 123(3) & $C(624)$ & $C(625)$ & $C(626)$ & $123(3)$ \\
\hline$C(423)$ & $C(424)$ & $C(425)$ & 118(3) & $C(621)$ & $C(626)$ & $C(625)$ & $120(3)$ \\
\hline$C(424)$ & $C(425)$ & $C(426)$ & $122(3)$ & $\mathrm{Cl}(1)$ & $C(701)$ & $\mathrm{Cl}(2)$ & $107(3)$ \\
\hline$C(421)$ & $C(426)$ & $C(425)$ & 117(3) & $\mathrm{Cl}(3)$ & $C(702)$ & $\mathrm{Cl}(4)$ & $100(8)$ \\
\hline$P(5)$ & $C(511)$ & $C(512)$ & $120(2)$ & $\mathrm{Cl}(3)$ & $C(703)$ & $\mathrm{Cl}(4)$ & $112(3)$ \\
\hline$P(5)$ & $C(511)$ & $C(516)$ & $124(2)$ & $\mathrm{Cl}(5)$ & $C(704)$ & $\mathrm{Cl}(6)$ & $101(7)$ \\
\hline$C(512)$ & $C(511)$ & $C(516)$ & 115(3) & & & & \\
\hline$C(511)$ & $C(512)$ & $C(513)$ & 122(3) & & & & \\
\hline$C(512)$ & $C(513)$ & $C(514)$ & 121(3) & & & & \\
\hline$C(513)$ & $C(514)$ & $C(515)$ & 116(3) & & & & \\
\hline$C(514)$ & $C(515)$ & $C(516)$ & 123(3) & & & & \\
\hline
\end{tabular}


$\mathrm{C}(511) \quad \mathrm{C}(516) \quad \mathrm{C}(515) \quad 122(3)$

$\mathrm{P}(6) \quad \mathrm{C}(611) \quad \mathrm{C}(612) \quad 118(2)$

$\mathrm{P}(6) \quad \mathrm{C}(611) \quad \mathrm{C}(616) \quad 123(2)$

$\mathrm{C}(612) \quad \mathrm{C}(611) \quad \mathrm{C}(616) \quad 119(2)$

$\begin{array}{llll}C(611) & C(612) & C(613) & 122(3)\end{array}$

$\mathrm{C}(612) \quad \mathrm{C}(613) \quad \mathrm{C}(614) \quad 116(3)$

$\begin{array}{llll}\mathrm{C}(613) & \mathrm{C}(614) & \mathrm{C}(615) & 123(4)\end{array}$

a Estimated deviations are given in parentheses. 
Table S3-1. Crystallographic and Experimental Data for 4

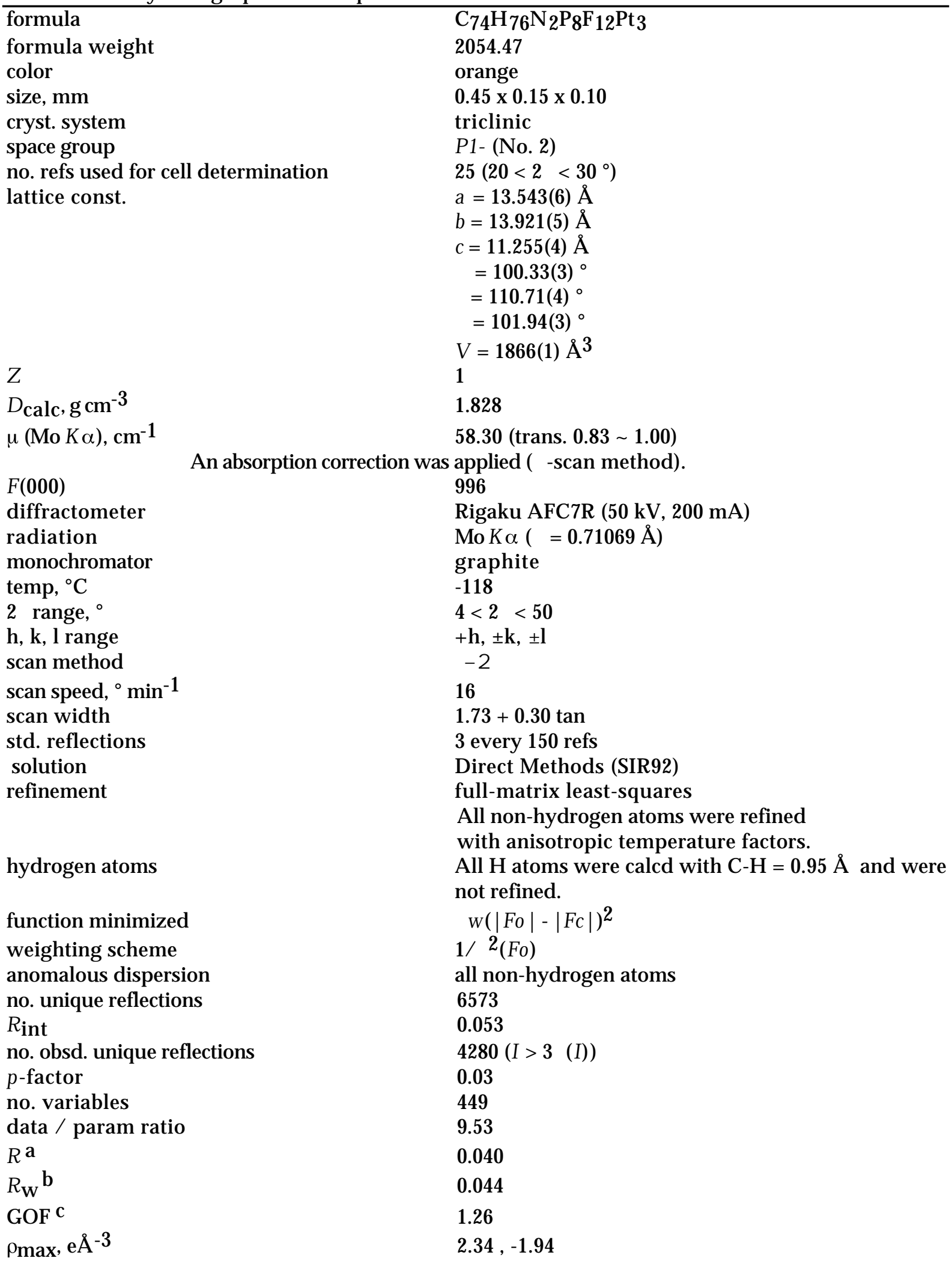

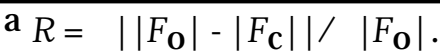

$\mathrm{b}_{R_{\mathrm{W}}}=\left[\Sigma w\left(\left|F_{\mathrm{O}}\right|-\left|F_{\mathrm{C}}\right|\right)^{2} / \Sigma w\left|F_{\mathrm{O}}\right|^{2}\right]^{1 / 2}\left(w=1 / \sigma^{2}\left(F_{\mathrm{O}}\right)\right)$.

${ }^{c} \mathrm{GOF}=\left[\Sigma w\left(\left|F_{\mathrm{O}}\right|-\left|F_{\mathrm{C}}\right|\right)^{2} /\left(N_{\mathrm{O}}-N_{\mathrm{p}}\right]^{1 / 2}\left(N_{\mathrm{O}}=\right.\right.$ no. data, $N_{\mathrm{p}}=$ no. variables $)$.
} 
Table S3-2. Final Positional Parameters and $B_{\text {eq }}$ for $4^{\mathrm{a}-\mathrm{c}}$

\begin{tabular}{|c|c|c|c|c|}
\hline atom & $x$ & $y$ & $z$ & $B_{\mathrm{eq}}$ \\
\hline$\overline{\operatorname{Pt}(1)}$ & $1.21195(4)$ & $0.09883(3)$ & $1.05859(4)$ & $1.67(1)$ \\
\hline $\operatorname{Pt}(2) \mathrm{d}$ & 1.0000 & 0.0000 & 1.0000 & $1.60(1)$ \\
\hline$P(1)$ & $1.2158(2)$ & $0.2059(2)$ & $1.2410(3)$ & $2.04(6)$ \\
\hline $\mathrm{P}(2)$ & $1.0435(2)$ & $0.0234(2)$ & $1.2177(3)$ & $1.58(6)$ \\
\hline$P(3)$ & $1.1911(2)$ & $-0.0110(2)$ & $0.8691(3)$ & $1.79(6)$ \\
\hline $\mathrm{P}(11)$ & $1.1644(3)$ & $0.3378(3)$ & $0.6852(4)$ & $3.79(9)$ \\
\hline $\mathrm{F}(1)$ & $1.2625(8)$ & $0.4179(8)$ & $0.8090(10)$ & $8.2(3)$ \\
\hline $\mathrm{F}(2)$ & $1.0788(8)$ & $0.3860(6)$ & $0.7257(9)$ & $6.0(3)$ \\
\hline $\mathrm{F}(3)$ & $1.176(1)$ & $0.4161(9)$ & $0.601(1)$ & $9.4(4)$ \\
\hline $\mathrm{F}(4)$ & $1.2478(8)$ & $0.2900(8)$ & $0.6437(9)$ & $6.6(3)$ \\
\hline$F(5)$ & $1.0645(8)$ & $0.2588(9)$ & $0.5614(9)$ & $8.2(3)$ \\
\hline$F(6)$ & $1.1547(7)$ & $0.2614(6)$ & $0.7719(8)$ & $5.0(2)$ \\
\hline $\mathrm{N}(1)$ & $1.4355(8)$ & $0.2523(7)$ & $1.112(1)$ & $2.9(2)$ \\
\hline$C(1)$ & $1.3581(9)$ & $0.1890(8)$ & 1.091(1) & $2.1(2)$ \\
\hline$C(2)$ & $1.1192(9)$ & $0.1564(8)$ & $1.3110(10)$ & $1.9(2)$ \\
\hline$C(3)$ & $0.9259(8)$ & $-0.0003(8)$ & $1.2668(9)$ & $1.9(2)$ \\
\hline$C(4)$ & $1.532(1)$ & $0.3434(9)$ & $1.149(1)$ & $3.6(3)$ \\
\hline$C(5)$ & $1.487(1)$ & $0.433(1)$ & $1.161(2)$ & $6.0(5)$ \\
\hline$C(6)$ & $1.575(1)$ & $0.335(1)$ & $1.043(2)$ & $5.0(4)$ \\
\hline$C(7)$ & $1.617(1)$ & $0.343(1)$ & $1.280(2)$ & $5.2(4)$ \\
\hline$C(111)$ & $1.1875(10)$ & $0.3232(8)$ & $1.211(1)$ & $2.6(3)$ \\
\hline$C(112)$ & $1.187(1)$ & $0.3465(9)$ & $1.095(1)$ & $3.4(3)$ \\
\hline$C(113)$ & $1.164(1)$ & $0.434(1)$ & $1.066(1)$ & $5.0(4)$ \\
\hline$C(114)$ & $1.145(1)$ & $0.501(1)$ & $1.159(2)$ & $5.5(5)$ \\
\hline$C(115)$ & $1.149(1)$ & $0.481(1)$ & $1.275(2)$ & $4.7(4)$ \\
\hline$C(116)$ & $1.169(1)$ & $0.3911(9)$ & $1.303(1)$ & $2.8(3)$ \\
\hline$C(121)$ & $1.3496(9)$ & $0.2393(9)$ & $1.378(1)$ & $2.3(3)$ \\
\hline$C(122)$ & $1.404(1)$ & $0.3385(10)$ & $1.466(1)$ & $3.6(3)$ \\
\hline$C(123)$ & $1.508(1)$ & $0.355(1)$ & $1.567(1)$ & $4.8(4)$ \\
\hline$C(124)$ & $1.558(1)$ & $0.280(1)$ & $1.581(1)$ & $4.5(4)$ \\
\hline$C(125)$ & $1.507(1)$ & $0.184(1)$ & $1.493(1)$ & $3.5(3)$ \\
\hline$C(126)$ & $1.404(1)$ & $0.1646(9)$ & $1.395(1)$ & $3.0(3)$ \\
\hline$C(211)$ & $1.1184(9)$ & $-0.0591(8)$ & $1.296(1)$ & $2.2(2)$ \\
\hline$C(212)$ & 1.1997(10) & $-0.0256(9)$ & $1.423(1)$ & $2.7(3)$ \\
\hline$C(213)$ & $1.245(1)$ & $-0.095(1)$ & $1.481(1)$ & $3.5(3)$ \\
\hline$C(214)$ & $1.207(1)$ & $-0.1971(9)$ & $1.414(1)$ & $3.2(3)$ \\
\hline$C(215)$ & $1.125(1)$ & $-0.2303(9)$ & $1.287(1)$ & $3.7(3)$ \\
\hline$C(216)$ & $1.083(1)$ & $-0.1594(8)$ & $1.227(1)$ & $2.9(3)$ \\
\hline$C(311)$ & $1.1653(9)$ & $-0.1459(8)$ & $0.857(1)$ & $2.2(2)$ \\
\hline$C(312)$ & $1.174(1)$ & $-0.180(1)$ & $0.968(1)$ & $3.6(3)$ \\
\hline$C(313)$ & $1.157(1)$ & $-0.283(1)$ & $0.958(2)$ & $5.1(5)$ \\
\hline$C(314)$ & $1.126(1)$ & $-0.3526(10)$ & $0.840(2)$ & $4.4(4)$ \\
\hline$C(315)$ & $1.119(1)$ & $-0.3209(9)$ & $0.730(1)$ & $3.6(3)$ \\
\hline$C(316)$ & $1.1361(9)$ & $-0.2167(9)$ & $0.737(1)$ & $2.9(3)$ \\
\hline$C(321)$ & $1.3081(8)$ & $0.0212(8)$ & $0.824(1)$ & $2.0(2)$ \\
\hline$C(322)$ & $1.3249(10)$ & $0.1038(9)$ & $0.775(1)$ & $2.6(3)$ \\
\hline$C(323)$ & $1.4162(10)$ & $0.1337(9)$ & $0.745(1)$ & $2.8(3)$ \\
\hline$C(324)$ & $1.4933(9)$ & $0.080(1)$ & $0.769(1)$ & $3.1(3)$ \\
\hline$C(325)$ & $1.4784(9)$ & $-0.0029(10)$ & $0.818(1)$ & $2.8(3)$ \\
\hline$C(326)$ & $1.3863(9)$ & $-0.0340(9)$ & $0.845(1)$ & $2.6(3)$ \\
\hline $\mathrm{H}(1)$ & 1.6400 & 0.2685 & 1.2770 & 2.3 \\
\hline $\mathrm{H}(2)$ & 1.5753 & 0.3609 & 1.3560 & 2.3 \\
\hline $\mathrm{H}(3)$ & 1.6878 & 0.4123 & 1.3112 & 2.3 \\
\hline $\mathrm{H}(4)$ & 1.5454 & 0.4944 & 1.1843 & 7.3 \\
\hline $\mathrm{H}(6)$ & 1.5192 & 0.3336 & 0.9622 & 6.2 \\
\hline
\end{tabular}




\begin{tabular}{|c|c|c|c|c|}
\hline $\mathrm{H}(7)$ & 1.5948 & 0.2731 & 1.0326 & 6.2 \\
\hline $\mathrm{H}(8)$ & 0.8894 & -0.0868 & 1.2527 & 2.3 \\
\hline $\mathrm{H}(9)$ & 0.9374 & 0.0455 & 1.3395 & 2.3 \\
\hline $\mathrm{H}(25)$ & 1.1560 & 0.1620 & 1.3996 & 2.3 \\
\hline $\mathrm{H}(26)$ & 1.2019 & 0.3001 & 1.0365 & 3.9 \\
\hline $\mathrm{H}(27)$ & 1.1609 & 0.4483 & 0.9866 & 6.5 \\
\hline $\mathrm{H}(28)$ & 1.1317 & 0.5631 & 1.1447 & 6.4 \\
\hline $\mathrm{H}(29)$ & 1.1353 & 0.5256 & 1.3372 & 5.4 \\
\hline $\mathrm{H}(30)$ & 1.1700 & 0.3766 & 1.3820 & 3.2 \\
\hline $\mathrm{H}(31)$ & 1.3701 & 0.3916 & 1.4591 & 4.2 \\
\hline $\mathrm{H}(32)$ & 1.5452 & 0.4205 & 1.6275 & 5.3 \\
\hline $\mathrm{H}(33)$ & 1.6282 & 0.2947 & 1.6488 & 5.0 \\
\hline $\mathrm{H}(34)$ & 1.5421 & 0.1315 & 1.5016 & 4.3 \\
\hline $\mathrm{H}(35)$ & 1.3685 & 0.0985 & 1.3358 & 3.6 \\
\hline $\mathrm{H}(36)$ & 1.2256 & 0.0443 & 1.4683 & 3.3 \\
\hline $\mathrm{H}(37)$ & 1.3016 & -0.0745 & 1.5664 & 4.1 \\
\hline $\mathrm{H}(38)$ & 1.2356 & -0.2451 & 1.4541 & 3.7 \\
\hline $\mathrm{H}(39)$ & 1.0997 & -0.3004 & 1.2394 & 4.4 \\
\hline $\mathrm{H}(40)$ & 1.0283 & -0.1803 & 1.1376 & 3.3 \\
\hline $\mathrm{H}(41)$ & 1.1947 & -0.1342 & 1.0511 & 4.2 \\
\hline $\mathrm{H}(42)$ & 1.1635 & -0.3060 & 1.0344 & 6.2 \\
\hline $\mathrm{H}(43)$ & 1.1102 & -0.4228 & 0.8372 & 5.5 \\
\hline $\mathrm{H}(44)$ & 1.1040 & -0.3705 & 0.6501 & 4.1 \\
\hline $\mathrm{H}(45)$ & 1.1287 & -0.1943 & 0.6612 & 3.2 \\
\hline $\mathrm{H}(46)$ & 1.2726 & 0.1421 & 0.7626 & 3.2 \\
\hline $\mathrm{H}(47)$ & 1.4237 & 0.1901 & 0.7102 & 3.3 \\
\hline $\mathrm{H}(48)$ & 1.5558 & 0.1014 & 0.7499 & 4.0 \\
\hline $\mathrm{H}(49)$ & 1.5323 & -0.0379 & 0.8342 & 3.4 \\
\hline $\mathrm{H}(50)$ & 1.3758 & -0.0912 & 0.8780 & 3.0 \\
\hline $\mathrm{H}(51)$ & 1.6377 & 0.3913 & 1.0676 & 6.2 \\
\hline $\mathrm{H}(52)$ & 1.4298 & 0.4271 & 1.0800 & 7.3 \\
\hline $\mathrm{H}(53)$ & 1.4607 & 0.4353 & 1.2287 & 7.3 \\
\hline $\mathrm{H}(54)$ & 1.0664 & 0.1958 & 1.3005 & 2.3 \\
\hline
\end{tabular}

Table S3-3. Final Anisotropic Thermal Parameters for $4^{\mathrm{a}, \mathrm{b}}$

\begin{tabular}{llllllr}
\hline atom & $U_{11}$ & $U_{22}$ & $U_{33}$ & $U_{12}$ & $U_{13}$ & \multicolumn{1}{c}{$U_{23}$} \\
\hline $\mathrm{Pt}(1)$ & $0.0207(3)$ & $0.0174(2)$ & $0.0221(3)$ & $0.0058(2)$ & $0.0071(2)$ & $0.0013(2)$ \\
$\mathrm{Pt}(2)$ & $0.0197(4)$ & $0.0188(3)$ & $0.0194(3)$ & $0.0063(3)$ & $0.0058(3)$ & $0.0026(3)$ \\
$\mathrm{P}(1)$ & $0.028(2)$ & $0.020(1)$ & $0.025(2)$ & $0.006(1)$ & $0.011(1)$ & $-0.002(1)$ \\
$\mathrm{P}(2)$ & $0.017(1)$ & $0.021(1)$ & $0.020(1)$ & $0.006(1)$ & $0.007(1)$ & $0.002(1)$ \\
$\mathrm{P}(3)$ & $0.022(2)$ & $0.022(1)$ & $0.022(1)$ & $0.007(1)$ & $0.009(1)$ & $0.001(1)$ \\
$\mathrm{P}(11)$ & $0.059(2)$ & $0.038(2)$ & $0.041(2)$ & $0.005(2)$ & $0.023(2)$ & $0.003(2)$ \\
$\mathrm{F}(1)$ & $0.086(8)$ & $0.093(8)$ & $0.082(7)$ & $-0.039(6)$ & $0.035(6)$ & $-0.023(6)$ \\
$\mathrm{F}(2)$ & $0.110(8)$ & $0.060(6)$ & $0.085(7)$ & $0.049(6)$ & $0.056(6)$ & $0.018(5)$ \\
$\mathrm{F}(3)$ & $0.19(1)$ & $0.109(9)$ & $0.112(9)$ & $0.054(9)$ & $0.097(9)$ & $0.074(8)$ \\
$\mathrm{F}(4)$ & $0.076(7)$ & $0.111(8)$ & $0.077(7)$ & $0.035(6)$ & $0.046(6)$ & $0.019(6)$ \\
$\mathrm{F}(5)$ & $0.070(7)$ & $0.14(1)$ & $0.062(6)$ & $0.019(7)$ & $0.014(5)$ & $-0.020(6)$ \\
$\mathrm{F}(6)$ & $0.083(6)$ & $0.049(5)$ & $0.079(6)$ & $0.024(5)$ & $0.049(5)$ & $0.030(5)$ \\
$\mathrm{N}(1)$ & $0.040(7)$ & $0.017(5)$ & $0.050(7)$ & $0.010(5)$ & $0.020(5)$ & $0.000(5)$
\end{tabular}




\begin{tabular}{|c|c|c|c|c|c|c|}
\hline$C(1)$ & $0.014(6)$ & $0.024(6)$ & $0.029(6)$ & $0.009(5)$ & $-0.003(5)$ & $0.001(5)$ \\
\hline$C(2)$ & $0.027(6)$ & $0.018(6)$ & $0.019(6)$ & $0.003(5)$ & $0.007(5)$ & $-0.004(4)$ \\
\hline$C(3)$ & $0.019(6)$ & $0.026(6)$ & $0.015(5)$ & $0.003(5)$ & $0.001(4)$ & $-0.005(4)$ \\
\hline$C(4)$ & $0.041(8)$ & $0.022(7)$ & $0.067(9)$ & $-0.012(6)$ & $0.033(7)$ & $-0.004(6)$ \\
\hline$C(5)$ & $0.06(1)$ & $0.029(8)$ & $0.14(2)$ & $0.004(7)$ & $0.06(1)$ & $0.006(9)$ \\
\hline$C(6)$ & $0.059(10)$ & $0.046(9)$ & $0.07(1)$ & $-0.006(7)$ & $0.038(9)$ & $-0.004(8)$ \\
\hline$C(7)$ & $0.041(9)$ & $0.07(1)$ & $0.07(1)$ & $-0.007(8)$ & $0.018(8)$ & $-0.004(8)$ \\
\hline$C(111)$ & $0.045(8)$ & $0.020(6)$ & $0.038(7)$ & $0.015(5)$ & $0.021(6)$ & $0.003(5)$ \\
\hline$C(112)$ & $0.062(9)$ & $0.032(7)$ & $0.046(8)$ & $0.027(7)$ & $0.025(7)$ & $0.014(6)$ \\
\hline$C(113)$ & $0.12(1)$ & $0.056(10)$ & $0.046(9)$ & $0.054(10)$ & $0.042(9)$ & $0.030(8)$ \\
\hline$C(114)$ & $0.12(1)$ & $0.032(8)$ & $0.10(1)$ & $0.046(9)$ & $0.07(1)$ & $0.033(9)$ \\
\hline$C(115)$ & $0.10(1)$ & $0.030(8)$ & $0.07(1)$ & $0.027(8)$ & $0.058(10)$ & $0.012(7)$ \\
\hline$C(116)$ & $0.044(8)$ & $0.029(7)$ & $0.041(7)$ & $0.018(6)$ & $0.023(6)$ & $0.008(5)$ \\
\hline$C(121)$ & $0.028(7)$ & $0.033(7)$ & $0.023(6)$ & $0.003(5)$ & $0.014(5)$ & $-0.001(5)$ \\
\hline$C(122)$ & $0.035(7)$ & $0.044(8)$ & $0.041(8)$ & $-0.001(6)$ & $0.014(6)$ & $-0.012(6)$ \\
\hline$C(123)$ & $0.043(9)$ & $0.07(1)$ & $0.047(9)$ & $-0.001(8)$ & $0.019(7)$ & $-0.011(8)$ \\
\hline$C(124)$ & $0.032(8)$ & $0.10(1)$ & $0.030(8)$ & $0.015(8)$ & $0.007(6)$ & $0.002(8)$ \\
\hline$C(125)$ & $0.028(7)$ & $0.063(9)$ & $0.050(8)$ & $0.020(7)$ & $0.019(6)$ & $0.021(7)$ \\
\hline$C(126)$ & $0.040(7)$ & $0.036(7)$ & $0.035(7)$ & $0.011(6)$ & $0.017(6)$ & $0.003(6)$ \\
\hline$C(211)$ & $0.030(6)$ & $0.026(6)$ & $0.028(6)$ & $0.010(5)$ & $0.012(5)$ & $0.007(5)$ \\
\hline$C(212)$ & $0.044(8)$ & $0.031(7)$ & $0.026(6)$ & $0.018(6)$ & $0.013(6)$ & $-0.004(5)$ \\
\hline$C(213)$ & $0.045(8)$ & $0.050(8)$ & $0.036(7)$ & $0.025(7)$ & $0.007(6)$ & $0.016(6)$ \\
\hline$C(214)$ & $0.047(8)$ & $0.039(8)$ & $0.043(8)$ & $0.028(6)$ & $0.017(6)$ & $0.019(6)$ \\
\hline$C(215)$ & $0.07(1)$ & $0.026(7)$ & $0.042(8)$ & $0.027(7)$ & $0.014(7)$ & $0.006(6)$ \\
\hline$C(216)$ & $0.049(8)$ & $0.021(6)$ & $0.033(7)$ & $0.016(6)$ & $0.009(6)$ & $0.003(5)$ \\
\hline$C(311)$ & $0.028(6)$ & $0.022(6)$ & $0.027(6)$ & $0.007(5)$ & $0.005(5)$ & $0.001(5)$ \\
\hline$C(312)$ & $0.070(10)$ & $0.044(8)$ & $0.035(7)$ & $0.028(7)$ & $0.024(7)$ & $0.016(6)$ \\
\hline$C(313)$ & $0.11(1)$ & $0.038(9)$ & $0.08(1)$ & $0.038(9)$ & $0.06(1)$ & $0.037(8)$ \\
\hline$C(314)$ & $0.07(1)$ & $0.030(8)$ & $0.08(1)$ & $0.031(7)$ & $0.035(9)$ & $0.014(8)$ \\
\hline$C(315)$ & $0.043(8)$ & $0.030(7)$ & $0.063(9)$ & $0.015(6)$ & $0.027(7)$ & $-0.009(7)$ \\
\hline$C(316)$ & $0.030(7)$ & $0.036(7)$ & $0.040(7)$ & $0.013(6)$ & $0.011(6)$ & $0.005(6)$ \\
\hline$C(321)$ & $0.020(6)$ & $0.033(6)$ & $0.022(6)$ & $0.007(5)$ & $0.010(5)$ & $0.000(5)$ \\
\hline$C(322)$ & $0.036(7)$ & $0.029(7)$ & $0.037(7)$ & $0.013(6)$ & $0.021(6)$ & $0.004(5)$ \\
\hline$C(323)$ & $0.037(7)$ & $0.032(7)$ & $0.032(7)$ & $0.000(6)$ & $0.019(6)$ & $0.002(5)$ \\
\hline$C(324)$ & $0.024(6)$ & $0.065(9)$ & $0.030(7)$ & $0.010(6)$ & $0.017(5)$ & $0.003(6)$ \\
\hline$C(325)$ & $0.029(7)$ & $0.053(8)$ & $0.023(6)$ & $0.020(6)$ & $0.007(5)$ & $0.004(6)$ \\
\hline$C(326)$ & $0.033(7)$ & $0.041(7)$ & $0.025(6)$ & $0.013(6)$ & $0.011(5)$ & $0.007(5)$ \\
\hline
\end{tabular}

a Estmated standard deviations are given in parentheses. ${ }^{b}$ Parameters have the form exp($\left.2 \pi^{2}\left(U_{11} h^{2} a^{* 2}+\cdots+2 U_{12} h k a^{*} b^{*} \cos \gamma+\cdots\right)\right)$.

Table S3-4. Bond Lengths for Non-hydrogen Atoms of $4^{\mathrm{a}}$

\begin{tabular}{llclll}
\hline atom & atom & distance & atom & atom & distance \\
\hline $\mathrm{Pt}(1)$ & $\mathrm{Pt}(2)$ & $2.696(1)$ & $\mathrm{C}(111)$ & $\mathrm{C}(112)$ & $1.40(2)$ \\
$\operatorname{Pt}(1)$ & $\mathrm{P}(1)$ & $2.288(3)$ & $\mathrm{C}(111)$ & $\mathrm{C}(116)$ & $1.39(1)$ \\
$\mathrm{Pt}(1)$ & $\mathrm{P}(3)$ & $2.279(3)$ & $\mathrm{C}(112)$ & $\mathrm{C}(113)$ & $1.38(2)$ \\
$\mathrm{Pt}(1)$ & $\mathrm{C}(1)$ & $1.98(1)$ & $\mathrm{C}(113)$ & $\mathrm{C}(114)$ & $1.40(2)$ \\
$\mathrm{Pt}(2)$ & $\mathrm{P}(2)$ & $2.251(3)$ & $\mathrm{C}(114)$ & $\mathrm{C}(115)$ & $1.37(2)$ \\
$\mathrm{Pt}(2)$ & $\mathrm{P}(2)^{*}$ & $2.251(3)$ & $\mathrm{C}(115)$ & $\mathrm{C}(116)$ & $1.40(2)$ \\
$\mathrm{P}(1)$ & $\mathrm{C}(2)$ & $1.83(1)$ & $\mathrm{C}(121)$ & $\mathrm{C}(122)$ & $1.42(2)$ \\
$\mathrm{P}(1)$ & $\mathrm{C}(111)$ & $1.82(1)$ & $\mathrm{C}(121)$ & $\mathrm{C}(126)$ & $1.40(2)$ \\
$\mathrm{P}(1)$ & $\mathrm{C}(121)$ & $1.82(1)$ & $\mathrm{C}(122)$ & $\mathrm{C}(123)$ & $1.40(2)$ \\
$\mathrm{P}(2)$ & $\mathrm{C}(2)$ & $1.83(1)$ & $\mathrm{C}(123)$ & $\mathrm{C}(124)$ & $1.36(2)$ \\
$\mathrm{P}(2)$ & $\mathrm{C}(3)$ & $1.85(1)$ & $\mathrm{C}(124)$ & $\mathrm{C}(125)$ & $1.37(2)$ \\
$\mathrm{P}(2)$ & $\mathrm{C}(211)$ & $1.83(1)$ & $\mathrm{C}(125)$ & $\mathrm{C}(126)$ & $1.38(2)$ \\
$\mathrm{P}(3)$ & $\mathrm{C}(3)^{*}$ & $1.83(1)$ & $\mathrm{C}(211)$ & $\mathrm{C}(212)$ & $1.38(1)$ \\
$\mathrm{P}(3)$ & $\mathrm{C}(311)$ & $1.81(1)$ & $\mathrm{C}(211)$ & $\mathrm{C}(216)$ & $1.36(1)$
\end{tabular}




\begin{tabular}{llllll}
$\mathrm{P}(3)$ & $\mathrm{C}(321)$ & $1.82(1)$ & $\mathrm{C}(212)$ & $\mathrm{C}(213)$ & $1.39(2)$ \\
$\mathrm{P}(11)$ & $\mathrm{F}(1)$ & $1.572(9)$ & $\mathrm{C}(213)$ & $\mathrm{C}(214)$ & $1.37(2)$ \\
$\mathrm{P}(11)$ & $\mathrm{F}(2)$ & $1.608(9)$ & $\mathrm{C}(214)$ & $\mathrm{C}(215)$ & $1.39(2)$ \\
$\mathrm{P}(11)$ & $\mathrm{F}(3)$ & $1.59(1)$ & $\mathrm{C}(215)$ & $\mathrm{C}(216)$ & $1.40(2)$ \\
$\mathrm{P}(11)$ & $\mathrm{F}(4)$ & $1.584(9)$ & $\mathrm{C}(311)$ & $\mathrm{C}(312)$ & $1.39(2)$ \\
$\mathrm{P}(11)$ & $\mathrm{F}(5)$ & $1.58(1)$ & $\mathrm{C}(311)$ & $\mathrm{C}(316)$ & $1.40(1)$ \\
$\mathrm{P}(11)$ & $\mathrm{F}(6)$ & $1.583(8)$ & $\mathrm{C}(312)$ & $\mathrm{C}(313)$ & $1.38(2)$ \\
$\mathrm{N}(1)$ & $\mathrm{C}(1)$ & $1.14(1)$ & $\mathrm{C}(313)$ & $\mathrm{C}(314)$ & $1.36(2)$ \\
$\mathrm{N}(1)$ & $\mathrm{C}(4)$ & $1.49(1)$ & $\mathrm{C}(314)$ & $\mathrm{C}(315)$ & $1.37(2)$ \\
$\mathrm{C}(4)$ & $\mathrm{C}(5)$ & $1.50(2)$ & $\mathrm{C}(315)$ & $\mathrm{C}(316)$ & $1.40(2)$ \\
$\mathrm{C}(4)$ & $\mathrm{C}(6)$ & $1.50(2)$ & $\mathrm{C}(321)$ & $\mathrm{C}(322)$ & $1.37(2)$ \\
$\mathrm{C}(4)$ & $\mathrm{C}(7)$ & $1.52(2)$ & $\mathrm{C}(321)$ & $\mathrm{C}(326)$ & $1.41(2)$ \\
$\mathrm{C}(322)$ & $\mathrm{C}(323)$ & $1.40(2)$ & & & \\
$\mathrm{C}(323)$ & $\mathrm{C}(324)$ & $1.39(2)$ & & & \\
$\mathrm{C}(324)$ & $\mathrm{C}(325)$ & $1.38(2)$ & & & \\
$\mathrm{C}(325)$ & $\mathrm{C}(326)$ & $1.38(2)$ & & & \\
\hline
\end{tabular}

a Estimated standard deviations are given in parentheses.

Table S3-5. Bond Angles for Non-hydrogen Atoms of $4^{\mathrm{a}}$

\begin{tabular}{|c|c|c|c|c|c|c|c|}
\hline atom & atom & atom & angle & atom & atom & atom & angle \\
\hline$\overline{P t(2)}$ & $\operatorname{Pt}(1)$ & $\mathrm{P}(1)$ & $88.84(8)$ & $\operatorname{Pt}(1)$ & $\mathrm{P}(3)$ & $C(321)$ & $113.4(3)$ \\
\hline $\operatorname{Pt}(2)$ & $\operatorname{Pt}(1)$ & $P(3)$ & 86.31(7) & $C(3)$ & $\mathrm{P}(3)$ & $C(311)$ & 106.1(5) \\
\hline $\operatorname{Pt}(2)$ & $\operatorname{Pt}(1)$ & $C(1)$ & $170.7(3)$ & $C(3)$ & $\mathrm{P}(3)$ & $C(321)$ & $104.9(5)$ \\
\hline$P(1)$ & $\operatorname{Pt}(1)$ & $\mathrm{P}(3)$ & $174.8(1)$ & $C(311)$ & $\mathrm{P}(3)$ & $C(321)$ & $104.6(5)$ \\
\hline $\mathrm{P}(1)$ & $\operatorname{Pt}(1)$ & $C(1)$ & $88.8(3)$ & $\mathrm{F}(1)$ & $\mathrm{P}(11)$ & $\mathrm{F}(2)$ & $89.4(6)$ \\
\hline$P(3)$ & $\operatorname{Pt}(1)$ & $C(1)$ & $95.7(3)$ & $\mathrm{F}(1)$ & $P(11)$ & $F(3)$ & $88.7(6)$ \\
\hline $\operatorname{Pt}(1)$ & $\operatorname{Pt}(2)$ & $\operatorname{Pt}(1)$ & 180.00 & $\mathrm{~F}(1)$ & $\mathrm{P}(11)$ & $\mathrm{F}(4)$ & $91.1(6)$ \\
\hline $\operatorname{Pt}(1)$ & $\operatorname{Pt}(2)$ & $P(2)$ & $88.80(7)$ & $\mathrm{F}(1)$ & $P(11)$ & $F(5)$ & $178.8(7)$ \\
\hline $\operatorname{Pt}(1)$ & $\operatorname{Pt}(2)$ & $P(2)$ & $91.20(7)$ & $\mathrm{F}(1)$ & $\mathrm{P}(11)$ & $F(6)$ & $90.0(6)$ \\
\hline $\operatorname{Pt}(1)$ & $\operatorname{Pt}(2)$ & $\mathrm{P}(2)$ & $91.20(7)$ & $\mathrm{F}(2)$ & $P(11)$ & $F(3)$ & $91.0(6)$ \\
\hline $\operatorname{Pt}(1)$ & $\operatorname{Pt}(2)$ & $P(2)$ & $88.80(7)$ & $\mathrm{F}(2)$ & $P(11)$ & $\mathrm{F}(4)$ & $179.4(6)$ \\
\hline$P(2)$ & $\operatorname{Pt}(2)$ & $P(2)$ & 180.00 & $\mathrm{~F}(2)$ & $P(11)$ & $F(5)$ & $89.4(5)$ \\
\hline $\operatorname{Pt}(1)$ & $\mathrm{P}(1)$ & $C(2)$ & 117.1(3) & $\mathrm{F}(2)$ & $\mathrm{P}(11)$ & $F(6)$ & $89.1(5)$ \\
\hline $\operatorname{Pt}(1)$ & $\mathrm{P}(1)$ & $C(111)$ & $112.5(4)$ & $F(3)$ & $\mathrm{P}(11)$ & $\mathrm{F}(4)$ & $88.8(6)$ \\
\hline $\operatorname{Pt}(1)$ & $\mathrm{P}(1)$ & $C(121)$ & $110.6(4)$ & $\mathrm{F}(3)$ & $\mathrm{P}(11)$ & $F(5)$ & $91.0(7)$ \\
\hline$C(2)$ & $\mathrm{P}(1)$ & $C(111)$ & $104.4(5)$ & $\mathrm{F}(3)$ & $\mathrm{P}(11)$ & $F(6)$ & $178.8(6)$ \\
\hline$C(2)$ & $P(1)$ & $C(121)$ & $103.2(5)$ & $\mathrm{F}(4)$ & $\mathrm{P}(11)$ & $F(5)$ & $90.0(5)$ \\
\hline$C(111)$ & $\mathrm{P}(1)$ & $C(121)$ & $108.2(5)$ & $\mathrm{F}(4)$ & $\mathrm{P}(11)$ & $F(6)$ & $91.2(5)$ \\
\hline $\operatorname{Pt}(2)$ & $\mathrm{P}(2)$ & $C(2)$ & $111.5(4)$ & $\mathrm{F}(5)$ & $\mathrm{P}(11)$ & $F(6)$ & $90.2(6)$ \\
\hline $\operatorname{Pt}(2)$ & $\mathrm{P}(2)$ & $C(3)$ & 116.1(3) & $C(1)$ & $\mathrm{N}(1)$ & $C(4)$ & $173(1)$ \\
\hline $\operatorname{Pt}(2)$ & $P(2)$ & $C(211)$ & $116.7(4)$ & $\operatorname{Pt}(1)$ & $C(1)$ & $\mathrm{N}(1)$ & 170(1) \\
\hline$C(2)$ & $P(2)$ & $C(3)$ & $102.2(5)$ & $\mathrm{P}(1)$ & $C(2)$ & $P(2)$ & $108.7(5)$ \\
\hline$C(2)$ & $P(2)$ & $C(211)$ & $108.8(5)$ & $P(2)$ & $C(3)$ & $\mathrm{P}(3)$ & $105.6(5)$ \\
\hline$C(3)$ & $\mathrm{P}(2)$ & $C(211)$ & $100.1(5)$ & $\mathrm{N}(1)$ & $C(4)$ & $C(5)$ & $105(1)$ \\
\hline $\operatorname{Pt}(1)$ & $\mathrm{P}(3)$ & $C(3)$ & 107.7(3) & $\mathrm{N}(1)$ & $C(4)$ & $C(6)$ & $109(1)$ \\
\hline $\operatorname{Pt}(1)$ & $P(3)$ & $C(311)$ & $119.0(4)$ & $\mathrm{N}(1)$ & $C(4)$ & $C(7)$ & 106(1) \\
\hline$C(5)$ & $C(4)$ & $C(6)$ & 112(1) & $C(211)$ & $C(216)$ & $C(215)$ & $120(1)$ \\
\hline$C(5)$ & $C(4)$ & $C(7)$ & 112(1) & $P(3)$ & $C(311)$ & $C(312)$ & $120.5(9)$ \\
\hline$C(6)$ & $C(4)$ & $C(7)$ & 112(1) & $\mathrm{P}(3)$ & $C(311)$ & $C(316)$ & $120.3(9)$ \\
\hline$P(1)$ & $C(111)$ & $C(112)$ & $118.9(8)$ & $C(312)$ & $C(311)$ & $C(316)$ & $119(1)$ \\
\hline$P(1)$ & $C(111)$ & $C(116)$ & 121.1(9) & $C(311)$ & $C(312)$ & $C(313)$ & $120(1)$ \\
\hline$C(112)$ & $C(111)$ & $C(116)$ & $120(1)$ & $C(312)$ & $C(313)$ & $C(314)$ & $122(1)$ \\
\hline$C(111)$ & $C(112)$ & $C(113)$ & 121(1) & $C(313)$ & $C(314)$ & $C(315)$ & $120(1)$ \\
\hline$C(112)$ & $C(113)$ & $C(114)$ & 118(1) & $C(314)$ & $C(315)$ & $C(316)$ & 119(1) \\
\hline
\end{tabular}




\begin{tabular}{llllllll}
$\mathrm{C}(113)$ & $\mathrm{C}(114)$ & $\mathrm{C}(115)$ & $121(1)$ & $\mathrm{C}(311)$ & $\mathrm{C}(316)$ & $\mathrm{C}(315)$ & $120(1)$ \\
$\mathrm{C}(114)$ & $\mathrm{C}(115)$ & $\mathrm{C}(116)$ & $121(1)$ & $\mathrm{P}(3)$ & $\mathrm{C}(321)$ & $\mathrm{C}(322)$ & $120.1(8)$ \\
$\mathrm{C}(111)$ & $\mathrm{C}(116)$ & $\mathrm{C}(115)$ & $119(1)$ & $\mathrm{P}(3)$ & $\mathrm{C}(321)$ & $\mathrm{C}(326)$ & $121.4(9)$ \\
$\mathrm{P}(1)$ & $\mathrm{C}(121)$ & $\mathrm{C}(122)$ & $123(1)$ & $\mathrm{C}(322)$ & $\mathrm{C}(321)$ & $\mathrm{C}(326)$ & $118(1)$ \\
$\mathrm{P}(1)$ & $\mathrm{C}(121)$ & $\mathrm{C}(126)$ & $118.6(8)$ & $\mathrm{C}(321)$ & $\mathrm{C}(322)$ & $\mathrm{C}(323)$ & $122(1)$ \\
$\mathrm{C}(122)$ & $\mathrm{C}(121)$ & $\mathrm{C}(126)$ & $118(1)$ & $\mathrm{C}(322)$ & $\mathrm{C}(323)$ & $\mathrm{C}(324)$ & $119(1)$ \\
$\mathrm{C}(121)$ & $\mathrm{C}(122)$ & $\mathrm{C}(123)$ & $118(1)$ & $\mathrm{C}(323)$ & $\mathrm{C}(324)$ & $\mathrm{C}(325)$ & $120(1)$ \\
$\mathrm{C}(122)$ & $\mathrm{C}(123)$ & $\mathrm{C}(124)$ & $123(1)$ & $\mathrm{C}(324)$ & $\mathrm{C}(325)$ & $\mathrm{C}(326)$ & $121(1)$ \\
$\mathrm{C}(123)$ & $\mathrm{C}(124)$ & $\mathrm{C}(125)$ & $120(1)$ & $\mathrm{C}(321)$ & $\mathrm{C}(326)$ & $\mathrm{C}(325)$ & $120(1)$ \\
$\mathrm{C}(124)$ & $\mathrm{C}(125)$ & $\mathrm{C}(126)$ & $119(1)$ & & & & \\
$\mathrm{C}(121)$ & $\mathrm{C}(126)$ & $\mathrm{C}(125)$ & $122(1)$ & & & & \\
$\mathrm{P}(2)$ & $\mathrm{C}(211)$ & $\mathrm{C}(212)$ & $122.9(8)$ & & & & \\
$\mathrm{P}(2)$ & $\mathrm{C}(211)$ & $\mathrm{C}(216)$ & $115.9(8)$ & & & & \\
$\mathrm{C}(212)$ & $\mathrm{C}(211)$ & $\mathrm{C}(216)$ & $121(1)$ & & & & \\
$\mathrm{C}(211)$ & $\mathrm{C}(212)$ & $\mathrm{C}(213)$ & $120(1)$ & & & & \\
$\mathrm{C}(212)$ & $\mathrm{C}(213)$ & $\mathrm{C}(214)$ & $120(1)$ & & & & \\
$\mathrm{C}(213)$ & $\mathrm{C}(214)$ & $\mathrm{C}(215)$ & $120(1)$ & & & & \\
$\mathrm{C}(214)$ & $\mathrm{C}(215)$ & $\mathrm{C}(216)$ & $120(1)$ & & & & \\
\hline
\end{tabular}

a Estimated standard deviations are given in parentheses. 
Table S4-1. Crystallographic and Experimental Data for $\mathbf{6 a} \cdot\left(\mathrm{CH}_{3}\right)_{2} \mathrm{CO} \cdot \mathrm{Et}_{2} \mathrm{O}$

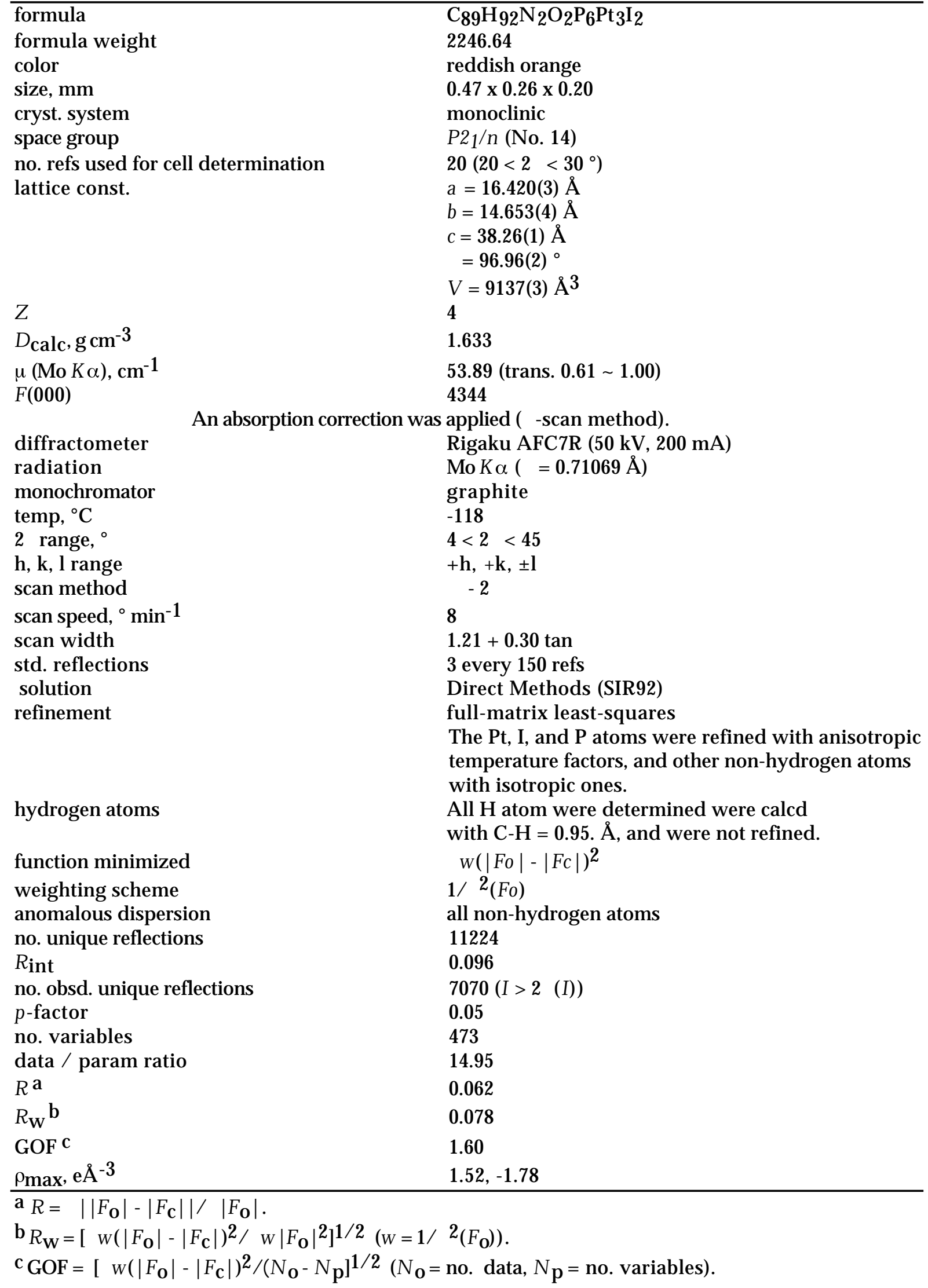


Table S4-2. Final Positional Parameters and $B_{\text {eq }}$ for $6 \mathbf{a} \cdot\left(\mathrm{CH}_{3}\right)_{2} \mathrm{CO} \cdot \mathrm{Et}_{2} \mathrm{O}^{\mathrm{a}}, \mathrm{b}, \mathrm{c}$

\begin{tabular}{|c|c|c|c|c|}
\hline atom & $x$ & $y$ & $z$ & $B_{\mathrm{eq}}$ \\
\hline$\overline{P t}(1)$ & $1.04511(5)$ & $0.22028(6)$ & $0.14455(2)$ & $2.82(2)$ \\
\hline $\operatorname{Pt}(2)$ & $0.90162(5)$ & $0.31245(6)$ & $0.12934(2)$ & $2.72(2)$ \\
\hline $\operatorname{Pt}(3)$ & $0.75755(5)$ & $0.40914(6)$ & $0.11724(2)$ & $2.80(2)$ \\
\hline $\mathrm{I}(1)$ & $0.67260(9)$ & $-0.0389(1)$ & $0.18058(4)$ & $3.76(4)$ \\
\hline $\mathrm{I}(2)$ & $1.1333(1)$ & $0.6890(1)$ & $0.09601(5)$ & $5.35(5)$ \\
\hline $\mathrm{P}(1)$ & $0.9697(3)$ & $0.1190(4)$ & $0.1720(1)$ & $3.0(1)$ \\
\hline$P(2)$ & $0.8283(3)$ & $0.1820(4)$ & $0.1247(1)$ & $3.1(1)$ \\
\hline $\mathrm{P}(3)$ & $0.6863(4)$ & $0.2901(4)$ & $0.1387(1)$ & $3.3(1)$ \\
\hline $\mathrm{P}(4)$ & $1.0978(4)$ & $0.3362(4)$ & $0.1139(1)$ & $3.4(1)$ \\
\hline$P(5)$ & $0.9666(3)$ & $0.4482(4)$ & $0.1330(1)$ & $2.9(1)$ \\
\hline$P(6)$ & $0.8246(3)$ & $0.5216(4)$ & $0.0907(1)$ & $3.0(1)$ \\
\hline $\mathrm{O}(701)$ & $0.695(1)$ & $0.016(1)$ & $0.7144(5)$ & $6.6(4)$ \\
\hline $\mathrm{O}(702)$ & $0.414(1)$ & $0.217(2)$ & $0.9686(7)$ & $10.3(7)$ \\
\hline $\mathrm{N}(1)$ & $1.214(1)$ & $0.115(1)$ & $0.1563(5)$ & $4.9(4)$ \\
\hline $\mathrm{N}(2)$ & $0.593(1)$ & $0.520(1)$ & $0.1086(4)$ & $3.6(4)$ \\
\hline$C(1)$ & $1.149(1)$ & $0.151(1)$ & $0.1527(5)$ & $2.4(4)$ \\
\hline$C(2)$ & $0.656(1)$ & $0.481(1)$ & $0.1108(5)$ & $2.4(4)$ \\
\hline$C(3)$ & $0.884(1)$ & $0.078(1)$ & $0.1409(5)$ & $2.7(4)$ \\
\hline$C(4)$ & $0.750(1)$ & $0.187(1)$ & $0.1544(5)$ & $3.6(5)$ \\
\hline$C(5)$ & $1.081(1)$ & $0.445(1)$ & $0.1332(5)$ & $2.5(4)$ \\
\hline$C(6)$ & $0.935(1)$ & $0.508(1)$ & $0.0919(5)$ & $3.3(4)$ \\
\hline$C(11)$ & $1.291(2)$ & $0.072(2)$ & $0.1604(7)$ & $5.1(6)$ \\
\hline$C(12)$ & $1.290(2)$ & $-0.013(2)$ & $0.1423(8)$ & $6.9(7)$ \\
\hline$C(13)$ & $1.368(2)$ & $-0.060(3)$ & $0.146(1)$ & $10.4(10)$ \\
\hline$C(14)$ & $1.425(2)$ & $-0.013(3)$ & $0.1662(10)$ & $9.2(9)$ \\
\hline$C(15)$ & $1.435(2)$ & $0.065(3)$ & $0.1837(9)$ & $8.5(8)$ \\
\hline$C(16)$ & $1.354(2)$ & $0.117(2)$ & $0.1813(8)$ & $7.7(8)$ \\
\hline$C(17)$ & $1.225(2)$ & $-0.051(3)$ & $0.121(1)$ & $11(1)$ \\
\hline$C(18)$ & $1.342(2)$ & $0.196(2)$ & $0.1960(8)$ & $7.7(8)$ \\
\hline$C(21)$ & $0.522(1)$ & $0.575(2)$ & $0.1075(6)$ & $3.7(5)$ \\
\hline$C(22)$ & $0.500(1)$ & $0.607(2)$ & $0.1404(6)$ & $4.6(5)$ \\
\hline$C(23)$ & $0.427(2)$ & $0.658(2)$ & $0.1386(7)$ & $5.6(6)$ \\
\hline$C(24)$ & $0.385(2)$ & $0.670(2)$ & $0.1068(8)$ & $6.1(6)$ \\
\hline$C(25)$ & $0.404(2)$ & $0.639(2)$ & $0.0749(8)$ & $6.7(7)$ \\
\hline$C(26)$ & $0.479(1)$ & $0.585(2)$ & $0.0738(7)$ & $5.2(6)$ \\
\hline$C(27)$ & $0.553(2)$ & $0.589(2)$ & $0.1725(7)$ & $5.6(6)$ \\
\hline$C(28)$ & $0.502(2)$ & $0.549(2)$ & $0.0414(7)$ & $5.6(6)$ \\
\hline$C(111)$ & $1.024(1)$ & $0.016(1)$ & $0.1876(5)$ & $3.1(4)$ \\
\hline$C(112)$ & $1.076(1)$ & $0.019(2)$ & $0.2202(6)$ & $4.1(5)$ \\
\hline$C(113)$ & $1.125(1)$ & $-0.056(2)$ & $0.2300(6)$ & $4.5(5)$ \\
\hline$C(114)$ & $1.125(1)$ & $-0.129(2)$ & $0.2076(6)$ & $4.6(5)$ \\
\hline$C(115)$ & $1.075(1)$ & $-0.134(2)$ & $0.1782(6)$ & $4.6(5)$ \\
\hline$C(116)$ & $1.024(1)$ & $-0.062(1)$ & $0.1662(5)$ & $3.7(5)$ \\
\hline$C(121)$ & $0.922(1)$ & $0.162(2)$ & $0.2091(6)$ & $3.7(5)$ \\
\hline$C(122)$ & $0.943(1)$ & $0.248(1)$ & $0.2230(5)$ & $3.0(4)$ \\
\hline$C(123)$ & $0.903(1)$ & $0.285(2)$ & $0.2504(6)$ & $3.8(5)$ \\
\hline$C(124)$ & $0.846(1)$ & $0.232(2)$ & $0.2646(5)$ & $3.6(5)$ \\
\hline$C(125)$ & $0.826(1)$ & $0.145(2)$ & $0.2518(6)$ & $3.7(5)$ \\
\hline$C(126)$ & $0.866(1)$ & $0.110(1)$ & $0.2253(5)$ & $3.1(4)$ \\
\hline$C(211)$ & $0.782(1)$ & $0.148(1)$ & $0.0817(5)$ & $3.2(4)$ \\
\hline$C(212)$ & $0.733(1)$ & $0.069(1)$ & $0.0778(5)$ & $3.4(4)$ \\
\hline$C(213)$ & $0.696(1)$ & $0.043(2)$ & $0.0447(6)$ & $4.9(6)$ \\
\hline$C(214)$ & $0.708(1)$ & $0.098(2)$ & $0.0162(7)$ & $5.2(6)$ \\
\hline$C(215)$ & $0.758(1)$ & $0.172(2)$ & $0.0195(6)$ & $4.8(5)$ \\
\hline$C(216)$ & $0.792(1)$ & $0.198(2)$ & $0.0526(6)$ & $3.7(5)$ \\
\hline
\end{tabular}




\begin{tabular}{|c|c|c|c|c|}
\hline$C(311)$ & $0.606(1)$ & $0.244(1)$ & $0.1066(5)$ & $2.4(4)$ \\
\hline$C(312)$ & $0.594(1)$ & $0.275(2)$ & $0.0728(6)$ & $4.1(5)$ \\
\hline$C(313)$ & $0.538(1)$ & $0.242(2)$ & $0.0467(6)$ & $4.3(5)$ \\
\hline$C(314)$ & $0.494(1)$ & $0.165(2)$ & $0.0561(6)$ & $4.0(5)$ \\
\hline$C(315)$ & $0.504(1)$ & $0.131(2)$ & $0.0892(6)$ & $3.8(5)$ \\
\hline$C(316)$ & $0.562(1)$ & $0.169(1)$ & $0.1147(5)$ & $3.2(4)$ \\
\hline$C(321)$ & $0.637(1)$ & $0.324(1)$ & $0.1777(5)$ & $3.2(4)$ \\
\hline$C(322)$ & $0.553(1)$ & $0.330(1)$ & $0.1779(5)$ & $3.5(5)$ \\
\hline$C(323)$ & $0.525(1)$ & $0.356(2)$ & $0.2085(6)$ & $4.5(5)$ \\
\hline$C(324)$ & $0.573(1)$ & $0.377(2)$ & $0.2374(6)$ & $4.2(5)$ \\
\hline$C(325)$ & $0.659(1)$ & $0.377(2)$ & $0.2368(6)$ & $4.5(5)$ \\
\hline$C(326)$ & $0.688(1)$ & $0.353(1)$ & $0.2070(6)$ & $3.5(4)$ \\
\hline$C(411)$ & $1.210(1)$ & $0.338(1)$ & $0.1115(5)$ & $3.4(5)$ \\
\hline$C(412)$ & $1.241(1)$ & $0.265(1)$ & $0.0943(5)$ & $3.5(4)$ \\
\hline$C(413)$ & $1.324(1)$ & $0.264(2)$ & $0.0909(6)$ & $4.9(6)$ \\
\hline$C(414)$ & $1.369(1)$ & $0.330(2)$ & $0.1053(6)$ & $3.8(5)$ \\
\hline$C(415)$ & $1.334(2)$ & $0.402(2)$ & $0.1238(7)$ & $6.1(6)$ \\
\hline$C(416)$ & $1.256(2)$ & $0.403(2)$ & $0.1279(7)$ & $6.1(6)$ \\
\hline$C(421)$ & $1.062(1)$ & $0.338(1)$ & $0.0670(5)$ & $2.7(4)$ \\
\hline$C(422)$ & 1.084(1) & $0.404(1)$ & $0.0447(5)$ & $3.5(4)$ \\
\hline$C(423)$ & $1.054(1)$ & $0.404(2)$ & $0.0094(6)$ & $5.0(6)$ \\
\hline$C(424)$ & $1.004(2)$ & $0.334(2)$ & $-0.0040(7)$ & $5.4(6)$ \\
\hline$C(425)$ & $0.979(1)$ & $0.270(2)$ & $0.0180(7)$ & $5.4(6)$ \\
\hline$C(426)$ & $1.005(1)$ & $0.269(2)$ & $0.0548(6)$ & $3.9(5)$ \\
\hline$C(511)$ & $0.951(1)$ & $0.522(1)$ & $0.1703(5)$ & $3.0(4)$ \\
\hline$C(512)$ & $0.903(1)$ & $0.489(1)$ & $0.1945(5)$ & $3.4(4)$ \\
\hline$C(513)$ & $0.888(1)$ & $0.540(2)$ & $0.2242(6)$ & $3.7(5)$ \\
\hline$C(514)$ & $0.921(1)$ & $0.628(2)$ & $0.2261(6)$ & $4.2(5)$ \\
\hline$C(515)$ & $0.970(1)$ & $0.661(2)$ & $0.2020(6)$ & $4.1(5)$ \\
\hline$C(516)$ & $0.982(1)$ & $0.610(1)$ & $0.1732(5)$ & $3.4(4)$ \\
\hline$C(611)$ & $0.786(1)$ & $0.524(1)$ & $0.0443(5)$ & $2.8(4)$ \\
\hline$C(612)$ & $0.734(1)$ & $0.596(2)$ & $0.0300(6)$ & $4.0(5)$ \\
\hline$C(613)$ & $0.702(1)$ & $0.591(2)$ & $-0.0056(7)$ & $5.2(6)$ \\
\hline$C(614)$ & $0.721(1)$ & $0.523(2)$ & $-0.0274(6)$ & $4.5(5)$ \\
\hline$C(615)$ & $0.772(1)$ & $0.453(2)$ & $-0.0126(6)$ & $4.5(5)$ \\
\hline$C(616)$ & $0.802(1)$ & $0.452(2)$ & $0.0229(6)$ & $3.9(5)$ \\
\hline$C(621)$ & $0.817(1)$ & $0.638(1)$ & $0.1068(6)$ & $3.7(5)$ \\
\hline$C(622)$ & $0.782(1)$ & $0.654(2)$ & $0.1386(6)$ & $3.8(5)$ \\
\hline$C(623)$ & $0.786(1)$ & $0.740(2)$ & $0.1520(6)$ & $4.4(5)$ \\
\hline$C(624)$ & $0.819(2)$ & $0.811(2)$ & $0.1353(7)$ & $5.5(6)$ \\
\hline$C(625)$ & $0.851(1)$ & $0.795(2)$ & $0.1044(6)$ & $4.7(5)$ \\
\hline$C(626)$ & $0.853(1)$ & $0.710(2)$ & $0.0896(6)$ & $4.2(5)$ \\
\hline$C(711)$ & $0.759(2)$ & $-0.115(2)$ & $0.6957(9)$ & $8.8(9)$ \\
\hline$C(712)$ & $0.708(2)$ & $-0.077(2)$ & $0.7183(9)$ & $8.3(8)$ \\
\hline$C(713)$ & $0.647(2)$ & $0.051(3)$ & $0.7373(9)$ & $8.8(9)$ \\
\hline$C(714)$ & $0.649(2)$ & $0.145(3)$ & $0.737(1)$ & $11(1)$ \\
\hline$C(721)$ & $0.507(2)$ & $0.122(2)$ & $0.9471(9)$ & $8.4(8)$ \\
\hline$C(723)$ & $0.396(2)$ & $0.198(2)$ & $0.9081(9)$ & $8.6(8)$ \\
\hline$C(724)$ & $0.436(2)$ & $0.180(2)$ & $0.9433(8)$ & $6.5(7)$ \\
\hline $\mathrm{H}(1)$ & 0.9025 & 0.0473 & 0.1222 & 3.4 \\
\hline $\mathrm{H}(2)$ & 0.8485 & 0.0410 & 0.1527 & 3.4 \\
\hline $\mathrm{H}(3)$ & 0.7160 & 0.1347 & 0.1525 & 4.5 \\
\hline $\mathrm{H}(4)$ & 0.7725 & 0.1962 & 0.1780 & 4.5 \\
\hline $\mathrm{H}(5)$ & 1.1082 & 0.4503 & 0.1558 & 3.3 \\
\hline $\mathrm{H}(6)$ & 1.0981 & 0.4934 & 0.1185 & 3.3 \\
\hline $\mathrm{H}(7)$ & 0.9621 & 0.5659 & 0.0918 & 3.6 \\
\hline $\mathrm{H}(8)$ & 0.9486 & 0.4731 & 0.0724 & 3.6 \\
\hline $\mathrm{H}(9)$ & 1.3801 & -0.1062 & 0.1324 & 12.1 \\
\hline
\end{tabular}




\begin{tabular}{|c|c|c|c|c|}
\hline $\mathrm{H}(10)$ & 1.4753 & -0.0467 & 0.1732 & 25.8 \\
\hline $\mathrm{H}(11)$ & 1.4823 & 0.0890 & 0.1997 & 19.8 \\
\hline $\mathrm{H}(12)$ & 0.4134 & 0.6774 & 0.1616 & 7.3 \\
\hline $\mathrm{H}(13)$ & 0.3342 & 0.7065 & 0.1087 & 8.1 \\
\hline $\mathrm{H}(14)$ & 0.3722 & 0.6553 & 0.0551 & 9.5 \\
\hline $\mathrm{H}(15)$ & 1.1832 & -0.0617 & 0.1359 & 15.6 \\
\hline $\mathrm{H}(16)$ & 1.2079 & -0.0108 & 0.1032 & 15.6 \\
\hline $\mathrm{H}(17)$ & 1.2421 & -0.1076 & 0.1125 & 15.6 \\
\hline $\mathrm{H}(18)$ & 1.3926 & 0.2201 & 0.2082 & 22.2 \\
\hline $\mathrm{H}(19)$ & 1.3263 & 0.2419 & 0.1769 & 22.2 \\
\hline $\mathrm{H}(20)$ & 1.3010 & 0.2001 & 0.2112 & 22.2 \\
\hline $\mathrm{H}(21)$ & 0.5615 & 0.5256 & 0.1755 & 7.2 \\
\hline $\mathrm{H}(22)$ & 0.6049 & 0.6183 & 0.1713 & 7.2 \\
\hline $\mathrm{H}(23)$ & 0.5289 & 0.6136 & 0.1916 & 7.2 \\
\hline $\mathrm{H}(24)$ & 0.5110 & 0.4833 & 0.0435 & 6.6 \\
\hline $\mathrm{H}(25)$ & 0.4652 & 0.5621 & 0.0224 & 6.6 \\
\hline $\mathrm{H}(26)$ & 0.5563 & 0.5741 & 0.0379 & 6.6 \\
\hline $\mathrm{H}(27)$ & 1.0755 & 0.0724 & 0.2348 & 5.1 \\
\hline $\mathrm{H}(28)$ & 1.1606 & -0.0529 & 0.2518 & 5.8 \\
\hline $\mathrm{H}(29)$ & 1.1630 & -0.1793 & 0.2157 & 6.2 \\
\hline $\mathrm{H}(30)$ & 1.0751 & -0.1881 & 0.1644 & 5.4 \\
\hline $\mathrm{H}(31)$ & 0.9912 & -0.0636 & 0.1447 & 4.5 \\
\hline $\mathrm{H}(32)$ & 0.9830 & 0.2810 & 0.2131 & 3.5 \\
\hline $\mathrm{H}(33)$ & 0.9162 & 0.3440 & 0.2582 & 4.6 \\
\hline $\mathrm{H}(34)$ & 0.8207 & 0.2553 & 0.2832 & 4.6 \\
\hline $\mathrm{H}(35)$ & 0.7853 & 0.1105 & 0.2611 & 4.7 \\
\hline $\mathrm{H}(36)$ & 0.8540 & 0.0501 & 0.2175 & 3.6 \\
\hline $\mathrm{H}(37)$ & 0.7231 & 0.0334 & 0.0982 & 3.9 \\
\hline $\mathrm{H}(38)$ & 0.6611 & -0.0100 & 0.0431 & 6.2 \\
\hline $\mathrm{H}(39)$ & 0.6795 & 0.0748 & -0.0050 & 7.3 \\
\hline $\mathrm{H}(40)$ & 0.7634 & 0.2017 & -0.0017 & 6.1 \\
\hline $\mathrm{H}(41)$ & 0.8211 & 0.2516 & 0.0549 & 4.7 \\
\hline $\mathrm{H}(42)$ & 0.6260 & 0.3277 & 0.0675 & 4.5 \\
\hline $\mathrm{H}(43)$ & 0.5318 & 0.2682 & 0.0248 & 4.7 \\
\hline $\mathrm{H}(44)$ & 0.4584 & 0.1391 & 0.0387 & 4.7 \\
\hline $\mathrm{H}(45)$ & 0.4726 & 0.0821 & 0.0956 & 4.4 \\
\hline $\mathrm{H}(46)$ & 0.5729 & 0.1445 & 0.1372 & 3.9 \\
\hline $\mathrm{H}(47)$ & 0.5185 & 0.3165 & 0.1563 & 4.7 \\
\hline $\mathrm{H}(48)$ & 0.4670 & 0.3571 & 0.2083 & 5.7 \\
\hline $\mathrm{H}(49)$ & 0.5523 & 0.3920 & 0.2587 & 5.1 \\
\hline $\mathrm{H}(50)$ & 0.6979 & 0.3944 & 0.2567 & 5.4 \\
\hline $\mathrm{H}(51)$ & 0.7458 & 0.3533 & 0.2054 & 4.5 \\
\hline $\mathrm{H}(52)$ & 1.2036 & 0.2191 & 0.0840 & 4.3 \\
\hline $\mathrm{H}(53)$ & 1.3491 & 0.2190 & 0.0783 & 6.0 \\
\hline $\mathrm{H}(54)$ & 1.4278 & 0.3288 & 0.1059 & 4.8 \\
\hline $\mathrm{H}(55)$ & 1.3676 & 0.4525 & 0.1314 & 9.9 \\
\hline $\mathrm{H}(56)$ & 1.2256 & 0.4487 & 0.1409 & 9.8 \\
\hline $\mathrm{H}(57)$ & 1.1225 & 0.4506 & 0.0543 & 4.4 \\
\hline $\mathrm{H}(58)$ & 1.0700 & 0.4556 & -0.0040 & 6.8 \\
\hline $\mathrm{H}(59)$ & 0.9862 & 0.3349 & -0.0288 & 7.1 \\
\hline $\mathrm{H}(60)$ & 0.9418 & 0.2266 & 0.0088 & 7.2 \\
\hline $\mathrm{H}(61)$ & 0.9895 & 0.2249 & 0.0700 & 4.5 \\
\hline $\mathrm{H}(62)$ & 0.8801 & 0.4294 & 0.1901 & 3.8 \\
\hline $\mathrm{H}(63)$ & 0.8583 & 0.5142 & 0.2412 & 4.5 \\
\hline $\mathrm{H}(64)$ & 0.9078 & 0.6671 & 0.2439 & 5.0 \\
\hline $\mathrm{H}(65)$ & 0.9950 & 0.7200 & 0.2049 & 4.9 \\
\hline $\mathrm{H}(66)$ & 1.0126 & 0.6343 & 0.1557 & 3.9 \\
\hline $\mathrm{H}(67)$ & 0.7567 & 0.6051 & 0.1493 & 4.3 \\
\hline
\end{tabular}




\begin{tabular}{|c|c|c|c|c|}
\hline $\mathrm{H}(68)$ & 0.7600 & 0.7505 & 0.1722 & 5.1 \\
\hline $\mathrm{H}(69)$ & 0.8222 & 0.8693 & 0.1444 & 7.2 \\
\hline $\mathrm{H}(70)$ & 0.8776 & 0.8397 & 0.0931 & 5.8 \\
\hline $\mathrm{H}(71)$ & 0.8795 & 0.6982 & 0.0700 & 5.4 \\
\hline $\mathrm{H}(72)$ & 0.7173 & 0.6450 & 0.0446 & 4.7 \\
\hline $\mathrm{H}(73)$ & 0.6657 & 0.6371 & -0.0142 & 6.3 \\
\hline $\mathrm{H}(74)$ & 0.7030 & 0.5222 & -0.0512 & 5.6 \\
\hline $\mathrm{H}(75)$ & 0.7861 & 0.4011 & -0.0261 & 5.3 \\
\hline $\mathrm{H}(76)$ & 0.8353 & 0.4031 & 0.0325 & 4.3 \\
\hline $\mathrm{H}(77)$ & 0.7682 & -0.1804 & 0.6990 & 8.9 \\
\hline $\mathrm{H}(78)$ & 0.7378 & -0.1066 & 0.6714 & 8.9 \\
\hline $\mathrm{H}(79)$ & 0.8134 & -0.0875 & 0.6988 & 8.9 \\
\hline $\mathrm{H}(80)$ & 0.7065 & 0.1664 & 0.7444 & 11.0 \\
\hline $\mathrm{H}(81)$ & 0.6325 & 0.1692 & 0.7151 & 11.0 \\
\hline $\mathrm{H}(82)$ & 0.6179 & 0.1743 & 0.7543 & 11.0 \\
\hline $\mathrm{H}(83)$ & 0.4966 & 0.0690 & 0.9603 & 8.8 \\
\hline $\mathrm{H}(84)$ & 0.5158 & 0.0961 & 0.9230 & 8.8 \\
\hline $\mathrm{H}(85)$ & 0.5543 & 0.1507 & 0.9556 & 8.8 \\
\hline $\mathrm{H}(86)$ & 0.4003 & 0.2618 & 0.9023 & 9.3 \\
\hline $\mathrm{H}(87)$ & 0.4209 & 0.1644 & 0.8902 & 9.3 \\
\hline $\mathrm{H}(88)$ & 0.3399 & 0.1821 & 0.9062 & 9.3 \\
\hline $\mathrm{H}(89)$ & 0.7335 & -0.0923 & 0.7418 & 8.7 \\
\hline $\mathrm{H}(90)$ & 0.6579 & -0.1111 & 0.7142 & 8.7 \\
\hline $\mathrm{H}(91)$ & 0.5900 & 0.0347 & 0.7333 & 9.4 \\
\hline $\mathrm{H}(92)$ & 0.6644 & 0.0319 & 0.7624 & 9.4 \\
\hline
\end{tabular}

$\mathrm{a}$ Estimated standard deviations are given in parentheses. ${ }^{\mathrm{b}}$ The $\mathrm{Pt}, \mathrm{I}$, and $\mathrm{P}$ atoms were assigned anisotropic thermal parameters given as the isotropic equivalent displacement parameter defined as $B_{\mathrm{eq}}=\left(8 \pi^{2} / 3\right)\left(U_{11}\left(a a^{*}\right)^{2}+U_{22}\left(b b^{*}\right)^{2}+U_{33}\left(c c^{*}\right)^{2}+2 U_{12} a a^{*} b b^{*} \cos \gamma+2 U_{13} a a^{*} c c^{*} \cos \beta+\right.$ $\left.2 U_{23} b b^{*} c c^{*} \cos \alpha\right)$. Other non-hydrogen atoms were refined isotropically. ${ }^{c}$ The position of all hydrogen atoms were calculated with $\mathrm{C}-\mathrm{H}=0.95 \AA$, and were not refined with the appropriate $B$ iso.

Table S4-3. Final Anisotropic Thermal Parameters for 6a $\cdot\left(\mathrm{CH}_{3}\right)_{2} \mathrm{CO} \cdot \mathrm{Et}_{2} \mathrm{O}{ }^{\mathrm{a}}, \mathrm{b}$

\begin{tabular}{llllccc}
\hline atom & $U_{11}$ & $U_{22}$ & $U_{33}$ & $U_{12}$ & $U_{13}$ & $U_{23}$ \\
\hline $\mathrm{Pt}(1)$ & $0.0319(5)$ & $0.0336(5)$ & $0.0423(5)$ & $0.0001(4)$ & $0.0067(4)$ & $0.0007(4)$ \\
$\mathrm{Pt}(2)$ & $0.0336(5)$ & $0.0320(5)$ & $0.0384(5)$ & $0.0006(4)$ & $0.0076(4)$ & $0.0001(4)$ \\
$\mathrm{Pt}(3)$ & $0.0337(5)$ & $0.0338(5)$ & $0.0396(5)$ & $0.0015(4)$ & $0.0078(4)$ & $0.0027(4)$ \\
$\mathrm{I}(1)$ & $0.0477(10)$ & $0.0409(9)$ & $0.0553(10)$ & $0.0009(8)$ & $0.0110(7)$ & $0.0039(7)$ \\
$\mathrm{I}(2)$ & $0.070(1)$ & $0.068(1)$ & $0.066(1)$ & $-0.007(1)$ & $0.0098(9)$ & $-0.0068(10)$ \\
$\mathrm{P}(1)$ & $0.032(3)$ & $0.039(3)$ & $0.045(3)$ & $0.004(3)$ & $0.009(3)$ & $-0.003(3)$ \\
$\mathrm{P}(2)$ & $0.040(4)$ & $0.037(3)$ & $0.038(3)$ & $-0.002(3)$ & $0.000(3)$ & $-0.002(3)$ \\
$\mathrm{P}(3)$ & $0.043(4)$ & $0.045(4)$ & $0.040(3)$ & $-0.014(3)$ & $0.011(3)$ & $-0.002(3)$ \\
$\mathrm{P}(4)$ & $0.051(4)$ & $0.040(4)$ & $0.039(3)$ & $-0.001(3)$ & $0.008(3)$ & $-0.001(3)$ \\
$\mathrm{P}(5)$ & $0.042(4)$ & $0.037(3)$ & $0.033(3)$ & $-0.001(3)$ & $0.004(3)$ & $-0.004(3)$ \\
$\mathrm{P}(6)$ & $0.034(4)$ & $0.038(4)$ & $0.043(3)$ & $0.003(3)$ & $0.007(3)$ & $-0.001(3)$ \\
\hline
\end{tabular}

a Estmated standard deviations are given in parentheses. $\mathrm{b}$ Parameters have the form exp($\left.2 \pi^{2}\left(U_{11} h^{2} a^{* 2}+\cdots+2 U_{12} h k a^{*} b^{*} \cos \gamma+\cdots\right)\right)$

Table S4-4. Bond Lengths for Non-hydrogen Atoms of $6 \mathbf{a} \cdot\left(\mathrm{CH}_{3}\right)_{2} \mathrm{CO} \cdot \mathrm{Et}_{2} \mathrm{O}^{\mathrm{a}}$

\begin{tabular}{|c|c|c|c|c|c|}
\hline atom & atom & distance & atom & atom & distance \\
\hline $\operatorname{Pt}(1)$ & $\operatorname{Pt}(2)$ & $2.717(1)$ & $\mathrm{P}(6)$ & $C(611)$ & $1.81(2)$ \\
\hline $\operatorname{Pt}(1)$ & $P(1)$ & $2.271(6)$ & $P(6)$ & $C(621)$ & $1.83(2)$ \\
\hline
\end{tabular}




\begin{tabular}{|c|c|c|c|c|c|}
\hline $\operatorname{Pt}(1)$ & $\mathrm{P}(4)$ & $2.291(6)$ & $\mathrm{O}(701)$ & $C(712)$ & $1.38(3)$ \\
\hline $\operatorname{Pt}(1)$ & $C(1)$ & $1.98(2)$ & $\mathrm{O}(701)$ & $C(713)$ & $1.36(4)$ \\
\hline $\operatorname{Pt}(2)$ & $\operatorname{Pt}(3)$ & $2.748(1)$ & $\mathrm{O}(702)$ & $C(724)$ & $1.20(3)$ \\
\hline $\operatorname{Pt}(2)$ & $\mathrm{P}(2)$ & $2.254(6)$ & $\mathrm{N}(1)$ & $C(1)$ & $1.18(2)$ \\
\hline $\operatorname{Pt}(2)$ & $P(5)$ & $2.254(6)$ & $\mathrm{N}(1)$ & $C(11)$ & $1.40(3)$ \\
\hline $\operatorname{Pt}(3)$ & $P(3)$ & $2.306(6)$ & $N(2)$ & $C(2)$ & $1.17(2)$ \\
\hline $\operatorname{Pt}(3)$ & $\mathrm{P}(6)$ & $2.288(6)$ & $\mathrm{N}(2)$ & $C(21)$ & $1.42(3)$ \\
\hline $\operatorname{Pt}(3)$ & $C(2)$ & $1.97(2)$ & $C(11)$ & $C(12)$ & $1.42(4)$ \\
\hline $\mathrm{P}(1)$ & $C(3)$ & $1.83(2)$ & $C(11)$ & $C(16)$ & $1.40(4)$ \\
\hline $\mathrm{P}(1)$ & $C(111)$ & $1.81(2)$ & $C(12)$ & $C(13)$ & $1.44(4)$ \\
\hline $\mathrm{P}(1)$ & $C(121)$ & $1.81(2)$ & $C(12)$ & $C(17)$ & $1.39(4)$ \\
\hline $\mathrm{P}(2)$ & $C(3)$ & $1.85(2)$ & $C(13)$ & $C(14)$ & $1.33(4)$ \\
\hline $\mathrm{P}(2)$ & $C(4)$ & $1.82(2)$ & $C(14)$ & $C(15)$ & $1.32(4)$ \\
\hline $\mathrm{P}(2)$ & $C(211)$ & $1.80(2)$ & $C(15)$ & $C(16)$ & $1.53(4)$ \\
\hline $\mathrm{P}(3)$ & $C(4)$ & $1.89(2)$ & $C(16)$ & $C(18)$ & $1.31(4)$ \\
\hline $\mathrm{P}(3)$ & $C(311)$ & $1.82(2)$ & $C(21)$ & $C(22)$ & $1.42(3)$ \\
\hline $\mathrm{P}(3)$ & $C(321)$ & $1.84(2)$ & $C(21)$ & $C(26)$ & $1.40(3)$ \\
\hline $\mathrm{P}(4)$ & $C(5)$ & $1.79(2)$ & $C(22)$ & $C(23)$ & $1.41(3)$ \\
\hline $\mathrm{P}(4)$ & $C(411)$ & $1.86(2)$ & $C(22)$ & $C(27)$ & $1.44(3)$ \\
\hline $\mathrm{P}(4)$ & $C(421)$ & $1.82(2)$ & $C(23)$ & $C(24)$ & $1.34(3)$ \\
\hline $\mathrm{P}(5)$ & $C(5)$ & $1.88(2)$ & $C(24)$ & $C(25)$ & $1.38(4)$ \\
\hline $\mathrm{P}(5)$ & $C(6)$ & $1.82(2)$ & $C(25)$ & $C(26)$ & $1.47(3)$ \\
\hline $\mathrm{P}(5)$ & $C(511)$ & $1.83(2)$ & $C(26)$ & $C(28)$ & $1.44(3)$ \\
\hline $\mathrm{P}(6)$ & $C(6)$ & $1.82(2)$ & $C(111)$ & $C(112)$ & $1.42(3)$ \\
\hline$C(111)$ & $C(116)$ & $1.41(3)$ & $C(323)$ & $C(324)$ & $1.32(3)$ \\
\hline$C(112)$ & $C(113)$ & 1.39(3) & $C(324)$ & $C(325)$ & $1.41(3)$ \\
\hline$C(113)$ & $C(114)$ & $1.38(3)$ & $C(325)$ & $C(326)$ & $1.34(3)$ \\
\hline$C(114)$ & $C(115)$ & $1.31(3)$ & $C(411)$ & $C(412)$ & $1.39(3)$ \\
\hline$C(115)$ & $C(116)$ & 1.39(3) & $C(411)$ & $C(416)$ & $1.31(3)$ \\
\hline$C(121)$ & $C(122)$ & $1.40(3)$ & $C(412)$ & $C(413)$ & $1.38(3)$ \\
\hline$C(121)$ & $C(126)$ & $1.40(3)$ & $C(413)$ & $C(414)$ & $1.31(3)$ \\
\hline$C(122)$ & $C(123)$ & $1.41(3)$ & $C(414)$ & $C(415)$ & $1.43(3)$ \\
\hline$C(123)$ & $C(124)$ & $1.38(3)$ & $C(415)$ & $C(416)$ & $1.30(3)$ \\
\hline$C(124)$ & $C(125)$ & $1.39(3)$ & $C(421)$ & $C(422)$ & $1.37(3)$ \\
\hline$C(125)$ & $C(126)$ & $1.37(3)$ & $C(421)$ & $C(426)$ & $1.41(3)$ \\
\hline$C(211)$ & $C(212)$ & 1.41(3) & $C(422)$ & $C(423)$ & $1.38(3)$ \\
\hline$C(211)$ & $C(216)$ & $1.36(3)$ & $C(423)$ & $C(424)$ & $1.37(3)$ \\
\hline$C(212)$ & $C(213)$ & $1.39(3)$ & $C(424)$ & $C(425)$ & $1.36(3)$ \\
\hline$C(213)$ & $C(214)$ & $1.39(3)$ & $C(425)$ & $C(426)$ & $1.42(3)$ \\
\hline$C(214)$ & $C(215)$ & $1.36(3)$ & $C(511)$ & $C(512)$ & $1.37(3)$ \\
\hline$C(215)$ & $C(216)$ & $1.37(3)$ & $C(511)$ & $C(516)$ & $1.39(3)$ \\
\hline$C(311)$ & $C(312)$ & $1.36(3)$ & $C(512)$ & $C(513)$ & $1.40(3)$ \\
\hline$C(311)$ & $C(316)$ & $1.38(3)$ & $C(513)$ & $C(514)$ & $1.40(3)$ \\
\hline$C(312)$ & $C(313)$ & $1.37(3)$ & $C(514)$ & $C(515)$ & $1.38(3)$ \\
\hline$C(313)$ & $C(314)$ & $1.40(3)$ & $C(515)$ & $C(516)$ & $1.38(3)$ \\
\hline$C(314)$ & $C(315)$ & $1.35(3)$ & $C(611)$ & $C(612)$ & $1.43(3)$ \\
\hline$C(315)$ & $C(316)$ & $1.39(3)$ & $C(611)$ & $C(616)$ & $1.39(3)$ \\
\hline$C(321)$ & $C(322)$ & $1.39(3)$ & $C(612)$ & $C(613)$ & $1.40(3)$ \\
\hline$C(321)$ & $C(326)$ & $1.38(3)$ & $C(613)$ & $C(614)$ & $1.36(3)$ \\
\hline$C(322)$ & $C(323)$ & $1.37(3)$ & $C(614)$ & $C(615)$ & $1.39(3)$ \\
\hline$C(615)$ & $C(616)$ & $1.39(3)$ & & & \\
\hline$C(621)$ & $C(622)$ & $1.43(3)$ & & & \\
\hline$C(621)$ & $C(626)$ & $1.41(3)$ & & & \\
\hline$C(622)$ & $C(623)$ & $1.36(3)$ & & & \\
\hline$C(623)$ & $C(624)$ & $1.37(3)$ & & & \\
\hline$C(624)$ & $C(625)$ & $1.37(3)$ & & & \\
\hline
\end{tabular}




\begin{tabular}{lll}
$\mathrm{C}(625)$ & $\mathrm{C}(626)$ & $1.36(3)$ \\
$\mathrm{C}(711)$ & $\mathrm{C}(712)$ & $1.40(4)$ \\
$\mathrm{C}(713)$ & $\mathrm{C}(714)$ & $1.37(5)$ \\
$\mathrm{C}(721)$ & $\mathrm{C}(724)$ & $1.43(4)$ \\
$\mathrm{C}(723)$ & $\mathrm{C}(724)$ & $1.45(4)$ \\
\hline
\end{tabular}

a Estimated standard deviations are given in parentheses.

Table S4-5. Bond Angles for Non-hydrogen Atoms of $6 \mathbf{a} \cdot\left(\mathrm{CH}_{3}\right)_{2} \mathrm{CO} \cdot \mathrm{Et}_{2} \mathrm{O}^{\mathrm{a}}$

\begin{tabular}{|c|c|c|c|c|c|c|c|}
\hline atom & atom & atom & angle & atom & atom & atom & angle \\
\hline $\operatorname{Pt}(2)$ & $\operatorname{Pt}(1)$ & $\mathrm{P}(1)$ & $85.2(1)$ & $\operatorname{Pt}(2)$ & $\mathrm{P}(2)$ & $C(211)$ & $118.0(7)$ \\
\hline $\operatorname{Pt}(2)$ & $\operatorname{Pt}(1)$ & $\mathrm{P}(4)$ & $83.9(2)$ & $C(3)$ & $\mathrm{P}(2)$ & $C(4)$ & $100.5(9)$ \\
\hline $\operatorname{Pt}(2)$ & $\operatorname{Pt}(1)$ & $C(1)$ & $176.6(5)$ & $C(3)$ & $\mathrm{P}(2)$ & $C(211)$ & $102.8(9)$ \\
\hline$P(1)$ & $\operatorname{Pt}(1)$ & $\mathrm{P}(4)$ & $169.1(2)$ & $C(4)$ & $\mathrm{P}(2)$ & $C(211)$ & 109.1(9) \\
\hline $\mathrm{P}(1)$ & $\operatorname{Pt}(1)$ & $C(1)$ & $95.7(6)$ & $\operatorname{Pt}(3)$ & $\mathrm{P}(3)$ & $C(4)$ & $115.7(7)$ \\
\hline $\mathrm{P}(4)$ & $\operatorname{Pt}(1)$ & $C(1)$ & $95.1(6)$ & $\operatorname{Pt}(3)$ & $P(3)$ & $C(311)$ & $113.2(7)$ \\
\hline $\operatorname{Pt}(1)$ & $\operatorname{Pt}(2)$ & $\operatorname{Pt}(3)$ & $177.19(4)$ & $\operatorname{Pt}(3)$ & $P(3)$ & $C(321)$ & $112.1(7)$ \\
\hline $\operatorname{Pt}(1)$ & $\operatorname{Pt}(2)$ & $P(2)$ & $92.1(1)$ & $C(4)$ & $P(3)$ & $C(311)$ & $104.3(9)$ \\
\hline $\operatorname{Pt}(1)$ & $\operatorname{Pt}(2)$ & $P(5)$ & $91.9(1)$ & $C(4)$ & $P(3)$ & $C(321)$ & $103.5(9)$ \\
\hline $\operatorname{Pt}(3)$ & $\operatorname{Pt}(2)$ & $P(2)$ & $89.0(1)$ & $C(311)$ & $P(3)$ & $C(321)$ & 107.2(9) \\
\hline $\operatorname{Pt}(3)$ & $\operatorname{Pt}(2)$ & $P(5)$ & $87.0(1)$ & $\operatorname{Pt}(1)$ & $\mathrm{P}(4)$ & $C(5)$ & $111.2(7)$ \\
\hline $\mathrm{P}(2)$ & $\operatorname{Pt}(2)$ & $\mathrm{P}(5)$ & $176.0(2)$ & $\operatorname{Pt}(1)$ & $\mathrm{P}(4)$ & $C(411)$ & $118.5(7)$ \\
\hline $\operatorname{Pt}(2)$ & $\operatorname{Pt}(3)$ & $P(3)$ & $90.8(2)$ & $\operatorname{Pt}(1)$ & $\mathrm{P}(4)$ & $C(421)$ & $114.5(7)$ \\
\hline $\operatorname{Pt}(2)$ & $\operatorname{Pt}(3)$ & $P(6)$ & $89.8(1)$ & $C(5)$ & $\mathrm{P}(4)$ & $C(411)$ & 102.1(9) \\
\hline $\operatorname{Pt}(2)$ & $\operatorname{Pt}(3)$ & $C(2)$ & $177.3(5)$ & $C(5)$ & $\mathrm{P}(4)$ & $C(421)$ & $110.3(9)$ \\
\hline $\mathrm{P}(3)$ & $\operatorname{Pt}(3)$ & $P(6)$ & $174.5(2)$ & $C(411)$ & $\mathrm{P}(4)$ & $C(421)$ & $99.0(9)$ \\
\hline$P(3)$ & $\operatorname{Pt}(3)$ & $C(2)$ & $89.2(6)$ & $\operatorname{Pt}(2)$ & $P(5)$ & $C(5)$ & $116.3(6)$ \\
\hline$P(6)$ & $\operatorname{Pt}(3)$ & $C(2)$ & $90.5(6)$ & $\operatorname{Pt}(2)$ & $P(5)$ & $C(6)$ & $106.9(7)$ \\
\hline $\operatorname{Pt}(1)$ & $P(1)$ & $C(3)$ & $109.4(7)$ & $\operatorname{Pt}(2)$ & $P(5)$ & $C(511)$ & $117.1(7)$ \\
\hline $\operatorname{Pt}(1)$ & $P(1)$ & $C(111)$ & $114.8(7)$ & $C(5)$ & $P(5)$ & $C(6)$ & 101.3(9) \\
\hline $\operatorname{Pt}(1)$ & $\mathrm{P}(1)$ & $C(121)$ & $116.4(8)$ & $C(5)$ & $P(5)$ & $C(511)$ & $104.3(9)$ \\
\hline$C(3)$ & $P(1)$ & $C(111)$ & $104.6(9)$ & $C(6)$ & $P(5)$ & $C(511)$ & 109.7(9) \\
\hline$C(3)$ & $\mathrm{P}(1)$ & $C(121)$ & $104.7(9)$ & $\operatorname{Pt}(3)$ & $P(6)$ & $C(6)$ & $116.3(7)$ \\
\hline$C(111)$ & $P(1)$ & $C(121)$ & $106(1)$ & $\operatorname{Pt}(3)$ & $P(6)$ & $C(611)$ & $108.6(7)$ \\
\hline $\operatorname{Pt}(2)$ & $\mathrm{P}(2)$ & $C(3)$ & $115.6(6)$ & $\operatorname{Pt}(3)$ & $P(6)$ & $C(621)$ & $117.9(7)$ \\
\hline $\operatorname{Pt}(2)$ & $\mathrm{P}(2)$ & $C(4)$ & 109.1(7) & $C(6)$ & $P(6)$ & $C(611)$ & $105.0(9)$ \\
\hline$C(6)$ & $P(6)$ & $C(621)$ & $102(1)$ & $C(21)$ & $C(22)$ & $C(23)$ & $116(2)$ \\
\hline$C(611)$ & $P(6)$ & $C(621)$ & $106(1)$ & $C(21)$ & $C(22)$ & $C(27)$ & $120(2)$ \\
\hline$C(712)$ & $\mathrm{O}(701)$ & $C(713)$ & $114(3)$ & $C(23)$ & $C(22)$ & $C(27)$ & $124(2)$ \\
\hline$C(1)$ & $\mathrm{N}(1)$ & $C(11)$ & $179(2)$ & $C(22)$ & $C(23)$ & $C(24)$ & 118(3) \\
\hline$C(2)$ & $\mathrm{N}(2)$ & $C(21)$ & $175(2)$ & $C(23)$ & $C(24)$ & $C(25)$ & $128(3)$ \\
\hline $\operatorname{Pt}(1)$ & $C(1)$ & $\mathrm{N}(1)$ & $175(2)$ & $C(24)$ & $C(25)$ & $C(26)$ & 119(3) \\
\hline $\operatorname{Pt}(3)$ & $C(2)$ & $\mathrm{N}(2)$ & $176(2)$ & $C(21)$ & $C(26)$ & $C(25)$ & $111(2)$ \\
\hline $\mathrm{P}(1)$ & $C(3)$ & $P(2)$ & $105(1)$ & $C(21)$ & $C(26)$ & $C(28)$ & $127(2)$ \\
\hline $\mathrm{P}(2)$ & $C(4)$ & $\mathrm{P}(3)$ & 104(1) & $C(25)$ & $C(26)$ & $C(28)$ & $122(2)$ \\
\hline $\mathrm{P}(4)$ & $C(5)$ & $P(5)$ & 103.1(9) & $\mathrm{P}(1)$ & $C(111)$ & $C(112)$ & 119(2) \\
\hline$P(5)$ & $C(6)$ & $P(6)$ & $105(1)$ & $\mathrm{P}(1)$ & $C(111)$ & $C(116)$ & $121(2)$ \\
\hline $\mathrm{N}(1)$ & $C(11)$ & $C(12)$ & $113(2)$ & $C(112)$ & $C(111)$ & $C(116)$ & $119(2)$ \\
\hline $\mathrm{N}(1)$ & $C(11)$ & $C(16)$ & 116(3) & $C(111)$ & $C(112)$ & $C(113)$ & $119(2)$ \\
\hline$C(12)$ & $C(11)$ & $C(16)$ & 131(3) & $C(112)$ & $C(113)$ & $C(114)$ & $119(2)$ \\
\hline$C(11)$ & $C(12)$ & $C(13)$ & $115(3)$ & $C(113)$ & $C(114)$ & $C(115)$ & $122(2)$ \\
\hline$C(11)$ & $C(12)$ & $C(17)$ & $127(3)$ & $C(114)$ & $C(115)$ & $C(116)$ & $122(2)$ \\
\hline$C(13)$ & $C(12)$ & $C(17)$ & $119(3)$ & $C(111)$ & $C(116)$ & $C(115)$ & $118(2)$ \\
\hline$C(12)$ & $C(13)$ & $C(14)$ & $111(4)$ & $\mathrm{P}(1)$ & $C(121)$ & $C(122)$ & $120(2)$ \\
\hline$C(13)$ & $C(14)$ & $C(15)$ & $142(4)$ & $P(1)$ & $C(121)$ & $C(126)$ & $122(2)$ \\
\hline$C(14)$ & $C(15)$ & $C(16)$ & $109(3)$ & $C(122)$ & $C(121)$ & $C(126)$ & $118(2)$ \\
\hline
\end{tabular}




\begin{tabular}{|c|c|c|c|c|c|c|c|}
\hline$C(11)$ & $C(16)$ & $C(15)$ & $113(3)$ & $C(121)$ & $C(122)$ & $C(123)$ & $121(2)$ \\
\hline$C(11)$ & $C(16)$ & C(18) & $122(3)$ & $C(122)$ & $C(123)$ & $C(124)$ & $118(2)$ \\
\hline$C(15)$ & $C(16)$ & $C(18)$ & $126(3)$ & $C(123)$ & $C(124)$ & $C(125)$ & $122(2)$ \\
\hline $\mathrm{N}(2)$ & $C(21)$ & $C(22)$ & $117(2)$ & $C(124)$ & $C(125)$ & $C(126)$ & $119(2)$ \\
\hline $\mathrm{N}(2)$ & $C(21)$ & $C(26)$ & $114(2)$ & $C(121)$ & $C(126)$ & $C(125)$ & $122(2)$ \\
\hline$C(22)$ & $C(21)$ & $C(26)$ & $129(2)$ & $\mathrm{P}(2)$ & $C(211)$ & $C(212)$ & $120(2)$ \\
\hline $\mathrm{P}(2)$ & $C(211)$ & $C(216)$ & $122(2)$ & $C(411)$ & $C(412)$ & $C(413)$ & $117(2)$ \\
\hline$C(212)$ & $C(211)$ & $C(216)$ & $119(2)$ & $C(412)$ & $C(413)$ & $C(414)$ & $119(2)$ \\
\hline$C(211)$ & $C(212)$ & $C(213)$ & $120(2)$ & $C(413)$ & $C(414)$ & $C(415)$ & $120(2)$ \\
\hline$C(212)$ & $C(213)$ & $C(214)$ & $118(2)$ & $C(414)$ & $C(415)$ & $C(416)$ & $122(3)$ \\
\hline$C(213)$ & $C(214)$ & $C(215)$ & $122(2)$ & $C(411)$ & $C(416)$ & $C(415)$ & $117(3)$ \\
\hline$C(214)$ & $C(215)$ & $C(216)$ & $118(2)$ & $\mathrm{P}(4)$ & $C(421)$ & $C(422)$ & $123(2)$ \\
\hline$C(211)$ & $C(216)$ & $C(215)$ & $123(2)$ & $\mathrm{P}(4)$ & $C(421)$ & $C(426)$ & $116(2)$ \\
\hline $\mathrm{P}(3)$ & $C(311)$ & $C(312)$ & $122(2)$ & $C(422)$ & $C(421)$ & $C(426)$ & $121(2)$ \\
\hline$P(3)$ & $C(311)$ & $C(316)$ & $120(1)$ & $C(421)$ & $C(422)$ & $C(423)$ & $122(2)$ \\
\hline$C(312)$ & $C(311)$ & $C(316)$ & $117(2)$ & $C(422)$ & $C(423)$ & $C(424)$ & $119(2)$ \\
\hline$C(311)$ & $C(312)$ & $C(313)$ & $126(2)$ & $C(423)$ & $C(424)$ & $C(425)$ & $120(3)$ \\
\hline$C(312)$ & $C(313)$ & $C(314)$ & $115(2)$ & $C(424)$ & $C(425)$ & $C(426)$ & $123(2)$ \\
\hline$C(313)$ & $C(314)$ & $C(315)$ & $122(2)$ & $C(421)$ & $C(426)$ & $C(425)$ & $115(2)$ \\
\hline$C(314)$ & $C(315)$ & $C(316)$ & $120(2)$ & $P(5)$ & $C(511)$ & $C(512)$ & $118(2)$ \\
\hline$C(311)$ & $C(316)$ & $C(315)$ & $120(2)$ & $P(5)$ & $C(511)$ & $C(516)$ & $121(2)$ \\
\hline $\mathrm{P}(3)$ & $C(321)$ & $C(322)$ & $124(2)$ & $C(512)$ & $C(511)$ & $C(516)$ & $121(2)$ \\
\hline $\mathrm{P}(3)$ & $C(321)$ & $C(326)$ & $117(2)$ & $C(511)$ & $C(512)$ & $C(513)$ & $122(2)$ \\
\hline$C(322)$ & $C(321)$ & $C(326)$ & $119(2)$ & $C(512)$ & $C(513)$ & $C(514)$ & $115(2)$ \\
\hline$C(321)$ & $C(322)$ & $C(323)$ & $118(2)$ & $C(513)$ & $C(514)$ & $C(515)$ & $123(2)$ \\
\hline$C(322)$ & $C(323)$ & $C(324)$ & $123(2)$ & $C(514)$ & $C(515)$ & $C(516)$ & $120(2)$ \\
\hline$C(323)$ & $C(324)$ & $C(325)$ & $119(2)$ & $C(511)$ & $C(516)$ & $C(515)$ & $119(2)$ \\
\hline$C(324)$ & $C(325)$ & $C(326)$ & $119(2)$ & $P(6)$ & $C(611)$ & $C(612)$ & $121(2)$ \\
\hline$C(321)$ & $C(326)$ & $C(325)$ & $122(2)$ & $P(6)$ & $C(611)$ & $C(616)$ & $120(2)$ \\
\hline $\mathrm{P}(4)$ & $C(411)$ & $C(412)$ & $115(2)$ & $C(612)$ & $C(611)$ & $C(616)$ & $119(2)$ \\
\hline $\mathrm{P}(4)$ & $C(411)$ & $C(416)$ & $120(2)$ & $C(611)$ & $C(612)$ & $C(613)$ & $118(2)$ \\
\hline$C(412)$ & $C(411)$ & $C(416)$ & $125(2)$ & $C(612)$ & $C(613)$ & $C(614)$ & $124(2)$ \\
\hline$C(613)$ & $C(614)$ & $C(615)$ & $117(2)$ & & & & \\
\hline$C(614)$ & $C(615)$ & $C(616)$ & $123(2)$ & & & & \\
\hline$C(611)$ & $C(616)$ & $C(615)$ & $119(2)$ & & & & \\
\hline $\mathrm{P}(6)$ & $C(621)$ & $C(622)$ & $119(2)$ & & & & \\
\hline $\mathrm{P}(6)$ & $C(621)$ & $C(626)$ & $120(2)$ & & & & \\
\hline$C(622)$ & $C(621)$ & $C(626)$ & $121(2)$ & & & & \\
\hline$C(621)$ & $C(622)$ & $C(623)$ & $118(2)$ & & & & \\
\hline$C(622)$ & $C(623)$ & $C(624)$ & $122(2)$ & & & & \\
\hline$C(623)$ & $C(624)$ & $C(625)$ & $119(2)$ & & & & \\
\hline$C(624)$ & $C(625)$ & $C(626)$ & $123(2)$ & & & & \\
\hline$C(621)$ & $C(626)$ & $C(625)$ & $117(2)$ & & & & \\
\hline $\mathrm{O}(701)$ & $C(712)$ & $C(711)$ & $115(3)$ & & & & \\
\hline $\mathrm{O}(701)$ & $\mathrm{C}(713)$ & $C(714)$ & 111(3) & & & & \\
\hline $\mathrm{O}(702)$ & $C(724)$ & $C(721)$ & $120(3)$ & & & & \\
\hline $\mathrm{O}(702)$ & $C(724)$ & $C(723)$ & $121(3)$ & & & & \\
\hline$C(721)$ & $C(724)$ & $C(723)$ & $118(3)$ & & & & \\
\hline
\end{tabular}

a Estimated deviations are given in parentheses. 
Table S5-1. Crystallographic and Experimental Data for $\mathbf{6 c} \cdot 2 \mathrm{CH}_{3} \mathrm{CN}$

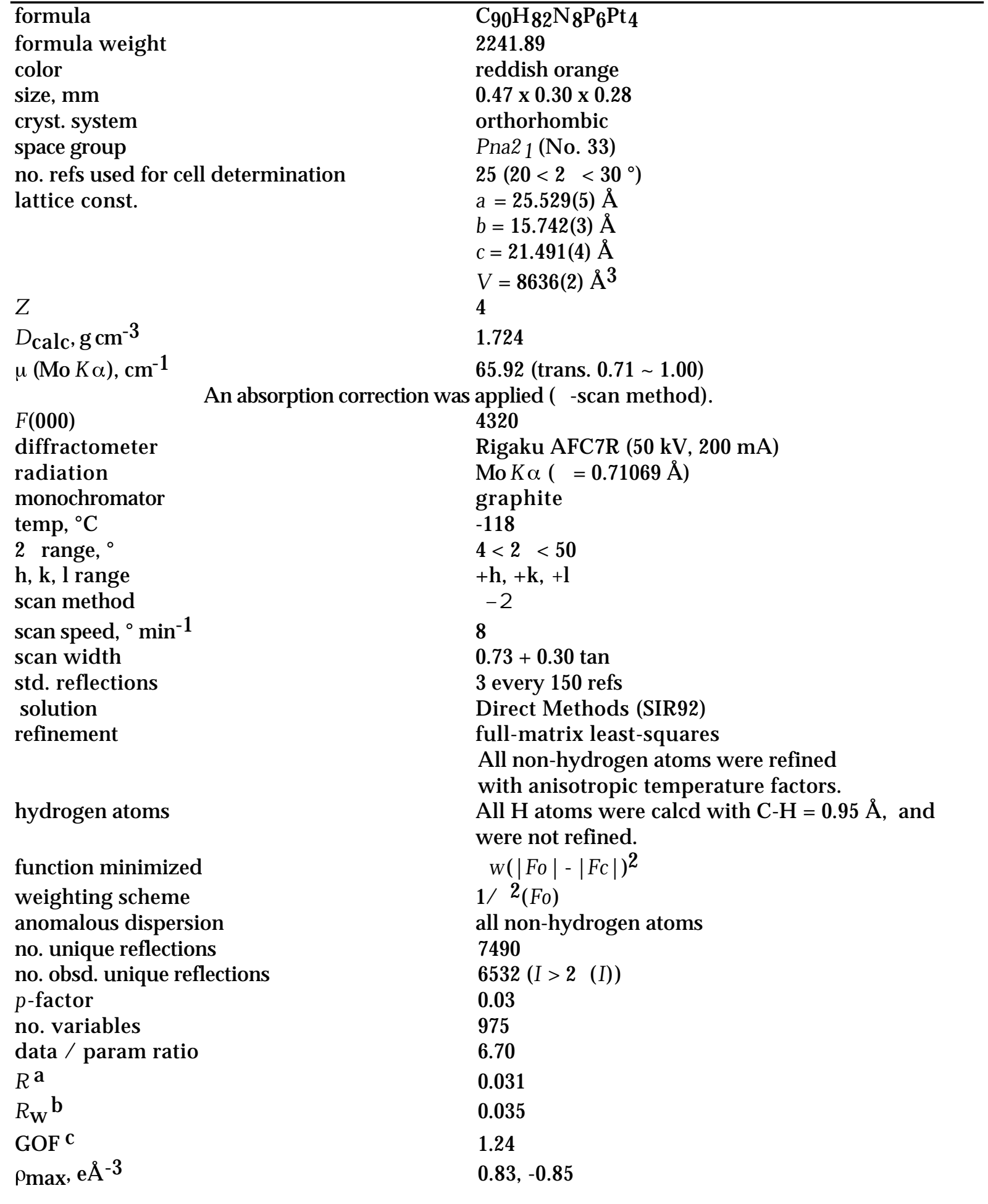

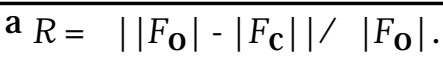

$\mathrm{b}_{R_{\mathrm{W}}}=\left[\Sigma w\left(\left|F_{\mathrm{O}}\right|-\left|F_{\mathrm{C}}\right|\right)^{2} / \Sigma w\left|F_{\mathrm{O}}\right|^{2}\right]^{1 / 2}\left(w=1 / \sigma^{2}\left(F_{\mathrm{O}}\right)\right)$.

${ }^{c} \mathrm{GOF}=\left[\Sigma w\left(\left|F_{\mathrm{O}}\right|-\left|F_{\mathrm{C}}\right|\right)^{2} /\left(N_{\mathrm{O}}-N_{\mathrm{p}}\right]^{1 / 2}\left(N_{\mathrm{O}}=\right.\right.$ no. data, $N_{\mathrm{p}}=$ no. variables $)$.

Table S5-2. Final Positional Parameters and $B$ eq for $6 c \cdot 2 \mathrm{CH}_{3} \mathrm{CN}^{\mathrm{a}-\mathrm{c}}$

\begin{tabular}{ccccc}
\hline atom & $x$ & $y$ & $z$ & $B_{\text {eq }}$ \\
\hline $\operatorname{Pt}(1)$ & $0.15997(2)$ & $-0.02617(3)$ & $0.9375(8)$ & $3.084(9)$ \\
$\operatorname{Pt}(2)$ & $0.22845(2)$ & $0.10194(3)$ & $0.9720(8)$ & $2.864(8)$
\end{tabular}




\begin{tabular}{|c|c|c|c|c|}
\hline $\operatorname{Pt}(3)$ & $0.29063(2)$ & $0.23481(3)$ & $1.0101(8)$ & 2.954(9) \\
\hline $\operatorname{Pt}(4)$ & $0.36880(2)$ & $-0.14248(3)$ & $1.2308(8)$ & $4.60(1)$ \\
\hline $\mathrm{P}(1)$ & $0.1577(1)$ & $0.0448(2)$ & $0.8452(8)$ & $3.31(6)$ \\
\hline $\mathrm{P}(2)$ & $0.1790(1)$ & $0.1959(2)$ & $0.9173(8)$ & $3.22(6)$ \\
\hline $\mathrm{P}(3)$ & $0.2813(1)$ & $0.2951(2)$ & $0.9134(8)$ & $3.17(6)$ \\
\hline $\mathrm{P}(4)$ & $0.1761(1)$ & $-0.0781(2)$ & $1.0345(8)$ & $3.08(6)$ \\
\hline $\mathrm{P}(5)$ & $0.2772(1)$ & $0.0193(2)$ & $1.0361(8)$ & $2.90(6)$ \\
\hline $\mathrm{P}(6)$ & $0.2991(1)$ & $0.1735(2)$ & $1.1066(8)$ & $3.25(6)$ \\
\hline $\mathrm{N}(1)$ & $0.0691(4)$ & $-0.1543(6)$ & $0.8991(9)$ & $3.9(2)$ \\
\hline $\mathrm{N}(2)$ & $0.3666(4)$ & $0.3896(6)$ & $1.0396(9)$ & $3.8(2)$ \\
\hline $\mathrm{N}(3)$ & $0.4434(7)$ & $-0.0445(9)$ & $1.3254(9)$ & $7.9(4)$ \\
\hline $\mathrm{N}(4)$ & $0.4693(5)$ & $-0.2485(8)$ & $1.1852(10)$ & $6.1(3)$ \\
\hline $\mathrm{N}(5)$ & $0.3043(5)$ & $-0.2449(6)$ & $1.1306(9)$ & $4.7(3)$ \\
\hline $\mathrm{N}(6)$ & $0.2651(6)$ & $-0.0316(8)$ & $1.2619(9)$ & $6.2(3)$ \\
\hline $\mathrm{N}(701)$ & $0.6894(6)$ & $0.183(1)$ & $0.4169(10)$ & $7.6(4)$ \\
\hline $\mathrm{N}(702)$ & $0.0985(8)$ & $0.435(1)$ & $0.198(1)$ & $12.7(7)$ \\
\hline$C(1)$ & $0.1035(5)$ & $-0.1097(7)$ & $0.9151(9)$ & $3.9(3)$ \\
\hline$C(2)$ & $0.3373(5)$ & $0.3323(7)$ & $1.0318(9)$ & $3.3(2)$ \\
\hline$C(3)$ & $0.4146(7)$ & $-0.0777(9)$ & $1.292(1)$ & $6.1(4)$ \\
\hline$C(4)$ & $0.4344(6)$ & $-0.2133(9)$ & $1.205(1)$ & $4.8(3)$ \\
\hline$C(5)$ & $0.3254(6)$ & $-0.2083(8)$ & $1.1679(10)$ & $4.3(3)$ \\
\hline$C(6)$ & $0.3038(7)$ & $-0.0719(8)$ & $1.2515(10)$ & $5.4(3)$ \\
\hline$C(7)$ & $0.1315(5)$ & $0.1510(7)$ & $0.8596(9)$ & $3.4(2)$ \\
\hline$C(8)$ & $0.2222(4)$ & $0.2622(7)$ & $0.8685(9)$ & $3.4(2)$ \\
\hline$C(9)$ & $0.2488(5)$ & $-0.0874(7)$ & $1.0477(9)$ & $3.3(2)$ \\
\hline$C(10)$ & $0.2737(4)$ & $0.0643(7)$ & $1.1127(9)$ & $2.9(2)$ \\
\hline$C(11)$ & $0.0255(5)$ & $-0.2059(8)$ & $0.8787(9)$ & $3.9(2)$ \\
\hline$C(12)$ & $-0.0226(5)$ & $-0.1690(8)$ & $0.8608(9)$ & $4.2(2)$ \\
\hline$C(13)$ & $-0.0635(5)$ & $-0.2248(10)$ & $0.8396(10)$ & $4.7(3)$ \\
\hline$C(14)$ & $-0.0566(6)$ & $-0.3104(9)$ & $0.8363(9)$ & $4.8(3)$ \\
\hline$C(15)$ & $-0.0060(5)$ & $-0.3442(8)$ & $0.8547(10)$ & $4.7(3)$ \\
\hline$C(16)$ & $0.0358(5)$ & $-0.2946(8)$ & $0.8769(9)$ & $4.1(2)$ \\
\hline$C(17)$ & $-0.0309(5)$ & $-0.0762(9)$ & $0.8640(10)$ & $4.8(3)$ \\
\hline$C(18)$ & $0.0891(6)$ & $-0.3312(10)$ & $0.896(1)$ & $6.5(4)$ \\
\hline$C(21)$ & $0.3994(5)$ & $0.4606(7)$ & $1.0495(9)$ & $3.7(2)$ \\
\hline$C(22)$ & $0.3748(5)$ & $0.5287(8)$ & $1.0793(9)$ & $3.9(2)$ \\
\hline$C(23)$ & $0.4084(5)$ & $0.5982(8)$ & $1.0920(10)$ & $4.5(3)$ \\
\hline$C(24)$ & $0.4612(6)$ & $0.5999(9)$ & $1.073(1)$ & $5.4(3)$ \\
\hline$C(25)$ & $0.4834(5)$ & $0.5307(9)$ & $1.041(1)$ & $5.0(3)$ \\
\hline$C(26)$ & $0.4529(5)$ & $0.4604(8)$ & $1.0293(9)$ & $4.1(2)$ \\
\hline$C(27)$ & $0.3157(6)$ & $0.5271(8)$ & $1.0990(10)$ & $4.4(3)$ \\
\hline$C(28)$ & $0.4766(6)$ & $0.3853(8)$ & $0.997(1)$ & $5.4(4)$ \\
\hline$C(111)$ & $0.1138(5)$ & $0.0010(8)$ & $0.7846(9)$ & $3.7(2)$ \\
\hline$C(112)$ & $0.0679(5)$ & $0.0429(8)$ & $0.7627(10)$ & $4.5(3)$ \\
\hline$C(113)$ & $0.0352(6)$ & $0.0011(10)$ & $0.7175(10)$ & $5.3(3)$ \\
\hline$C(114)$ & $0.0467(5)$ & $-0.0796(9)$ & $0.6996(10)$ & $5.1(3)$ \\
\hline$C(115)$ & $0.0909(6)$ & $-0.1211(8)$ & $0.7206(9)$ & $4.7(3)$ \\
\hline$C(116)$ & $0.1246(5)$ & $-0.0818(8)$ & $0.7642(10)$ & $4.3(3)$ \\
\hline$C(121)$ & $0.2224(5)$ & $0.0621(7)$ & $0.8054(9)$ & $3.4(2)$ \\
\hline$C(122)$ & $0.2709(5)$ & $0.0319(8)$ & $0.8288(9)$ & $3.9(2)$ \\
\hline$C(123)$ & $0.3187(5)$ & 0.0493(8) & $0.8011(10)$ & $4.6(3)$ \\
\hline$C(124)$ & $0.3197(5)$ & $0.0977(8)$ & $0.7473(9)$ & $4.5(3)$ \\
\hline$C(125)$ & $0.2716(5)$ & $0.1283(9)$ & $0.7214(9)$ & $4.5(3)$ \\
\hline$C(126)$ & $0.2225(5)$ & $0.1080(7)$ & $0.7496(9)$ & $3.7(2)$ \\
\hline$C(211)$ & $0.1388(5)$ & $0.2682(7)$ & $0.9618(9)$ & $3.5(2)$ \\
\hline$C(212)$ & $0.1487(5)$ & $0.2780(8)$ & $1.0278(9)$ & $3.7(3)$ \\
\hline$C(213)$ & $0.1195(6)$ & $0.3371(9)$ & $1.0605(9)$ & $4.8(3)$ \\
\hline$C(214)$ & $0.0811(6)$ & $0.3876(9)$ & $1.032(1)$ & $5.3(3)$ \\
\hline
\end{tabular}




\begin{tabular}{|c|c|c|c|c|}
\hline$C(215)$ & $0.0708(5)$ & $0.3793(8)$ & $0.969(1)$ & $4.9(3)$ \\
\hline$C(216)$ & $0.0988(5)$ & $0.3193(7)$ & $0.9344(9)$ & 4.1(3) \\
\hline$C(311)$ & $0.2750(4)$ & $0.4116(7)$ & $0.9168(9)$ & $3.1(2)$ \\
\hline$C(312)$ & $0.3183(5)$ & $0.4641(7)$ & $0.9071(10)$ & $4.1(3)$ \\
\hline C(313) & $0.3127(6)$ & $0.5519(8)$ & $0.912(1)$ & $5.0(3)$ \\
\hline C(314) & $0.2628(6)$ & $0.5869(8)$ & $0.924(1)$ & $5.2(3)$ \\
\hline$C(315)$ & $0.2188(6)$ & $0.5344(8)$ & $0.933(1)$ & $5.1(3)$ \\
\hline$C(316)$ & $0.2244(5)$ & $0.4474(8)$ & $0.929(1)$ & $4.2(2)$ \\
\hline$C(321)$ & $0.3412(5)$ & $0.2762(7)$ & $0.8654(9)$ & $3.4(2)$ \\
\hline$C(322)$ & $0.3852(5)$ & $0.2334(8)$ & $0.8890(9)$ & $4.0(3)$ \\
\hline$C(323)$ & $0.4320(6)$ & $0.223(1)$ & $0.854(1)$ & $5.5(3)$ \\
\hline C(324) & $0.4352(6)$ & $0.2562(10)$ & $0.794(1)$ & $5.9(3)$ \\
\hline C(325) & $0.3889(7)$ & $0.2973(10)$ & $0.7701(10)$ & $5.9(4)$ \\
\hline C(326) & $0.3429(5)$ & $0.3099(8)$ & $0.8045(10)$ & $4.4(3)$ \\
\hline$C(411)$ & $0.1471(5)$ & $-0.0138(7)$ & $1.0977(9)$ & $3.4(2)$ \\
\hline$C(412)$ & $0.1186(5)$ & $0.0602(7)$ & $1.0827(9)$ & $3.6(3)$ \\
\hline$C(413)$ & $0.1001(6)$ & $0.1118(8)$ & $1.1308(10)$ & $4.6(3)$ \\
\hline$C(414)$ & $0.1089(6)$ & $0.0885(9)$ & $1.1909(10)$ & $5.4(3)$ \\
\hline C(415) & $0.1367(6)$ & $0.0172(9)$ & $1.2063(10)$ & $5.4(3)$ \\
\hline$C(416)$ & $0.1554(5)$ & $-0.0361(9)$ & $1.1598(10)$ & $4.5(3)$ \\
\hline$C(421)$ & $0.1510(5)$ & $-0.1847(7)$ & $1.0515(9)$ & $3.4(2)$ \\
\hline$C(422)$ & $0.0981(6)$ & $-0.1972(9)$ & $1.071(1)$ & $6.4(4)$ \\
\hline$C(423)$ & $0.0774(7)$ & $-0.2758(9)$ & $1.082(1)$ & $7.5(5)$ \\
\hline$C(424)$ & $0.1109(6)$ & $-0.3475(8)$ & $1.077(1)$ & $5.3(3)$ \\
\hline$C(425)$ & $0.1644(6)$ & $-0.3365(8)$ & $1.0613(10)$ & $4.5(3)$ \\
\hline$C(426)$ & $0.1850(5)$ & $-0.2562(8)$ & $1.0459(10)$ & $4.3(3)$ \\
\hline$C(511)$ & $0.3493(4)$ & $0.0013(7)$ & $1.0221(9)$ & $3.3(2)$ \\
\hline$C(512)$ & $0.3735(5)$ & $0.0268(7)$ & $0.9679(9)$ & $3.8(2)$ \\
\hline$C(513)$ & $0.4271(5)$ & $0.0081(8)$ & $0.9545(9)$ & $4.3(3)$ \\
\hline$C(514)$ & $0.4585(5)$ & $-0.0318(8)$ & $0.998(1)$ & $4.4(3)$ \\
\hline C(515) & $0.4362(5)$ & $-0.0580(8)$ & $1.0538(10)$ & $4.5(3)$ \\
\hline$C(516)$ & $0.3821(5)$ & $-0.0407(8)$ & $1.0670(9)$ & $4.2(3)$ \\
\hline$C(611)$ & $0.3692(5)$ & $0.1659(7)$ & $1.1325(9)$ & $3.6(2)$ \\
\hline C(612) & $0.4127(5)$ & $0.1923(8)$ & $1.0949(9)$ & $4.2(3)$ \\
\hline$C(613)$ & $0.4660(5)$ & $0.1888(9)$ & $1.118(1)$ & $5.4(3)$ \\
\hline$C(614)$ & $0.4764(6)$ & $0.1644(10)$ & $1.178(1)$ & $6.2(4)$ \\
\hline C(615) & $0.4336(6)$ & $0.136(1)$ & $1.215(1)$ & $6.0(3)$ \\
\hline C(616) & $0.3808(6)$ & $0.1378(9)$ & $1.1924(10)$ & $5.2(3)$ \\
\hline$C(621)$ & $0.2621(5)$ & $0.2306(7)$ & $1.1676(9)$ & $3.4(2)$ \\
\hline$C(622)$ & $0.2473(5)$ & $0.1902(7)$ & $1.2225(9)$ & $3.9(3)$ \\
\hline$C(623)$ & $0.2222(5)$ & $0.2365(8)$ & $1.2692(9)$ & $4.3(3)$ \\
\hline$C(624)$ & $0.2131(6)$ & $0.3217(9)$ & $1.2625(10)$ & $5.0(3)$ \\
\hline C(625) & $0.2269(6)$ & $0.3608(8)$ & $1.2083(10)$ & $4.7(3)$ \\
\hline C(626) & $0.2521(5)$ & $0.3162(8)$ & $1.1605(9)$ & $4.1(3)$ \\
\hline$C(701)$ & $0.6577(7)$ & $0.2098(10)$ & $0.450(1)$ & $5.8(4)$ \\
\hline$C(702)$ & $0.6184(8)$ & $0.244(1)$ & $0.490(1)$ & $7.7(5)$ \\
\hline C(703) & $0.0679(8)$ & $0.488(1)$ & $0.203(1)$ & $7.5(5)$ \\
\hline$C(704)$ & $0.0286(9)$ & $0.554(1)$ & $0.208(2)$ & $11.8(8)$ \\
\hline $\mathrm{H}(1)$ & 0.1287 & 0.1856 & 0.8227 & 4.5 \\
\hline $\mathrm{H}(2)$ & 0.0957 & 0.1494 & 0.8772 & 4.5 \\
\hline $\mathrm{H}(3)$ & 0.2331 & 0.2291 & 0.8320 & 4.1 \\
\hline $\mathrm{H}(4)$ & 0.2035 & 0.3102 & 0.8531 & 4.1 \\
\hline $\mathrm{H}(5)$ & 0.2558 & -0.1077 & 1.0901 & 4.1 \\
\hline $\mathrm{H}(6)$ & 0.2643 & -0.1284 & 1.0203 & 4.1 \\
\hline $\mathrm{H}(7)$ & 0.2384 & 0.0660 & 1.1281 & 3.7 \\
\hline $\mathrm{H}(8)$ & 0.2944 & 0.0331 & 1.1423 & 3.7 \\
\hline $\mathrm{H}(9)$ & -0.0985 & -0.1998 & 0.8275 & 6.0 \\
\hline $\mathrm{H}(10)$ & -0.0851 & -0.3474 & 0.8232 & 5.7 \\
\hline
\end{tabular}




\begin{tabular}{|c|c|c|c|c|}
\hline $\mathrm{H}(11)$ & -0.0011 & -0.4055 & 0.8519 & 5.5 \\
\hline $\mathrm{H}(12)$ & 0.3940 & 0.6477 & 1.1151 & 5.5 \\
\hline $\mathrm{H}(13)$ & 0.4825 & 0.6494 & 1.0814 & 6.3 \\
\hline $\mathrm{H}(14)$ & 0.5209 & 0.5345 & 1.0283 & 5.9 \\
\hline $\mathrm{H}(15)$ & 0.0589 & 0.1001 & 0.7763 & 5.4 \\
\hline $\mathrm{H}(16)$ & 0.0056 & 0.0330 & 0.6988 & 6.4 \\
\hline $\mathrm{H}(17)$ & 0.0212 & -0.1104 & 0.6735 & 6.1 \\
\hline $\mathrm{H}(18)$ & 0.0995 & -0.1763 & 0.7050 & 5.5 \\
\hline $\mathrm{H}(19)$ & 0.1551 & -0.1145 & 0.7802 & 5.3 \\
\hline $\mathrm{H}(20)$ & 0.2706 & 0.0003 & 0.8673 & 4.4 \\
\hline $\mathrm{H}(21)$ & 0.3512 & 0.0267 & 0.8182 & 5.2 \\
\hline $\mathrm{H}(22)$ & 0.3527 & 0.1144 & 0.7298 & 5.0 \\
\hline $\mathrm{H}(23)$ & 0.2711 & 0.1578 & 0.6825 & 5.1 \\
\hline $\mathrm{H}(24)$ & 0.1906 & 0.1304 & 0.7338 & 4.4 \\
\hline $\mathrm{H}(25)$ & 0.1751 & 0.2440 & 1.0494 & 4.8 \\
\hline $\mathrm{H}(26)$ & 0.1230 & 0.3409 & 1.1051 & 5.7 \\
\hline $\mathrm{H}(27)$ & 0.0616 & 0.4272 & 1.0560 & 6.4 \\
\hline $\mathrm{H}(28)$ & 0.0452 & 0.4184 & 0.9503 & 5.6 \\
\hline $\mathrm{H}(29)$ & 0.0915 & 0.3138 & 0.8900 & 4.7 \\
\hline $\mathrm{H}(30)$ & 0.3524 & 0.4404 & 0.8964 & 5.0 \\
\hline $\mathrm{H}(31)$ & 0.3428 & 0.5883 & 0.9071 & 6.2 \\
\hline $\mathrm{H}(32)$ & 0.2580 & 0.6489 & 0.9252 & 6.3 \\
\hline $\mathrm{H}(33)$ & 0.1851 & 0.5580 & 0.9432 & 5.8 \\
\hline $\mathrm{H}(34)$ & 0.1943 & 0.4112 & 0.9362 & 4.6 \\
\hline $\mathrm{H}(35)$ & 0.3844 & 0.2083 & 0.9295 & 5.0 \\
\hline $\mathrm{H}(36)$ & 0.4609 & 0.1917 & 0.8716 & 6.6 \\
\hline $\mathrm{H}(37)$ & 0.4691 & 0.2521 & 0.7715 & 7.1 \\
\hline $\mathrm{H}(38)$ & 0.3895 & 0.3174 & 0.7286 & 6.6 \\
\hline $\mathrm{H}(39)$ & 0.3136 & 0.3409 & 0.7872 & 5.2 \\
\hline $\mathrm{H}(40)$ & 0.1120 & 0.0762 & 1.0394 & 4.4 \\
\hline $\mathrm{H}(41)$ & 0.0829 & 0.1654 & 1.1230 & 5.5 \\
\hline $\mathrm{H}(42)$ & 0.0919 & 0.1242 & 1.2227 & 6.2 \\
\hline $\mathrm{H}(43)$ & 0.1455 & 0.0068 & 1.2487 & 6.8 \\
\hline $\mathrm{H}(44)$ & 0.1727 & -0.0876 & 1.1703 & 5.7 \\
\hline $\mathrm{H}(45)$ & 0.0744 & -0.1469 & 1.0746 & 7.4 \\
\hline $\mathrm{H}(46)$ & 0.0429 & -0.2833 & 1.0994 & 9.0 \\
\hline $\mathrm{H}(47)$ & 0.0961 & -0.4043 & 1.0816 & 6.2 \\
\hline $\mathrm{H}(48)$ & 0.1870 & -0.3867 & 1.0608 & 5.6 \\
\hline $\mathrm{H}(49)$ & 0.2212 & -0.2494 & 1.0336 & 5.2 \\
\hline $\mathrm{H}(50)$ & 0.3534 & 0.0609 & 0.9380 & 4.7 \\
\hline $\mathrm{H}(51)$ & 0.4420 & 0.0259 & 0.9154 & 5.1 \\
\hline $\mathrm{H}(52)$ & 0.4958 & -0.0432 & 0.9898 & 5.4 \\
\hline $\mathrm{H}(53)$ & 0.4580 & -0.0910 & 1.0842 & 5.1 \\
\hline $\mathrm{H}(54)$ & 0.3684 & -0.0553 & 1.1080 & 4.9 \\
\hline $\mathrm{H}(55)$ & 0.4060 & 0.2116 & 1.0531 & 4.9 \\
\hline $\mathrm{H}(56)$ & 0.4952 & 0.2029 & 1.0909 & 6.5 \\
\hline $\mathrm{H}(57)$ & 0.5123 & 0.1678 & 1.1935 & 7.5 \\
\hline $\mathrm{H}(58)$ & 0.4412 & 0.1137 & 1.2560 & 6.6 \\
\hline H(59) & 0.3515 & 0.1192 & 1.2198 & 6.2 \\
\hline $\mathrm{H}(60)$ & 0.2538 & 0.1282 & 1.2290 & 4.5 \\
\hline $\mathrm{H}(61)$ & 0.2121 & 0.2086 & 1.3077 & 5.1 \\
\hline $\mathrm{H}(62)$ & 0.1955 & 0.3518 & 1.2964 & 5.8 \\
\hline $\mathrm{H}(63)$ & 0.2188 & 0.4212 & 1.2045 & 5.7 \\
\hline $\mathrm{H}(64)$ & 0.2623 & 0.3460 & 1.1219 & 4.9 \\
\hline $\mathrm{H}(65)$ & -0.0257 & -0.0548 & 0.9055 & 5.8 \\
\hline $\mathrm{H}(66)$ & -0.0054 & -0.0468 & 0.8377 & 5.8 \\
\hline $\mathrm{H}(67)$ & -0.0647 & -0.0599 & 0.8502 & 5.8 \\
\hline $\mathrm{H}(68)$ & 0.0903 & -0.3915 & 0.8939 & 6.8 \\
\hline
\end{tabular}




$\begin{array}{lrrrr}\mathrm{H}(69) & 0.1170 & -0.3085 & 0.8712 & 6.8 \\ \mathrm{H}(70) & 0.0977 & -0.3160 & 0.9394 & 6.8 \\ \mathrm{H}(71) & 0.3061 & 0.5809 & 1.1184 & 5.6 \\ \mathrm{H}(72) & 0.2936 & 0.5200 & 1.0637 & 5.6 \\ \mathrm{H}(73) & 0.3094 & 0.4834 & 1.1281 & 5.6 \\ \mathrm{H}(74) & 0.4527 & 0.3410 & 0.9907 & 6.3 \\ \mathrm{H}(75) & 0.4909 & 0.4021 & 0.9568 & 6.3 \\ \mathrm{H}(76) & 0.5062 & 0.3636 & 1.0208 & 6.3 \\ \mathrm{H}(77) & 0.6293 & 0.2920 & 0.5138 & 8.7 \\ \mathrm{H}(78) & 0.5874 & 0.2630 & 0.4660 & 8.7 \\ \mathrm{H}(79) & 0.6047 & 0.2022 & 0.2346 & 15.3 \\ \mathrm{H}(80) & 0.0362 & 0.5975 & 0.1648 & 15.3 \\ \mathrm{H}(81) & 0.0228 & 0.5856 & 0.2148 & 15.3 \\ \mathrm{H}(82) & -0.0069 & 0.5335 & \end{array}$

a Estimated standard deviations are given in parentheses. ${ }^{b}$ All non-hydrogen atoms were assigned anisotropic thermal parameters given as the isotropic equivalent displacement parameter defined as $B_{\text {eq }}=\left(8 \pi^{2} / 3\right)\left(U_{11}\left(a a^{*}\right)^{2}+U_{22}\left(b b^{*}\right)^{2}+U_{33}\left(c c^{*}\right)^{2}+2 U_{12} a a^{*} b b^{*} \cos \gamma+2 U_{13} a a^{*} c c^{*} \cos \beta+\right.$ $\left.2 U_{23} b b^{*} c c^{*} \cos \alpha\right)$. ${ }^{c}$ The positions of hydrogen atoms were calculated with $\mathrm{C}-\mathrm{H}=0.95 \AA$, and were not refined with appropriate $B$ iso.

Table S5-3. Final Anisotropic Thermal Parameters for $6 \mathrm{c} \cdot 2 \mathrm{CH}_{3} \mathrm{CN}^{\mathrm{a}}, \mathrm{b}$

\begin{tabular}{|c|c|c|c|c|c|c|}
\hline atom & $U_{11}$ & $U_{22}$ & $U_{33}$ & $U_{12}$ & $U_{13}$ & $U_{23}$ \\
\hline$\overline{\operatorname{Pt}(1)}$ & $0.0533(3)$ & $0.0388(2)$ & $0.0251(2)$ & $-0.0023(2)$ & $-0.0024(2)$ & $0.0019(2)$ \\
\hline $\operatorname{Pt}(2)$ & $0.0506(2)$ & $0.0365(2)$ & $0.0217(2)$ & $-0.0010(2)$ & $0.0000(2)$ & $0.0016(2)$ \\
\hline $\operatorname{Pt}(3)$ & $0.0524(3)$ & $0.0379(2)$ & $0.0219(2)$ & $-0.0019(2)$ & $0.0011(2)$ & $0.0008(2)$ \\
\hline $\operatorname{Pt}(4)$ & $0.0896(4)$ & $0.0500(3)$ & $0.0352(3)$ & $-0.0093(3)$ & $-0.0049(3)$ & $0.0107(2)$ \\
\hline $\mathrm{P}(1)$ & $0.060(2)$ & $0.042(2)$ & $0.024(1)$ & $-0.003(2)$ & $-0.002(1)$ & $0.002(1)$ \\
\hline$P(2)$ & $0.057(2)$ & $0.040(2)$ & $0.026(1)$ & $-0.001(1)$ & $-0.001(1)$ & $0.002(1)$ \\
\hline $\mathrm{P}(3)$ & $0.055(2)$ & $0.041(2)$ & $0.024(1)$ & $-0.002(1)$ & $0.003(1)$ & $0.002(1)$ \\
\hline $\mathrm{P}(4)$ & $0.053(2)$ & $0.040(2)$ & $0.024(1)$ & $-0.002(1)$ & $0.001(1)$ & $0.002(1)$ \\
\hline$P(5)$ & $0.050(2)$ & $0.036(2)$ & $0.024(1)$ & $-0.004(1)$ & $-0.002(1)$ & $0.003(1)$ \\
\hline$P(6)$ & $0.059(2)$ & $0.043(2)$ & $0.022(1)$ & $-0.002(2)$ & $0.001(1)$ & $0.002(1)$ \\
\hline $\mathrm{N}(1)$ & $0.067(6)$ & $0.050(6)$ & $0.029(5)$ & $-0.008(4)$ & $-0.003(5)$ & $0.002(4)$ \\
\hline $\mathrm{N}(2)$ & $0.061(7)$ & $0.049(6)$ & $0.035(5)$ & $-0.014(4)$ & $-0.005(5)$ & $0.001(5)$ \\
\hline $\mathrm{N}(3)$ & $0.15(1)$ & $0.084(10)$ & $0.059(8)$ & $-0.060(9)$ & $-0.040(8)$ & $0.000(7)$ \\
\hline $\mathrm{N}(4)$ & $0.074(8)$ & $0.087(10)$ & $0.072(9)$ & $-0.002(6)$ & $-0.022(7)$ & $-0.008(7)$ \\
\hline $\mathrm{N}(5)$ & $0.083(8)$ & $0.045(6)$ & $0.052(6)$ & $0.003(6)$ & $-0.021(5)$ & $0.007(5)$ \\
\hline $\mathrm{N}(6)$ & $0.115(9)$ & $0.073(9)$ & $0.048(7)$ & $0.023(7)$ & $0.015(7)$ & $0.001(6)$ \\
\hline $\mathrm{N}(701)$ & $0.12(1)$ & $0.13(1)$ & $0.045(8)$ & $0.010(9)$ & $-0.004(6)$ & $0.034(8)$ \\
\hline $\mathrm{N}(702)$ & $0.11(1)$ & $0.12(1)$ & $0.25(3)$ & $-0.001(9)$ & $0.02(2)$ & $-0.06(2)$ \\
\hline$C(1)$ & $0.074(8)$ & $0.039(6)$ & $0.034(6)$ & $-0.007(5)$ & $-0.006(6)$ & $-0.012(5)$ \\
\hline$C(2)$ & $0.060(8)$ & $0.045(6)$ & $0.021(5)$ & $-0.008(4)$ & $-0.001(5)$ & $0.006(5)$ \\
\hline$C(3)$ & $0.13(1)$ & $0.055(9)$ & $0.048(8)$ & $-0.020(8)$ & $-0.023(8)$ & $0.001(6)$ \\
\hline$C(4)$ & $0.065(8)$ & $0.062(9)$ & $0.057(9)$ & $-0.023(5)$ & $-0.028(6)$ & $0.013(7)$ \\
\hline$C(5)$ & $0.077(9)$ & $0.043(7)$ & $0.044(7)$ & $-0.003(6)$ & $-0.012(6)$ & $0.018(5)$ \\
\hline$C(6)$ & $0.104(10)$ & $0.047(8)$ & $0.054(9)$ & $0.000(6)$ & $0.005(8)$ & $0.013(7)$ \\
\hline$C(7)$ & $0.059(7)$ & $0.044(5)$ & $0.028(6)$ & $0.003(5)$ & $-0.008(4)$ & $0.000(4)$ \\
\hline$C(8)$ & $0.042(5)$ & $0.048(7)$ & $0.039(6)$ & $0.004(4)$ & $0.002(3)$ & $0.013(5)$ \\
\hline$C(9)$ & $0.052(3)$ & $0.034(4)$ & $0.038(6)$ & $-0.001(5)$ & $-0.004(6)$ & $0.012(5)$ \\
\hline$C(10)$ & $0.046(6)$ & $0.041(4)$ & $0.024(4)$ & $0.006(5)$ & $0.000(5)$ & $0.000(4)$ \\
\hline$C(11)$ & $0.062(6)$ & $0.057(5)$ & $0.028(6)$ & $-0.007(4)$ & $0.005(5)$ & $-0.003(5)$ \\
\hline$C(12)$ & $0.066(6)$ & $0.066(6)$ & $0.029(6)$ & $0.000(5)$ & $0.005(5)$ & $0.007(6)$ \\
\hline$C(13)$ & $0.061(8)$ & $0.081(7)$ & $0.037(7)$ & $0.003(6)$ & $-0.005(6)$ & $-0.005(7)$ \\
\hline$C(14)$ & $0.067(7)$ & $0.077(7)$ & $0.039(7)$ & $-0.029(7)$ & $0.017(6)$ & $-0.013(7)$ \\
\hline
\end{tabular}




\begin{tabular}{|c|c|c|c|c|c|c|}
\hline$C(15)$ & $0.076(7)$ & $0.059(8)$ & $0.041(7)$ & $-0.025(6)$ & $0.007(6)$ & $0.004(6)$ \\
\hline$C(16)$ & $0.068(6)$ & $0.058(5)$ & $0.029(6)$ & $-0.005(5)$ & $0.010(5)$ & $-0.011(5)$ \\
\hline$C(17)$ & $0.055(8)$ & $0.067(6)$ & $0.059(8)$ & $0.003(7)$ & $-0.001(7)$ & $0.004(7)$ \\
\hline$C(18)$ & $0.073(9)$ & $0.072(10)$ & $0.10(1)$ & $-0.011(8)$ & $-0.036(9)$ & $-0.001(9)$ \\
\hline$C(21)$ & $0.059(6)$ & $0.044(5)$ & $0.036(6)$ & $-0.006(4)$ & $-0.014(5)$ & $0.009(5)$ \\
\hline$C(22)$ & $0.071(6)$ & $0.048(6)$ & $0.031(6)$ & $-0.007(5)$ & $-0.003(5)$ & $0.003(5)$ \\
\hline$C(23)$ & $0.071(7)$ & $0.046(7)$ & $0.055(8)$ & $-0.002(6)$ & $-0.006(6)$ & $-0.010(6)$ \\
\hline$C(24)$ & $0.069(7)$ & $0.054(8)$ & $0.08(1)$ & $-0.019(7)$ & $-0.004(7)$ & $-0.009(7)$ \\
\hline$C(25)$ & $0.058(8)$ & $0.063(7)$ & $0.071(9)$ & $-0.012(6)$ & $0.003(7)$ & $0.000(7)$ \\
\hline$C(26)$ & $0.060(6)$ & $0.059(6)$ & $0.035(6)$ & $-0.007(5)$ & $-0.009(5)$ & $0.013(5)$ \\
\hline$C(27)$ & $0.072(7)$ & $0.051(7)$ & $0.046(7)$ & $-0.001(7)$ & $0.008(7)$ & $-0.006(6)$ \\
\hline$C(28)$ & $0.079(10)$ & $0.057(7)$ & $0.068(10)$ & $-0.001(7)$ & $0.009(8)$ & $0.006(6)$ \\
\hline$C(111)$ & $0.067(7)$ & $0.050(5)$ & $0.023(5)$ & $-0.021(5)$ & $-0.002(4)$ & $0.005(4)$ \\
\hline$C(112)$ & $0.075(8)$ & $0.053(8)$ & $0.042(7)$ & $-0.006(6)$ & $-0.011(6)$ & $0.006(6)$ \\
\hline$C(113)$ & $0.076(9)$ & $0.081(8)$ & $0.046(8)$ & $-0.014(8)$ & $-0.021(7)$ & $0.002(7)$ \\
\hline$C(114)$ & $0.065(8)$ & $0.081(8)$ & $0.048(8)$ & $-0.036(6)$ & $-0.002(6)$ & $-0.012(7)$ \\
\hline$C(115)$ & $0.079(8)$ & $0.061(8)$ & $0.040(7)$ & $-0.018(6)$ & $0.008(5)$ & $-0.013(6)$ \\
\hline$C(116)$ & $0.064(8)$ & $0.054(6)$ & $0.044(7)$ & $0.002(6)$ & $-0.005(6)$ & $0.003(5)$ \\
\hline$C(121)$ & $0.063(4)$ & $0.042(6)$ & $0.026(5)$ & $-0.003(5)$ & $0.005(4)$ & $-0.002(4)$ \\
\hline$C(122)$ & $0.069(6)$ & $0.055(7)$ & $0.024(6)$ & $0.014(6)$ & $0.003(4)$ & $-0.009(5)$ \\
\hline$C(123)$ & $0.071(7)$ & $0.057(8)$ & $0.047(6)$ & $-0.001(7)$ & $0.008(6)$ & $-0.011(5)$ \\
\hline$C(124)$ & $0.071(8)$ & $0.061(8)$ & $0.040(6)$ & $-0.007(7)$ & $0.002(6)$ & $-0.019(5)$ \\
\hline$C(125)$ & $0.070(6)$ & $0.067(9)$ & $0.036(7)$ & $-0.003(6)$ & $0.012(5)$ & $-0.011(6)$ \\
\hline$C(126)$ & $0.069(7)$ & $0.045(7)$ & $0.028(5)$ & $-0.006(6)$ & $-0.002(5)$ & $0.003(4)$ \\
\hline$C(211)$ & $0.061(7)$ & $0.046(6)$ & $0.027(5)$ & $-0.002(4)$ & $-0.002(4)$ & $-0.006(4)$ \\
\hline$C(212)$ & $0.059(8)$ & $0.057(7)$ & $0.025(5)$ & $0.007(6)$ & $-0.005(5)$ & $0.005(5)$ \\
\hline$C(213)$ & $0.077(9)$ & $0.064(8)$ & $0.041(7)$ & $0.001(6)$ & $0.018(6)$ & $-0.015(6)$ \\
\hline$C(214)$ & $0.081(10)$ & $0.067(9)$ & $0.053(6)$ & $0.003(7)$ & $0.013(6)$ & $-0.007(7)$ \\
\hline$C(215)$ & $0.075(9)$ & $0.061(8)$ & $0.051(5)$ & $0.023(7)$ & $0.010(7)$ & $0.009(6)$ \\
\hline$C(216)$ & $0.069(8)$ & $0.055(7)$ & $0.031(6)$ & $0.004(5)$ & $-0.006(6)$ & $0.002(5)$ \\
\hline$C(311)$ & $0.056(5)$ & $0.041(3)$ & $0.023(5)$ & $-0.001(4)$ & $0.004(5)$ & $0.001(5)$ \\
\hline$C(312)$ & $0.065(7)$ & $0.041(5)$ & $0.051(7)$ & $-0.009(5)$ & $0.012(6)$ & $0.000(6)$ \\
\hline$C(313)$ & $0.090(8)$ & $0.040(5)$ & $0.058(8)$ & $-0.011(6)$ & $0.015(8)$ & $0.004(6)$ \\
\hline$C(314)$ & $0.095(8)$ & $0.047(7)$ & $0.055(8)$ & $0.002(5)$ & $0.010(8)$ & $0.003(7)$ \\
\hline$C(315)$ & $0.088(8)$ & $0.046(5)$ & $0.060(8)$ & $0.019(6)$ & $0.028(8)$ & $0.010(7)$ \\
\hline$C(316)$ & $0.059(6)$ & $0.047(5)$ & $0.055(8)$ & $0.006(6)$ & $-0.001(7)$ & $-0.005(6)$ \\
\hline$C(321)$ & $0.054(5)$ & $0.035(6)$ & $0.039(5)$ & $-0.004(5)$ & $0.015(4)$ & $0.001(5)$ \\
\hline$C(322)$ & $0.059(6)$ & $0.057(8)$ & $0.036(6)$ & $0.005(6)$ & $-0.002(4)$ & $-0.004(6)$ \\
\hline$C(323)$ & $0.058(8)$ & $0.08(1)$ & $0.068(8)$ & $0.000(8)$ & $0.013(6)$ & $-0.012(7)$ \\
\hline$C(324)$ & $0.077(9)$ & $0.08(1)$ & $0.071(8)$ & $-0.003(7)$ & $0.037(7)$ & $-0.014(7)$ \\
\hline$C(325)$ & $0.104(10)$ & $0.078(10)$ & $0.043(8)$ & $0.019(9)$ & $0.034(7)$ & $0.018(7)$ \\
\hline$C(326)$ & $0.083(8)$ & $0.052(7)$ & $0.033(5)$ & $0.010(7)$ & $-0.004(5)$ & $-0.003(5)$ \\
\hline$C(411)$ & $0.056(7)$ & $0.047(6)$ & $0.028(4)$ & $0.001(5)$ & $0.016(5)$ & $0.001(4)$ \\
\hline$C(412)$ & $0.058(7)$ & $0.045(6)$ & $0.033(6)$ & $-0.006(5)$ & $0.002(5)$ & $0.005(4)$ \\
\hline$C(413)$ & $0.080(9)$ & $0.052(7)$ & $0.043(5)$ & $0.005(7)$ & $0.019(6)$ & $-0.002(5)$ \\
\hline$C(414)$ & $0.09(1)$ & $0.073(8)$ & $0.039(5)$ & $0.004(7)$ & $0.011(7)$ & $-0.013(6)$ \\
\hline$C(415)$ & $0.10(1)$ & $0.075(9)$ & $0.034(7)$ & $0.002(7)$ & $0.017(7)$ & $0.004(6)$ \\
\hline$C(416)$ & $0.070(9)$ & $0.071(9)$ & $0.031(5)$ & $0.006(7)$ & $0.005(6)$ & $0.015(5)$ \\
\hline$C(421)$ & $0.064(6)$ & $0.040(4)$ & $0.027(5)$ & $-0.010(4)$ & $0.001(5)$ & $0.001(5)$ \\
\hline$C(422)$ & $0.059(7)$ & $0.051(7)$ & $0.13(1)$ & $-0.006(7)$ & $0.021(9)$ & $0.010(9)$ \\
\hline$C(423)$ & $0.08(1)$ & $0.047(6)$ & $0.15(2)$ & $-0.010(6)$ & $0.04(1)$ & $0.00(1)$ \\
\hline$C(424)$ & $0.083(7)$ & $0.041(7)$ & $0.077(10)$ & $-0.015(6)$ & $0.001(8)$ & $0.010(7)$ \\
\hline$C(425)$ & $0.077(7)$ & $0.045(6)$ & $0.049(8)$ & $0.001(6)$ & $-0.023(6)$ & $0.001(6)$ \\
\hline$C(426)$ & $0.058(8)$ & $0.051(5)$ & $0.054(8)$ & $0.002(5)$ & $0.007(7)$ & $0.003(6)$ \\
\hline$C(511)$ & $0.052(3)$ & $0.033(5)$ & $0.039(5)$ & $0.002(5)$ & $0.006(4)$ & $-0.006(4)$ \\
\hline$C(512)$ & $0.058(5)$ & $0.049(7)$ & $0.038(5)$ & $-0.007(5)$ & $0.004(5)$ & $-0.001(6)$ \\
\hline$C(513)$ & $0.061(6)$ & $0.057(7)$ & $0.046(7)$ & $0.000(6)$ & $0.013(5)$ & $-0.011(5)$ \\
\hline$C(514)$ & $0.059(8)$ & $0.046(7)$ & $0.063(7)$ & $0.003(6)$ & $0.009(5)$ & $0.003(6)$ \\
\hline
\end{tabular}




\begin{tabular}{llllrrr}
$\mathrm{C}(515)$ & $0.061(6)$ & $0.056(8)$ & $0.053(7)$ & $0.010(7)$ & $-0.009(6)$ & $0.009(6)$ \\
$\mathrm{C}(516)$ & $0.062(6)$ & $0.054(7)$ & $0.042(7)$ & $0.002(6)$ & $-0.001(5)$ & $0.014(6)$ \\
$\mathrm{C}(611)$ & $0.059(3)$ & $0.042(6)$ & $0.037(5)$ & $0.007(6)$ & $-0.001(4)$ & $-0.001(5)$ \\
$\mathrm{C}(612)$ & $0.057(6)$ & $0.062(8)$ & $0.041(7)$ & $-0.001(7)$ & $-0.001(5)$ & $-0.009(6)$ \\
$\mathrm{C}(613)$ & $0.055(6)$ & $0.059(8)$ & $0.091(9)$ & $0.003(7)$ & $-0.005(7)$ & $0.010(8)$ \\
$\mathrm{C}(614)$ & $0.069(9)$ & $0.071(10)$ & $0.094(10)$ & $0.005(8)$ & $-0.030(7)$ & $0.012(9)$ \\
$\mathrm{C}(615)$ & $0.087(7)$ & $0.09(1)$ & $0.055(9)$ & $0.000(9)$ & $-0.032(6)$ & $0.015(8)$ \\
$\mathrm{C}(616)$ & $0.082(7)$ & $0.080(9)$ & $0.037(6)$ & $0.004(9)$ & $-0.012(6)$ & $0.004(7)$ \\
$\mathrm{C}(621)$ & $0.061(7)$ & $0.048(5)$ & $0.021(4)$ & $-0.013(5)$ & $0.004(4)$ & $-0.005(4)$ \\
$\mathrm{C}(622)$ & $0.075(8)$ & $0.045(7)$ & $0.028(5)$ & $0.002(6)$ & $0.012(5)$ & $0.007(4)$ \\
$\mathrm{C}(623)$ & $0.083(10)$ & $0.059(6)$ & $0.022(6)$ & $-0.007(7)$ & $0.018(5)$ & $0.001(5)$ \\
$\mathrm{C}(624)$ & $0.08(1)$ & $0.058(6)$ & $0.047(7)$ & $-0.006(8)$ & $0.023(7)$ & $-0.007(6)$ \\
$\mathrm{C}(625)$ & $0.081(9)$ & $0.047(7)$ & $0.051(7)$ & $0.012(7)$ & $0.014(6)$ & $0.004(5)$ \\
$\mathrm{C}(626)$ & $0.063(8)$ & $0.051(5)$ & $0.043(7)$ & $-0.004(6)$ & $0.008(6)$ & $0.007(5)$ \\
$\mathrm{C}(701)$ & $0.12(1)$ & $0.066(10)$ & $0.040(9)$ & $-0.016(9)$ & $-0.018(6)$ & $0.012(6)$ \\
$\mathrm{C}(702)$ & $0.16(2)$ & $0.08(1)$ & $0.051(9)$ & $0.02(1)$ & $0.013(9)$ & $0.000(8)$ \\
C(703) & $0.10(1)$ & $0.10(1)$ & $0.09(1)$ & $-0.021(8)$ & $0.02(1)$ & $-0.03(1)$ \\
C(704) & $0.10(2)$ & $0.12(2)$ & $0.22(3)$ & $0.01(1)$ & $0.01(2)$ & $-0.03(2)$ \\
\hline
\end{tabular}

a Estmated standard deviations are given in parentheses. $b$ Parameters have the form exp($\left.2 \pi^{2}\left(U_{11} h^{2} a * 2+\cdots+2 U_{12} h k a^{*} b^{*} \cos \gamma+\cdots\right)\right)$.

Table S5-4. Bond Lengths for Non-hydrogen Atoms of $6 \mathrm{c} \cdot 2 \mathrm{CH}_{3} \mathrm{CN}^{\mathrm{a}}$

\begin{tabular}{|c|c|c|c|c|c|}
\hline atom & atom & distance & atom & atom & distance \\
\hline $\operatorname{Pt}(1)$ & $\operatorname{Pt}(2)$ & $2.7696(7)$ & $\mathrm{P}(5)$ & $C(9)$ & $1.85(1)$ \\
\hline $\operatorname{Pt}(1)$ & $P(1)$ & $2.278(4)$ & $P(5)$ & $C(10)$ & $1.79(1)$ \\
\hline $\operatorname{Pt}(1)$ & $\mathrm{P}(4)$ & $2.277(3)$ & $P(5)$ & $C(511)$ & $1.89(1)$ \\
\hline $\operatorname{Pt}(1)$ & $C(1)$ & $2.01(1)$ & $P(6)$ & $C(10)$ & $1.84(1)$ \\
\hline $\operatorname{Pt}(2)$ & $\operatorname{Pt}(3)$ & $2.7506(7)$ & $P(6)$ & $C(611)$ & $1.88(1)$ \\
\hline $\operatorname{Pt}(2)$ & $P(2)$ & $2.271(3)$ & $P(6)$ & $C(621)$ & $1.85(1)$ \\
\hline $\operatorname{Pt}(2)$ & $P(5)$ & $2.268(3)$ & $\mathrm{N}(1)$ & $C(1)$ & $1.18(2)$ \\
\hline $\operatorname{Pt}(3)$ & $P(3)$ & $2.296(3)$ & $\mathrm{N}(1)$ & $C(11)$ & $1.45(2)$ \\
\hline $\operatorname{Pt}(3)$ & $P(6)$ & $2.299(3)$ & $\mathrm{N}(2)$ & $C(2)$ & $1.18(2)$ \\
\hline $\operatorname{Pt}(3)$ & $C(2)$ & $2.00(1)$ & $\mathrm{N}(2)$ & $C(21)$ & $1.41(2)$ \\
\hline $\operatorname{Pt}(4)$ & $C(3)$ & $2.04(2)$ & $\mathrm{N}(3)$ & $C(3)$ & $1.15(2)$ \\
\hline $\operatorname{Pt}(4)$ & $C(4)$ & $2.09(2)$ & $\mathrm{N}(4)$ & $C(4)$ & $1.13(2)$ \\
\hline $\operatorname{Pt}(4)$ & $C(5)$ & $2.03(1)$ & $\mathrm{N}(5)$ & $C(5)$ & $1.12(2)$ \\
\hline $\operatorname{Pt}(4)$ & $C(6)$ & $2.05(2)$ & $\mathrm{N}(6)$ & $C(6)$ & $1.20(2)$ \\
\hline $\mathrm{P}(1)$ & $C(7)$ & $1.83(1)$ & $\mathrm{N}(701)$ & $C(701)$ & $1.16(2)$ \\
\hline$P(1)$ & $C(111)$ & $1.85(1)$ & $\mathrm{N}(702)$ & $C(703)$ & $1.15(3)$ \\
\hline$P(1)$ & $C(121)$ & $1.88(1)$ & $C(11)$ & $C(12)$ & $1.41(2)$ \\
\hline $\mathrm{P}(2)$ & $C(7)$ & 1.87(1) & $C(11)$ & $C(16)$ & $1.42(2)$ \\
\hline $\mathrm{P}(2)$ & $C(8)$ & $1.85(1)$ & $C(12)$ & $C(13)$ & $1.44(2)$ \\
\hline $\mathrm{P}(2)$ & $C(211)$ & $1.81(1)$ & $C(12)$ & $C(17)$ & $1.48(2)$ \\
\hline $\mathrm{P}(3)$ & $C(8)$ & $1.86(1)$ & $C(13)$ & $C(14)$ & $1.36(2)$ \\
\hline$P(3)$ & $C(311)$ & $1.84(1)$ & $C(14)$ & $C(15)$ & $1.45(2)$ \\
\hline$P(3)$ & $C(321)$ & 1.87(1) & $C(15)$ & $C(16)$ & $1.41(2)$ \\
\hline $\mathrm{P}(4)$ & $C(9)$ & $1.88(1)$ & $C(16)$ & $C(18)$ & $1.53(2)$ \\
\hline $\mathrm{P}(4)$ & $C(411)$ & $1.85(1)$ & $C(21)$ & $C(22)$ & $1.40(2)$ \\
\hline $\mathrm{P}(4)$ & $C(421)$ & $1.83(1)$ & $C(21)$ & $C(26)$ & $1.43(2)$ \\
\hline$C(22)$ & $C(23)$ & $1.42(2)$ & $C(312)$ & $C(313)$ & $1.39(2)$ \\
\hline$C(22)$ & $C(27)$ & $1.57(2)$ & $C(313)$ & $C(314)$ & $1.41(2)$ \\
\hline$C(23)$ & $C(24)$ & $1.41(2)$ & $C(314)$ & $C(315)$ & $1.41(2)$ \\
\hline$C(24)$ & $C(25)$ & $1.40(2)$ & $C(315)$ & $C(316)$ & $1.38(2)$ \\
\hline$C(25)$ & $C(26)$ & $1.38(2)$ & $C(321)$ & $C(322)$ & $1.41(2)$ \\
\hline$C(26)$ & $C(28)$ & $1.50(2)$ & $C(321)$ & $C(326)$ & $1.41(2)$ \\
\hline
\end{tabular}




\begin{tabular}{|c|c|c|c|c|c|}
\hline$C(111)$ & $C(112)$ & $1.43(2)$ & $C(322)$ & $C(323)$ & $1.43(2)$ \\
\hline $\mathrm{C}(111)$ & $C(116)$ & $1.40(2)$ & $C(323)$ & $C(324)$ & $1.38(2)$ \\
\hline$C(112)$ & $C(113)$ & $1.44(2)$ & $C(324)$ & $C(325)$ & $1.44(2)$ \\
\hline$C(113)$ & $C(114)$ & $1.36(2)$ & $C(325)$ & $C(326)$ & $1.40(2)$ \\
\hline$C(114)$ & $C(115)$ & $1.38(2)$ & $C(411)$ & $C(412)$ & $1.41(2)$ \\
\hline$C(115)$ & $C(116)$ & $1.41(2)$ & $C(411)$ & $C(416)$ & $1.39(2)$ \\
\hline$C(121)$ & $C(122)$ & $1.42(2)$ & $C(412)$ & $C(413)$ & $1.40(2)$ \\
\hline$C(121)$ & $C(126)$ & $1.40(2)$ & $C(413)$ & $C(414)$ & $1.36(2)$ \\
\hline$C(122)$ & $C(123)$ & $1.39(2)$ & $C(414)$ & $C(415)$ & $1.37(2)$ \\
\hline$C(123)$ & $C(124)$ & $1.38(2)$ & $C(415)$ & $C(416)$ & $1.39(2)$ \\
\hline$C(124)$ & $C(125)$ & $1.43(2)$ & $C(421)$ & $C(422)$ & $1.43(2)$ \\
\hline$C(125)$ & $C(126)$ & $1.43(2)$ & $C(421)$ & $C(426)$ & $1.43(2)$ \\
\hline$C(211)$ & $C(212)$ & $1.45(2)$ & $C(422)$ & $C(423)$ & $1.36(2)$ \\
\hline$C(211)$ & $C(216)$ & $1.43(2)$ & $C(423)$ & $C(424)$ & $1.42(2)$ \\
\hline$C(212)$ & $C(213)$ & $1.38(2)$ & $C(424)$ & $C(425)$ & $1.42(2)$ \\
\hline$C(213)$ & $C(214)$ & $1.41(2)$ & $C(425)$ & $C(426)$ & $1.41(2)$ \\
\hline$C(214)$ & $C(215)$ & $1.37(2)$ & $C(511)$ & $C(512)$ & $1.38(2)$ \\
\hline$C(215)$ & $C(216)$ & $1.40(2)$ & $C(511)$ & $C(516)$ & $1.44(2)$ \\
\hline$C(311)$ & $C(312)$ & $1.40(2)$ & $C(512)$ & $C(513)$ & $1.43(2)$ \\
\hline$C(311)$ & $C(316)$ & $1.43(2)$ & $C(513)$ & $C(514)$ & $1.39(2)$ \\
\hline$C(514)$ & $C(515)$ & $1.39(2)$ & & & \\
\hline$C(515)$ & $C(516)$ & $1.44(2)$ & & & \\
\hline$C(611)$ & $C(612)$ & $1.44(2)$ & & & \\
\hline$C(611)$ & $C(616)$ & $1.39(2)$ & & & \\
\hline$C(612)$ & $C(613)$ & $1.45(2)$ & & & \\
\hline$C(613)$ & $C(614)$ & $1.36(2)$ & & & \\
\hline$C(614)$ & $C(615)$ & $1.42(2)$ & & & \\
\hline$C(615)$ & $C(616)$ & $1.43(2)$ & & & \\
\hline$C(621)$ & $C(622)$ & $1.39(2)$ & & & \\
\hline$C(621)$ & $C(626)$ & $1.38(2)$ & & & \\
\hline$C(622)$ & $C(623)$ & $1.39(2)$ & & & \\
\hline$C(623)$ & $C(624)$ & $1.37(2)$ & & & \\
\hline$C(624)$ & $C(625)$ & $1.36(2)$ & & & \\
\hline$C(625)$ & $C(626)$ & $1.40(2)$ & & & \\
\hline$C(701)$ & $C(702)$ & $1.42(3)$ & & & \\
\hline$C(703)$ & $C(704)$ & $1.45(3)$ & & & \\
\hline
\end{tabular}

a Estimated standard deviations are given in parentheses.

Table S5-5. Bond Angles for Non-hydrogen Atoms of $6 \mathbf{c} \cdot 2 \mathrm{CH}_{3} \mathrm{CN}^{\mathrm{a}}$

\begin{tabular}{lccccccc}
\hline atom & atom & atom & angle & atom & atom & atom & angle \\
\hline $\operatorname{Pt}(2)$ & $\operatorname{Pt}(1)$ & $\mathrm{P}(1)$ & $83.78(9)$ & $\mathrm{Pt}(1)$ & $\mathrm{P}(1)$ & $\mathrm{C}(121)$ & $116.4(4)$ \\
$\operatorname{Pt}(2)$ & $\operatorname{Pt}(1)$ & $\mathrm{P}(4)$ & $84.42(9)$ & $\mathrm{C}(7)$ & $\mathrm{P}(1)$ & $\mathrm{C}(111)$ & $103.8(6)$ \\
$\operatorname{Pt}(2)$ & $\operatorname{Pt}(1)$ & $\mathrm{C}(1)$ & $173.3(4)$ & $\mathrm{C}(7)$ & $\mathrm{P}(1)$ & $\mathrm{C}(121)$ & $105.4(6)$ \\
$\mathrm{P}(1)$ & $\operatorname{Pt}(1)$ & $\mathrm{P}(4)$ & $168.0(1)$ & $\mathrm{C}(111)$ & $\mathrm{P}(1)$ & $\mathrm{C}(121)$ & $105.4(6)$ \\
$\mathrm{P}(1)$ & $\operatorname{Pt}(1)$ & $\mathrm{C}(1)$ & $95.4(4)$ & $\mathrm{Pt}(2)$ & $\mathrm{P}(2)$ & $\mathrm{C}(7)$ & $117.2(4)$ \\
$\mathrm{P}(4)$ & $\operatorname{Pt}(1)$ & $\mathrm{C}(1)$ & $96.6(4)$ & $\mathrm{Pt}(2)$ & $\mathrm{P}(2)$ & $\mathrm{C}(8)$ & $109.3(4)$ \\
$\operatorname{Pt}(1)$ & $\operatorname{Pt}(2)$ & $\mathrm{Pt}(3)$ & $175.99(2)$ & $\mathrm{Pt}(2)$ & $\mathrm{P}(2)$ & $\mathrm{C}(211)$ & $117.0(4)$ \\
$\operatorname{Pt}(1)$ & $\operatorname{Pt}(2)$ & $\mathrm{P}(2)$ & $89.15(9)$ & $\mathrm{C}(7)$ & $\mathrm{P}(2)$ & $\mathrm{C}(8)$ & $102.9(6)$ \\
$\operatorname{Pt}(1)$ & $\operatorname{Pt}(2)$ & $\mathrm{P}(5)$ & $95.24(9)$ & $\mathrm{C}(7)$ & $\mathrm{P}(2)$ & $\mathrm{C}(211)$ & $102.7(6)$ \\
$\operatorname{Pt}(3)$ & $\operatorname{Pt}(2)$ & $\mathrm{P}(2)$ & $88.83(9)$ & $\mathrm{C}(8)$ & $\mathrm{P}(2)$ & $\mathrm{C}(211)$ & $106.5(6)$ \\
$\operatorname{Pt}(3)$ & $\operatorname{Pt}(2)$ & $\mathrm{P}(5)$ & $86.47(8)$ & $\mathrm{Pt}(3)$ & $\mathrm{P}(3)$ & $\mathrm{C}(8)$ & $116.0(4)$ \\
$\mathrm{P}(2)$ & $\operatorname{Pt}(2)$ & $\mathrm{P}(5)$ & $173.1(1)$ & $\mathrm{Pt}(3)$ & $\mathrm{P}(3)$ & $\mathrm{C}(311)$ & $112.6(4)$ \\
$\operatorname{Pt}(2)$ & $\operatorname{Pt}(3)$ & $\mathrm{P}(3)$ & $89.13(9)$ & $\mathrm{Pt}(3)$ & $\mathrm{P}(3)$ & $\mathrm{C}(321)$ & $110.5(4)$ \\
$\operatorname{Pt}(2)$ & $\operatorname{Pt}(3)$ & $\mathrm{P}(6)$ & $90.22(9)$ & $\mathrm{C}(8)$ & $\mathrm{P}(3)$ & $\mathrm{C}(311)$ & $103.0(6)$ \\
$\operatorname{Pt}(2)$ & $\operatorname{Pt}(3)$ & $\mathrm{C}(2)$ & $176.1(3)$ & $\mathrm{C}(8)$ & $\mathrm{P}(3)$ & $\mathrm{C}(321)$ & $109.4(6)$
\end{tabular}




\begin{tabular}{|c|c|c|c|c|c|c|c|}
\hline $\mathrm{P}(3)$ & $\operatorname{Pt}(3)$ & $P(6)$ & 179.3(1) & $C(311)$ & $\mathrm{P}(3)$ & $C(321)$ & $104.6(6)$ \\
\hline $\mathrm{P}(3)$ & $\operatorname{Pt}(3)$ & $C(2)$ & $87.5(3)$ & $\operatorname{Pt}(1)$ & $\mathrm{P}(4)$ & $C(9)$ & $110.1(4)$ \\
\hline $\mathrm{P}(6)$ & $\operatorname{Pt}(3)$ & $C(2)$ & 93.2(3) & $\operatorname{Pt}(1)$ & $\mathrm{P}(4)$ & $C(411)$ & $113.8(4)$ \\
\hline$C(3)$ & $\operatorname{Pt}(4)$ & $C(4)$ & $88.9(7)$ & $\operatorname{Pt}(1)$ & $\mathrm{P}(4)$ & $C(421)$ & $116.6(4)$ \\
\hline$C(3)$ & $\operatorname{Pt}(4)$ & $C(5)$ & 178.0(7) & $C(9)$ & $\mathrm{P}(4)$ & $C(411)$ & 109.1(6) \\
\hline$C(3)$ & $\operatorname{Pt}(4)$ & $C(6)$ & 93.1(7) & $C(9)$ & $\mathrm{P}(4)$ & $C(421)$ & 104.1(6) \\
\hline$C(4)$ & $\operatorname{Pt}(4)$ & $C(5)$ & $89.2(6)$ & $C(411)$ & $\mathrm{P}(4)$ & $C(421)$ & $102.5(6)$ \\
\hline$C(4)$ & $\operatorname{Pt}(4)$ & $C(6)$ & $176.9(6)$ & $\operatorname{Pt}(2)$ & $P(5)$ & $C(9)$ & $112.8(4)$ \\
\hline$C(5)$ & $\operatorname{Pt}(4)$ & $C(6)$ & $88.8(6)$ & $\operatorname{Pt}(2)$ & $P(5)$ & $C(10)$ & $107.7(4)$ \\
\hline $\operatorname{Pt}(1)$ & $\mathrm{P}(1)$ & $C(7)$ & 108.1(4) & $\operatorname{Pt}(2)$ & $P(5)$ & $C(511)$ & $121.6(4)$ \\
\hline $\operatorname{Pt}(1)$ & $\mathrm{P}(1)$ & $C(111)$ & $116.5(4)$ & $C(9)$ & $\mathrm{P}(5)$ & $C(10)$ & 102.4(6) \\
\hline$C(9)$ & $P(5)$ & $C(511)$ & 105.6(6) & $C(12)$ & $C(13)$ & $C(14)$ & $122(1)$ \\
\hline$C(10)$ & $P(5)$ & $C(511)$ & $104.8(6)$ & $C(13)$ & $C(14)$ & $C(15)$ & $118(1)$ \\
\hline $\operatorname{Pt}(3)$ & $P(6)$ & $C(10)$ & $115.0(4)$ & $C(14)$ & $C(15)$ & $C(16)$ & 124(1) \\
\hline $\operatorname{Pt}(3)$ & $P(6)$ & $C(611)$ & $112.6(4)$ & $C(11)$ & $C(16)$ & $C(15)$ & 114(1) \\
\hline $\operatorname{Pt}(3)$ & $P(6)$ & $C(621)$ & $112.8(4)$ & $C(11)$ & $C(16)$ & C(18) & 122(1) \\
\hline$C(10)$ & $P(6)$ & $C(611)$ & $104.8(6)$ & $C(15)$ & $C(16)$ & $C(18)$ & 124(1) \\
\hline$C(10)$ & $P(6)$ & $C(621)$ & 102.9(5) & $\mathrm{N}(2)$ & $C(21)$ & $C(22)$ & 114(1) \\
\hline$C(611)$ & $P(6)$ & $C(621)$ & $107.9(6)$ & $\mathrm{N}(2)$ & $C(21)$ & $C(26)$ & 121(1) \\
\hline$C(1)$ & $\mathrm{N}(1)$ & $C(11)$ & 178(1) & $C(22)$ & $C(21)$ & $C(26)$ & $125(1)$ \\
\hline$C(2)$ & $\mathrm{N}(2)$ & $C(21)$ & 177(1) & $C(21)$ & $C(22)$ & $C(23)$ & 114(1) \\
\hline $\operatorname{Pt}(1)$ & $C(1)$ & $\mathrm{N}(1)$ & 175(1) & $C(21)$ & $C(22)$ & $C(27)$ & $123(1)$ \\
\hline $\operatorname{Pt}(3)$ & $C(2)$ & $\mathrm{N}(2)$ & 174(1) & $C(23)$ & $C(22)$ & $C(27)$ & $123(1)$ \\
\hline $\operatorname{Pt}(4)$ & $C(3)$ & $\mathrm{N}(3)$ & $175(2)$ & $C(22)$ & $C(23)$ & $C(24)$ & 122(1) \\
\hline $\operatorname{Pt}(4)$ & $C(4)$ & $\mathrm{N}(4)$ & $174(2)$ & $C(23)$ & $C(24)$ & $C(25)$ & 121(1) \\
\hline $\operatorname{Pt}(4)$ & $C(5)$ & $N(5)$ & 175(1) & $C(24)$ & $C(25)$ & $C(26)$ & 119(1) \\
\hline $\operatorname{Pt}(4)$ & $C(6)$ & $\mathrm{N}(6)$ & 178(1) & $C(21)$ & $C(26)$ & $C(25)$ & 119(1) \\
\hline $\mathrm{P}(1)$ & $C(7)$ & $\mathrm{P}(2)$ & 102.8(6) & $C(21)$ & $C(26)$ & $C(28)$ & $122(1)$ \\
\hline $\mathrm{P}(2)$ & $C(8)$ & $\mathrm{P}(3)$ & $110.2(6)$ & $C(25)$ & $C(26)$ & $C(28)$ & 119(1) \\
\hline $\mathrm{P}(4)$ & $C(9)$ & $P(5)$ & 107.3(6) & $\mathrm{P}(1)$ & $C(111)$ & $C(112)$ & 124(1) \\
\hline $\mathrm{P}(5)$ & $C(10)$ & $P(6)$ & 106.6(6) & $\mathrm{P}(1)$ & $C(111)$ & $C(116)$ & 117(1) \\
\hline $\mathrm{N}(1)$ & $C(11)$ & $C(12)$ & 121(1) & $C(112)$ & $C(111)$ & $C(116)$ & $119(1)$ \\
\hline $\mathrm{N}(1)$ & $C(11)$ & $C(16)$ & $115(1)$ & $C(111)$ & $C(112)$ & $C(113)$ & 119(1) \\
\hline$C(12)$ & $C(11)$ & $C(16)$ & $124(1)$ & $C(112)$ & $C(113)$ & $C(114)$ & 120(1) \\
\hline$C(11)$ & $C(12)$ & $C(13)$ & 118(1) & $C(113)$ & $C(114)$ & $C(115)$ & 122(1) \\
\hline$C(11)$ & $C(12)$ & $C(17)$ & 121(1) & $C(114)$ & $C(115)$ & $C(116)$ & 121(1) \\
\hline$C(13)$ & $C(12)$ & $C(17)$ & $121(1)$ & $C(111)$ & $C(116)$ & $C(115)$ & $120(1)$ \\
\hline $\mathrm{P}(1)$ & $C(121)$ & $C(122)$ & $124(1)$ & $C(322)$ & $C(321)$ & $C(326)$ & 119(1) \\
\hline $\mathrm{P}(1)$ & $C(121)$ & $C(126)$ & 118(1) & $C(321)$ & $C(322)$ & $C(323)$ & $122(1)$ \\
\hline$C(122)$ & $C(121)$ & $C(126)$ & 118(1) & $C(322)$ & $C(323)$ & $C(324)$ & $120(2)$ \\
\hline$C(121)$ & $C(122)$ & $C(123)$ & 123(1) & $C(323)$ & $C(324)$ & $C(325)$ & 117(1) \\
\hline$C(122)$ & $C(123)$ & $C(124)$ & 119(1) & $C(324)$ & $C(325)$ & $C(326)$ & $124(1)$ \\
\hline$C(123)$ & $C(124)$ & $C(125)$ & $120(1)$ & $C(321)$ & $C(326)$ & $C(325)$ & $117(1)$ \\
\hline$C(124)$ & $C(125)$ & $C(126)$ & 121(1) & $\mathrm{P}(4)$ & $C(411)$ & $C(412)$ & $119.4(9)$ \\
\hline$C(121)$ & $C(126)$ & $C(125)$ & 119(1) & $\mathrm{P}(4)$ & $C(411)$ & $C(416)$ & $120(1)$ \\
\hline $\mathrm{P}(2)$ & $C(211)$ & $C(212)$ & 119(1) & $C(412)$ & $C(411)$ & $C(416)$ & $120(1)$ \\
\hline $\mathrm{P}(2)$ & $C(211)$ & $C(216)$ & 123(1) & $C(411)$ & $C(412)$ & $C(413)$ & $119(1)$ \\
\hline$C(212)$ & $C(211)$ & $C(216)$ & 118(1) & $C(412)$ & $C(413)$ & $C(414)$ & 119(1) \\
\hline$C(211)$ & $C(212)$ & $C(213)$ & 118(1) & $C(413)$ & $C(414)$ & $C(415)$ & $122(1)$ \\
\hline$C(212)$ & $C(213)$ & $C(214)$ & 122(1) & $C(414)$ & $C(415)$ & $C(416)$ & $120(1)$ \\
\hline$C(213)$ & $C(214)$ & $C(215)$ & 121(1) & $C(411)$ & $C(416)$ & $C(415)$ & 119(1) \\
\hline$C(214)$ & $C(215)$ & $C(216)$ & $119(1)$ & $\mathrm{P}(4)$ & $C(421)$ & $C(422)$ & 121(1) \\
\hline$C(211)$ & $C(216)$ & $C(215)$ & 122(1) & $\mathrm{P}(4)$ & $C(421)$ & $C(426)$ & $120(1)$ \\
\hline $\mathrm{P}(3)$ & $C(311)$ & $C(312)$ & 121(1) & $C(422)$ & $C(421)$ & $C(426)$ & 119(1) \\
\hline $\mathrm{P}(3)$ & $C(311)$ & $C(316)$ & 118.6(9) & $C(421)$ & $C(422)$ & $C(423)$ & $123(2)$ \\
\hline$C(312)$ & $C(311)$ & $C(316)$ & 121(1) & $C(422)$ & $C(423)$ & $C(424)$ & $118(2)$ \\
\hline
\end{tabular}




\begin{tabular}{|c|c|c|c|c|c|c|c|}
\hline$C(311)$ & $C(312)$ & $C(313)$ & $120(1)$ & $C(423)$ & $C(424)$ & $C(425)$ & $120(1)$ \\
\hline$C(312)$ & $C(313)$ & $C(314)$ & 119(1) & $C(424)$ & $C(425)$ & $C(426)$ & $122(1)$ \\
\hline$C(313)$ & $C(314)$ & $C(315)$ & 121(1) & $C(421)$ & $C(426)$ & $C(425)$ & $117(1)$ \\
\hline$C(314)$ & $C(315)$ & $C(316)$ & $119(1)$ & $P(5)$ & $C(511)$ & $C(512)$ & $122(1)$ \\
\hline$C(311)$ & $C(316)$ & $C(315)$ & $120(1)$ & $P(5)$ & $C(511)$ & $C(516)$ & $122(1)$ \\
\hline $\mathrm{P}(3)$ & $C(321)$ & $C(322)$ & $122(1)$ & $C(512)$ & $C(511)$ & $C(516)$ & $116(1)$ \\
\hline$P(3)$ & $C(321)$ & $C(326)$ & $119(1)$ & $C(511)$ & $C(512)$ & $C(513)$ & $123(1)$ \\
\hline$C(512)$ & $C(513)$ & $C(514)$ & $121(1)$ & & & & \\
\hline$C(513)$ & $C(514)$ & $C(515)$ & 119(1) & & & & \\
\hline$C(514)$ & $C(515)$ & $C(516)$ & 121(1) & & & & \\
\hline$C(511)$ & $C(516)$ & $C(515)$ & 121(1) & & & & \\
\hline$P(6)$ & $C(611)$ & $C(612)$ & $123(1)$ & & & & \\
\hline$P(6)$ & $C(611)$ & $C(616)$ & $120(1)$ & & & & \\
\hline$C(612)$ & $C(611)$ & $C(616)$ & $117(1)$ & & & & \\
\hline$C(611)$ & $C(612)$ & $C(613)$ & 121(1) & & & & \\
\hline$C(612)$ & $C(613)$ & $C(614)$ & $121(2)$ & & & & \\
\hline$C(613)$ & $C(614)$ & $C(615)$ & $118(2)$ & & & & \\
\hline$C(614)$ & $C(615)$ & $C(616)$ & $122(1)$ & & & & \\
\hline$C(611)$ & $C(616)$ & $C(615)$ & $121(2)$ & & & & \\
\hline$P(6)$ & $C(621)$ & $C(622)$ & 121(1) & & & & \\
\hline$P(6)$ & $C(621)$ & $C(626)$ & $119.4(9)$ & & & & \\
\hline$C(622)$ & $C(621)$ & $C(626)$ & $119(1)$ & & & & \\
\hline$C(621)$ & $C(622)$ & $C(623)$ & $120(1)$ & & & & \\
\hline$C(622)$ & $C(623)$ & $C(624)$ & 121(1) & & & & \\
\hline$C(623)$ & $C(624)$ & $C(625)$ & $119(1)$ & & & & \\
\hline$C(624)$ & $C(625)$ & $C(626)$ & $121(1)$ & & & & \\
\hline$C(621)$ & $C(626)$ & $C(625)$ & $120(1)$ & & & & \\
\hline $\mathrm{N}(701)$ & $C(701)$ & $C(702)$ & $179(2)$ & & & & \\
\hline $\mathrm{N}(702)$ & $C(703)$ & $C(704)$ & $179(2)$ & & & & \\
\hline
\end{tabular}

a Estimated standard deviations are given in parentheses. 
Table S6-1. Crystallographic and Experimental Data for $7 \mathbf{a} \cdot 3 \mathrm{CH}_{2} \mathrm{Cl}_{2}$

\begin{tabular}{|c|c|}
\hline formula & $\mathrm{C}_{79} \mathrm{H}_{76} \mathrm{~N}_{2} \mathrm{P}_{8} \mathrm{~F}_{12} \mathrm{Pt}_{3} \mathrm{Cl}_{6}$ \\
\hline formula weight & 2327.24 \\
\hline color & reddish orange \\
\hline size, $\mathrm{mm}$ & $0.35 \times 0.20 \times 0.20$ \\
\hline cryst. system & monoclinic \\
\hline space group & $P 21 / \mathrm{C}$ (No. 14 ) \\
\hline no. refs used for cell determination & $14376\left(6<2 \theta<55^{\circ}\right)$ \\
\hline lattice const. & $\begin{array}{l}a=23.511(3) \AA \\
b=17.137(2) \AA \\
c=25.919(4) \AA \\
\beta=111.174(6){ }^{\circ} \\
V=9738(2) \AA^{3}\end{array}$ \\
\hline Z & 4 \\
\hline$D_{\text {calc }}, \mathrm{g} \mathrm{cm}^{-3}$ & 1.587 \\
\hline$\mu(\operatorname{Mo} K \alpha), \mathrm{cm}^{-1}$ & 46.39 (trans. $0.39 \sim 1.00$ ) \\
\hline$F(000)$ & 4512 \\
\hline \multicolumn{2}{|c|}{$\begin{array}{r}\text { An absorption correction was applied (multi-scan spherical method). } \\
\text { Rigaku Mercury CCD }(50 \mathrm{kV}, 190 \mathrm{~mA})\end{array}$} \\
\hline diffractometer & \\
\hline radiation & $\operatorname{Mo} K \alpha(\lambda=0.71069 \AA)$ \\
\hline monochromator & graphite \\
\hline & -120 \\
\hline $2 \theta$ range, ${ }^{\circ}$ & $6<2 \theta<50$ \\
\hline$h, k$, l range & $+\mathrm{h},+\mathrm{k}, \pm \mathrm{l}$ \\
\hline detector aperture, $\mathrm{mm}$ & $70 \times 70$ \\
\hline data images & 1440 \\
\hline$\omega$ oscillation range $(\chi=45, \phi=90), \circ$ & -70 to 110 \\
\hline exposure rate, ${ }^{\circ} \mathrm{sec}^{-1}$ & 80 \\
\hline detector swing angle, ${ }^{\circ}$ & 20 \\
\hline detector position, $\mathrm{mm}$ & 60 \\
\hline solution & Patterson Methods (DIRDIF94 Patty) \\
\hline \multirow[t]{2}{*}{ refinement } & full-matrix least-squares (teXsan) \\
\hline & $\begin{array}{l}\text { The } \mathrm{Pt}, \mathrm{Cl}, \mathrm{P} \text {, and } \mathrm{F} \text { atoms were refined with } \\
\text { anisotropic temperature factors, and other non- } \\
\text { hydrogen atoms with isotropic ones. }\end{array}$ \\
\hline hydrogen atoms & $\begin{array}{l}\text { All } \mathrm{H} \text { atom except } \mathrm{CH}_{2} \mathrm{Cl}_{2} \text { were calcd with } \mathrm{C}-\mathrm{H}= \\
0.95 \text {. } \mathrm{A} \text {, and were not refined or refined as rigid } \\
\text { phenyl groups. }\end{array}$ \\
\hline function minimized & $\Sigma w\left(\left|F_{0}\right|-|F c|\right)^{2}$ \\
\hline weighting scheme & $1 / \sigma^{2}(F o)$ \\
\hline anomalous dispersion & all non-hydrogen atoms \\
\hline no. unique reflections & 19101 \\
\hline$R_{\text {int }}$ & 0.046 \\
\hline no. obsd. unique reflections & $8590(I>2 \sigma(I))$ \\
\hline$p$-factor & 0.03 \\
\hline no. variables & 619 \\
\hline data / param ratio & 13.88 \\
\hline$R^{\mathrm{a}}$ & 0.084 \\
\hline$R_{\mathrm{W}} \mathrm{b}$ & 0.091 \\
\hline GOF c & 2.22 \\
\hline$\rho_{\max }, \mathrm{e}^{-3}$ & $2.54,-1.01$ \\
\hline a $R=\Sigma|| F_{\mathrm{O}}|-| F_{\mathrm{C}}|| / \Sigma\left|F_{\mathrm{O}}\right|$ & \\
\hline $\mathrm{b}_{R_{\mathrm{W}}}=\left[\Sigma w\left(\left|F_{\mathrm{O}}\right|-\left|F_{\mathrm{C}}\right|\right)^{2} / \Sigma w\left|F_{\mathrm{O}}\right|^{2}\right]$ & $\left.\left(F_{\mathrm{O}}\right)\right)$ \\
\hline
\end{tabular}


Table S6-2. Final Positional Parameters and $\mathrm{B}_{\mathrm{eq}}$ for $7 \mathbf{a} \cdot 3 \mathrm{CH}_{2} \mathrm{Cl}_{2} \mathrm{a}, \mathrm{b}, \mathrm{c}$

\begin{tabular}{|c|c|c|c|c|}
\hline atom & $x$ & $y$ & $z$ & $B$ eq \\
\hline$\overline{P t}(1)$ & $0.23259(4)$ & $0.04722(3)$ & $0.44871(3)$ & $2.90(1)$ \\
\hline $\operatorname{Pt}(2)$ & $0.23456(4)$ & $0.20478(3)$ & $0.45297(3)$ & $2.28(1)$ \\
\hline $\operatorname{Pt}(3)$ & $0.23664(4)$ & $0.36206(3)$ & $0.45633(3)$ & $3.03(2)$ \\
\hline $\mathrm{Cl}(1)$ & $0.3743(5)$ & $-0.4171(6)$ & $0.6887(4)$ & $12.9(3)$ \\
\hline $\mathrm{Cl}(2)$ & $0.4652(5)$ & $-0.3002(7)$ & $0.7018(4)$ & $14.3(4)$ \\
\hline $\mathrm{Cl}(3)$ & $0.4681(7)$ & $-0.0464(8)$ & $0.7385(6)$ & $18.9(5)$ \\
\hline $\mathrm{Cl}(4)$ & $0.4168(9)$ & $0.024(1)$ & $0.8157(6)$ & $24.3(7)$ \\
\hline $\mathrm{Cl}(5)$ & $0.2864(7)$ & $-0.246(1)$ & $0.6928(6)$ & $21.3(6)$ \\
\hline $\mathrm{Cl}(6)$ & $0.2777(6)$ & $-0.071(1)$ & $0.6854(7)$ & $20.9(6)$ \\
\hline $\mathrm{P}(11)^{\mathrm{e}}$ & $0.3220(4)$ & $0.0538(5)$ & $0.5177(3)$ & $1.7(2)$ \\
\hline$P(12)^{e}$ & $0.3368(4)$ & $0.2051(5)$ & $0.4724(3)$ & $1.3(1)$ \\
\hline $\mathrm{P}(13)^{\mathrm{e}}$ & $0.3304(4)$ & $0.3552(5)$ & $0.5277(3)$ & $2.0(2)$ \\
\hline $\mathrm{P}(21)^{\mathrm{e}}$ & $0.3407(5)$ & $0.0505(6)$ & $0.4729(5)$ & $4.0(3)$ \\
\hline$P(22)^{e}$ & $0.3266(4)$ & $0.1986(6)$ & $0.5255(5)$ & $3.4(2)$ \\
\hline$P(23)^{e}$ & $0.3380(6)$ & $0.3499(6)$ & $0.4804(5)$ & $4.8(3)$ \\
\hline $\mathrm{P}(31)^{\mathrm{e}}$ & $0.1367(4)$ & $0.0610(5)$ & $0.3806(4)$ & $2.1(2)$ \\
\hline$P(32)^{e}$ & $0.1331(4)$ & $0.2116(4)$ & $0.4330(3)$ & $1.5(2)$ \\
\hline$P(33)^{e}$ & $0.1459(4)$ & $0.3649(5)$ & $0.3835(4)$ & $2.2(2)$ \\
\hline $\mathrm{P}(41)^{\mathrm{e}}$ & $0.1332(5)$ & $0.0497(6)$ & $0.4309(5)$ & $4.0(3)$ \\
\hline$P(42)^{e}$ & $0.1456(5)$ & $0.2024(6)$ & $0.3840(5)$ & $4.1(3)$ \\
\hline$P(43)^{e}$ & $0.1331(5)$ & $0.3525(6)$ & $0.4307(6)$ & $4.9(3)$ \\
\hline $\mathrm{P}(51)$ & $0.5561(3)$ & $0.2480(5)$ & $0.6215(3)$ & $6.4(2)$ \\
\hline$P(52)$ & $0.9073(4)$ & $0.1660(5)$ & $0.2924(4)$ & $8.3(2)$ \\
\hline $\mathrm{F}(11)$ & $0.5162(6)$ & $0.3221(9)$ & $0.5985(8)$ & $10.3(5)$ \\
\hline $\mathrm{F}(12)$ & $0.6020(8)$ & $0.277(1)$ & $0.5911(8)$ & $11.7(6)$ \\
\hline $\mathrm{F}(13)$ & $0.5950(7)$ & $0.170(1)$ & $0.6399(9)$ & $15.1(7)$ \\
\hline $\mathrm{F}(14)$ & $0.5894(8)$ & $0.295(1)$ & $0.6753(8)$ & $14.4(7)$ \\
\hline $\mathrm{F}(15)$ & $0.5193(7)$ & $0.209(1)$ & $0.5662(7)$ & $10.4(6)$ \\
\hline $\mathrm{F}(16)$ & $0.5079(7)$ & $0.222(1)$ & $0.6463(7)$ & $11.2(6)$ \\
\hline $\mathrm{F}(21)$ & $0.9564(9)$ & $0.228(1)$ & $0.313(1)$ & $20.2(9)$ \\
\hline $\mathrm{F}(22)$ & $0.8612(7)$ & $0.229(1)$ & $0.2651(10)$ & $12.9(7)$ \\
\hline $\mathrm{F}(23)$ & $0.886(2)$ & $0.191(2)$ & $0.341(1)$ & $19(1)$ \\
\hline $\mathrm{F}(24)$ & $0.915(1)$ & $0.151(2)$ & $0.238(1)$ & $20(1)$ \\
\hline $\mathrm{F}(25)$ & $0.9509(8)$ & $0.097(1)$ & $0.318(1)$ & $19.6(9)$ \\
\hline $\mathrm{F}(26)$ & $0.8547(9)$ & $0.109(1)$ & $0.286(1)$ & $17.8(9)$ \\
\hline $\mathrm{N}(1)$ & $0.2331(8)$ & $-0.1326(9)$ & $0.4472(7)$ & $4.3(4)$ \\
\hline $\mathrm{N}(2)$ & $0.2400(6)$ & $-0.4632(7)$ & $0.4550(5)$ & $1.9(2)$ \\
\hline$C(1)$ & $0.231(1)$ & $-0.065(1)$ & $0.450(1)$ & $5.1(5)$ \\
\hline$C(2)$ & $0.239(1)$ & $0.477(1)$ & $0.4546(10)$ & $4.7(4)$ \\
\hline$C(3)$ & $0.374(1)$ & $0.118(1)$ & $0.499(1)$ & $6.3(6)$ \\
\hline$C(4)$ & $0.377(1)$ & $0.275(1)$ & $0.5352(10)$ & $5.5(6)$ \\
\hline$C(5)$ & $0.0880(9)$ & $0.129(1)$ & $0.4017(9)$ & $4.7(5)$ \\
\hline$C(6)$ & $0.1027(10)$ & $0.287(1)$ & $0.3706(9)$ & $5.1(5)$ \\
\hline$C(11)$ & $0.2355(9)$ & $-0.2180(10)$ & $0.4463(9)$ & $4.0(4)$ \\
\hline$C(12)$ & $0.2228(9)$ & $-0.254(1)$ & $0.4939(9)$ & $4.5(5)$ \\
\hline$C(13)$ & $0.2297(9)$ & $-0.335(1)$ & $0.4965(8)$ & $3.8(4)$ \\
\hline$C(14)$ & $0.2388(9)$ & $-0.3715(10)$ & $0.4556(9)$ & $3.8(4)$ \\
\hline$C(15)$ & $0.2484(8)$ & $-0.337(1)$ & $0.4085(8)$ & $3.2(4)$ \\
\hline$C(16)$ & $0.2428(8)$ & $-0.255(1)$ & $0.4039(8)$ & $3.3(4)$ \\
\hline$C(17)$ & $0.212(1)$ & $-0.210(2)$ & $0.538(1)$ & $9.2(9)$ \\
\hline$C(18)$ & $0.217(1)$ & $-0.380(1)$ & $0.5436(10)$ & $6.0(6)$ \\
\hline$C(19)$ & $0.259(1)$ & $-0.381(1)$ & $0.3633(10)$ & $5.7(6)$ \\
\hline$C(20)$ & $0.2520(9)$ & $-0.210(1)$ & $0.3589(9)$ & $4.5(5)$ \\
\hline$C(21)^{d}$ & $0.3630(6)$ & $-0.0361(9)$ & $0.5374(7)$ & 9.2 \\
\hline$C(22)^{d}$ & $0.3633(7)$ & $-0.067(1)$ & $0.5873(7)$ & 9.2 \\
\hline
\end{tabular}




\begin{tabular}{|c|c|c|c|c|}
\hline$C(23)^{d}$ & $0.3796(9)$ & $-0.145(1)$ & $0.6006(7)$ & 9.2 \\
\hline$C(24)^{d}$ & $0.3957(8)$ & $-0.1916(9)$ & $0.5640(9)$ & 9.2 \\
\hline$C(25)^{d}$ & $0.3953(7)$ & $-0.161(1)$ & $0.5141(8)$ & 9.2 \\
\hline$C(26)^{d}$ & $0.3790(7)$ & $-0.083(1)$ & $0.5008(7)$ & 9.2 \\
\hline$C(27)^{d, e}$ & $0.3687(8)$ & $0.035(1)$ & $0.4171(7)$ & 3.8 \\
\hline $\mathrm{C}(28)^{\mathrm{d}, \mathrm{e}}$ & $0.3321(8)$ & $0.021(1)$ & $0.3620(8)$ & 3.8 \\
\hline$C(29)^{d, e}$ & $0.3587(9)$ & $0.013(1)$ & $0.3221(7)$ & 3.8 \\
\hline $\mathrm{C}(30)^{\mathrm{d}, \mathrm{e}}$ & $0.4219(9)$ & $0.018(1)$ & $0.3373(8)$ & 3.8 \\
\hline $\mathrm{C}(31)^{\mathrm{d}, \mathrm{e}}$ & $0.4584(8)$ & $0.032(2)$ & $0.3924(8)$ & 3.8 \\
\hline $\mathrm{C}(32)^{\mathrm{d}, \mathrm{e}}$ & $0.4319(8)$ & $0.040(1)$ & $0.4322(7)$ & 3.8 \\
\hline$C(33)^{d}$ & $0.0891(8)$ & $-0.032(1)$ & $0.370(1)$ & 15.6 \\
\hline$C(34)^{d}$ & $0.0624(9)$ & $-0.082(2)$ & $0.3967(10)$ & 15.6 \\
\hline$C(35)^{d}$ & $0.041(1)$ & $-0.155(1)$ & $0.374(1)$ & 15.6 \\
\hline$C(36)^{d}$ & $0.047(1)$ & $-0.177(1)$ & $0.324(1)$ & 15.6 \\
\hline$C(37)^{\mathrm{d}}$ & $0.0737(9)$ & $-0.127(2)$ & $0.297(1)$ & 15.6 \\
\hline$C(38)^{d}$ & $0.0948(9)$ & $-0.054(2)$ & $0.320(1)$ & 15.6 \\
\hline$C(39)^{d, e}$ & $0.111(1)$ & $0.023(1)$ & $0.487(1)$ & 6.7 \\
\hline$C(40)^{\mathrm{d}, \mathrm{e}}$ & $0.054(1)$ & $0.045(2)$ & $0.488(1)$ & 6.7 \\
\hline $\mathrm{C}(41)^{\mathrm{d}, \mathrm{e}}$ & $0.040(1)$ & $0.029(2)$ & $0.534(1)$ & 6.7 \\
\hline$C(42) \mathrm{d}, \mathrm{e}$ & $0.082(2)$ & $-0.009(2)$ & $0.579(1)$ & 6.7 \\
\hline$C(43)^{d, e}$ & $0.139(1)$ & $-0.031(2)$ & $0.579(1)$ & 6.7 \\
\hline $\mathrm{C}(44)^{\mathrm{d}, \mathrm{e}}$ & $0.153(1)$ & $-0.015(2)$ & $0.532(1)$ & 6.7 \\
\hline$C(45)^{d, e}$ & $0.1532(10)$ & $0.384(1)$ & $0.3180(8)$ & 4.5 \\
\hline$C(46)^{d, e}$ & $0.2065(9)$ & $0.416(1)$ & $0.3149(9)$ & 4.5 \\
\hline $\mathrm{C}(47)^{\mathrm{d}, \mathrm{e}}$ & $0.211(1)$ & $0.430(2)$ & $0.263(1)$ & 4.5 \\
\hline$C(48) \mathrm{d}, \mathrm{e}$ & $0.161(1)$ & $0.413(1)$ & $0.2151(9)$ & 4.5 \\
\hline$C(49)^{d, e}$ & $0.108(1)$ & $0.382(1)$ & $0.2182(8)$ & 4.5 \\
\hline$C(50)^{\mathrm{d}, \mathrm{e}}$ & $0.1039(9)$ & $0.367(1)$ & $0.2697(9)$ & 4.5 \\
\hline$C(51) \mathrm{d}, \mathrm{e}$ & $0.3260(8)$ & $0.3704(9)$ & $0.5950(6)$ & 2.8 \\
\hline$C(52)^{d, e}$ & $0.2753(7)$ & $0.397(1)$ & $0.6060(7)$ & 2.8 \\
\hline$C(53)^{d, e}$ & $0.2781(9)$ & $0.403(1)$ & $0.6605(8)$ & 2.8 \\
\hline $\mathrm{C}(54)^{\mathrm{d}, \mathrm{e}}$ & $0.332(1)$ & $0.383(1)$ & $0.7041(6)$ & 2.8 \\
\hline$C(55)^{d, e}$ & $0.3823(8)$ & $0.357(1)$ & $0.6931(7)$ & 2.8 \\
\hline$C(56)^{\mathrm{d}, \mathrm{e}}$ & $0.3795(7)$ & $0.351(1)$ & $0.6386(7)$ & 2.8 \\
\hline $\mathrm{C}(57)^{\mathrm{d}, \mathrm{e}}$ & $0.0999(9)$ & $0.451(1)$ & $0.394(1)$ & 5.8 \\
\hline$C(58)^{d, e}$ & $0.087(1)$ & $0.455(1)$ & $0.442(1)$ & 5.8 \\
\hline $\mathrm{C}(59)^{\mathrm{d}, \mathrm{e}}$ & $0.068(1)$ & $0.525(2)$ & $0.458(1)$ & 5.8 \\
\hline $\mathrm{C}(60)^{\mathrm{d}}, \mathrm{e}$ & $0.062(1)$ & $0.592(1)$ & $0.425(1)$ & 5.8 \\
\hline$C(61)^{d, e}$ & $0.075(1)$ & $0.588(1)$ & $0.376(1)$ & 5.8 \\
\hline$C(62)^{d, e}$ & $0.094(1)$ & $0.517(2)$ & $0.3606(10)$ & 5.8 \\
\hline $\mathrm{C}(63)^{\mathrm{d}, \mathrm{e}}$ & $0.3790(8)$ & $0.436(1)$ & $0.5209(8)$ & 3.9 \\
\hline$C(64) \mathrm{d}, \mathrm{e}$ & $0.404(1)$ & $0.475(1)$ & $0.5513(9)$ & 5.2 \\
\hline$C(65)^{d, e}$ & $0.409(1)$ & $0.570(1)$ & $0.5450(10)$ & 3.9 \\
\hline$C(66)^{d, e}$ & $0.423(1)$ & $0.577(1)$ & $0.497(1)$ & 3.9 \\
\hline $\mathrm{C}(67)^{\mathrm{d}, \mathrm{e}}$ & $0.4155(9)$ & $0.514(1)$ & $0.4617(9)$ & 3.9 \\
\hline$C(68)^{d, e}$ & $0.3933(10)$ & $0.443(1)$ & $0.4734(8)$ & 3.9 \\
\hline$C(69)^{d, e}$ & $0.3814(9)$ & $0.442(1)$ & $0.4985(10)$ & 5.2 \\
\hline$C(70)^{d, e}$ & $0.3869(9)$ & $0.499(1)$ & $0.5567(8)$ & 3.9 \\
\hline $\mathrm{C}(71)^{\mathrm{d}, \mathrm{e}}$ & $0.436(1)$ & $0.545(2)$ & $0.560(1)$ & 5.2 \\
\hline $\mathrm{C}(72)^{\mathrm{d}, \mathrm{e}}$ & $0.446(1)$ & $0.582(1)$ & $0.516(1)$ & 5.2 \\
\hline $\mathrm{C}(73)^{\mathrm{d}, \mathrm{e}}$ & $0.424(1)$ & $0.549(1)$ & $0.464(1)$ & 5.2 \\
\hline $\mathrm{C}(74)^{\mathrm{d}, \mathrm{e}}$ & $0.392(1)$ & $0.479(2)$ & $0.4547(9)$ & 5.2 \\
\hline$C(75)^{\mathrm{e}}$ & $0.320(2)$ & $0.099(2)$ & $0.583(1)$ & $2.8(7)$ \\
\hline$C(76)$ & $0.269(1)$ & $0.129(1)$ & $0.5902(9)$ & $5.2(5)$ \\
\hline$C(77)^{\mathrm{e}}$ & $0.277(2)$ & $0.160(3)$ & $0.639(2)$ & $6(1)$ \\
\hline$C(78)^{\mathrm{e}}$ & $0.328(2)$ & $0.165(3)$ & $0.686(2)$ & $5(1)$ \\
\hline$C(79)$ & $0.382(1)$ & $0.134(1)$ & $0.682(1)$ & $5.8(6)$ \\
\hline$C(80)^{e}$ & $0.384(2)$ & $0.105(2)$ & $0.628(1)$ & $3.3(7)$ \\
\hline
\end{tabular}




\begin{tabular}{|c|c|c|c|c|}
\hline $\mathrm{C}(81)^{\mathrm{e}}$ & $0.317(2)$ & $0.165(3)$ & $0.590(2)$ & $4.6(9)$ \\
\hline$C(82)^{e}$ & $0.395(3)$ & $0.166(4)$ & $0.642(2)$ & $8(1)$ \\
\hline$C(83)^{e}$ & $0.324(3)$ & $0.092(5)$ & $0.693(3)$ & $13(1)$ \\
\hline$C(84)^{\mathrm{e}}$ & $0.261(3)$ & $0.103(4)$ & $0.639(3)$ & $10(1)$ \\
\hline$C(85)^{e}$ & $0.104(2)$ & $0.242(2)$ & $0.484(1)$ & $2.9(7)$ \\
\hline$C(86)^{e}$ & $0.044(2)$ & $0.239(3)$ & $0.476(2)$ & $4.2(9)$ \\
\hline$C(87)$ & $0.024(1)$ & $0.269(2)$ & $0.511(1)$ & $8.3(8)$ \\
\hline $\mathrm{C}(88)$ & $0.052(2)$ & $0.288(2)$ & $0.560(2)$ & $11(1)$ \\
\hline$C(89)$ & $0.122(1)$ & $0.289(2)$ & $0.574(1)$ & $9.3(9)$ \\
\hline$C(90)$ & $0.1496(10)$ & $0.274(1)$ & $0.5348(9)$ & $4.9(5)$ \\
\hline $\mathrm{C}(91)^{\mathrm{e}}$ & $0.101(2)$ & $0.301(3)$ & $0.480(2)$ & $6(1)$ \\
\hline$C(92)^{e}$ & $0.037(2)$ & $0.288(2)$ & $0.469(2)$ & $4.5(9)$ \\
\hline$C(93)^{e}$ & $0.362(2)$ & $0.298(2)$ & $0.427(2)$ & $4.2(8)$ \\
\hline$C(94)$ & $0.3212(9)$ & $0.276(1)$ & $0.3727(9)$ & $4.5(5)$ \\
\hline$C(95)$ & $0.334(1)$ & $0.273(2)$ & $0.330(1)$ & $7.7(7)$ \\
\hline$C(96)^{e}$ & $0.412(2)$ & $0.239(3)$ & $0.349(2)$ & $4.7(9)$ \\
\hline $\mathrm{C}(97)$ & $0.446(1)$ & $0.258(2)$ & $0.390(1)$ & $6.2(6)$ \\
\hline $\mathrm{C}(98)^{\mathrm{e}}$ & $0.427(2)$ & $0.290(3)$ & $0.443(2)$ & $5.3(10)$ \\
\hline$C(99)^{e}$ & $0.358(1)$ & $0.237(2)$ & $0.415(1)$ & $1.6(6)$ \\
\hline$C(100)^{e}$ & $0.431(2)$ & $0.226(2)$ & $0.428(2)$ & $3.5(8)$ \\
\hline$C(101)^{e}$ & $0.403(2)$ & $0.282(3)$ & $0.334(2)$ & $4(1)$ \\
\hline$C(102)^{e}$ & $0.133(1)$ & $0.103(2)$ & $0.316(1)$ & $2.2(6)$ \\
\hline C(103) & $0.1895(9)$ & $0.133(1)$ & $0.3120(9)$ & $4.4(5)$ \\
\hline$C(104)^{e}$ & $0.194(2)$ & $0.166(3)$ & $0.264(2)$ & $4.6(10)$ \\
\hline$C(105)^{e}$ & $0.132(2)$ & $0.183(2)$ & $0.221(2)$ & $3.8(8)$ \\
\hline$C(106)$ & $0.081(1)$ & $0.154(2)$ & $0.221(1)$ & $7.5(7)$ \\
\hline$C(107)^{\mathrm{e}}$ & $0.078(2)$ & $0.108(2)$ & $0.273(2)$ & $4.1(8)$ \\
\hline$C(108)^{e}$ & $0.144(2)$ & $0.160(3)$ & $0.319(2)$ & $5.1(10)$ \\
\hline$C(109)^{\mathrm{e}}$ & $0.191(2)$ & $0.115(3)$ & $0.263(2)$ & $5(1)$ \\
\hline$C(110)^{e}$ & $0.120(4)$ & $0.125(5)$ & $0.210(4)$ & $14(1)$ \\
\hline$C(111)^{\mathrm{e}}$ & $0.087(2)$ & $0.157(3)$ & $0.272(2)$ & $5(1)$ \\
\hline$C(112)^{d, e}$ & $0.101(3)$ & $0.455(3)$ & $0.407(4)$ & 28.5 \\
\hline$C(113)^{\mathrm{d}, \mathrm{e}}$ & $0.095(4)$ & $0.484(5)$ & $0.455(3)$ & 28.5 \\
\hline$C(114)^{\mathrm{d}, \mathrm{e}}$ & $0.059(3)$ & $0.477(5)$ & $0.355(3)$ & 28.5 \\
\hline$C(115)^{d, e}$ & $0.012(3)$ & $0.528(5)$ & $0.351(4)$ & 28.5 \\
\hline$C(116)^{\mathrm{d}, \mathrm{e}}$ & $0.006(3)$ & $0.558(4)$ & $0.399(6)$ & 28.5 \\
\hline$C(117)^{\mathrm{d}, \mathrm{e}}$ & $0.048(4)$ & $0.536(5)$ & $0.451(5)$ & 28.5 \\
\hline$C(201)$ & $0.464(3)$ & $-0.041(4)$ & $0.805(3)$ & $20(1)$ \\
\hline$C(202)$ & $0.423(1)$ & $-0.352(2)$ & $0.735(1)$ & $9.3(8)$ \\
\hline$C(203)$ & $0.282(2)$ & $-0.159(4)$ & $0.724(2)$ & $20(1)$ \\
\hline $\mathrm{H}(1)^{\mathrm{d}, \mathrm{e}}$ & $0.240(1)$ & $0.427(2)$ & $0.348(1)$ & 4.5 \\
\hline $\mathrm{H}(2)^{\mathrm{d}, \mathrm{e}}$ & $0.247(1)$ & $0.452(2)$ & $0.261(1)$ & 4.5 \\
\hline $\mathrm{H}(3)^{\mathrm{d}, \mathrm{e}}$ & $0.164(2)$ & $0.423(1)$ & $0.1800(10)$ & 4.5 \\
\hline $\mathrm{H}(4)^{\mathrm{d}, \mathrm{e}}$ & $0.074(1)$ & $0.371(2)$ & $0.1853(9)$ & 4.5 \\
\hline $\mathrm{H}(5)^{\mathrm{d}, \mathrm{e}}$ & $0.068(1)$ & $0.346(2)$ & $0.272(1)$ & 4.5 \\
\hline$H(6)^{d, e}$ & $0.4140(9)$ & $0.333(2)$ & $0.6312(10)$ & 2.8 \\
\hline $\mathrm{H}(7) \mathrm{d}, \mathrm{e}$ & $0.4187(10)$ & $0.344(2)$ & $0.7228(8)$ & 2.8 \\
\hline $\mathrm{H}(8)^{\mathrm{d}, \mathrm{e}}$ & $0.333(1)$ & $0.387(1)$ & $0.7412(7)$ & 2.8 \\
\hline $\mathrm{H}(9)^{\mathrm{d}, \mathrm{e}}$ & $0.244(1)$ & $0.421(2)$ & $0.668(1)$ & 2.8 \\
\hline $\mathrm{H}(10)^{\mathrm{d}, \mathrm{e}}$ & $0.2389(8)$ & $0.410(2)$ & $0.5763(9)$ & 2.8 \\
\hline $\mathrm{H}(11)^{\mathrm{d}, \mathrm{e}}$ & $0.192(1)$ & $-0.029(2)$ & $0.531(2)$ & 6.7 \\
\hline $\mathrm{H}(12)^{\mathrm{d}, \mathrm{e}}$ & $0.167(2)$ & $-0.056(3)$ & $0.610(1)$ & 6.7 \\
\hline $\mathrm{H}(13)^{\mathrm{d}, \mathrm{e}}$ & $0.072(2)$ & $-0.020(2)$ & $0.611(1)$ & 6.7 \\
\hline $\mathrm{H}(14)^{\mathrm{d}, \mathrm{e}}$ & $0.001(1)$ & $0.044(3)$ & $0.535(2)$ & 6.7 \\
\hline $\mathrm{H}(15)^{\mathrm{d}, \mathrm{e}}$ & $0.026(1)$ & $0.070(2)$ & $0.457(1)$ & 6.7 \\
\hline$H(16)^{d}$ & $0.058(1)$ & $-0.067(2)$ & $0.431(1)$ & 15.6 \\
\hline $\mathrm{H}(17)^{\mathrm{d}}$ & $0.023(2)$ & $-0.189(2)$ & $0.392(2)$ & 15.6 \\
\hline $\mathrm{H}(18)^{\mathrm{d}}$ & $0.033(1)$ & $-0.227(1)$ & $0.309(2)$ & 15.6 \\
\hline
\end{tabular}




\begin{tabular}{|c|c|c|c|c|}
\hline$H(19)^{d}$ & $0.078(1)$ & $-0.142(2)$ & $0.264(1)$ & 15.6 \\
\hline $\mathrm{H}(20)^{\mathrm{d}}$ & $0.113(1)$ & $-0.019(2)$ & $0.302(1)$ & 15.6 \\
\hline $\mathrm{H}(21)$ & 0.2831 & -0.1773 & 0.3713 & 7.9 \\
\hline $\mathrm{H}(22)$ & 0.2541 & -0.2472 & 0.3327 & 7.9 \\
\hline $\mathrm{H}(23)$ & 0.2129 & -0.1813 & 0.3410 & 7.9 \\
\hline $\mathrm{H}(24)$ & 0.2309 & -0.4240 & 0.3525 & 6.6 \\
\hline $\mathrm{H}(25)$ & 0.2656 & -0.3546 & 0.3389 & 6.6 \\
\hline $\mathrm{H}(26)$ & 0.3000 & -0.4126 & 0.3858 & 6.6 \\
\hline $\mathrm{H}(27)$ & 0.2126 & -0.3437 & 0.5699 & 8.9 \\
\hline $\mathrm{H}(28)$ & 0.1872 & -0.4153 & 0.5319 & 8.9 \\
\hline $\mathrm{H}(29)$ & 0.2566 & -0.4086 & 0.5654 & 8.9 \\
\hline $\mathrm{H}(30)$ & 0.2481 & -0.2190 & 0.5736 & 9.3 \\
\hline $\mathrm{H}(31)$ & 0.2142 & -0.1532 & 0.5330 & 9.3 \\
\hline $\mathrm{H}(32)$ & 0.1782 & -0.2244 & 0.5423 & 9.3 \\
\hline$H(33)^{d}$ & $0.3524(9)$ & $-0.035(1)$ & $0.6123(9)$ & 9.2 \\
\hline $\mathrm{H}(34)^{\mathrm{d}}$ & $0.380(1)$ & $-0.166(2)$ & $0.6346(9)$ & 9.2 \\
\hline $\mathrm{H}(35)^{\mathrm{d}}$ & $0.407(1)$ & $-0.2446(9)$ & $0.573(1)$ & 9.2 \\
\hline$H(36)^{d}$ & $0.406(1)$ & $-0.193(1)$ & $0.489(1)$ & 9.2 \\
\hline $\mathrm{H}(37)^{\mathrm{d}}$ & $0.379(1)$ & $-0.062(1)$ & $0.4668(7)$ & 9.2 \\
\hline $\mathrm{H}(43)^{\mathrm{d}, \mathrm{e}}$ & $0.377(1)$ & $0.494(2)$ & $0.5891(9)$ & 3.9 \\
\hline $\mathrm{H}(44)^{\mathrm{d}, \mathrm{e}}$ & $0.414(2)$ & $0.613(1)$ & $0.569(1)$ & 3.9 \\
\hline $\mathrm{H}(45)^{\mathrm{d}, \mathrm{e}}$ & $0.438(1)$ & $0.626(1)$ & $0.489(1)$ & 3.9 \\
\hline $\mathrm{H}(46)^{\mathrm{d}, \mathrm{e}}$ & $0.425(1)$ & $0.519(2)$ & $0.4293(10)$ & 3.9 \\
\hline $\mathrm{H}(47)^{\mathrm{d}, \mathrm{e}}$ & $0.388(1)$ & $0.400(1)$ & $0.449(1)$ & 3.9 \\
\hline $\mathrm{H}(48)^{\mathrm{d}, \mathrm{e}}$ & $0.397(1)$ & $0.450(2)$ & $0.581(1)$ & 5.2 \\
\hline $\mathrm{H}(49)^{\mathrm{d}, \mathrm{e}}$ & $0.451(2)$ & $0.568(2)$ & $0.596(1)$ & 5.2 \\
\hline $\mathrm{H}(50)^{\mathrm{d}, \mathrm{e}}$ & $0.468(2)$ & $0.630(1)$ & $0.522(2)$ & 5.2 \\
\hline $\mathrm{H}(51)^{\mathrm{d}, \mathrm{e}}$ & $0.431(2)$ & $0.574(2)$ & $0.434(1)$ & 5.2 \\
\hline $\mathrm{H}(52)^{\mathrm{d}, \mathrm{e}}$ & $0.376(2)$ & $0.457(2)$ & $0.4188(9)$ & 5.2 \\
\hline $\mathrm{H}(53)^{\mathrm{d}, \mathrm{e}}$ & $0.092(2)$ & $0.410(2)$ & $0.464(1)$ & 5.8 \\
\hline$H(54)^{d, e}$ & $0.060(1)$ & $0.528(2)$ & $0.491(1)$ & 5.8 \\
\hline $\mathrm{H}(55)^{\mathrm{d}, \mathrm{e}}$ & $0.049(2)$ & $0.640(2)$ & $0.435(2)$ & 5.8 \\
\hline $\mathrm{H}(56)^{\mathrm{d}, \mathrm{e}}$ & $0.070(2)$ & $0.633(2)$ & $0.354(2)$ & 5.8 \\
\hline $\mathrm{H}(57)^{\mathrm{d}, \mathrm{e}}$ & $0.102(1)$ & $0.514(2)$ & $0.328(1)$ & 5.8 \\
\hline $\mathrm{H}(58)^{\mathrm{d}, \mathrm{e}}$ & $0.4568(9)$ & $0.050(2)$ & $0.4698(8)$ & 3.8 \\
\hline $\mathrm{H}(59)^{\mathrm{d}, \mathrm{e}}$ & $0.5015(8)$ & $0.036(2)$ & $0.4027(10)$ & 3.8 \\
\hline $\mathrm{H}(60)^{\mathrm{d}, \mathrm{e}}$ & $0.440(1)$ & $0.012(2)$ & $0.3101(8)$ & 3.8 \\
\hline $\mathrm{H}(61)^{\mathrm{d}, \mathrm{e}}$ & $0.3338(10)$ & $0.003(2)$ & $0.2846(7)$ & 3.8 \\
\hline $\mathrm{H}(62)^{\mathrm{d}, \mathrm{e}}$ & $0.2891(8)$ & $0.017(2)$ & $0.3516(9)$ & 3.8 \\
\hline $\mathrm{H}(63)^{\mathrm{d}, \mathrm{e}}$ & $0.063(5)$ & $0.457(7)$ & $0.322(4)$ & 28.5 \\
\hline $\mathrm{H}(64)^{\mathrm{d}, \mathrm{e}}$ & $-0.017(4)$ & $0.543(7)$ & $0.316(5)$ & 28.5 \\
\hline $\mathrm{H}(65)^{\mathrm{d}, \mathrm{e}}$ & $-0.026(4)$ & $0.593(5)$ & $0.397(8)$ & 28.5 \\
\hline $\mathrm{H}(66)^{\mathrm{d}, \mathrm{e}}$ & $0.044(6)$ & $0.556(7)$ & $0.484(5)$ & 28.5 \\
\hline $\mathrm{H}(67)^{\mathrm{d}, \mathrm{e}}$ & $0.123(5)$ & $0.469(7)$ & $0.490(4)$ & 28.5 \\
\hline
\end{tabular}

a Estimated standard deviations are given in parentheses. ${ }^{b}$ The $\mathrm{Pt}, \mathrm{Cl}, \mathrm{P}$, and $\mathrm{F}$ atoms were assigned anisotropic thermal parameters given as the isotropic equivalent displacement parameter defined as $B_{\text {eq }}=\left(8 \pi^{2} / 3\right)\left(U_{11}\left(a a^{*}\right)^{2}+U_{22}\left(b b^{*}\right)^{2}+U_{33}\left(c c^{*}\right)^{2}+2 U_{12} a a^{*} b b^{*} \cos \gamma+2 U_{13} a a^{*} c c^{*} \cos \beta+\right.$ $\left.2 U_{23} b b^{*} c c^{*} \cos \alpha\right)$. Other non-hydrogen atoms were refined isotropically. ${ }^{\mathrm{c}}$ The position of all hydrogen atoms were calculated with $\mathrm{C}-\mathrm{H}=0.95 \AA$, and were not refined with the appropriate $B$ iso or refined as phenyl rigid groups. $\mathrm{d}_{\text {Treated as }} \mathrm{C}_{6} \mathrm{H}_{5}$ rigid groups. ${ }^{\mathrm{e}}$ Disordered with 0.5 occupancy.

Table S6-3. Final Anisotropic Thermal Parameters for $7 \mathbf{a} \cdot 3 \mathrm{CH}_{2} \mathrm{Cl}_{2}{ }^{\mathrm{a}, \mathrm{b}}$

\begin{tabular}{lcccccc}
\hline atom & $U_{11}$ & $U_{22}$ & $U_{33}$ & $U_{12}$ & $U_{13}$ & $U_{23}$ \\
\hline $\operatorname{Pt}(1)$ & $0.0504(4)$ & $0.0152(3)$ & $0.0402(4)$ & $0.0037(4)$ & $0.0111(3)$ & $-0.0010(3)$
\end{tabular}




\begin{tabular}{|c|c|c|c|c|c|c|}
\hline $\operatorname{Pt}(2)$ & $0.0374(3)$ & $0.0149(2)$ & $0.0323(3)$ & $0.0023(4)$ & $0.0104(3)$ & $-0.0008(3)$ \\
\hline $\operatorname{Pt}(3)$ & $0.0539(5)$ & $0.0149(3)$ & $0.0427(4)$ & $0.0032(4)$ & $0.0133(4)$ & $0.0005(3)$ \\
\hline $\mathrm{Cl}(1)$ & $0.191(9)$ & $0.160(9)$ & $0.141(8)$ & $-0.024(8)$ & $0.061(7)$ & $-0.044(7)$ \\
\hline $\mathrm{Cl}(2)$ & $0.167(9)$ & $0.22(1)$ & $0.141(8)$ & $-0.021(8)$ & $0.038(7)$ & $0.081(7)$ \\
\hline $\mathrm{Cl}(3)$ & $0.29(1)$ & $0.22(1)$ & $0.20(1)$ & $0.01(1)$ & $0.06(1)$ & $0.06(1)$ \\
\hline $\mathrm{Cl}(4)$ & $0.52(2)$ & $0.26(2)$ & $0.20(1)$ & $0.14(2)$ & $0.20(1)$ & $0.08(1)$ \\
\hline $\mathrm{Cl}(5)$ & $0.26(2)$ & $0.35(2)$ & $0.16(1)$ & $0.03(2)$ & $0.02(1)$ & $-0.01(1)$ \\
\hline $\mathrm{Cl}(6)$ & $0.225(10)$ & $0.30(2)$ & $0.36(1)$ & $-0.05(1)$ & $0.219(9)$ & $-0.09(1)$ \\
\hline $\mathrm{P}(11)$ & $0.024(5)$ & $0.023(4)$ & $0.019(4)$ & $0.009(4)$ & $0.013(4)$ & $0.001(3)$ \\
\hline$P(12)$ & $0.027(4)$ & $0.015(3)$ & $0.015(4)$ & $0.006(4)$ & $0.014(3)$ & $0.001(3)$ \\
\hline$P(13)$ & $0.048(6)$ & $0.014(4)$ & $0.017(4)$ & $-0.013(4)$ & $0.014(4)$ & $-0.008(3)$ \\
\hline$P(21)$ & $0.054(7)$ & $0.037(6)$ & $0.056(7)$ & $0.002(5)$ & $0.016(6)$ & $-0.001(5)$ \\
\hline $\mathrm{P}(22)$ & $0.031(5)$ & $0.030(5)$ & $0.058(7)$ & $0.015(5)$ & $0.005(5)$ & $0.004(5)$ \\
\hline $\mathrm{P}(23)$ & $0.084(9)$ & $0.017(5)$ & $0.071(8)$ & $-0.017(5)$ & $0.017(7)$ & $0.003(5)$ \\
\hline $\mathrm{P}(31)$ & $0.028(5)$ & $0.019(4)$ & $0.021(5)$ & $-0.008(4)$ & $-0.005(4)$ & $-0.013(3)$ \\
\hline $\mathrm{P}(32)$ & $0.032(4)$ & $0.014(4)$ & $0.019(4)$ & $0.004(4)$ & $0.017(3)$ & $-0.001(3)$ \\
\hline $\mathrm{P}(33)$ & $0.042(6)$ & $0.019(4)$ & $0.021(4)$ & $0.010(4)$ & $0.012(4)$ & $0.002(4)$ \\
\hline $\mathrm{P}(41)$ & $0.053(7)$ & $0.037(6)$ & $0.051(7)$ & $0.000(5)$ & $0.006(6)$ & $-0.006(5)$ \\
\hline $\mathrm{P}(42)$ & $0.062(7)$ & $0.034(5)$ & $0.055(7)$ & $0.012(6)$ & $0.016(6)$ & $0.010(5)$ \\
\hline$P(43)$ & $0.054(7)$ & $0.025(5)$ & $0.11(1)$ & $0.018(5)$ & $0.027(7)$ & $-0.004(6)$ \\
\hline$P(51)$ & $0.051(4)$ & $0.104(5)$ & $0.082(5)$ & $0.002(4)$ & $0.014(4)$ & $0.012(4)$ \\
\hline$P(52)$ & $0.066(5)$ & $0.106(6)$ & $0.115(7)$ & $-0.002(5)$ & $0.000(5)$ & $0.039(5)$ \\
\hline $\mathrm{F}(11)$ & $0.051(9)$ & $0.08(1)$ & $0.22(2)$ & $0.017(8)$ & $0.00(1)$ & $0.00(1)$ \\
\hline $\mathrm{F}(12)$ & $0.11(1)$ & $0.17(2)$ & $0.17(2)$ & $0.01(1)$ & $0.05(1)$ & $0.08(1)$ \\
\hline $\mathrm{F}(13)$ & $0.06(1)$ & $0.23(2)$ & $0.27(2)$ & $0.05(1)$ & $0.05(1)$ & $0.15(2)$ \\
\hline $\mathrm{F}(14)$ & $0.11(1)$ & $0.25(2)$ & $0.12(2)$ & $0.00(1)$ & $-0.04(1)$ & $-0.08(1)$ \\
\hline $\mathrm{F}(15)$ & $0.10(1)$ & $0.17(2)$ & $0.11(1)$ & $0.01(1)$ & $0.02(1)$ & $-0.02(1)$ \\
\hline $\mathrm{F}(16)$ & $0.067(9)$ & $0.23(2)$ & $0.12(1)$ & $-0.03(1)$ & $0.020(9)$ & $0.05(1)$ \\
\hline $\mathrm{F}(21)$ & $0.10(1)$ & $0.25(3)$ & $0.32(3)$ & $-0.07(1)$ & $-0.06(2)$ & $0.11(2)$ \\
\hline $\mathrm{F}(22)$ & $0.07(1)$ & $0.18(2)$ & $0.22(2)$ & $0.02(1)$ & $0.03(1)$ & $0.05(2)$ \\
\hline $\mathrm{F}(23)$ & $0.35(4)$ & $0.23(3)$ & $0.14(2)$ & $0.01(3)$ & $0.08(2)$ & $0.06(2)$ \\
\hline $\mathrm{F}(24)$ & $0.35(3)$ & $0.24(3)$ & $0.19(2)$ & $0.12(2)$ & $0.12(2)$ & $0.06(2)$ \\
\hline $\mathrm{F}(25)$ & $0.09(1)$ & $0.13(2)$ & $0.45(4)$ & $0.00(1)$ & $0.00(2)$ & $0.07(2)$ \\
\hline$F(26)$ & $0.10(1)$ & $0.21(2)$ & $0.36(3)$ & $0.08(1)$ & $0.07(2)$ & $0.12(2)$ \\
\hline
\end{tabular}

$\mathrm{a}$ Estmated standard deviations are given in parentheses. $\mathrm{b}$ Parameters have the form $\exp (-$ $\left.2 \pi^{2}\left(U_{11} h^{2} a^{* 2}+\cdots+2 U_{12} h k a^{*} b^{*} \cos \gamma+\cdots\right)\right)$

Table S6-4. Bond Lengths for Non-hydrogen Atoms of $7 \mathbf{a} \cdot 3 \mathrm{CH}_{2} \mathrm{Cl}_{2}{ }^{\mathrm{a}}$

\begin{tabular}{|c|c|c|c|c|c|}
\hline atom & atom & distance & atom & atom & distance \\
\hline$\overline{\operatorname{Pt}(1)}$ & $\operatorname{Pt}(2)$ & $2.7020(8)$ & $\mathrm{P}(11)$ & $C(21)$ & $1.79(2)$ \\
\hline $\operatorname{Pt}(1)$ & $\mathrm{P}(11)$ & $2.217(7)$ & $P(12)$ & $P(22)$ & $1.48(2)$ \\
\hline $\operatorname{Pt}(1)$ & $\mathrm{P}(21)$ & $2.39(1)$ & $P(12)$ & $P(23)$ & $2.49(1)$ \\
\hline $\operatorname{Pt}(1)$ & $\mathrm{P}(31)$ & $2.319(8)$ & $P(12)$ & $C(3)$ & $1.73(2)$ \\
\hline $\operatorname{Pt}(1)$ & $\mathrm{P}(41)$ & $2.21(1)$ & $P(12)$ & $C(4)$ & $1.97(2)$ \\
\hline $\operatorname{Pt}(1)$ & $C(1)$ & $1.93(2)$ & $P(12)$ & $C(93)$ & $2.19(5)$ \\
\hline $\operatorname{Pt}(2)$ & $\operatorname{Pt}(3)$ & $2.6966(8)$ & $\mathrm{P}(12)$ & C(99) & $1.82(4)$ \\
\hline $\operatorname{Pt}(2)$ & $\mathrm{P}(12)$ & $2.271(8)$ & $P(13)$ & $\mathrm{P}(23)$ & $1.30(2)$ \\
\hline $\operatorname{Pt}(2)$ & $\mathrm{P}(22)$ & $2.300(9)$ & $\mathrm{P}(13)$ & $C(4)$ & $1.71(2)$ \\
\hline $\operatorname{Pt}(2)$ & $\mathrm{P}(32)$ & $2.255(9)$ & $\mathrm{P}(13)$ & $C(51)$ & $1.81(2)$ \\
\hline $\operatorname{Pt}(2)$ & $\mathrm{P}(42)$ & $2.21(1)$ & $\mathrm{P}(13)$ & $C(63)$ & $1.84(2)$ \\
\hline $\operatorname{Pt}(3)$ & $P(13)$ & $2.313(8)$ & $P(21)$ & $C(3)$ & $1.42(3)$ \\
\hline $\operatorname{Pt}(3)$ & $\mathrm{P}(23)$ & $2.24(1)$ & $\mathrm{P}(21)$ & $C(21)$ & $2.15(2)$ \\
\hline $\operatorname{Pt}(3)$ & $\mathrm{P}(33)$ & $2.282(8)$ & $P(21)$ & $C(27)$ & $1.81(3)$ \\
\hline $\operatorname{Pt}(3)$ & $\mathrm{P}(43)$ & $2.29(1)$ & $\mathrm{P}(22)$ & $C(3)$ & $2.04(3)$ \\
\hline $\operatorname{Pt}(3)$ & $C(2)$ & $1.98(2)$ & $\mathrm{P}(22)$ & $C(4)$ & $1.72(2)$ \\
\hline $\mathrm{Cl}(1)$ & $C(202)$ & $1.73(3)$ & $\mathrm{P}(22)$ & $C(81)$ & $1.86(5)$ \\
\hline
\end{tabular}




\begin{tabular}{|c|c|c|c|c|c|}
\hline $\mathrm{Cl}(2)$ & $C(202)$ & $1.76(4)$ & $\mathrm{P}(23)$ & $C(4)$ & $1.88(2)$ \\
\hline $\mathrm{Cl}(3)$ & $C(201)$ & $1.76(7)$ & $\mathrm{P}(23)$ & $C(93)$ & $1.90(5)$ \\
\hline $\mathrm{Cl}(4)$ & $C(201)$ & $1.66(7)$ & $\mathrm{P}(23)$ & $C(69)$ & $1.85(2)$ \\
\hline $\mathrm{Cl}(5)$ & $C(203)$ & $1.72(7)$ & $\mathrm{P}(23)$ & $C(63)$ & $1.87(2)$ \\
\hline $\mathrm{Cl}(6)$ & $C(203)$ & $1.79(6)$ & $\mathrm{P}(23)$ & C(68) & $2.11(3)$ \\
\hline $\mathrm{P}(11)$ & $\mathrm{P}(21)$ & $1.38(2)$ & $\mathrm{P}(31)$ & $\mathrm{P}(41)$ & $1.35(2)$ \\
\hline $\mathrm{P}(11)$ & $\mathrm{P}(22)$ & $2.49(1)$ & $\mathrm{P}(31)$ & $\mathrm{P}(42)$ & $2.43(1)$ \\
\hline $\mathrm{P}(11)$ & $C(3)$ & $1.84(3)$ & $\mathrm{P}(31)$ & C(5) & $1.85(3)$ \\
\hline $\mathrm{P}(11)$ & $C(75)$ & $1.88(4)$ & $\mathrm{P}(31)$ & $C(102)$ & $1.79(4)$ \\
\hline $\mathrm{P}(31)$ & $C(33)$ & $1.90(2)$ & $\mathrm{P}(51)$ & $\mathrm{F}(15)$ & $1.53(2)$ \\
\hline $\mathrm{P}(32)$ & $\mathrm{P}(42)$ & $1.41(2)$ & $\mathrm{P}(51)$ & $\mathrm{F}(16)$ & $1.56(2)$ \\
\hline $\mathrm{P}(32)$ & $\mathrm{P}(43)$ & $2.41(1)$ & $P(52)$ & $\mathrm{F}(21)$ & $1.51(2)$ \\
\hline $\mathrm{P}(32)$ & $C(5)$ & $1.77(2)$ & $\mathrm{P}(52)$ & $\mathrm{F}(22)$ & $1.51(2)$ \\
\hline $\mathrm{P}(32)$ & $C(6)$ & $1.99(2)$ & $\mathrm{P}(52)$ & $\mathrm{F}(23)$ & $1.58(4)$ \\
\hline $\mathrm{P}(32)$ & $C(85)$ & $1.78(4)$ & $\mathrm{P}(52)$ & $\mathrm{F}(24)$ & $1.50(4)$ \\
\hline $\mathrm{P}(33)$ & $\mathrm{P}(43)$ & $1.38(2)$ & $\mathrm{P}(52)$ & $\mathrm{F}(25)$ & $1.55(2)$ \\
\hline $\mathrm{P}(33)$ & $C(6)$ & $1.64(2)$ & $\mathrm{P}(52)$ & $\mathrm{F}(26)$ & $1.54(2)$ \\
\hline $\mathrm{P}(33)$ & $C(45)$ & $1.80(2)$ & $\mathrm{N}(1)$ & $C(1)$ & $1.16(3)$ \\
\hline $\mathrm{P}(33)$ & C(57) & $1.90(3)$ & $\mathrm{N}(1)$ & $C(11)$ & $1.47(2)$ \\
\hline $\mathrm{P}(33)$ & $C(112)$ & $2.08(8)$ & $\mathrm{N}(2)$ & $\mathrm{C}(2)^{*}$ & $1.02(2)$ \\
\hline $\mathrm{P}(41)$ & $C(5)$ & $1.73(2)$ & $\mathrm{N}(2)$ & $C(14)$ & $1.57(2)$ \\
\hline $\mathrm{P}(41)$ & C(33) & $2.08(2)$ & $C(11)$ & $C(12)$ & $1.50(3)$ \\
\hline $\mathrm{P}(41)$ & C(39) & $1.76(3)$ & $C(11)$ & $C(16)$ & $1.33(3)$ \\
\hline $\mathrm{P}(42)$ & $C(5)$ & $2.02(3)$ & $C(12)$ & $C(13)$ & $1.39(3)$ \\
\hline $\mathrm{P}(42)$ & $C(6)$ & $1.73(2)$ & $C(12)$ & $\mathrm{C}(17)$ & $1.46(4)$ \\
\hline $\mathrm{P}(42)$ & $C(108)$ & $1.81(5)$ & $C(13)$ & $C(14)$ & $1.32(3)$ \\
\hline $\mathrm{P}(43)$ & $C(6)$ & $1.84(2)$ & $C(13)$ & $C(18)$ & $1.56(4)$ \\
\hline $\mathrm{P}(43)$ & $\mathrm{C}(91)$ & $1.92(6)$ & $C(14)$ & $C(15)$ & $1.45(3)$ \\
\hline $\mathrm{P}(43)$ & $C(57)$ & $1.96(2)$ & $C(15)$ & $C(16)$ & $1.42(3)$ \\
\hline $\mathrm{P}(43)$ & C(58) & $2.14(3)$ & $C(15)$ & C(19) & $1.49(3)$ \\
\hline $\mathrm{P}(43)$ & $C(112)$ & $1.92(6)$ & $C(16)$ & $C(20)$ & $1.47(3)$ \\
\hline $\mathrm{P}(51)$ & $\mathrm{F}(11)$ & $1.56(2)$ & $C(75)$ & $C(76)$ & $1.38(5)$ \\
\hline $\mathrm{P}(51)$ & $\mathrm{F}(12)$ & $1.63(2)$ & $\mathrm{C}(75)$ & $C(80)$ & $1.53(4)$ \\
\hline $\mathrm{P}(51)$ & $\mathrm{F}(13)$ & $1.59(2)$ & $\mathrm{C}(75)$ & $\mathrm{C}(81)$ & $1.14(6)$ \\
\hline $\mathrm{P}(51)$ & $\mathrm{F}(14)$ & $1.55(2)$ & $C(76)$ & $\mathrm{C}(77)$ & $1.31(6)$ \\
\hline$C(76)$ & $C(81)$ & $1.29(5)$ & $C(91)$ & $C(92)$ & $1.45(7)$ \\
\hline$C(76)$ & $C(84)$ & $1.41(9)$ & $C(93)$ & C(94) & $1.44(4)$ \\
\hline $\mathrm{C}(77)$ & $C(78)$ & $1.38(6)$ & $C(93)$ & $C(98)$ & $1.44(6)$ \\
\hline C(77) & $C(81)$ & $1.82(8)$ & $C(93)$ & C(99) & $1.09(5)$ \\
\hline C(77) & $C(84)$ & $1.04(9)$ & C(94) & C(95) & $1.25(4)$ \\
\hline$C(78)$ & $C(79)$ & $1.41(6)$ & $C(94)$ & C(99) & $1.31(3)$ \\
\hline C(78) & $C(83)$ & $1.27(9)$ & C(95) & $C(96)$ & $1.81(5)$ \\
\hline$C(79)$ & $C(80)$ & $1.52(5)$ & C(95) & $C(101)$ & $1.59(6)$ \\
\hline$C(79)$ & $C(82)$ & $1.30(8)$ & $C(96)$ & $\mathrm{C}(97)$ & $1.11(5)$ \\
\hline C(79) & $C(83)$ & $1.66(9)$ & $C(96)$ & $C(101)$ & $0.83(6)$ \\
\hline$C(80)$ & $C(81)$ & $1.84(5)$ & C(97) & $C(98)$ & $1.68(6)$ \\
\hline$C(80)$ & $C(82)$ & $1.11(7)$ & $\mathrm{C}(97)$ & $C(100)$ & $1.30(5)$ \\
\hline C(81) & $C(82)$ & $1.84(6)$ & C(97) & $C(101)$ & $1.50(5)$ \\
\hline C(83) & $C(84)$ & $1.64(9)$ & C(98) & C(99) & $1.77(5)$ \\
\hline C(85) & $C(86)$ & $1.34(6)$ & $C(98)$ & $C(100)$ & $1.16(6)$ \\
\hline C(85) & $C(90)$ & $1.47(4)$ & C(99) & $C(100)$ & $1.62(5)$ \\
\hline C(85) & $C(91)$ & $1.01(6)$ & $C(102)$ & $C(103)$ & $1.46(4)$ \\
\hline C(85) & $C(92)$ & $1.67(6)$ & $C(102)$ & $C(107)$ & $1.38(4)$ \\
\hline$C(86)$ & C(87) & $1.28(6)$ & $C(102)$ & $C(108)$ & $1.02(6)$ \\
\hline$C(86)$ & $C(91)$ & $1.68(7)$ & $C(102)$ & $C(111)$ & $1.56(5)$ \\
\hline$C(86)$ & $C(92)$ & $0.86(6)$ & $C(103)$ & $C(104)$ & $1.40(6)$ \\
\hline
\end{tabular}




\begin{tabular}{|c|c|c|c|c|c|}
\hline$C(87)$ & $C(88)$ & $1.24(5)$ & $C(103)$ & $C(108)$ & $1.24(6)$ \\
\hline C (87) & C(92) & $1.29(6)$ & $C(103)$ & C(109) & $1.31(6)$ \\
\hline C(88) & C(89) & $1.55(5)$ & $C(104)$ & $C(105)$ & $1.52(5)$ \\
\hline C(89) & $C(90)$ & $1.42(5)$ & $C(104)$ & $C(109)$ & $0.87(7)$ \\
\hline$C(90)$ & $C(91)$ & $1.53(5)$ & $C(104)$ & $C(110)$ & $1.94(9)$ \\
\hline$C(105)$ & $C(106)$ & $1.30(5)$ & $C(30)$ & $C(29)$ & $1.40(3)$ \\
\hline$C(105)$ & $C(109)$ & $1.84(6)$ & C(29) & C(28) & $1.40(3)$ \\
\hline$C(105)$ & $C(110)$ & $1.0(1)$ & $C(39)$ & $C(44)$ & $1.40(3)$ \\
\hline$C(106)$ & $C(107)$ & $1.60(6)$ & C(39) & $C(40)$ & $1.40(4)$ \\
\hline$C(106)$ & $C(110)$ & $1.2(1)$ & $C(44)$ & $C(43)$ & $1.40(5)$ \\
\hline$C(106)$ & $C(111)$ & $1.29(6)$ & $C(43)$ & $C(42)$ & $1.40(5)$ \\
\hline$C(107)$ & $C(108)$ & $1.83(5)$ & $C(42)$ & $\mathrm{C}(41)$ & $1.40(4)$ \\
\hline$C(107)$ & $C(111)$ & $0.87(6)$ & $C(41)$ & $C(40)$ & $1.40(5)$ \\
\hline$C(108)$ & $C(111)$ & $1.45(5)$ & $C(45)$ & $C(46)$ & $1.40(3)$ \\
\hline$C(109)$ & $C(110)$ & $1.75(9)$ & $C(45)$ & $C(50)$ & $1.40(3)$ \\
\hline$C(21)$ & $C(22)$ & $1.40(3)$ & $C(46)$ & $\mathrm{C}(47)$ & $1.40(4)$ \\
\hline$C(21)$ & $C(26)$ & $1.40(3)$ & $\mathrm{C}(47)$ & $C(48)$ & $1.40(3)$ \\
\hline$C(22)$ & $C(23)$ & $1.40(3)$ & $C(48)$ & $C(49)$ & $1.40(4)$ \\
\hline$C(23)$ & $C(24)$ & $1.40(3)$ & $\mathrm{C}(49)$ & $C(50)$ & $1.40(3)$ \\
\hline$C(24)$ & $C(25)$ & $1.40(3)$ & $C(51)$ & $C(52)$ & $1.40(3)$ \\
\hline$C(25)$ & $C(26)$ & $1.40(3)$ & $C(51)$ & $C(56)$ & $1.40(2)$ \\
\hline$C(33)$ & $C(34)$ & $1.40(4)$ & $C(52)$ & $C(53)$ & $1.40(3)$ \\
\hline C(33) & $C(38)$ & $1.40(4)$ & $C(53)$ & $C(54)$ & $1.40(2)$ \\
\hline$C(34)$ & $C(35)$ & $1.40(4)$ & $C(54)$ & $C(55)$ & $1.40(3)$ \\
\hline C(35) & $C(36)$ & $1.40(5)$ & $C(55)$ & $C(56)$ & $1.40(3)$ \\
\hline$C(36)$ & $C(37)$ & $1.40(4)$ & $C(69)$ & $C(64)$ & $1.40(3)$ \\
\hline$C(37)$ & $C(38)$ & $1.40(4)$ & $C(69)$ & $C(74)$ & $1.40(4)$ \\
\hline$C(27)$ & $C(32)$ & $1.40(3)$ & $C(69)$ & $C(63)$ & $0.61(4)$ \\
\hline$C(27)$ & $C(28)$ & $1.40(2)$ & $C(69)$ & $C(70)$ & $1.76(3)$ \\
\hline$C(32)$ & $C(31)$ & $1.40(3)$ & $C(69)$ & $C(68)$ & $0.80(4)$ \\
\hline$C(31)$ & $C(30)$ & $1.40(3)$ & $C(64)$ & $C(71)$ & $1.40(4)$ \\
\hline C(64) & $C(63)$ & $1.04(3)$ & $C(57)$ & $C(113)$ & $1.7(1)$ \\
\hline$C(64)$ & $C(70)$ & $0.62(3)$ & $C(62)$ & $C(61)$ & $1.40(4)$ \\
\hline$C(64)$ & $C(65)$ & $1.64(3)$ & $C(62)$ & $C(112)$ & $1.57(9)$ \\
\hline $\mathrm{C}(71)$ & $C(72)$ & $1.40(4)$ & $C(62)$ & $C(114)$ & $1.04(8)$ \\
\hline $\mathrm{C}(71)$ & $C(70)$ & $1.37(4)$ & $C(61)$ & $C(60)$ & $1.40(5)$ \\
\hline $\mathrm{C}(71)$ & $C(65)$ & $0.75(3)$ & $C(61)$ & $C(115)$ & $1.72(8)$ \\
\hline $\mathrm{C}(71)$ & $C(66)$ & $1.64(4)$ & $C(60)$ & C(59) & $1.40(4)$ \\
\hline $\mathrm{C}(72)$ & $\mathrm{C}(73)$ & $1.40(4)$ & $C(60)$ & $C(116)$ & $1.37(7)$ \\
\hline $\mathrm{C}(72)$ & $C(65)$ & $1.35(5)$ & $C(60)$ & $C(117)$ & $1.3(1)$ \\
\hline $\mathrm{C}(72)$ & $C(66)$ & $0.59(3)$ & $C(59)$ & $C(58)$ & $1.40(4)$ \\
\hline $\mathrm{C}(72)$ & $C(67)$ & $1.78(3)$ & $C(59)$ & $C(116)$ & $1.77(9)$ \\
\hline$C(73)$ & $C(74)$ & $1.40(4)$ & $C(59)$ & $C(117)$ & $0.48(9)$ \\
\hline C(73) & $C(66)$ & $1.01(4)$ & $C(59)$ & $C(113)$ & $0.97(9)$ \\
\hline C(73) & $C(67)$ & $0.63(3)$ & $C(58)$ & $C(112)$ & $1.1(1)$ \\
\hline $\mathrm{C}(74)$ & $C(67)$ & $0.80(3)$ & $C(58)$ & $C(117)$ & $1.7(1)$ \\
\hline $\mathrm{C}(74)$ & $C(68)$ & $0.77(3)$ & $C(58)$ & $C(113)$ & $0.59(8)$ \\
\hline$C(63)$ & $C(70)$ & $1.40(3)$ & $C(112)$ & $C(114)$ & $1.4(1)$ \\
\hline$C(63)$ & $C(68)$ & $1.40(3)$ & $C(112)$ & $C(113)$ & $1.4(1)$ \\
\hline C(70) & $C(65)$ & $1.40(3)$ & $C(114)$ & $C(115)$ & $1.4(1)$ \\
\hline$C(65)$ & $C(66)$ & $1.40(4)$ & $C(115)$ & $C(116)$ & $1.4(2)$ \\
\hline$C(66)$ & $C(67)$ & $1.40(3)$ & $C(116)$ & $C(117)$ & $1.4(1)$ \\
\hline$C(67)$ & $C(68)$ & $1.40(3)$ & $C(117)$ & $C(113)$ & $1.4(1)$ \\
\hline$C(57)$ & $C(62)$ & $1.40(4)$ & & & \\
\hline C(57) & $C(58)$ & $1.40(4)$ & & & \\
\hline C(57) & $C(112)$ & $0.3(1)$ & & & \\
\hline
\end{tabular}


C(57) C(114)

1.19(7)

a Estimated standard deviations are given in parentheses.

Table S6-5. Bond Angles for Non-hydrogen Atoms of $7 \mathbf{a} \cdot 3 \mathrm{CH}_{2} \mathrm{Cl}_{2}{ }^{\mathrm{a}}$

\begin{tabular}{|c|c|c|c|c|c|c|c|}
\hline atom & atom & atom & angle & atom & atom & atom & angle \\
\hline$\overline{\operatorname{Pt}(2)}$ & $\operatorname{Pt}(1)$ & $\mathrm{P}(11)$ & $85.3(2)$ & $\mathrm{P}(12)$ & $\operatorname{Pt}(2)$ & $\mathrm{P}(42)$ & $142.9(4)$ \\
\hline $\operatorname{Pt}(2)$ & $\operatorname{Pt}(1)$ & $\mathrm{P}(21)$ & $88.0(3)$ & $\mathrm{P}(22)$ & $\operatorname{Pt}(2)$ & $\mathrm{P}(32)$ & $142.7(4)$ \\
\hline $\operatorname{Pt}(2)$ & $\operatorname{Pt}(1)$ & $\mathrm{P}(31)$ & $85.8(2)$ & $\mathrm{P}(22)$ & $\operatorname{Pt}(2)$ & $\mathrm{P}(42)$ & $176.3(4)$ \\
\hline $\operatorname{Pt}(2)$ & $\operatorname{Pt}(1)$ & $\mathrm{P}(41)$ & $89.5(3)$ & $\mathrm{P}(32)$ & $\operatorname{Pt}(2)$ & $P(42)$ & $36.9(4)$ \\
\hline $\operatorname{Pt}(2)$ & $\operatorname{Pt}(1)$ & $C(1)$ & 176.7(9) & $\operatorname{Pt}(2)$ & $\operatorname{Pt}(3)$ & $P(13)$ & $88.6(2)$ \\
\hline $\mathrm{P}(11)$ & $\operatorname{Pt}(1)$ & $P(21)$ & $34.7(4)$ & $\operatorname{Pt}(2)$ & $\operatorname{Pt}(3)$ & $P(23)$ & $85.5(3)$ \\
\hline $\mathrm{P}(11)$ & $\operatorname{Pt}(1)$ & $\mathrm{P}(31)$ & 170.6(3) & $\operatorname{Pt}(2)$ & $\operatorname{Pt}(3)$ & $P(33)$ & $89.7(2)$ \\
\hline$P(11)$ & $\operatorname{Pt}(1)$ & $\mathrm{P}(41)$ & $142.2(4)$ & $\operatorname{Pt}(2)$ & $\operatorname{Pt}(3)$ & $P(43)$ & $85.0(3)$ \\
\hline $\mathrm{P}(11)$ & $\operatorname{Pt}(1)$ & $C(1)$ & $93.5(7)$ & $\operatorname{Pt}(2)$ & $\operatorname{Pt}(3)$ & $C(2)$ & $176.5(8)$ \\
\hline $\mathrm{P}(21)$ & $\operatorname{Pt}(1)$ & $\mathrm{P}(31)$ & $147.9(4)$ & $\mathrm{P}(13)$ & $\operatorname{Pt}(3)$ & $\mathrm{P}(23)$ & $33.2(4)$ \\
\hline$P(21)$ & $\operatorname{Pt}(1)$ & $\mathrm{P}(41)$ & $176.2(4)$ & $P(13)$ & $\operatorname{Pt}(3)$ & $P(33)$ & $177.2(4)$ \\
\hline$P(21)$ & $\operatorname{Pt}(1)$ & $C(1)$ & $92.8(8)$ & $\mathrm{P}(13)$ & $\operatorname{Pt}(3)$ & $\mathrm{P}(43)$ & $146.7(5)$ \\
\hline $\mathrm{P}(31)$ & $\operatorname{Pt}(1)$ & $\mathrm{P}(41)$ & $34.5(4)$ & $\mathrm{P}(13)$ & $\operatorname{Pt}(3)$ & $C(2)$ & $92.4(6)$ \\
\hline $\mathrm{P}(31)$ & $\operatorname{Pt}(1)$ & $C(1)$ & $95.2(7)$ & $\mathrm{P}(23)$ & $\operatorname{Pt}(3)$ & $\mathrm{P}(33)$ & $144.4(5)$ \\
\hline $\mathrm{P}(41)$ & $\operatorname{Pt}(1)$ & $C(1)$ & $89.6(8)$ & $P(23)$ & $\operatorname{Pt}(3)$ & $P(43)$ & $170.5(4)$ \\
\hline $\operatorname{Pt}(1)$ & $\operatorname{Pt}(2)$ & $\operatorname{Pt}(3)$ & $179.48(5)$ & $P(23)$ & $\operatorname{Pt}(3)$ & $C(2)$ & $93.5(7)$ \\
\hline $\operatorname{Pt}(1)$ & $\operatorname{Pt}(2)$ & $\mathrm{P}(12)$ & $90.7(2)$ & $\mathrm{P}(33)$ & $\operatorname{Pt}(3)$ & $\mathrm{P}(43)$ & $35.1(5)$ \\
\hline $\operatorname{Pt}(1)$ & $\operatorname{Pt}(2)$ & $\mathrm{P}(22)$ & $89.1(3)$ & $\mathrm{P}(33)$ & $\operatorname{Pt}(3)$ & $C(2)$ & $89.2(6)$ \\
\hline $\operatorname{Pt}(1)$ & $\operatorname{Pt}(2)$ & $\mathrm{P}(32)$ & $92.4(2)$ & $\mathrm{P}(43)$ & $\operatorname{Pt}(3)$ & $C(2)$ & $95.9(7)$ \\
\hline $\operatorname{Pt}(1)$ & $\operatorname{Pt}(2)$ & $\mathrm{P}(42)$ & $87.2(3)$ & $\operatorname{Pt}(1)$ & $\mathrm{P}(11)$ & $\mathrm{P}(21)$ & $79.5(5)$ \\
\hline $\operatorname{Pt}(3)$ & $\operatorname{Pt}(2)$ & $\mathrm{P}(12)$ & $89.1(2)$ & $\operatorname{Pt}(1)$ & $\mathrm{P}(11)$ & $\mathrm{P}(22)$ & $96.7(3)$ \\
\hline $\operatorname{Pt}(3)$ & $\operatorname{Pt}(2)$ & $P(22)$ & $91.1(3)$ & $\operatorname{Pt}(1)$ & $\mathrm{P}(11)$ & $C(3)$ & $110.0(8)$ \\
\hline $\operatorname{Pt}(3)$ & $\operatorname{Pt}(2)$ & $P(32)$ & $87.8(2)$ & $\operatorname{Pt}(1)$ & $P(11)$ & $C(75)$ & $115(1)$ \\
\hline $\operatorname{Pt}(3)$ & $\operatorname{Pt}(2)$ & $\mathrm{P}(42)$ & $92.6(3)$ & $\operatorname{Pt}(1)$ & $\mathrm{P}(11)$ & $C(21)$ & $116.2(6)$ \\
\hline $\mathrm{P}(12)$ & $\operatorname{Pt}(2)$ & $\mathrm{P}(22)$ & $37.9(4)$ & $\mathrm{P}(21)$ & $P(11)$ & $\mathrm{P}(22)$ & $95.3(7)$ \\
\hline$P(12)$ & $\operatorname{Pt}(2)$ & $\mathrm{P}(32)$ & $176.9(3)$ & $\mathrm{P}(21)$ & $P(11)$ & $C(3)$ & $50(1)$ \\
\hline $\mathrm{P}(21)$ & $\mathrm{P}(11)$ & $C(75)$ & 153(1) & $C(4)$ & $\mathrm{P}(12)$ & $C(93)$ & $82(1)$ \\
\hline $\mathrm{P}(21)$ & $\mathrm{P}(11)$ & $C(21)$ & $84.4(9)$ & $C(4)$ & $P(12)$ & $C(99)$ & $108(1)$ \\
\hline $\mathrm{P}(22)$ & $\mathrm{P}(11)$ & $C(3)$ & $53.8(9)$ & $C(93)$ & $\mathrm{P}(12)$ & $C(99)$ & $30(1)$ \\
\hline $\mathrm{P}(22)$ & $\mathrm{P}(11)$ & $C(75)$ & $62(1)$ & $\operatorname{Pt}(3)$ & $P(13)$ & $\mathrm{P}(23)$ & $70.6(6)$ \\
\hline $\mathrm{P}(22)$ & $\mathrm{P}(11)$ & $C(21)$ & $146.3(6)$ & $\operatorname{Pt}(3)$ & $P(13)$ & $C(4)$ & $121.0(8)$ \\
\hline$C(3)$ & $\mathrm{P}(11)$ & $C(75)$ & 103(1) & $\operatorname{Pt}(3)$ & $P(13)$ & $C(51)$ & $113.2(7)$ \\
\hline$C(3)$ & $\mathrm{P}(11)$ & $C(21)$ & $105(1)$ & $\operatorname{Pt}(3)$ & $P(13)$ & $C(63)$ & $109.3(6)$ \\
\hline$C(75)$ & $\mathrm{P}(11)$ & $C(21)$ & 107(1) & $\mathrm{P}(23)$ & $\mathrm{P}(13)$ & $C(4)$ & $76(1)$ \\
\hline $\operatorname{Pt}(2)$ & $\mathrm{P}(12)$ & $\mathrm{P}(22)$ & $72.1(5)$ & $P(23)$ & $P(13)$ & $C(51)$ & 174(1) \\
\hline $\operatorname{Pt}(2)$ & $\mathrm{P}(12)$ & $\mathrm{P}(23)$ & $90.0(4)$ & $P(23)$ & $\mathrm{P}(13)$ & $C(63)$ & $70.3(9)$ \\
\hline $\operatorname{Pt}(2)$ & $\mathrm{P}(12)$ & $C(3)$ & $115(1)$ & $C(4)$ & $\mathrm{P}(13)$ & $C(51)$ & $105(1)$ \\
\hline $\operatorname{Pt}(2)$ & $P(12)$ & $C(4)$ & $109.5(9)$ & $C(4)$ & $\mathrm{P}(13)$ & $C(63)$ & 103(1) \\
\hline $\operatorname{Pt}(2)$ & $\mathrm{P}(12)$ & $C(93)$ & 111(1) & $C(51)$ & $\mathrm{P}(13)$ & $C(63)$ & $103.7(9)$ \\
\hline $\operatorname{Pt}(2)$ & $P(12)$ & $C(99)$ & $113.7(9)$ & $\operatorname{Pt}(1)$ & $\mathrm{P}(21)$ & $\mathrm{P}(11)$ & $65.8(6)$ \\
\hline$P(22)$ & $\mathrm{P}(12)$ & $P(23)$ & $89.9(6)$ & $\operatorname{Pt}(1)$ & $P(21)$ & $C(3)$ & 119(1) \\
\hline $\mathrm{P}(22)$ & $\mathrm{P}(12)$ & $C(3)$ & $78(1)$ & $\operatorname{Pt}(1)$ & $P(21)$ & $C(21)$ & $97.2(6)$ \\
\hline $\mathrm{P}(22)$ & $\mathrm{P}(12)$ & $C(4)$ & $57.9(9)$ & $\operatorname{Pt}(1)$ & $\mathrm{P}(21)$ & $C(27)$ & $116.4(7)$ \\
\hline $\mathrm{P}(22)$ & $P(12)$ & $C(93)$ & $136(1)$ & $\mathrm{P}(11)$ & $\mathrm{P}(21)$ & $C(3)$ & $82(1)$ \\
\hline $\mathrm{P}(22)$ & $\mathrm{P}(12)$ & $C(99)$ & $165(1)$ & $P(11)$ & $\mathrm{P}(21)$ & $C(21)$ & $55.9(7)$ \\
\hline $\mathrm{P}(23)$ & $\mathrm{P}(12)$ & $C(3)$ & $146.5(8)$ & $\mathrm{P}(11)$ & $\mathrm{P}(21)$ & $C(27)$ & $173(1)$ \\
\hline$P(23)$ & $\mathrm{P}(12)$ & $C(4)$ & $48.1(7)$ & $C(3)$ & $P(21)$ & $C(21)$ & 105(1) \\
\hline $\mathrm{P}(23)$ & $\mathrm{P}(12)$ & $C(93)$ & $47(1)$ & $C(3)$ & $\mathrm{P}(21)$ & $C(27)$ & 102(2) \\
\hline$P(23)$ & $\mathrm{P}(12)$ & $C(99)$ & $77(1)$ & $C(21)$ & $P(21)$ & $C(27)$ & $117.5(9)$ \\
\hline$C(3)$ & $\mathrm{P}(12)$ & $C(4)$ & $100(1)$ & $\operatorname{Pt}(2)$ & $\mathrm{P}(22)$ & $\mathrm{P}(11)$ & $88.8(4)$ \\
\hline$C(3)$ & $\mathrm{P}(12)$ & $C(93)$ & $130(2)$ & $\operatorname{Pt}(2)$ & $P(22)$ & $P(12)$ & $70.0(5)$ \\
\hline
\end{tabular}




\begin{tabular}{|c|c|c|c|c|c|c|c|}
\hline$C(3)$ & $P(12)$ & $C(99)$ & $109(1)$ & $\operatorname{Pt}(2)$ & $P(22)$ & $C(3)$ & $102.6(7)$ \\
\hline $\operatorname{Pt}(2)$ & $\mathrm{P}(22)$ & $C(4)$ & $118.4(9)$ & $\mathrm{P}(13)$ & $\mathrm{P}(23)$ & $C(93)$ & $154(1)$ \\
\hline $\operatorname{Pt}(2)$ & $\mathrm{P}(22)$ & $C(81)$ & $112(1)$ & $\mathrm{P}(13)$ & $\mathrm{P}(23)$ & $C(69)$ & $87(1)$ \\
\hline $\mathrm{P}(11)$ & $\mathrm{P}(22)$ & $\mathrm{P}(12)$ & $90.9(6)$ & $\mathrm{P}(13)$ & $\mathrm{P}(23)$ & $C(63)$ & $68.6(9)$ \\
\hline $\mathrm{P}(11)$ & $\mathrm{P}(22)$ & $C(3)$ & $46.5(8)$ & $\mathrm{P}(13)$ & $P(23)$ & $C(68)$ & $109.0(9)$ \\
\hline $\mathrm{P}(11)$ & $\mathrm{P}(22)$ & $C(4)$ & $141(1)$ & $C(4)$ & $\mathrm{P}(23)$ & $C(93)$ & $92(2)$ \\
\hline $\mathrm{P}(11)$ & $P(22)$ & $C(81)$ & $75(1)$ & $C(4)$ & $P(23)$ & $C(69)$ & $108(1)$ \\
\hline $\mathrm{P}(12)$ & $\mathrm{P}(22)$ & $C(3)$ & $56.2(9)$ & $C(4)$ & $\mathrm{P}(23)$ & $C(63)$ & $96(1)$ \\
\hline $\mathrm{P}(12)$ & $\mathrm{P}(22)$ & $C(4)$ & $75(1)$ & $C(4)$ & $\mathrm{P}(23)$ & $C(68)$ & $116(1)$ \\
\hline $\mathrm{P}(12)$ & $P(22)$ & $C(81)$ & $166(2)$ & $C(93)$ & $P(23)$ & $C(69)$ & $108(2)$ \\
\hline$C(3)$ & $\mathrm{P}(22)$ & $C(4)$ & $98(1)$ & $C(93)$ & $\mathrm{P}(23)$ & $C(63)$ & $124(2)$ \\
\hline$C(3)$ & $P(22)$ & $C(81)$ & $111(2)$ & $C(93)$ & $P(23)$ & $C(68)$ & $86(2)$ \\
\hline$C(4)$ & $P(22)$ & $C(81)$ & $114(2)$ & $C(69)$ & $P(23)$ & $C(63)$ & 19(1) \\
\hline $\operatorname{Pt}(3)$ & $P(23)$ & $\mathrm{P}(12)$ & $95.2(5)$ & $C(69)$ & $\mathrm{P}(23)$ & $C(68)$ & $22(1)$ \\
\hline $\mathrm{Pt}(3)$ & $P(23)$ & $P(13)$ & $76.3(8)$ & $C(63)$ & $P(23)$ & $C(68)$ & $40(1)$ \\
\hline $\operatorname{Pt}(3)$ & $P(23)$ & $C(4)$ & $117(1)$ & $\operatorname{Pt}(1)$ & $\mathrm{P}(31)$ & $\mathrm{P}(41)$ & $68.5(6)$ \\
\hline $\operatorname{Pt}(3)$ & $P(23)$ & $C(93)$ & $114(1)$ & $\operatorname{Pt}(1)$ & $\mathrm{P}(31)$ & $\mathrm{P}(42)$ & $91.5(4)$ \\
\hline $\mathrm{Pt}(3)$ & $P(23)$ & $C(69)$ & $115.1(8)$ & $\operatorname{Pt}(1)$ & $P(31)$ & $C(5)$ & $112.0(7)$ \\
\hline $\operatorname{Pt}(3)$ & $P(23)$ & $C(63)$ & 111.3(8) & $\operatorname{Pt}(1)$ & $\mathrm{P}(31)$ & $C(102)$ & $117(1)$ \\
\hline $\mathrm{Pt}(3)$ & $\mathrm{P}(23)$ & $C(68)$ & $122.0(7)$ & $\operatorname{Pt}(1)$ & $\mathrm{P}(31)$ & $C(33)$ & $112.6(7)$ \\
\hline $\mathrm{P}(12)$ & $P(23)$ & $\mathrm{P}(13)$ & 98.4(7) & $\mathrm{P}(41)$ & $\mathrm{P}(31)$ & $\mathrm{P}(42)$ & 98.2(7) \\
\hline $\mathrm{P}(12)$ & $P(23)$ & $C(4)$ & $51.3(7)$ & $\mathrm{P}(41)$ & $\mathrm{P}(31)$ & $C(5)$ & $63.0(9)$ \\
\hline $\mathrm{P}(12)$ & $P(23)$ & $C(93)$ & $58(1)$ & $\mathrm{P}(41)$ & $\mathrm{P}(31)$ & $C(102)$ & $164(1)$ \\
\hline $\mathrm{P}(12)$ & $P(23)$ & $C(69)$ & $149.6(9)$ & $\mathrm{P}(41)$ & $\mathrm{P}(31)$ & $C(33)$ & $77(1)$ \\
\hline $\mathrm{P}(12)$ & $P(23)$ & $C(63)$ & $145.5(8)$ & $\mathrm{P}(42)$ & $\mathrm{P}(31)$ & $C(5)$ & $54.1(8)$ \\
\hline $\mathrm{P}(12)$ & $P(23)$ & $C(68)$ & 138(1) & $\mathrm{P}(42)$ & $\mathrm{P}(31)$ & $C(102)$ & $67(1)$ \\
\hline $\mathrm{P}(13)$ & $\mathrm{P}(23)$ & $\mathrm{C}(4)$ & $62(1)$ & $\mathrm{P}(42)$ & $\mathrm{P}(31)$ & $C(33)$ & $151.0(8)$ \\
\hline$C(5)$ & $\mathrm{P}(31)$ & $C(102)$ & $102(1)$ & $\mathrm{P}(43)$ & $\mathrm{P}(33)$ & $C(112)$ & $64(3)$ \\
\hline$C(5)$ & $\mathrm{P}(31)$ & $C(33)$ & $100(1)$ & $C(6)$ & $\mathrm{P}(33)$ & $C(45)$ & $103(1)$ \\
\hline$C(102)$ & $\mathrm{P}(31)$ & $C(33)$ & $112(1)$ & $C(6)$ & $\mathrm{P}(33)$ & $C(57)$ & 109(1) \\
\hline $\operatorname{Pt}(2)$ & $P(32)$ & $\mathrm{P}(42)$ & $69.7(6)$ & $C(6)$ & $\mathrm{P}(33)$ & $C(112)$ & $108(2)$ \\
\hline $\mathrm{Pt}(2)$ & $\mathrm{P}(32)$ & $\mathrm{P}(43)$ & $92.7(4)$ & $C(45)$ & $\mathrm{P}(33)$ & $C(57)$ & $104(1)$ \\
\hline $\operatorname{Pt}(2)$ & $P(32)$ & $C(5)$ & $118.2(8)$ & $C(45)$ & $P(33)$ & $C(112)$ & $112(3)$ \\
\hline $\operatorname{Pt}(2)$ & $P(32)$ & $C(6)$ & 105.1(8) & $C(57)$ & $\mathrm{P}(33)$ & $C(112)$ & $8(3)$ \\
\hline $\mathrm{Pt}(2)$ & $\mathrm{P}(32)$ & $C(85)$ & $120(1)$ & $\operatorname{Pt}(1)$ & $\mathrm{P}(41)$ & $\mathrm{P}(31)$ & $77.0(7)$ \\
\hline $\mathrm{P}(42)$ & $\mathrm{P}(32)$ & $P(43)$ & $95.0(7)$ & $\operatorname{Pt}(1)$ & $\mathrm{P}(41)$ & $C(5)$ & $123(1)$ \\
\hline $\mathrm{P}(42)$ & $P(32)$ & $C(5)$ & $78(1)$ & $\operatorname{Pt}(1)$ & $\mathrm{P}(41)$ & $C(33)$ & $110.1(8)$ \\
\hline $\mathrm{P}(42)$ & $\mathrm{P}(32)$ & $C(6)$ & $58.2(9)$ & $\operatorname{Pt}(1)$ & $\mathrm{P}(41)$ & $C(39)$ & $115.1(9)$ \\
\hline $\mathrm{P}(42)$ & $P(32)$ & $C(85)$ & $165(1)$ & $\mathrm{P}(31)$ & $P(41)$ & $C(5)$ & $73(1)$ \\
\hline $\mathrm{P}(43)$ & $\mathrm{P}(32)$ & $C(5)$ & 142.1(8) & $\mathrm{P}(31)$ & $\mathrm{P}(41)$ & $C(33)$ & $63.2(9)$ \\
\hline $\mathrm{P}(43)$ & $P(32)$ & $C(6)$ & $48.2(7)$ & $\mathrm{P}(31)$ & $\mathrm{P}(41)$ & $C(39)$ & $165(1)$ \\
\hline $\mathrm{P}(43)$ & $P(32)$ & $C(85)$ & $74(1)$ & $C(5)$ & $P(41)$ & $C(33)$ & $98(1)$ \\
\hline$C(5)$ & $\mathrm{P}(32)$ & $C(6)$ & $99.6(9)$ & $C(5)$ & $\mathrm{P}(41)$ & $C(39)$ & 104(1) \\
\hline$C(5)$ & $\mathrm{P}(32)$ & $C(85)$ & $104(1)$ & $C(33)$ & $\mathrm{P}(41)$ & $C(39)$ & $104(1)$ \\
\hline$C(6)$ & $P(32)$ & $C(85)$ & 107(1) & $\operatorname{Pt}(2)$ & $P(42)$ & $\mathrm{P}(31)$ & $95.2(4)$ \\
\hline $\mathrm{Pt}(3)$ & $\mathrm{P}(33)$ & $P(43)$ & $72.6(6)$ & $\operatorname{Pt}(2)$ & $P(42)$ & $\mathrm{P}(32)$ & $73.4(5)$ \\
\hline $\operatorname{Pt}(3)$ & $P(33)$ & $C(6)$ & $118.5(8)$ & $\mathrm{Pt}(2)$ & $\mathrm{P}(42)$ & $C(5)$ & $110.2(7)$ \\
\hline $\mathrm{Pt}(3)$ & $\mathrm{P}(33)$ & $C(45)$ & $114.0(8)$ & $\operatorname{Pt}(2)$ & $\mathrm{P}(42)$ & $C(6)$ & $117.4(8)$ \\
\hline $\operatorname{Pt}(3)$ & $\mathrm{P}(33)$ & $C(57)$ & 108.1(7) & $\operatorname{Pt}(2)$ & $\mathrm{P}(42)$ & $C(108)$ & $117(2)$ \\
\hline $\operatorname{Pt}(3)$ & $P(33)$ & $C(112)$ & $102(2)$ & $\mathrm{P}(31)$ & $\mathrm{P}(42)$ & $\mathrm{P}(32)$ & $95.8(7)$ \\
\hline $\mathrm{P}(43)$ & $\mathrm{P}(33)$ & $C(6)$ & $75(1)$ & $\mathrm{P}(31)$ & $\mathrm{P}(42)$ & $C(5)$ & $48.1(7)$ \\
\hline $\mathrm{P}(43)$ & $P(33)$ & $C(45)$ & $173(1)$ & $\mathrm{P}(31)$ & $\mathrm{P}(42)$ & $C(6)$ & $142(1)$ \\
\hline $\mathrm{P}(43)$ & $\mathrm{P}(33)$ & C(57) & $71(1)$ & $\mathrm{P}(31)$ & $\mathrm{P}(42)$ & C(108) & $66(2)$ \\
\hline $\mathrm{P}(32)$ & $\mathrm{P}(42)$ & $C(5)$ & $59.2(9)$ & $C(6)$ & $\mathrm{P}(43)$ & $C(58)$ & $124(1)$ \\
\hline $\mathrm{P}(32)$ & $\mathrm{P}(42)$ & $C(6)$ & $78(1)$ & $C(6)$ & $\mathrm{P}(43)$ & $C(112)$ & $107(3)$ \\
\hline
\end{tabular}




\begin{tabular}{|c|c|c|c|c|c|c|c|}
\hline $\mathrm{P}(32)$ & $\mathrm{P}(42)$ & $C(108)$ & $159(2)$ & $C(91)$ & $\mathrm{P}(43)$ & $C(57)$ & $123(2)$ \\
\hline$C(5)$ & $\mathrm{P}(42)$ & $C(6)$ & $100(1)$ & $\mathrm{C}(91)$ & $\mathrm{P}(43)$ & C(58) & $87(2)$ \\
\hline$C(5)$ & $\mathrm{P}(42)$ & $C(108)$ & $100(2)$ & $C(91)$ & $\mathrm{P}(43)$ & $C(112)$ & $116(3)$ \\
\hline$C(6)$ & $\mathrm{P}(42)$ & $C(108)$ & $109(2)$ & $C(57)$ & $\mathrm{P}(43)$ & $C(58)$ & $39(1)$ \\
\hline $\operatorname{Pt}(3)$ & $\mathrm{P}(43)$ & $\mathrm{P}(32)$ & $94.3(5)$ & $C(57)$ & $\mathrm{P}(43)$ & $C(112)$ & 10(3) \\
\hline $\operatorname{Pt}(3)$ & $\mathrm{P}(43)$ & $\mathrm{P}(33)$ & $72.3(7)$ & $C(58)$ & $\mathrm{P}(43)$ & $C(112)$ & $30(3)$ \\
\hline $\operatorname{Pt}(3)$ & $\mathrm{P}(43)$ & $C(6)$ & $110(1)$ & $\mathrm{F}(11)$ & $\mathrm{P}(51)$ & $F(12)$ & $89(1)$ \\
\hline $\mathrm{Pt}(3)$ & $\mathrm{P}(43)$ & $C(91)$ & $118(1)$ & $\mathrm{F}(11)$ & $\mathrm{P}(51)$ & $F(13)$ & $175(1)$ \\
\hline $\operatorname{Pt}(3)$ & $\mathrm{P}(43)$ & C(57) & $105.9(8)$ & $\mathrm{F}(11)$ & $P(51)$ & $\mathrm{F}(14)$ & $88(1)$ \\
\hline $\operatorname{Pt}(3)$ & $\mathrm{P}(43)$ & $C(58)$ & $116.0(8)$ & $\mathrm{F}(11)$ & $P(51)$ & $F(15)$ & $86(1)$ \\
\hline $\operatorname{Pt}(3)$ & $\mathrm{P}(43)$ & $C(112)$ & 107(2) & $\mathrm{F}(11)$ & $\mathrm{P}(51)$ & $F(16)$ & $88(1)$ \\
\hline $\mathrm{P}(32)$ & $\mathrm{P}(43)$ & $\mathrm{P}(33)$ & $100.3(8)$ & $F(12)$ & $\mathrm{P}(51)$ & $F(13)$ & $90(1)$ \\
\hline $\mathrm{P}(32)$ & $\mathrm{P}(43)$ & $C(6)$ & $53.8(7)$ & $\mathrm{F}(12)$ & $P(51)$ & $\mathrm{F}(14)$ & $94(1)$ \\
\hline $\mathrm{P}(32)$ & $\mathrm{P}(43)$ & $C(91)$ & $61(2)$ & $\mathrm{F}(12)$ & $P(51)$ & $F(15)$ & $86(1)$ \\
\hline $\mathrm{P}(32)$ & $\mathrm{P}(43)$ & C(57) & $150.4(9)$ & $F(12)$ & $\mathrm{P}(51)$ & $F(16)$ & $175.4(9)$ \\
\hline $\mathrm{P}(32)$ & $\mathrm{P}(43)$ & $C(58)$ & $145(1)$ & $F(13)$ & $P(51)$ & $F(14)$ & $97(1)$ \\
\hline $\mathrm{P}(32)$ & $\mathrm{P}(43)$ & $C(112)$ & $156(2)$ & $F(13)$ & $P(51)$ & $F(15)$ & $89(1)$ \\
\hline $\mathrm{P}(33)$ & $\mathrm{P}(43)$ & $C(6)$ & $59(1)$ & $\mathrm{F}(13)$ & $\mathrm{P}(51)$ & $F(16)$ & 93(1) \\
\hline $\mathrm{P}(33)$ & $\mathrm{P}(43)$ & $C(91)$ & $158(2)$ & $\mathrm{F}(14)$ & $P(51)$ & $F(15)$ & $174(1)$ \\
\hline $\mathrm{P}(33)$ & $\mathrm{P}(43)$ & $C(57)$ & $67(1)$ & $\mathrm{F}(14)$ & $P(51)$ & $F(16)$ & $89(1)$ \\
\hline $\mathrm{P}(33)$ & $\mathrm{P}(43)$ & $C(58)$ & $106(1)$ & $\mathrm{F}(15)$ & $P(51)$ & $F(16)$ & $91(1)$ \\
\hline $\mathrm{P}(33)$ & $\mathrm{P}(43)$ & $C(112)$ & $76(3)$ & $\mathrm{F}(21)$ & $P(52)$ & $F(22)$ & $90(1)$ \\
\hline$C(6)$ & $\mathrm{P}(43)$ & $C(91)$ & $99(2)$ & $\mathrm{F}(21)$ & $P(52)$ & $F(23)$ & $87(2)$ \\
\hline$C(6)$ & $\mathrm{P}(43)$ & $C(57)$ & $98(1)$ & $\mathrm{F}(21)$ & $P(52)$ & $F(24)$ & $97(2)$ \\
\hline $\mathrm{F}(21)$ & $\mathrm{P}(52)$ & $\mathrm{F}(25)$ & 94(1) & $\mathrm{P}(13)$ & $C(4)$ & $P(23)$ & $42.2(7)$ \\
\hline $\mathrm{F}(21)$ & $P(52)$ & $F(26)$ & $164(2)$ & $\mathrm{P}(22)$ & $C(4)$ & $\mathrm{P}(23)$ & $107(1)$ \\
\hline $\mathrm{F}(22)$ & $P(52)$ & $F(23)$ & $78(1)$ & $\mathrm{P}(31)$ & $C(5)$ & $\mathrm{P}(32)$ & $108(1)$ \\
\hline $\mathrm{F}(22)$ & $\mathrm{P}(52)$ & $\mathrm{F}(24)$ & $89(2)$ & $\mathrm{P}(31)$ & $C(5)$ & $\mathrm{P}(41)$ & $44.1(8)$ \\
\hline $\mathrm{F}(22)$ & $P(52)$ & $F(25)$ & 176(1) & $\mathrm{P}(31)$ & $C(5)$ & $\mathrm{P}(42)$ & $78(1)$ \\
\hline $\mathrm{F}(22)$ & $\mathrm{P}(52)$ & $\mathrm{F}(26)$ & $89(1)$ & $\mathrm{P}(32)$ & $C(5)$ & $\mathrm{P}(41)$ & $105(1)$ \\
\hline $\mathrm{F}(23)$ & $P(52)$ & $\mathrm{F}(24)$ & $167(2)$ & $\mathrm{P}(32)$ & $C(5)$ & $\mathrm{P}(42)$ & $43.1(7)$ \\
\hline $\mathrm{F}(23)$ & $\mathrm{P}(52)$ & $\mathrm{F}(25)$ & $103(2)$ & $\mathrm{P}(41)$ & $C(5)$ & $\mathrm{P}(42)$ & 103(1) \\
\hline$F(23)$ & $P(52)$ & $F(26)$ & $77(2)$ & $P(32)$ & $C(6)$ & $\mathrm{P}(33)$ & $110(1)$ \\
\hline $\mathrm{F}(24)$ & $P(52)$ & $F(25)$ & $90(2)$ & $\mathrm{P}(32)$ & $C(6)$ & $\mathrm{P}(42)$ & $43.9(7)$ \\
\hline $\mathrm{F}(24)$ & $\mathrm{P}(52)$ & $\mathrm{F}(26)$ & $99(2)$ & $\mathrm{P}(32)$ & $C(6)$ & $\mathrm{P}(43)$ & $78.0(9)$ \\
\hline $\mathrm{F}(25)$ & $\mathrm{P}(52)$ & $\mathrm{F}(26)$ & $87(1)$ & $\mathrm{P}(33)$ & $C(6)$ & $\mathrm{P}(42)$ & 112(1) \\
\hline$C(1)$ & $\mathrm{N}(1)$ & $C(11)$ & $177(3)$ & $\mathrm{P}(33)$ & $C(6)$ & $\mathrm{P}(43)$ & $46.3(8)$ \\
\hline$C(2)$ & $\mathrm{N}(2)$ & $C(14)$ & $178(2)$ & $\mathrm{P}(42)$ & $C(6)$ & $\mathrm{P}(43)$ & $109(1)$ \\
\hline $\operatorname{Pt}(1)$ & $C(1)$ & $\mathrm{N}(1)$ & $173(3)$ & $\mathrm{N}(1)$ & $C(11)$ & $C(12)$ & $112(2)$ \\
\hline $\operatorname{Pt}(3)$ & $C(2)$ & $N(2)$ & $178(3)$ & $\mathrm{N}(1)$ & $C(11)$ & $C(16)$ & $120(2)$ \\
\hline $\mathrm{P}(11)$ & $C(3)$ & $\mathrm{P}(12)$ & $110(1)$ & $C(12)$ & $C(11)$ & $C(16)$ & $127(2)$ \\
\hline $\mathrm{P}(11)$ & $C(3)$ & $\mathrm{P}(21)$ & $48(1)$ & $C(11)$ & $C(12)$ & $C(13)$ & $113(2)$ \\
\hline $\mathrm{P}(11)$ & $C(3)$ & $\mathrm{P}(22)$ & $80(1)$ & $C(11)$ & $C(12)$ & $C(17)$ & $125(2)$ \\
\hline $\mathrm{P}(12)$ & $C(3)$ & $\mathrm{P}(21)$ & 114(1) & $C(13)$ & $C(12)$ & $C(17)$ & $121(2)$ \\
\hline $\mathrm{P}(12)$ & $C(3)$ & $\mathrm{P}(22)$ & $45.4(8)$ & $C(12)$ & $C(13)$ & $C(14)$ & $119(2)$ \\
\hline $\mathrm{P}(21)$ & $C(3)$ & $\mathrm{P}(22)$ & $116(2)$ & $C(12)$ & $C(13)$ & $C(18)$ & $118(2)$ \\
\hline $\mathrm{P}(12)$ & $C(4)$ & $\mathrm{P}(13)$ & $108(1)$ & $C(14)$ & $C(13)$ & C(18) & $122(2)$ \\
\hline $\mathrm{P}(12)$ & $C(4)$ & $\mathrm{P}(22)$ & $46.8(7)$ & $\mathrm{N}(2)$ & $C(14)$ & $C(13)$ & $120(2)$ \\
\hline $\mathrm{P}(12)$ & $C(4)$ & $\mathrm{P}(23)$ & $80.6(9)$ & $\mathrm{N}(2)$ & $C(14)$ & $C(15)$ & $113(2)$ \\
\hline $\mathrm{P}(13)$ & $C(4)$ & $\mathrm{P}(22)$ & $103(1)$ & $C(13)$ & $C(14)$ & $C(15)$ & $127(2)$ \\
\hline$C(14)$ & $C(15)$ & $C(16)$ & $116(2)$ & $\mathrm{C}(79)$ & $C(78)$ & $C(83)$ & $77(5)$ \\
\hline$C(14)$ & $C(15)$ & C(19) & $126(2)$ & $\mathrm{C}(78)$ & $C(79)$ & $C(80)$ & $122(3)$ \\
\hline$C(16)$ & $C(15)$ & $\mathrm{C}(19)$ & $118(2)$ & $\mathrm{C}(78)$ & $C(79)$ & $C(82)$ & $113(4)$ \\
\hline$C(11)$ & $C(16)$ & $C(15)$ & $116(2)$ & $C(78)$ & $C(79)$ & $C(83)$ & $48(4)$ \\
\hline$C(11)$ & $C(16)$ & $C(20)$ & $120(2)$ & $C(80)$ & $C(79)$ & $C(82)$ & $45(3)$ \\
\hline$C(15)$ & $C(16)$ & $C(20)$ & $123(2)$ & $C(80)$ & $C(79)$ & $C(83)$ & $109(3)$ \\
\hline
\end{tabular}




\begin{tabular}{|c|c|c|c|c|c|c|c|}
\hline$P(11)$ & $C(75)$ & $C(76)$ & $126(2)$ & $\mathrm{C}(82)$ & $C(79)$ & $C(83)$ & $140(4)$ \\
\hline $\mathrm{P}(11)$ & $C(75)$ & $C(80)$ & 112(3) & $C(75)$ & $C(80)$ & $C(79)$ & 112(3) \\
\hline $\mathrm{P}(11)$ & $C(75)$ & $C(81)$ & $126(4)$ & $C(75)$ & $C(80)$ & $C(81)$ & $38(2)$ \\
\hline $\mathrm{C}(76)$ & $C(75)$ & C(80) & $123(3)$ & $\mathrm{C}(75)$ & $C(80)$ & $C(82)$ & $111(4)$ \\
\hline$C(76)$ & $C(75)$ & $C(81)$ & $61(3)$ & $C(79)$ & $C(80)$ & $C(81)$ & $90(3)$ \\
\hline$C(80)$ & $C(75)$ & $C(81)$ & $85(3)$ & $C(79)$ & $C(80)$ & $C(82)$ & $57(4)$ \\
\hline$C(75)$ & $C(76)$ & C(77) & $117(3)$ & $\mathrm{C}(81)$ & $C(80)$ & $C(82)$ & $72(3)$ \\
\hline$C(75)$ & $C(76)$ & $C(81)$ & $51(3)$ & $\mathrm{P}(22)$ & $C(81)$ & $C(75)$ & $97(4)$ \\
\hline$C(75)$ & $C(76)$ & $C(84)$ & $114(3)$ & $\mathrm{P}(22)$ & $C(81)$ & $C(76)$ & $123(3)$ \\
\hline C(77) & $C(76)$ & $C(81)$ & $89(3)$ & $\mathrm{P}(22)$ & $C(81)$ & C(77) & $154(3)$ \\
\hline C(77) & $C(76)$ & $C(84)$ & $45(4)$ & $\mathrm{P}(22)$ & $C(81)$ & $C(80)$ & $107(3)$ \\
\hline$C(81)$ & $C(76)$ & $C(84)$ & $123(3)$ & $\mathrm{P}(22)$ & $C(81)$ & $C(82)$ & $104(3)$ \\
\hline$C(76)$ & $C(77)$ & $C(78)$ & $131(5)$ & $C(75)$ & $C(81)$ & $C(76)$ & $69(3)$ \\
\hline C(76) & C(77) & $C(81)$ & $45(3)$ & $C(75)$ & $C(81)$ & $C(77)$ & $98(4)$ \\
\hline$C(76)$ & $C(77)$ & $C(84)$ & $72(5)$ & $C(75)$ & $C(81)$ & $C(80)$ & $56(2)$ \\
\hline C(78) & $C(77)$ & $C(81)$ & $96(4)$ & $C(75)$ & $C(81)$ & $C(82)$ & $91(3)$ \\
\hline$C(78)$ & C(77) & $C(84)$ & $105(5)$ & $C(76)$ & C(81) & $\mathrm{C}(77)$ & $46(2)$ \\
\hline$C(81)$ & $C(77)$ & $C(84)$ & $109(6)$ & $C(76)$ & $C(81)$ & $C(80)$ & $108(3)$ \\
\hline$C(77)$ & $C(78)$ & $C(79)$ & $115(5)$ & $C(76)$ & $C(81)$ & $C(82)$ & $130(4)$ \\
\hline$C(77)$ & $C(78)$ & $C(83)$ & $88(5)$ & $C(77)$ & $C(81)$ & $C(80)$ & 100(3) \\
\hline$C(77)$ & $C(81)$ & $C(82)$ & $97(3)$ & $C(91)$ & $C(86)$ & $C(92)$ & $59(4)$ \\
\hline$C(80)$ & $C(81)$ & $C(82)$ & $35(2)$ & $C(86)$ & $C(87)$ & $C(88)$ & $128(4)$ \\
\hline$C(79)$ & $C(82)$ & $C(80)$ & $77(5)$ & $C(86)$ & $C(87)$ & $C(92)$ & $39(3)$ \\
\hline $\mathrm{C}(79)$ & $\mathrm{C}(82)$ & $\mathrm{C}(81)$ & $97(4)$ & $\mathrm{C}(88)$ & C (87) & $C(92)$ & $126(4)$ \\
\hline $\mathrm{C}(80)$ & $C(82)$ & C(81) & $73(3)$ & $\mathrm{C}(87)$ & C(88) & $C(89)$ & $113(4)$ \\
\hline C(78) & $C(83)$ & $C(79)$ & $55(4)$ & $C(88)$ & C(89) & $C(90)$ & $123(3)$ \\
\hline$C(78)$ & $C(83)$ & $C(84)$ & $82(5)$ & $C(85)$ & $C(90)$ & C(89) & $110(3)$ \\
\hline$C(79)$ & $C(83)$ & $C(84)$ & $110(6)$ & $C(85)$ & $C(90)$ & $C(91)$ & $39(2)$ \\
\hline$C(76)$ & $C(84)$ & C(77) & $63(5)$ & C(89) & $C(90)$ & $C(91)$ & $103(3)$ \\
\hline$C(76)$ & $C(84)$ & $C(83)$ & $116(6)$ & $\mathrm{P}(43)$ & $C(91)$ & $C(85)$ & $120(5)$ \\
\hline$C(77)$ & $C(84)$ & $C(83)$ & $84(5)$ & $\mathrm{P}(43)$ & $C(91)$ & $C(86)$ & $138(3)$ \\
\hline $\mathrm{P}(32)$ & $C(85)$ & $C(86)$ & $123(3)$ & $\mathrm{P}(43)$ & $C(91)$ & $C(90)$ & $115(3)$ \\
\hline $\mathrm{P}(32)$ & $C(85)$ & $C(90)$ & $115(3)$ & $\mathrm{P}(43)$ & $C(91)$ & $C(92)$ & $124(3)$ \\
\hline $\mathrm{P}(32)$ & $C(85)$ & $C(91)$ & $104(5)$ & $C(85)$ & $C(91)$ & $C(86)$ & $53(4)$ \\
\hline $\mathrm{P}(32)$ & $C(85)$ & $C(92)$ & $123(2)$ & $C(85)$ & $C(91)$ & $C(90)$ & $67(3)$ \\
\hline$C(86)$ & $C(85)$ & $C(90)$ & $122(4)$ & $C(85)$ & $C(91)$ & $C(92)$ & $83(4)$ \\
\hline$C(86)$ & $C(85)$ & $C(91)$ & $90(4)$ & $C(86)$ & $C(91)$ & $C(90)$ & $100(3)$ \\
\hline$C(86)$ & $C(85)$ & C(92) & $31(3)$ & $C(86)$ & $C(91)$ & $C(92)$ & $31(2)$ \\
\hline$C(90)$ & $C(85)$ & $C(91)$ & $73(3)$ & $C(90)$ & $C(91)$ & $C(92)$ & $121(4)$ \\
\hline$C(90)$ & $C(85)$ & $C(92)$ & $111(3)$ & $C(85)$ & $C(92)$ & $C(86)$ & $53(4)$ \\
\hline$C(91)$ & $C(85)$ & $C(92)$ & $60(4)$ & $C(85)$ & $C(92)$ & $C(87)$ & 101(3) \\
\hline$C(85)$ & $C(86)$ & $C(87)$ & $121(3)$ & $C(85)$ & $C(92)$ & $C(91)$ & $37(3)$ \\
\hline$C(85)$ & $C(86)$ & $C(91)$ & $37(3)$ & $C(86)$ & $C(92)$ & $C(87)$ & $70(5)$ \\
\hline$C(85)$ & $C(86)$ & $C(92)$ & $96(5)$ & $C(86)$ & $C(92)$ & $C(91)$ & $90(5)$ \\
\hline$C(87)$ & $C(86)$ & $C(91)$ & $101(3)$ & C (87) & $C(92)$ & $C(91)$ & $115(4)$ \\
\hline$C(87)$ & $C(86)$ & $C(92)$ & $71(5)$ & $\mathrm{P}(12)$ & $C(93)$ & $\mathrm{P}(23)$ & $75(2)$ \\
\hline $\mathrm{P}(12)$ & $C(93)$ & $C(94)$ & $97(2)$ & $C(93)$ & $C(98)$ & $C(100)$ & $101(4)$ \\
\hline $\mathrm{P}(12)$ & $C(93)$ & C(98) & $104(3)$ & $C(97)$ & $C(98)$ & $C(99)$ & $89(2)$ \\
\hline $\mathrm{P}(12)$ & $C(93)$ & $C(99)$ & $56(3)$ & $\mathrm{C}(97)$ & $C(98)$ & $C(100)$ & $50(3)$ \\
\hline$P(23)$ & C(93) & $C(94)$ & $125(3)$ & C(99) & $C(98)$ & $C(100)$ & $64(3)$ \\
\hline$P(23)$ & $C(93)$ & C(98) & $114(3)$ & $\mathrm{P}(12)$ & $C(99)$ & $C(93)$ & $94(3)$ \\
\hline$P(23)$ & $C(93)$ & C(99) & $130(4)$ & $\mathrm{P}(12)$ & C(99) & $C(94)$ & $123(3)$ \\
\hline$C(94)$ & $C(93)$ & $C(98)$ & $121(4)$ & $\mathrm{P}(12)$ & $C(99)$ & $C(98)$ & $108(2)$ \\
\hline$C(94)$ & $C(93)$ & $C(99)$ & $61(2)$ & $\mathrm{P}(12)$ & $C(99)$ & $C(100)$ & $112(2)$ \\
\hline$C(98)$ & $C(93)$ & $C(99)$ & $87(4)$ & $C(93)$ & $C(99)$ & $C(94)$ & $73(3)$ \\
\hline C(93) & $C(94)$ & C(95) & $126(3)$ & C(93) & C(99) & C(98) & $55(3)$ \\
\hline
\end{tabular}




\begin{tabular}{|c|c|c|c|c|c|c|c|}
\hline$C(93)$ & $C(94)$ & $C(99)$ & $46(2)$ & $C(93)$ & C(99) & $C(100)$ & $94(3)$ \\
\hline$C(95)$ & $C(94)$ & C(99) & $115(3)$ & $C(94)$ & C(99) & $C(98)$ & $108(3)$ \\
\hline$C(94)$ & $C(95)$ & $C(96)$ & $109(2)$ & C(94) & C(99) & $C(100)$ & $124(3)$ \\
\hline C(94) & C(95) & $C(101)$ & $121(3)$ & $C(98)$ & C(99) & $C(100)$ & $40(2)$ \\
\hline$C(96)$ & C(95) & $C(101)$ & $27(2)$ & $\mathrm{C}(97)$ & $C(100)$ & $C(98)$ & $86(4)$ \\
\hline C(95) & $C(96)$ & $C(97)$ & $118(4)$ & $C(97)$ & $C(100)$ & $C(99)$ & $110(3)$ \\
\hline C(95) & C(96) & $C(101)$ & $61(4)$ & C(98) & $C(100)$ & C(99) & $77(3)$ \\
\hline$C(97)$ & $C(96)$ & $C(101)$ & $100(5)$ & $C(95)$ & $C(101)$ & $C(96)$ & $92(5)$ \\
\hline$C(96)$ & $C(97)$ & $C(98)$ & $124(4)$ & $C(95)$ & $C(101)$ & $C(97)$ & $111(4)$ \\
\hline$C(96)$ & C(97) & $C(100)$ & $108(4)$ & $C(96)$ & $C(101)$ & C(97) & $47(4)$ \\
\hline$C(96)$ & C(97) & $C(101)$ & $33(3)$ & $\mathrm{P}(31)$ & $C(102)$ & $C(103)$ & $118(2)$ \\
\hline$C(98)$ & $C(97)$ & $C(100)$ & $43(2)$ & $\mathrm{P}(31)$ & $C(102)$ & $C(107)$ & 119(3) \\
\hline C(98) & C(97) & $C(101)$ & $114(3)$ & $\mathrm{P}(31)$ & $C(102)$ & $C(108)$ & $113(4)$ \\
\hline$C(100)$ & C(97) & $C(101)$ & $126(3)$ & $\mathrm{P}(31)$ & $C(102)$ & $C(111)$ & $135(3)$ \\
\hline$C(93)$ & $C(98)$ & $C(97)$ & $111(3)$ & $C(103)$ & $C(102)$ & $C(107)$ & $122(3)$ \\
\hline$C(93)$ & $C(98)$ & C(99) & $38(2)$ & $C(103)$ & $C(102)$ & $C(108)$ & $57(4)$ \\
\hline$C(103)$ & $C(102)$ & $C(111)$ & $98(3)$ & $C(107)$ & $C(106)$ & $C(111)$ & $33(3)$ \\
\hline C(107) & $C(102)$ & $C(108)$ & $98(4)$ & $C(110)$ & $C(106)$ & $C(111)$ & $117(5)$ \\
\hline C(107) & $C(102)$ & $C(111)$ & $34(2)$ & $C(102)$ & $C(107)$ & $C(106)$ & $114(3)$ \\
\hline$C(108)$ & $C(102)$ & $C(111)$ & $65(3)$ & $C(102)$ & $C(107)$ & $C(108)$ & $34(2)$ \\
\hline$C(102)$ & $C(103)$ & $C(104)$ & $125(2)$ & $C(102)$ & $C(107)$ & $C(111)$ & $85(4)$ \\
\hline$C(102)$ & $C(103)$ & $C(108)$ & $44(3)$ & $C(106)$ & $C(107)$ & $C(108)$ & $91(3)$ \\
\hline$C(102)$ & $C(103)$ & $C(109)$ & $109(3)$ & $C(106)$ & $C(107)$ & $C(111)$ & $54(4)$ \\
\hline$C(104)$ & $\mathrm{C}(103)$ & $C(108)$ & $109(3)$ & $\mathrm{C}(108)$ & $C(107)$ & $\mathrm{C}(111)$ & $51(3)$ \\
\hline C(104) & $C(103)$ & $C(109)$ & $37(3)$ & $\mathrm{P}(42)$ & $C(108)$ & $C(102)$ & $113(4)$ \\
\hline$C(108)$ & $C(103)$ & $C(109)$ & $124(3)$ & $\mathrm{P}(42)$ & $C(108)$ & $C(103)$ & $124(3)$ \\
\hline$C(103)$ & $C(104)$ & $C(105)$ & $112(4)$ & $\mathrm{P}(42)$ & $C(108)$ & $C(107)$ & $122(3)$ \\
\hline$C(103)$ & $C(104)$ & $C(109)$ & $66(5)$ & $P(42)$ & $C(108)$ & $C(111)$ & $120(4)$ \\
\hline$C(103)$ & $C(104)$ & $C(110)$ & 99(4) & $C(102)$ & $C(108)$ & $C(103)$ & $80(4)$ \\
\hline$C(105)$ & $C(104)$ & $C(109)$ & $97(4)$ & $C(102)$ & $C(108)$ & $C(107)$ & $48(3)$ \\
\hline$C(105)$ & $C(104)$ & $C(110)$ & $32(3)$ & $C(102)$ & $C(108)$ & $C(111)$ & $76(3)$ \\
\hline C(109) & $C(104)$ & $C(110)$ & $65(4)$ & $C(103)$ & $C(108)$ & $C(107)$ & $107(3)$ \\
\hline$C(104)$ & $C(105)$ & $C(106)$ & $125(4)$ & $C(103)$ & $C(108)$ & $C(111)$ & $116(4)$ \\
\hline$C(104)$ & $C(105)$ & $C(109)$ & $28(2)$ & $C(107)$ & $C(108)$ & $C(111)$ & $28(2)$ \\
\hline$C(104)$ & $C(105)$ & $C(110)$ & $96(5)$ & $C(103)$ & $C(109)$ & $C(104)$ & $77(5)$ \\
\hline$C(106)$ & $C(105)$ & $C(109)$ & $105(3)$ & $C(103)$ & $C(109)$ & $C(105)$ & $99(3)$ \\
\hline$C(106)$ & $C(105)$ & $C(110)$ & $59(6)$ & $C(103)$ & $C(109)$ & $C(110)$ & $113(5)$ \\
\hline C(109) & $C(105)$ & $C(110)$ & $68(5)$ & $C(104)$ & $C(109)$ & $C(105)$ & $55(4)$ \\
\hline$C(105)$ & $C(106)$ & $C(107)$ & $121(3)$ & $C(104)$ & $C(109)$ & $C(110)$ & $88(5)$ \\
\hline$C(105)$ & $C(106)$ & $C(110)$ & 49(5) & $C(105)$ & $C(109)$ & $C(110)$ & $33(3)$ \\
\hline$C(105)$ & $C(106)$ & $C(111)$ & 102(3) & $C(104)$ & $C(110)$ & $C(105)$ & $51(4)$ \\
\hline$C(107)$ & $C(106)$ & $C(110)$ & $106(5)$ & $C(104)$ & $C(110)$ & $C(106)$ & $105(6)$ \\
\hline$C(104)$ & $C(110)$ & $C(109)$ & $27(2)$ & $\mathrm{P}(31)$ & $C(33)$ & $C(38)$ & $97(2)$ \\
\hline$C(105)$ & $C(110)$ & $C(106)$ & 72(7) & $\mathrm{P}(41)$ & $C(33)$ & $C(34)$ & $103(2)$ \\
\hline$C(105)$ & $C(110)$ & $C(109)$ & 78(5) & $\mathrm{P}(41)$ & $C(33)$ & $C(38)$ & $134(2)$ \\
\hline$C(106)$ & $C(110)$ & $C(109)$ & $118(7)$ & $C(34)$ & $C(33)$ & $C(38)$ & $120(2)$ \\
\hline$C(102)$ & $C(111)$ & $C(106)$ & $122(4)$ & C(33) & $C(34)$ & $C(35)$ & $120(3)$ \\
\hline$C(102)$ & $C(111)$ & $C(107)$ & 62(4) & $C(34)$ & $C(35)$ & $C(36)$ & $120(3)$ \\
\hline$C(102)$ & $C(111)$ & $C(108)$ & $39(2)$ & C(35) & $C(36)$ & C(37) & $120(2)$ \\
\hline$C(106)$ & $C(111)$ & $C(107)$ & $94(5)$ & $C(36)$ & $C(37)$ & C(38) & $120(3)$ \\
\hline$C(106)$ & $C(111)$ & $C(108)$ & $127(5)$ & $C(33)$ & $C(38)$ & $C(37)$ & $120(3)$ \\
\hline$C(107)$ & $C(111)$ & $C(108)$ & $101(5)$ & $\mathrm{P}(21)$ & $C(27)$ & $C(32)$ & $115(1)$ \\
\hline $\mathrm{Cl}(3)$ & $C(201)$ & $\mathrm{Cl}(4)$ & $118(3)$ & $\mathrm{P}(21)$ & $C(27)$ & $C(28)$ & $125(2)$ \\
\hline $\mathrm{Cl}(1)$ & $C(202)$ & $\mathrm{Cl}(2)$ & $110(2)$ & $C(32)$ & $C(27)$ & $C(28)$ & $120(2)$ \\
\hline $\mathrm{Cl}(5)$ & $C(203)$ & $\mathrm{Cl}(6)$ & $118(4)$ & $C(27)$ & $C(32)$ & $C(31)$ & $120(2)$ \\
\hline $\mathrm{P}(11)$ & $C(21)$ & $\mathrm{P}(21)$ & $39.7(6)$ & $C(32)$ & $C(31)$ & C(30) & $120(2)$ \\
\hline
\end{tabular}




\begin{tabular}{|c|c|c|c|c|c|c|c|}
\hline$P(11)$ & $C(21)$ & $C(22)$ & $114(1)$ & $C(31)$ & $C(30)$ & $C(29)$ & $120(2)$ \\
\hline $\mathrm{P}(11)$ & $C(21)$ & $C(26)$ & $123(1)$ & $C(30)$ & $C(29)$ & $C(28)$ & $120(2)$ \\
\hline $\mathrm{P}(21)$ & $C(21)$ & $C(22)$ & $154(1)$ & $C(27)$ & C(28) & $C(29)$ & $120(2)$ \\
\hline $\mathrm{P}(21)$ & $C(21)$ & $C(26)$ & $85(1)$ & $\mathrm{P}(41)$ & $C(39)$ & $C(44)$ & $119(2)$ \\
\hline$C(22)$ & $C(21)$ & $C(26)$ & $120(1)$ & $\mathrm{P}(41)$ & $C(39)$ & $C(40)$ & $121(2)$ \\
\hline$C(21)$ & $C(22)$ & $C(23)$ & $120(2)$ & $C(44)$ & C(39) & $C(40)$ & $120(3)$ \\
\hline$C(22)$ & $C(23)$ & $C(24)$ & $120(2)$ & $C(39)$ & $C(44)$ & $C(43)$ & $120(3)$ \\
\hline$C(23)$ & $C(24)$ & $C(25)$ & $120(2)$ & $C(44)$ & $C(43)$ & $C(42)$ & $120(2)$ \\
\hline$C(24)$ & $C(25)$ & $C(26)$ & $120(2)$ & $C(43)$ & $C(42)$ & $C(41)$ & $120(3)$ \\
\hline$C(21)$ & $C(26)$ & $C(25)$ & $120(2)$ & $C(42)$ & $C(41)$ & $C(40)$ & $120(3)$ \\
\hline $\mathrm{P}(31)$ & $C(33)$ & $\mathrm{P}(41)$ & $39.3(6)$ & $C(39)$ & $C(40)$ & $C(41)$ & $120(2)$ \\
\hline $\mathrm{P}(31)$ & $C(33)$ & $C(34)$ & $142(2)$ & $\mathrm{P}(33)$ & $C(45)$ & $C(46)$ & 121(1) \\
\hline $\mathrm{P}(33)$ & $C(45)$ & $C(50)$ & $119(2)$ & $\mathrm{C}(74)$ & $C(69)$ & $C(68)$ & $27(2)$ \\
\hline$C(46)$ & $C(45)$ & $C(50)$ & $120(2)$ & $C(63)$ & $C(69)$ & $C(70)$ & $45(2)$ \\
\hline$C(45)$ & $C(46)$ & $C(47)$ & $120(2)$ & $C(63)$ & $C(69)$ & $C(68)$ & $164(4)$ \\
\hline$C(46)$ & $C(47)$ & $C(48)$ & $120(2)$ & $C(70)$ & $C(69)$ & $C(68)$ & $138(3)$ \\
\hline$C(47)$ & $C(48)$ & $C(49)$ & $120(2)$ & $C(69)$ & $C(64)$ & $\mathrm{C}(71)$ & $120(2)$ \\
\hline$C(48)$ & $C(49)$ & $C(50)$ & $120(2)$ & $C(69)$ & $C(64)$ & $C(63)$ & $24(2)$ \\
\hline$C(45)$ & $C(50)$ & $C(49)$ & $120(2)$ & $C(69)$ & $C(64)$ & $C(70)$ & $117(3)$ \\
\hline $\mathrm{P}(13)$ & $C(51)$ & $C(52)$ & $127(1)$ & $C(69)$ & $C(64)$ & $C(65)$ & $109(2)$ \\
\hline $\mathrm{P}(13)$ & $C(51)$ & $C(56)$ & $113(1)$ & $\mathrm{C}(71)$ & $C(64)$ & $C(63)$ & $144(3)$ \\
\hline$C(52)$ & $C(51)$ & $C(56)$ & $120(2)$ & $C(71)$ & $C(64)$ & $C(70)$ & $75(3)$ \\
\hline$C(51)$ & $C(52)$ & $C(53)$ & $120(1)$ & $\mathrm{C}(71)$ & $C(64)$ & $C(65)$ & $27(1)$ \\
\hline$C(52)$ & $C(53)$ & $C(54)$ & $120(2)$ & $C(63)$ & $C(64)$ & $C(70)$ & $112(3)$ \\
\hline$C(53)$ & $C(54)$ & $C(55)$ & $120(2)$ & $C(63)$ & $C(64)$ & $C(65)$ & $127(2)$ \\
\hline$C(54)$ & $C(55)$ & $C(56)$ & $120(1)$ & $\mathrm{C}(70)$ & $C(64)$ & $C(65)$ & $56(3)$ \\
\hline$C(51)$ & $C(56)$ & $C(55)$ & $120(2)$ & $C(64)$ & $C(71)$ & $\mathrm{C}(72)$ & $120(2)$ \\
\hline $\mathrm{P}(23)$ & $C(69)$ & C(64) & $125(2)$ & $C(64)$ & $C(71)$ & $C(70)$ & $26(2)$ \\
\hline $\mathrm{P}(23)$ & $C(69)$ & $C(74)$ & $115(2)$ & $C(64)$ & $C(71)$ & $C(65)$ & $95(3)$ \\
\hline $\mathrm{P}(23)$ & $C(69)$ & $C(63)$ & $82(3)$ & $C(64)$ & $C(71)$ & $C(66)$ & $103(2)$ \\
\hline $\mathrm{P}(23)$ & $C(69)$ & $C(70)$ & $124(2)$ & $C(72)$ & $C(71)$ & $C(70)$ & $126(2)$ \\
\hline $\mathrm{P}(23)$ & $C(69)$ & $C(68)$ & $98(2)$ & $C(72)$ & $C(71)$ & $C(65)$ & $71(3)$ \\
\hline$C(64)$ & $C(69)$ & $C(74)$ & $120(2)$ & $C(72)$ & $C(71)$ & $C(66)$ & 20(1) \\
\hline$C(64)$ & $C(69)$ & $C(63)$ & $43(2)$ & $\mathrm{C}(70)$ & $C(71)$ & $C(65)$ & $76(3)$ \\
\hline$C(64)$ & $C(69)$ & $C(70)$ & $18(1)$ & $C(70)$ & $C(71)$ & $C(66)$ & $106(2)$ \\
\hline$C(64)$ & $C(69)$ & $C(68)$ & $132(3)$ & $C(65)$ & $C(71)$ & $C(66)$ & $58(3)$ \\
\hline$C(74)$ & $C(69)$ & $C(63)$ & $162(3)$ & $\mathrm{C}(71)$ & $\mathrm{C}(72)$ & $C(73)$ & $120(2)$ \\
\hline$C(74)$ & $C(69)$ & $C(70)$ & $118(2)$ & $\mathrm{C}(71)$ & $C(72)$ & $C(65)$ & $32(2)$ \\
\hline $\mathrm{C}(71)$ & $\mathrm{C}(72)$ & $C(66)$ & 104(5) & $\mathrm{P}(23)$ & $C(63)$ & $C(64)$ & $167(2)$ \\
\hline $\mathrm{C}(71)$ & $C(72)$ & $C(67)$ & 102(2) & $\mathrm{P}(23)$ & $C(63)$ & $C(70)$ & $155(2)$ \\
\hline$C(73)$ & $C(72)$ & $C(65)$ & $113(2)$ & $\mathrm{P}(23)$ & $C(63)$ & $C(68)$ & $79(1)$ \\
\hline$C(73)$ & $C(72)$ & $C(66)$ & $39(4)$ & $C(69)$ & $C(63)$ & $C(64)$ & 113(3) \\
\hline$C(73)$ & $C(72)$ & $C(67)$ & $18(1)$ & $C(69)$ & $C(63)$ & $C(70)$ & $118(3)$ \\
\hline$C(65)$ & $C(72)$ & $C(66)$ & $82(5)$ & $C(69)$ & $C(63)$ & $C(68)$ & $9(2)$ \\
\hline$C(65)$ & $C(72)$ & $C(67)$ & $100(2)$ & $C(64)$ & $C(63)$ & $C(70)$ & $24(2)$ \\
\hline$C(66)$ & $C(72)$ & $C(67)$ & $42(3)$ & $C(64)$ & $C(63)$ & $C(68)$ & 111(2) \\
\hline$C(72)$ & $C(73)$ & $C(74)$ & $120(3)$ & $\mathrm{C}(70)$ & $C(63)$ & $C(68)$ & $120(2)$ \\
\hline $\mathrm{C}(72)$ & $C(73)$ & $C(66)$ & $22(2)$ & $C(69)$ & $C(70)$ & $C(64)$ & $45(2)$ \\
\hline$C(72)$ & $C(73)$ & $C(67)$ & $117(4)$ & $C(69)$ & $C(70)$ & $C(71)$ & $100(2)$ \\
\hline$C(74)$ & $C(73)$ & $C(66)$ & $112(3)$ & $C(69)$ & $C(70)$ & $C(63)$ & $18(1)$ \\
\hline$C(74)$ & $C(73)$ & $C(67)$ & 13(3) & $C(69)$ & $C(70)$ & $C(65)$ & $102(2)$ \\
\hline$C(66)$ & $C(73)$ & $\mathrm{C}(67)$ & $115(5)$ & $C(64)$ & $C(70)$ & $\mathrm{C}(71)$ & $79(3)$ \\
\hline C(69) & $C(74)$ & $C(73)$ & $120(2)$ & $C(64)$ & $C(70)$ & $C(63)$ & $43(2)$ \\
\hline$C(69)$ & $C(74)$ & $C(67)$ & $118(3)$ & $C(64)$ & $C(70)$ & $C(65)$ & $102(4)$ \\
\hline$C(69)$ & $C(74)$ & $C(68)$ & $28(2)$ & $\mathrm{C}(71)$ & $C(70)$ & $C(63)$ & $113(2)$ \\
\hline$C(73)$ & $C(74)$ & $C(67)$ & $10(2)$ & $\mathrm{C}(71)$ & $C(70)$ & $C(65)$ & $31(2)$ \\
\hline
\end{tabular}




\begin{tabular}{|c|c|c|c|c|c|c|c|}
\hline $\mathrm{C}(73)$ & $\mathrm{C}(74)$ & $C(68)$ & $132(3)$ & $C(63)$ & $C(70)$ & $C(65)$ & $120(2)$ \\
\hline$C(67)$ & $C(74)$ & C(68) & $125(3)$ & $C(64)$ & $C(65)$ & $\mathrm{C}(71)$ & $58(2)$ \\
\hline $\mathrm{P}(13)$ & $C(63)$ & $P(23)$ & 41.1(7) & $C(64)$ & $C(65)$ & $C(72)$ & $107(2)$ \\
\hline $\mathrm{P}(13)$ & $C(63)$ & C(69) & $119(3)$ & $C(64)$ & $C(65)$ & $\mathrm{C}(70)$ & $22(1)$ \\
\hline $\mathrm{P}(13)$ & $C(63)$ & $C(64)$ & $128(2)$ & $C(64)$ & $C(65)$ & $C(66)$ & $103(2)$ \\
\hline $\mathrm{P}(13)$ & $C(63)$ & $\mathrm{C}(70)$ & $117(2)$ & $\mathrm{C}(71)$ & $C(65)$ & $\mathrm{C}(72)$ & 77(4) \\
\hline $\mathrm{P}(13)$ & $C(63)$ & $C(68)$ & $120(1)$ & $\mathrm{C}(71)$ & $C(65)$ & $\mathrm{C}(70)$ & 73(3) \\
\hline $\mathrm{P}(23)$ & $C(63)$ & $C(69)$ & $79(2)$ & $\mathrm{C}(71)$ & $C(65)$ & $C(66)$ & 95(4) \\
\hline$C(72)$ & $C(65)$ & $\mathrm{C}(70)$ & $128(2)$ & $P(23)$ & $C(68)$ & $C(67)$ & $165(2)$ \\
\hline $\mathrm{C}(72)$ & $C(65)$ & $C(66)$ & $25(1)$ & $C(69)$ & $C(68)$ & $C(74)$ & $126(4)$ \\
\hline$C(70)$ & $C(65)$ & $C(66)$ & $120(2)$ & $C(69)$ & $C(68)$ & $C(63)$ & $7(2)$ \\
\hline C(71) & $C(66)$ & $C(72)$ & $56(4)$ & $C(69)$ & $C(68)$ & $C(67)$ & $118(3)$ \\
\hline$C(71)$ & $C(66)$ & $C(73)$ & $130(2)$ & $C(74)$ & $C(68)$ & $C(63)$ & 131(3) \\
\hline$C(71)$ & $C(66)$ & $C(65)$ & $27(1)$ & $C(74)$ & $C(68)$ & $C(67)$ & $28(2)$ \\
\hline $\mathrm{C}(71)$ & $C(66)$ & $C(67)$ & $109(2)$ & $C(63)$ & $C(68)$ & $C(67)$ & $120(2)$ \\
\hline $\mathrm{C}(72)$ & $C(66)$ & $\mathrm{C}(73)$ & $120(5)$ & $\mathrm{P}(33)$ & $C(57)$ & $\mathrm{P}(43)$ & $41.8(7)$ \\
\hline$C(72)$ & $C(66)$ & $C(65)$ & 74(5) & $\mathrm{P}(33)$ & $C(57)$ & $C(62)$ & $119(2)$ \\
\hline $\mathrm{C}(72)$ & $C(66)$ & $C(67)$ & $121(4)$ & $\mathrm{P}(33)$ & $C(57)$ & $C(58)$ & $119(2)$ \\
\hline C(73) & $C(66)$ & $C(65)$ & $144(3)$ & $\mathrm{P}(33)$ & $C(57)$ & $C(112)$ & $117(10)$ \\
\hline$C(73)$ & $C(66)$ & $C(67)$ & $24(2)$ & $\mathrm{P}(33)$ & $C(57)$ & $C(114)$ & $120(5)$ \\
\hline$C(65)$ & $C(66)$ & $C(67)$ & $120(2)$ & $\mathrm{P}(33)$ & $C(57)$ & $C(113)$ & $128(3)$ \\
\hline $\mathrm{C}(72)$ & $C(67)$ & $\mathrm{C}(73)$ & $44(3)$ & $\mathrm{P}(43)$ & $C(57)$ & $C(62)$ & $159(2)$ \\
\hline$C(72)$ & $C(67)$ & $C(74)$ & $137(3)$ & $\mathrm{P}(43)$ & $C(57)$ & $C(58)$ & $77(2)$ \\
\hline$C(72)$ & $C(67)$ & $C(66)$ & $16(1)$ & $\mathrm{P}(43)$ & $C(57)$ & $C(112)$ & $78(11)$ \\
\hline $\mathrm{C}(72)$ & $C(67)$ & $C(68)$ & $118(2)$ & $\mathrm{P}(43)$ & $C(57)$ & $C(114)$ & $142(4)$ \\
\hline$C(73)$ & $C(67)$ & $C(74)$ & $156(4)$ & $\mathrm{P}(43)$ & $C(57)$ & $C(113)$ & $89(3)$ \\
\hline C(73) & $C(67)$ & $C(66)$ & $41(3)$ & $C(62)$ & $C(57)$ & $C(58)$ & $120(2)$ \\
\hline$C(73)$ & $C(67)$ & $C(68)$ & $161(4)$ & $C(62)$ & $C(57)$ & $C(112)$ & $115(11)$ \\
\hline $\mathrm{C}(74)$ & $C(67)$ & $C(66)$ & 131(4) & $C(62)$ & $C(57)$ & $C(114)$ & $47(4)$ \\
\hline $\mathrm{C}(74)$ & $C(67)$ & $C(68)$ & $27(2)$ & $C(62)$ & $C(57)$ & $C(113)$ & $105(3)$ \\
\hline$C(66)$ & $C(67)$ & $C(68)$ & $120(2)$ & $C(58)$ & $C(57)$ & $C(112)$ & $16(11)$ \\
\hline $\mathrm{P}(23)$ & $C(68)$ & $C(69)$ & $60(2)$ & $C(58)$ & $C(57)$ & $C(114)$ & $112(5)$ \\
\hline $\mathrm{P}(23)$ & $C(68)$ & $\mathrm{C}(74)$ & $140(2)$ & C(58) & C(57) & $C(113)$ & $18(3)$ \\
\hline $\mathrm{P}(23)$ & $C(68)$ & $C(63)$ & 60(1) & $C(112)$ & $C(57)$ & $C(114)$ & $120(11)$ \\
\hline$C(112)$ & $C(57)$ & $C(113)$ & 11(10) & $C(117)$ & $C(59)$ & $C(113)$ & $146(13)$ \\
\hline$C(114)$ & C(57) & $C(113)$ & 110(5) & $\mathrm{P}(43)$ & $C(58)$ & C(57) & 63(1) \\
\hline C(57) & $C(62)$ & $C(61)$ & $120(3)$ & $\mathrm{P}(43)$ & $C(58)$ & $C(59)$ & $169(2)$ \\
\hline$C(57)$ & $C(62)$ & $C(112)$ & 11(3) & $\mathrm{P}(43)$ & $C(58)$ & $C(112)$ & $64(3)$ \\
\hline C(57) & $C(62)$ & $C(114)$ & $56(4)$ & $\mathrm{P}(43)$ & $C(58)$ & $C(117)$ & $178(4)$ \\
\hline$C(61)$ & $C(62)$ & $C(112)$ & $109(4)$ & $\mathrm{P}(43)$ & $C(58)$ & $C(113)$ & $135(8)$ \\
\hline$C(61)$ & $C(62)$ & $C(114)$ & $108(5)$ & $C(57)$ & $C(58)$ & C(59) & $120(2)$ \\
\hline$C(112)$ & $C(62)$ & $C(114)$ & $61(5)$ & $C(57)$ & $C(58)$ & $C(112)$ & $5(3)$ \\
\hline$C(62)$ & $C(61)$ & $C(60)$ & $120(2)$ & $C(57)$ & $C(58)$ & $C(117)$ & $118(4)$ \\
\hline$C(62)$ & $C(61)$ & $C(115)$ & $73(3)$ & $C(57)$ & $C(58)$ & $C(113)$ & $115(10)$ \\
\hline$C(60)$ & $C(61)$ & $C(115)$ & $86(4)$ & C(59) & $C(58)$ & $C(112)$ & $119(4)$ \\
\hline$C(61)$ & $C(60)$ & $C(59)$ & $120(3)$ & $C(59)$ & $C(58)$ & $C(117)$ & $13(3)$ \\
\hline$C(61)$ & $C(60)$ & $C(116)$ & $92(7)$ & $C(59)$ & $C(58)$ & $C(113)$ & $34(8)$ \\
\hline$C(61)$ & $C(60)$ & $C(117)$ & $128(5)$ & $C(112)$ & $C(58)$ & $C(117)$ & $117(5)$ \\
\hline C(59) & $C(60)$ & $C(116)$ & 79(5) & $C(112)$ & $C(58)$ & $C(113)$ & $111(11)$ \\
\hline C(59) & $C(60)$ & $C(117)$ & $20(4)$ & $\mathrm{C}(117)$ & $C(58)$ & $C(113)$ & $47(9)$ \\
\hline$C(116)$ & $C(60)$ & $C(117)$ & $63(6)$ & $\mathrm{P}(33)$ & $C(112)$ & $\mathrm{P}(43)$ & $40(1)$ \\
\hline$C(60)$ & $C(59)$ & $C(58)$ & $120(3)$ & $\mathrm{P}(33)$ & $C(112)$ & C(57) & $55(10)$ \\
\hline$C(60)$ & $C(59)$ & $C(116)$ & $50(3)$ & $\mathrm{P}(33)$ & $C(112)$ & $C(62)$ & 102(5) \\
\hline$C(60)$ & $C(59)$ & $C(117)$ & 68(13) & $\mathrm{P}(33)$ & $C(112)$ & $C(58)$ & $126(5)$ \\
\hline$C(60)$ & $C(59)$ & $C(113)$ & $118(6)$ & $\mathrm{P}(33)$ & $C(112)$ & $C(114)$ & $100(6)$ \\
\hline$C(58)$ & C(59) & $C(116)$ & $106(4)$ & $\mathrm{P}(33)$ & $C(112)$ & $C(113)$ & $139(6)$ \\
\hline
\end{tabular}




\begin{tabular}{lllrllll}
$\mathrm{C}(58)$ & $\mathrm{C}(59)$ & $\mathrm{C}(117)$ & $126(11)$ & $\mathrm{P}(43)$ & $\mathrm{C}(112)$ & $\mathrm{C}(57)$ & $92(11)$ \\
$\mathrm{C}(58)$ & $\mathrm{C}(59)$ & $\mathrm{C}(113)$ & $20(5)$ & $\mathrm{P}(43)$ & $\mathrm{C}(112)$ & $\mathrm{C}(62)$ & $142(6)$ \\
$\mathrm{C}(116)$ & $\mathrm{C}(59)$ & $\mathrm{C}(117)$ & $34(15)$ & $\mathrm{P}(43)$ & $\mathrm{C}(112)$ & $\mathrm{C}(58)$ & $86(5)$ \\
$\mathrm{C}(116)$ & $\mathrm{C}(59)$ & $\mathrm{C}(113)$ & $121(6)$ & $\mathrm{P}(43)$ & $\mathrm{C}(112)$ & $\mathrm{C}(114)$ & $128(6)$ \\
& & & & & & & \\
$\mathrm{P}(43)$ & $\mathrm{C}(112)$ & $\mathrm{C}(113)$ & $101(6)$ & $\mathrm{C}(60)$ & $\mathrm{C}(117)$ & $\mathrm{C}(59)$ & $92(15)$ \\
$\mathrm{C}(57)$ & $\mathrm{C}(112)$ & $\mathrm{C}(62)$ & $54(11)$ & $\mathrm{C}(60)$ & $\mathrm{C}(117)$ & $\mathrm{C}(58)$ & $106(8)$ \\
$\mathrm{C}(57)$ & $\mathrm{C}(112)$ & $\mathrm{C}(58)$ & $159(13)$ & $\mathrm{C}(60)$ & $\mathrm{C}(117)$ & $\mathrm{C}(116)$ & $61(6)$ \\
$\mathrm{C}(57)$ & $\mathrm{C}(112)$ & $\mathrm{C}(114)$ & $47(10)$ & $\mathrm{C}(60)$ & $\mathrm{C}(117)$ & $\mathrm{C}(113)$ & $99(9)$ \\
$\mathrm{C}(57)$ & $\mathrm{C}(112)$ & $\mathrm{C}(113)$ & $167(13)$ & $\mathrm{C}(59)$ & $\mathrm{C}(117)$ & $\mathrm{C}(58)$ & $41(9)$ \\
$\mathrm{C}(62)$ & $\mathrm{C}(112)$ & $\mathrm{C}(58)$ & $132(5)$ & $\mathrm{C}(59)$ & $\mathrm{C}(117)$ & $\mathrm{C}(116)$ & $134(20)$ \\
$\mathrm{C}(62)$ & $\mathrm{C}(112)$ & $\mathrm{C}(114)$ & $41(4)$ & $\mathrm{C}(59)$ & $\mathrm{C}(117)$ & $\mathrm{C}(113)$ & $23(9)$ \\
$\mathrm{C}(62)$ & $\mathrm{C}(112)$ & $\mathrm{C}(113)$ & $114(5)$ & $\mathrm{C}(58)$ & $\mathrm{C}(117)$ & $\mathrm{C}(116)$ & $108(8)$ \\
$\mathrm{C}(58)$ & $\mathrm{C}(112)$ & $\mathrm{C}(114)$ & $120(7)$ & $\mathrm{C}(58)$ & $\mathrm{C}(117)$ & $\mathrm{C}(113)$ & $18(3)$ \\
$\mathrm{C}(58)$ & $\mathrm{C}(112)$ & $\mathrm{C}(113)$ & $23(4)$ & $\mathrm{C}(116)$ & $\mathrm{C}(117)$ & $\mathrm{C}(113)$ & $120(10)$ \\
$\mathrm{C}(114)$ & $\mathrm{C}(112)$ & $\mathrm{C}(113)$ & $120(7)$ & $\mathrm{C}(57)$ & $\mathrm{C}(113)$ & $\mathrm{C}(59)$ & $125(6)$ \\
$\mathrm{C}(57)$ & $\mathrm{C}(114)$ & $\mathrm{C}(62)$ & $77(4)$ & $\mathrm{C}(57)$ & $\mathrm{C}(113)$ & $\mathrm{C}(58)$ & $47(8)$ \\
$\mathrm{C}(57)$ & $\mathrm{C}(114)$ & $\mathrm{C}(112)$ & $12(4)$ & $\mathrm{C}(57)$ & $\mathrm{C}(113)$ & $\mathrm{C}(112)$ & $3(3)$ \\
$\mathrm{C}(57)$ & $\mathrm{C}(114)$ & $\mathrm{C}(115)$ & $132(9)$ & $\mathrm{C}(57)$ & $\mathrm{C}(113)$ & $\mathrm{C}(117)$ & $118(7)$ \\
$\mathrm{C}(62)$ & $\mathrm{C}(114)$ & $\mathrm{C}(112)$ & $79(5)$ & $\mathrm{C}(59)$ & $\mathrm{C}(113)$ & $\mathrm{C}(58)$ & $126(11)$ \\
$\mathrm{C}(62)$ & $\mathrm{C}(114)$ & $\mathrm{C}(115)$ & $99(7)$ & $\mathrm{C}(59)$ & $\mathrm{C}(113)$ & $\mathrm{C}(112)$ & $128(7)$ \\
$\mathrm{C}(112)$ & $\mathrm{C}(114)$ & $\mathrm{C}(115)$ & $120(9)$ & $\mathrm{C}(59)$ & $\mathrm{C}(113)$ & $\mathrm{C}(117)$ & $11(4)$ \\
$\mathrm{C}(61)$ & $\mathrm{C}(115)$ & $\mathrm{C}(114)$ & $78(5)$ & $\mathrm{C}(58)$ & $\mathrm{C}(113)$ & $\mathrm{C}(112)$ & $46(8)$ \\
$\mathrm{C}(61)$ & $\mathrm{C}(115)$ & $\mathrm{C}(116)$ & $78(5)$ & $\mathrm{C}(58)$ & $\mathrm{C}(113)$ & $\mathrm{C}(117)$ & $115(10)$ \\
$\mathrm{C}(114)$ & $\mathrm{C}(115)$ & $\mathrm{C}(116)$ & $120(8)$ & $\mathrm{C}(112)$ & $\mathrm{C}(113)$ & $\mathrm{C}(117)$ & $120(7)$ \\
$\mathrm{C}(60)$ & $\mathrm{C}(116)$ & $\mathrm{C}(59)$ & $51(3)$ & & & & \\
$\mathrm{C}(60)$ & $\mathrm{C}(116)$ & $\mathrm{C}(115)$ & $101(8)$ & & & & \\
$\mathrm{C}(60)$ & $\mathrm{C}(116)$ & $\mathrm{C}(117)$ & $56(5)$ & & & & \\
$\mathrm{C}(59)$ & $\mathrm{C}(116)$ & $\mathrm{C}(115)$ & $110(6)$ & & & & \\
$\mathrm{C}(59)$ & $\mathrm{C}(116)$ & $\mathrm{C}(117)$ & $11(5)$ & & & & \\
$\mathrm{C}(115)$ & $\mathrm{C}(116)$ & $\mathrm{C}(117)$ & $120(8)$ & & & & \\
\hline & & & &
\end{tabular}

a Estimated deviations are given in parentheses. 
Table S7-1. Crystallographic and Experimental Data for $9 \mathbf{b} \cdot 5 \mathrm{CH}_{2} \mathrm{Cl}_{2}$

\begin{tabular}{|c|c|}
\hline formula & $\mathrm{C}_{87} \mathrm{H}_{87} \mathrm{Cl}_{10} \mathrm{~F}_{18} \mathrm{~N}_{2} \mathrm{P}_{9} \mathrm{Pt}_{3}$ \\
\hline formula weight & 2721.19 \\
\hline color & dark blue \\
\hline size, $\mathrm{mm}$ & $0.35 \times 0.18 \times 0.10$ \\
\hline cryst. system & triclinic \\
\hline space group & P1- (No. 2) \\
\hline no. refs used for cell determination & $13372\left(6<2 \theta<55^{\circ}\right)$ \\
\hline \multirow[t]{7}{*}{ lattice const. } & $a=14.472(2) \AA$ \\
\hline & $b=19.286(2) \AA$ \\
\hline & $c=19.696(2) \AA$ \\
\hline & $\alpha=95.347(3)^{\circ}$ \\
\hline & $\beta=95.716(4)^{\circ}$ \\
\hline & $\gamma=100.407(6)^{\circ}$ \\
\hline & $V=5345.1(9) \AA^{3}$ \\
\hline Z & 2 \\
\hline$D_{\text {calc }}, \mathrm{g} \mathrm{cm}^{-3}$ & 1.691 \\
\hline$F(000)$ & 2648 \\
\hline$\mu(\operatorname{Mo} K \alpha), \mathrm{cm}^{-1}$ & 43.58 \\
\hline \multirow[b]{2}{*}{ diffractometer } & rrection was applied (multi-scan spherical method). \\
\hline & $\begin{array}{l}\text { Rigaku/MSC Mercury CCD } \\
(50 \mathrm{kV} 190 \mathrm{~mA})\end{array}$ \\
\hline radiation & $\operatorname{Mo} K \alpha(\lambda=0.71069 \AA)$ \\
\hline monochromator & graphite \\
\hline temp, ${ }^{\circ} \mathrm{C}$ & -120 \\
\hline $2 \theta$ range $^{\circ}$ & $6<2 \theta<55$ \\
\hline detector aperture, $\mathrm{mm}$ & $70 \times 70$ \\
\hline data images & 2160 \\
\hline$\omega$ oscillation range $(\chi=45, \phi=90), \circ$ & $-62-118$ \\
\hline exposure rate, ${ }^{\circ} \mathrm{sec}^{-1}$ & 120 \\
\hline detector swing angle, ${ }^{\circ}$ & 28 \\
\hline detector position, $\mathrm{mm}$ & 60 \\
\hline solution & Direct Methods (dirdif94 patty) \\
\hline refinement & full-matrix least-squares (teXsan) \\
\hline & $\begin{array}{l}\text { The } \mathrm{Pt}, \mathrm{Cl}, \mathrm{P} \text {, and } \mathrm{F} \text { atoms were refined with } \\
\text { anisotropic temperature factors, and other non- } \mathrm{H} \\
\text { atoms were refined isotropically. }\end{array}$ \\
\hline hydrogen atoms & $\begin{array}{l}\text { The H83 was determined by difference Fourier } \\
\text { synthesis and other } \mathrm{H} \text { atoms except for solvent } \\
\text { molecules were calcd with } \mathrm{C}-\mathrm{H}=0.95 \AA \text { A. All H } \\
\text { atoms were not refined. }\end{array}$ \\
\hline function minimized & $\Sigma w\left(\left|F_{o}\right|-\left|F_{c}\right|\right)^{2}$ \\
\hline weighting scheme & $1 / \sigma^{2}(F o)$ \\
\hline anomalous dispersion & all non-hydrogen atoms \\
\hline no. unique reflections & 23648 \\
\hline$R_{\text {int }}$ & 0.052 \\
\hline no. obsd. unique reflections & $16146(I>3 \sigma(I))$ \\
\hline$p$-factor & 0.03 \\
\hline no. variables & 718 \\
\hline data / param ratio & 22.49 \\
\hline$R^{\mathrm{a}}$ & 0.074 \\
\hline$R_{\mathrm{W}} \mathrm{b}$ & 0.090 \\
\hline GOF $\mathrm{C}$ & 2.43 \\
\hline$\rho_{\max }, \mathrm{e}^{-3}$ & $2.61,-2.15$ \\
\hline
\end{tabular}


${ }^{\mathrm{c}} \mathrm{GOF}=\left[\Sigma w\left(\left|F_{\mathrm{O}}\right|-\left|F_{\mathrm{C}}\right|\right)^{2} /\left(N_{\mathrm{O}}-N_{\mathrm{p}}\right]^{1 / 2}\left(N_{\mathrm{O}}=\right.\right.$ no. data, $N_{\mathrm{p}}=$ no. variables $)$.

Table S7-2. Final Positional Parameters and $B_{\text {eq }}$ for $9 \mathbf{b} \cdot 5 \mathrm{CH}_{2} \mathrm{Cl}_{2}$ a,b,c

\begin{tabular}{|c|c|c|c|c|}
\hline atom & $x$ & $y$ & $z$ & $B_{\mathrm{eq}}$ \\
\hline$\overline{\operatorname{Pt}(1)}$ & $0.26081(3)$ & $0.26228(2)$ & $0.06920(2)$ & $1.849(8)$ \\
\hline $\operatorname{Pt}(2)$ & $0.25203(3)$ & $0.22878(2)$ & $-0.07655(2)$ & $2.055(9)$ \\
\hline $\operatorname{Pt}(3)$ & $0.23614(4)$ & $0.21625(2)$ & $-0.21190(2)$ & $2.692(10)$ \\
\hline $\mathrm{Cl}(1)$ & $0.7759(4)$ & $0.0256(3)$ & $0.7244(3)$ & $8.0(2)$ \\
\hline $\mathrm{Cl}(2)$ & $0.6152(6)$ & $-0.0872(4)$ & $0.7323(4)$ & $11.4(2)$ \\
\hline $\mathrm{Cl}(3)$ & $0.5749(5)$ & $0.1318(3)$ & $0.7027(3)$ & $8.4(2)$ \\
\hline $\mathrm{Cl}(4)$ & $0.5693(6)$ & $0.0403(4)$ & $0.5777(4)$ & $11.0(2)$ \\
\hline $\mathrm{Cl}(5)$ & $0.7808(7)$ & $0.5026(5)$ & $0.4189(5)$ & $12.3(3)$ \\
\hline $\mathrm{Cl}(6)$ & $0.6169(6)$ & $0.4142(3)$ & $0.4541(3)$ & $10.4(2)$ \\
\hline $\mathrm{Cl}(7)$ & $0.4808(5)$ & $0.2016(5)$ & $0.4727(5)$ & 11.4(3) \\
\hline $\mathrm{Cl}(8)$ & $0.5920(7)$ & $0.2044(5)$ & $0.3608(4)$ & $12.3(3)$ \\
\hline $\mathrm{Cl}(9)$ & $0.7601(9)$ & $0.0864(6)$ & $0.4431(10)$ & $22.3(6)$ \\
\hline $\mathrm{Cl}(10)$ & $0.923(2)$ & $0.1720(7)$ & $0.424(1)$ & 29.9(9) \\
\hline $\mathrm{P}(1)$ & $0.0984(2)$ & $0.2259(1)$ & $0.0606(2)$ & $1.99(5)$ \\
\hline $\mathrm{P}(2)$ & $0.0975(2)$ & $0.1755(1)$ & $-0.0885(1)$ & $2.19(5)$ \\
\hline$P(3)$ & $0.1511(2)$ & $0.1024(2)$ & $-0.2130(2)$ & $2.59(6)$ \\
\hline $\mathrm{P}(4)$ & $0.4242(2)$ & $0.2918(1)$ & $0.0757(1)$ & $1.92(5)$ \\
\hline$P(5)$ & $0.4008(2)$ & $0.2911(1)$ & $-0.0777(1)$ & $2.03(5)$ \\
\hline$P(6)$ & $0.3153(2)$ & $0.3316(2)$ & $-0.2096(2)$ & $2.52(6)$ \\
\hline $\mathrm{P}(11)$ & $0.6958(2)$ & $0.4856(2)$ & $0.9756(2)$ & $3.49(7)$ \\
\hline$P(12)$ & $0.7984(2)$ & $0.0211(2)$ & $0.9768(2)$ & $2.75(6)$ \\
\hline$P(13)$ & $0.8092(4)$ & $0.3103(3)$ & $0.6065(4)$ & $6.9(2)$ \\
\hline $\mathrm{F}(11)$ & $0.6184(7)$ & $0.5052(5)$ & $1.0225(6)$ & $5.9(3)$ \\
\hline$F(12)$ & $0.6117(6)$ & $0.4515(4)$ & $0.9166(5)$ & $5.0(2)$ \\
\hline $\mathrm{F}(13)$ & $0.7710(6)$ & $0.4684(5)$ & $0.9274(5)$ & $4.9(2)$ \\
\hline $\mathrm{F}(14)$ & $0.7778(6)$ & $0.5227(4)$ & $1.0344(5)$ & $5.4(2)$ \\
\hline $\mathrm{F}(15)$ & $0.6987(6)$ & $0.5604(4)$ & $0.9449(6)$ & $5.8(2)$ \\
\hline $\mathrm{F}(16)$ & $0.6919(5)$ & $0.4118(4)$ & $1.0063(5)$ & $4.2(2)$ \\
\hline $\mathrm{F}(21)$ & $0.7428(6)$ & $-0.0067(5)$ & $1.0360(5)$ & $5.2(2)$ \\
\hline $\mathrm{F}(22)$ & $0.7028(6)$ & $0.0304(5)$ & $0.9375(5)$ & $5.4(2)$ \\
\hline $\mathrm{F}(23)$ & $0.7811(6)$ & $-0.0592(4)$ & $0.9410(5)$ & $5.5(2)$ \\
\hline $\mathrm{F}(24)$ & $0.8548(6)$ & $0.0467(5)$ & $0.9160(5)$ & $5.9(2)$ \\
\hline$F(25)$ & $0.8957(5)$ & $0.0118(4)$ & $1.0163(5)$ & $4.6(2)$ \\
\hline$F(26)$ & $0.8164(6)$ & $0.0994(4)$ & $1.0122(6)$ & $5.9(2)$ \\
\hline $\mathrm{F}(31)$ & $0.718(1)$ & $0.329(1)$ & $0.5753(10)$ & $16.9(8)$ \\
\hline $\mathrm{F}(32)$ & $0.759(1)$ & $0.285(1)$ & $0.6709(8)$ & $11.5(5)$ \\
\hline$F(33)$ & $0.845(1)$ & $0.3851(9)$ & $0.643(1)$ & $16.6(8)$ \\
\hline $\mathrm{F}(34)$ & $0.904(1)$ & $0.2889(8)$ & $0.636(1)$ & $12.0(6)$ \\
\hline $\mathrm{F}(35)$ & $0.854(1)$ & $0.330(1)$ & $0.543(1)$ & $15.2(8)$ \\
\hline$F(36)$ & $0.781(2)$ & $0.2312(9)$ & $0.574(1)$ & $14.2(7)$ \\
\hline $\mathrm{N}(1)$ & $0.2717(7)$ & $0.3502(5)$ & $0.2112(6)$ & $3.2(2)$ \\
\hline $\mathrm{N}(2)$ & $0.2246(6)$ & $0.1953(5)$ & $-0.3654(5)$ & $2.2(2)$ \\
\hline$C(1)$ & $0.2627(8)$ & $0.3173(6)$ & $0.1594(6)$ & $2.6(2)$ \\
\hline$C(2)$ & $0.228(1)$ & $0.2085(8)$ & $-0.3084(9)$ & $4.6(3)$ \\
\hline$C(3)$ & $0.0531(8)$ & $0.1537(6)$ & $-0.0080(6)$ & $2.4(2)$ \\
\hline$C(4)$ & $0.0782(8)$ & $0.0910(6)$ & $-0.1415(6)$ & $2.5(2)$ \\
\hline$C(5)$ & $0.4572(8)$ & $0.3419(6)$ & $0.0044(6)$ & $2.3(2)$ \\
\hline$C(6)$ & $0.3983(8)$ & $0.3620(6)$ & $-0.1320(6)$ & $2.6(2)$ \\
\hline$C(11)$ & $0.293(1)$ & $0.3781(7)$ & $0.2814(8)$ & $3.8(3)$ \\
\hline$C(12)$ & $0.293(1)$ & $0.4508(9)$ & $0.2973(9)$ & $5.0(3)$ \\
\hline$C(13)$ & $0.316(1)$ & $0.4753(10)$ & $0.3640(10)$ & $5.7(4)$ \\
\hline$C(14)$ & $0.344(1)$ & $0.433(1)$ & $0.412(1)$ & $6.7(4)$ \\
\hline$C(15)$ & $0.346(1)$ & $0.366(1)$ & $0.395(1)$ & $6.3(4)$ \\
\hline
\end{tabular}




\begin{tabular}{|c|c|c|c|c|}
\hline$C(16)$ & $0.318(1)$ & $0.3342(8)$ & $0.3285(8)$ & $4.4(3)$ \\
\hline$C(17)$ & $0.266(2)$ & $0.497(1)$ & $0.242(1)$ & 7.1(5) \\
\hline$C(18)$ & $0.313(1)$ & $0.2582(9)$ & $0.3076(9)$ & $5.3(3)$ \\
\hline$C(21)$ & $0.216(1)$ & $0.1772(9)$ & $-0.4429(9)$ & $4.9(3)$ \\
\hline$C(22)$ & $0.165(1)$ & $0.215(1)$ & $-0.484(1)$ & $6.4(4)$ \\
\hline$C(23)$ & $0.159(2)$ & $0.196(1)$ & $-0.556(1)$ & $7.2(5)$ \\
\hline$C(24)$ & $0.202(1)$ & $0.1420(10)$ & $-0.5814(10)$ & $5.4(4)$ \\
\hline$C(25)$ & $0.250(1)$ & $0.102(1)$ & $-0.542(1)$ & $6.7(4)$ \\
\hline$C(26)$ & $0.260(1)$ & $0.1246(10)$ & $-0.466(1)$ & $5.9(4)$ \\
\hline$C(27)$ & $0.122(2)$ & $0.273(1)$ & $-0.453(1)$ & $9.9(7)$ \\
\hline$C(28)$ & $0.321(2)$ & $0.092(1)$ & $-0.420(1)$ & $9.3(7)$ \\
\hline$C(111)$ & $0.0639(8)$ & $0.1925(6)$ & $0.1398(6)$ & $2.3(2)$ \\
\hline$C(112)$ & $0.0311(9)$ & $0.2341(7)$ & $0.1878(7)$ & $3.2(2)$ \\
\hline$C(113)$ & $0.015(1)$ & $0.2105(8)$ & $0.2524(8)$ & $4.3(3)$ \\
\hline$C(114)$ & $0.032(1)$ & $0.1446(8)$ & $0.2651(8)$ & $4.4(3)$ \\
\hline$C(115)$ & $0.068(1)$ & $0.1019(8)$ & $0.2178(8)$ & $4.0(3)$ \\
\hline$C(116)$ & $0.0833(9)$ & $0.1254(6)$ & $0.1543(6)$ & $2.9(2)$ \\
\hline$C(121)$ & $0.0290(8)$ & $0.2935(6)$ & $0.0499(6)$ & $2.7(2)$ \\
\hline$C(122)$ & $0.0751(9)$ & $0.3652(7)$ & $0.0573(7)$ & $3.4(2)$ \\
\hline$C(123)$ & $0.023(1)$ & $0.4177(8)$ & $0.0529(8)$ & $4.3(3)$ \\
\hline$C(124)$ & $-0.077(1)$ & 0.3991(8) & $0.0381(8)$ & $4.3(3)$ \\
\hline$C(125)$ & $-0.122(1)$ & $0.3288(8)$ & $0.0277(8)$ & $4.3(3)$ \\
\hline$C(126)$ & $-0.0688(9)$ & $0.2761(7)$ & $0.0349(7)$ & $3.4(2)$ \\
\hline$C(211)$ & $0.0168(9)$ & $0.2285(6)$ & $-0.1273(7)$ & $3.1(2)$ \\
\hline$C(212)$ & $0.049(1)$ & $0.3033(8)$ & $-0.1184(8)$ & $4.2(3)$ \\
\hline$C(213)$ & $-0.014(1)$ & $0.3467(8)$ & $-0.1421(8)$ & $4.7(3)$ \\
\hline$C(214)$ & $-0.100(1)$ & $0.314(1)$ & $-0.179(1)$ & $5.9(4)$ \\
\hline$C(215)$ & $-0.133(1)$ & $0.2398(9)$ & $-0.1884(9)$ & $5.5(4)$ \\
\hline$C(216)$ & $-0.072(1)$ & $0.1991(7)$ & $-0.1580(8)$ & $4.0(3)$ \\
\hline$C(311)$ & $0.0659(8)$ & $0.0785(6)$ & $-0.2914(6)$ & $2.6(2)$ \\
\hline$C(312)$ & $0.0092(10)$ & $0.1248(7)$ & $-0.3078(7)$ & $3.7(2)$ \\
\hline$C(313)$ & $-0.054(1)$ & $0.1126(8)$ & $-0.3683(8)$ & $4.3(3)$ \\
\hline$C(314)$ & $-0.055(1)$ & $0.0523(8)$ & $-0.4115(8)$ & $4.7(3)$ \\
\hline$C(315)$ & $0.003(1)$ & $0.0061(8)$ & $-0.3972(9)$ & $4.8(3)$ \\
\hline$C(316)$ & $0.0660(10)$ & $0.0178(7)$ & $-0.3354(7)$ & $3.5(2)$ \\
\hline$C(321)$ & $0.2228(9)$ & $0.0341(6)$ & $-0.2149(6)$ & $3.0(2)$ \\
\hline$C(322)$ & $0.192(1)$ & $-0.0332(8)$ & $-0.1924(8)$ & $4.0(3)$ \\
\hline$C(323)$ & $0.244(1)$ & $-0.0827(8)$ & $-0.1978(8)$ & $4.4(3)$ \\
\hline$C(324)$ & $0.329(1)$ & $-0.0702(9)$ & $-0.2243(9)$ & $5.3(3)$ \\
\hline$C(325)$ & $0.364(1)$ & $-0.0052(8)$ & $-0.2434(8)$ & $4.7(3)$ \\
\hline$C(326)$ & $0.3113(10)$ & $0.0471(7)$ & $-0.2376(7)$ & $3.7(2)$ \\
\hline$C(411)$ & $0.4814(8)$ & $0.3485(6)$ & $0.1538(6)$ & $2.3(2)$ \\
\hline$C(412)$ & $0.5183(10)$ & $0.3206(7)$ & $0.2092(7)$ & $3.8(3)$ \\
\hline$C(413)$ & $0.550(1)$ & $0.3605(8)$ & $0.2717(8)$ & $4.6(3)$ \\
\hline$C(414)$ & $0.546(1)$ & $0.4331(8)$ & $0.2760(8)$ & $4.3(3)$ \\
\hline$C(415)$ & $0.515(1)$ & $0.4623(8)$ & $0.2213(8)$ & $4.1(3)$ \\
\hline$C(416)$ & $0.4801(9)$ & $0.4212(7)$ & $0.1601(7)$ & $3.5(2)$ \\
\hline$C(421)$ & $0.4860(7)$ & $0.2191(5)$ & $0.0706(5)$ & $1.9(2)$ \\
\hline$C(422)$ & $0.4368(9)$ & $0.1496(6)$ & $0.0715(6)$ & $2.9(2)$ \\
\hline$C(423)$ & $0.4864(9)$ & $0.0933(6)$ & $0.0674(6)$ & $3.0(2)$ \\
\hline$C(424)$ & $0.5835(9)$ & $0.1054(7)$ & $0.0624(7)$ & $3.4(2)$ \\
\hline$C(425)$ & $0.6301(9)$ & $0.1746(7)$ & $0.0616(7)$ & $3.4(2)$ \\
\hline$C(426)$ & $0.5827(8)$ & $0.2306(6)$ & $0.0656(6)$ & $2.6(2)$ \\
\hline$C(511)$ & $0.4894(8)$ & $0.2422(6)$ & $-0.1023(6)$ & $2.5(2)$ \\
\hline$C(512)$ & $0.4682(8)$ & $0.1672(6)$ & $-0.1080(6)$ & $2.8(2)$ \\
\hline$C(513)$ & $0.5386(9)$ & $0.1285(7)$ & $-0.1207(7)$ & $3.2(2)$ \\
\hline$C(514)$ & $0.630(1)$ & $0.1652(8)$ & $-0.1251(8)$ & $4.2(3)$ \\
\hline$C(515)$ & $0.650(1)$ & $0.2383(7)$ & $-0.1208(7)$ & $3.9(3)$ \\
\hline
\end{tabular}




\begin{tabular}{|c|c|c|c|c|}
\hline$C(516)$ & $0.5816(9)$ & $0.2768(7)$ & $-0.1090(7)$ & $3.2(2)$ \\
\hline$C(611)$ & $0.2377(9)$ & $0.3930(7)$ & $-0.2215(7)$ & $3.4(2)$ \\
\hline$C(612)$ & $0.150(1)$ & $0.3717(8)$ & $-0.2648(8)$ & $4.6(3)$ \\
\hline$C(613)$ & $0.091(1)$ & $0.423(1)$ & $-0.275(1)$ & $6.0(4)$ \\
\hline$C(614)$ & $0.116(1)$ & $0.486(1)$ & $-0.243(1)$ & $6.3(4)$ \\
\hline$C(615)$ & $0.194(1)$ & $0.5097(9)$ & $-0.1995(10)$ & $5.5(4)$ \\
\hline$C(616)$ & $0.259(1)$ & $0.4623(9)$ & $-0.1879(9)$ & $4.8(3)$ \\
\hline$C(621)$ & $0.3880(9)$ & $0.3412(6)$ & $-0.2794(6)$ & $3.0(2)$ \\
\hline$C(622)$ & $0.3663(10)$ & $0.3779(7)$ & $-0.3338(7)$ & $3.6(2)$ \\
\hline$C(623)$ & $0.421(1)$ & $0.3818(9)$ & $-0.3875(9)$ & $5.5(4)$ \\
\hline$C(624)$ & $0.502(1)$ & $0.3508(9)$ & $-0.3850(9)$ & $5.0(3)$ \\
\hline$C(625)$ & $0.525(1)$ & $0.3117(9)$ & $-0.3320(9)$ & $5.1(3)$ \\
\hline$C(626)$ & $0.468(1)$ & $0.3071(8)$ & $-0.2791(8)$ & $4.4(3)$ \\
\hline$C(701)$ & $0.690(2)$ & $-0.014(1)$ & $0.777(1)$ & $7.9(5)$ \\
\hline$C(702)$ & $0.625(2)$ & $0.123(1)$ & $0.626(1)$ & $9.0(6)$ \\
\hline$C(703)$ & $0.749(2)$ & $0.437(2)$ & $0.466(2)$ & $10.9(8)$ \\
\hline$C(704)$ & $0.585(3)$ & $0.200(2)$ & $0.449(2)$ & $15(1)$ \\
\hline$C(705)$ & $0.821(3)$ & $0.167(2)$ & $0.433(2)$ & $14(1)$ \\
\hline $\mathrm{H}(1)$ & 0.0723 & 0.1115 & 0.0049 & 3.0 \\
\hline $\mathrm{H}(2)$ & -0.0146 & 0.1459 & -0.0135 & 3.0 \\
\hline $\mathrm{H}(3)$ & 0.0972 & 0.0554 & -0.1137 & 3.1 \\
\hline $\mathrm{H}(4)$ & 0.0129 & 0.0755 & -0.1575 & 3.1 \\
\hline $\mathrm{H}(5)$ & 0.4392 & 0.3866 & 0.0093 & 2.8 \\
\hline $\mathrm{H}(6)$ & 0.5254 & 0.3496 & 0.0046 & 2.8 \\
\hline $\mathrm{H}(7)$ & 0.3792 & 0.4008 & -0.1072 & 2.9 \\
\hline $\mathrm{H}(8)$ & 0.4598 & 0.3773 & -0.1446 & 2.9 \\
\hline $\mathrm{H}(21)$ & 0.3142 & 0.5362 & 0.2394 & 8.3 \\
\hline $\mathrm{H}(22)$ & 0.2473 & 0.4706 & 0.1981 & 8.3 \\
\hline $\mathrm{H}(23)$ & 0.2097 & 0.5169 & 0.2537 & 8.3 \\
\hline $\mathrm{H}(24)$ & 0.2473 & 0.2356 & 0.2890 & 6.1 \\
\hline $\mathrm{H}(25)$ & 0.3512 & 0.2510 & 0.2728 & 6.1 \\
\hline $\mathrm{H}(26)$ & 0.3293 & 0.2350 & 0.3459 & 6.1 \\
\hline $\mathrm{H}(27)$ & 0.1675 & 0.3113 & -0.4236 & 11.2 \\
\hline $\mathrm{H}(28)$ & 0.0902 & 0.2968 & -0.4864 & 11.2 \\
\hline $\mathrm{H}(29)$ & 0.0751 & 0.2564 & -0.4227 & 11.2 \\
\hline $\mathrm{H}(30)$ & 0.2857 & 0.0648 & -0.3857 & 16.3 \\
\hline $\mathrm{H}(31)$ & 0.3498 & 0.0534 & -0.4427 & 16.3 \\
\hline $\mathrm{H}(32)$ & 0.3742 & 0.1219 & -0.3912 & 16.3 \\
\hline $\mathrm{H}(33)$ & 0.0174 & 0.2795 & 0.1775 & 3.9 \\
\hline $\mathrm{H}(34)$ & -0.0114 & 0.2386 & 0.2853 & 5.8 \\
\hline $\mathrm{H}(35)$ & 0.0164 & 0.1265 & 0.3077 & 5.6 \\
\hline $\mathrm{H}(36)$ & 0.0825 & 0.0559 & 0.2275 & 4.8 \\
\hline $\mathrm{H}(37)$ & 0.1073 & 0.0961 & 0.1209 & 3.8 \\
\hline $\mathrm{H}(38)$ & 0.1424 & 0.3774 & 0.0631 & 4.4 \\
\hline $\mathrm{H}(39)$ & 0.0530 & 0.4670 & 0.0603 & 5.2 \\
\hline $\mathrm{H}(40)$ & -0.1118 & 0.4364 & 0.0323 & 5.6 \\
\hline $\mathrm{H}(41)$ & -0.1900 & 0.3184 & 0.0184 & 5.6 \\
\hline $\mathrm{H}(42)$ & -0.1005 & 0.2267 & 0.0260 & 4.2 \\
\hline $\mathrm{H}(43)$ & 0.1115 & 0.3252 & -0.0931 & 4.9 \\
\hline $\mathrm{H}(44)$ & 0.0051 & 0.3973 & -0.1319 & 6.7 \\
\hline $\mathrm{H}(45)$ & -0.1406 & 0.3458 & -0.1970 & 7.0 \\
\hline $\mathrm{H}(46)$ & -0.1928 & 0.2193 & -0.2151 & 7.3 \\
\hline $\mathrm{H}(47)$ & -0.0938 & 0.1471 & -0.1596 & 5.7 \\
\hline $\mathrm{H}(48)$ & 0.5194 & 0.2704 & 0.2058 & 4.3 \\
\hline $\mathrm{H}(49)$ & 0.5785 & 0.3402 & 0.3103 & 5.6 \\
\hline $\mathrm{H}(50)$ & 0.5685 & 0.4626 & 0.3198 & 5.1 \\
\hline $\mathrm{H}(51)$ & 0.5091 & 0.5118 & 0.2238 & 5.4 \\
\hline $\mathrm{H}(52)$ & 0.4564 & 0.4430 & 0.1217 & 3.9 \\
\hline
\end{tabular}




\begin{tabular}{|c|c|c|c|c|}
\hline $\mathrm{H}(53)$ & 0.3707 & 0.1411 & 0.0752 & 3.4 \\
\hline $\mathrm{H}(54)$ & 0.4515 & 0.0446 & 0.0676 & 3.9 \\
\hline $\mathrm{H}(55)$ & 0.6164 & 0.0660 & 0.0594 & 4.1 \\
\hline $\mathrm{H}(56)$ & 0.6957 & 0.1835 & 0.0553 & 4.1 \\
\hline $\mathrm{H}(57)$ & 0.6158 & 0.2783 & 0.0623 & 3.1 \\
\hline $\mathrm{H}(58)$ & 0.4049 & 0.1433 & -0.1032 & 3.3 \\
\hline $\mathrm{H}(59)$ & 0.5239 & 0.0775 & -0.1259 & 3.9 \\
\hline $\mathrm{H}(60)$ & 0.6803 & 0.1375 & -0.1289 & 4.8 \\
\hline $\mathrm{H}(61)$ & 0.7154 & 0.2627 & -0.1242 & 5.0 \\
\hline $\mathrm{H}(62)$ & 0.5947 & 0.3283 & -0.1088 & 4.0 \\
\hline $\mathrm{H}(63)$ & 0.1336 & 0.3230 & -0.2874 & 6.4 \\
\hline $\mathrm{H}(64)$ & 0.0369 & 0.4067 & -0.3124 & 9.6 \\
\hline $\mathrm{H}(65)$ & 0.0737 & 0.5209 & -0.2507 & 7.4 \\
\hline $\mathrm{H}(66)$ & 0.2099 & 0.5577 & -0.1771 & 7.2 \\
\hline $\mathrm{H}(67)$ & 0.3164 & 0.4776 & -0.1584 & 5.1 \\
\hline $\mathrm{H}(68)$ & 0.0107 & 0.1671 & -0.2777 & 4.6 \\
\hline $\mathrm{H}(69)$ & -0.0973 & 0.1447 & -0.3793 & 5.5 \\
\hline $\mathrm{H}(70)$ & -0.0972 & 0.0447 & -0.4550 & 5.3 \\
\hline $\mathrm{H}(71)$ & 0.0032 & -0.0359 & -0.4280 & 6.2 \\
\hline $\mathrm{H}(72)$ & 0.1078 & -0.0137 & -0.3265 & 4.1 \\
\hline $\mathrm{H}(73)$ & 0.3141 & 0.4010 & -0.3333 & 4.6 \\
\hline $\mathrm{H}(74)$ & 0.3992 & 0.4058 & -0.4265 & 7.2 \\
\hline $\mathrm{H}(75)$ & 0.5454 & 0.3566 & -0.4203 & 6.0 \\
\hline $\mathrm{H}(76)$ & 0.5796 & 0.2881 & -0.3314 & 6.9 \\
\hline $\mathrm{H}(77)$ & 0.4823 & 0.2808 & -0.2426 & 5.4 \\
\hline $\mathrm{H}(78)$ & 0.1337 & -0.0431 & -0.1710 & 4.7 \\
\hline $\mathrm{H}(79)$ & 0.2208 & -0.1285 & -0.1867 & 5.3 \\
\hline $\mathrm{H}(80)$ & 0.3634 & -0.1087 & -0.2280 & 6.4 \\
\hline $\mathrm{H}(81)$ & 0.4251 & 0.0039 & -0.2608 & 5.5 \\
\hline $\mathrm{H}(82)$ & 0.3333 & 0.0942 & -0.2504 & 4.5 \\
\hline $\mathrm{H}(83)$ & 0.2680 & 0.2049 & 0.0040 & 3.1 \\
\hline $\mathrm{H}(84)$ & 0.3127 & 0.5229 & 0.3786 & 6.8 \\
\hline $\mathrm{H}(85)$ & 0.3615 & 0.4533 & 0.4579 & 8.1 \\
\hline $\mathrm{H}(86)$ & 0.3657 & 0.3387 & 0.4299 & 7.6 \\
\hline $\mathrm{H}(87)$ & 0.1250 & 0.2203 & -0.5868 & 8.6 \\
\hline $\mathrm{H}(88)$ & 0.1994 & 0.1320 & -0.6298 & 6.4 \\
\hline $\mathrm{H}(89)$ & 0.2743 & 0.0636 & -0.5606 & 7.9 \\
\hline
\end{tabular}

a Estimated standard deviations are given in parentheses. $\mathrm{b}$ The $\mathrm{Pt}, \mathrm{Cl}, \mathrm{P}$ and $\mathrm{F}$ atoms were assigned with anisotropic thermal parameters given as the isotropic equivalent displacement parameter defined as $B_{\text {eq }}=\left(8 \pi^{2} / 3\right)\left(U_{11}\left(a a^{*}\right)^{2}+U_{22}\left(b b^{*}\right)^{2}+U_{33}\left(c c^{*}\right)^{2}+2 U_{12} a a^{*} b b^{*} \cos \gamma+\right.$ $\left.2 U_{13} a a^{*} c c^{*} \cos \beta+2 U_{23} b b^{*} c c^{*} \cos \alpha\right)$. Other non-hydrogen atoms were refined isotropically. ${ }^{c}$ All hydrogen atoms except for solvents were calculated with $\mathrm{C}-\mathrm{H}=0.95 \AA$, were not refined with the appropriate $B$ iso.

Table S7-3. Final Anisotropic Thermal Parameters for $9 \mathbf{b} \cdot 5 \mathrm{CH}_{2} \mathrm{Cl}_{2}{ }^{\mathrm{a}, \mathrm{b}}$

\begin{tabular}{lllcrrr}
\hline atom & $U_{11}$ & $U_{22}$ & $U_{33}$ & $U_{12}$ & $U_{13}$ & $U_{23}$ \\
\hline $\operatorname{Pt}(1)$ & $0.0191(2)$ & $0.0229(2)$ & $0.0282(2)$ & $0.0045(1)$ & $0.0012(2)$ & $0.0030(2)$ \\
$\operatorname{Pt}(2)$ & $0.0234(2)$ & $0.0263(2)$ & $0.0275(2)$ & $0.0005(2)$ & $0.0030(2)$ & $0.0063(2)$ \\
$\operatorname{Pt}(3)$ & $0.0387(3)$ & $0.0345(2)$ & $0.0252(2)$ & $-0.0039(2)$ & $0.0000(2)$ & $0.0085(2)$ \\
$\mathrm{Cl}(1)$ & $0.083(4)$ & $0.128(5)$ & $0.094(4)$ & $0.017(3)$ & $0.006(3)$ & $0.033(4)$ \\
$\mathrm{Cl}(2)$ & $0.179(7)$ & $0.094(4)$ & $0.145(7)$ & $-0.017(5)$ & $0.050(6)$ & $-0.020(4)$ \\
$\mathrm{Cl}(3)$ & $0.141(5)$ & $0.111(4)$ & $0.071(3)$ & $0.029(4)$ & $0.015(3)$ & $0.025(3)$ \\
$\mathrm{Cl}(4)$ & $0.137(6)$ & $0.149(6)$ & $0.122(6)$ & $0.050(5)$ & $-0.031(5)$ & $-0.040(5)$ \\
$\mathrm{Cl}(5)$ & $0.181(8)$ & $0.174(8)$ & $0.152(7)$ & $0.088(6)$ & $0.040(6)$ & $0.089(6)$
\end{tabular}




\begin{tabular}{|c|c|c|c|c|c|c|}
\hline $\mathrm{Cl}(6)$ & $0.236(9)$ & $0.102(4)$ & $0.043(3)$ & $0.012(5)$ & $-0.005(4)$ & $0.004(3)$ \\
\hline $\mathrm{Cl}(7)$ & $0.109(5)$ & $0.179(8)$ & $0.155(7)$ & $0.031(5)$ & $0.025(5)$ & $0.042(6)$ \\
\hline $\mathrm{Cl}(8)$ & $0.235(10)$ & $0.149(7)$ & $0.098(5)$ & $0.070(7)$ & $0.034(6)$ & $0.020(5)$ \\
\hline $\mathrm{Cl}(9)$ & $0.20(1)$ & $0.127(8)$ & $0.48(3)$ & $0.010(8)$ & $-0.09(1)$ & $0.01(1)$ \\
\hline $\mathrm{Cl}(10)$ & $0.63(3)$ & $0.18(1)$ & $0.42(2)$ & $0.13(2)$ & $0.42(3)$ & $0.04(1)$ \\
\hline$P(1)$ & $0.019(1)$ & $0.023(1)$ & $0.035(1)$ & $0.0057(10)$ & $0.005(1)$ & $0.005(1)$ \\
\hline $\mathrm{P}(2)$ & $0.025(1)$ & $0.028(1)$ & $0.028(1)$ & $-0.002(1)$ & $0.004(1)$ & $0.005(1)$ \\
\hline $\mathrm{P}(3)$ & $0.038(2)$ & $0.031(1)$ & $0.026(1)$ & $-0.001(1)$ & $0.005(1)$ & $0.003(1)$ \\
\hline $\mathrm{P}(4)$ & $0.016(1)$ & $0.025(1)$ & $0.031(1)$ & $0.0043(10)$ & $0.002(1)$ & $0.001(1)$ \\
\hline$P(5)$ & $0.025(1)$ & $0.019(1)$ & $0.033(1)$ & $0.0018(10)$ & $0.006(1)$ & $0.003(1)$ \\
\hline$P(6)$ & $0.035(2)$ & $0.030(1)$ & $0.033(2)$ & $0.008(1)$ & $0.005(1)$ & $0.012(1)$ \\
\hline $\mathrm{P}(11)$ & $0.028(2)$ & $0.031(2)$ & $0.072(3)$ & $0.008(1)$ & $0.001(2)$ & $0.000(2)$ \\
\hline$P(12)$ & $0.032(2)$ & $0.028(1)$ & $0.046(2)$ & $0.008(1)$ & $0.006(1)$ & $0.004(1)$ \\
\hline$P(13)$ & $0.070(3)$ & $0.089(4)$ & $0.109(5)$ & $0.024(3)$ & $0.009(3)$ & $0.028(3)$ \\
\hline $\mathrm{F}(11)$ & $0.069(6)$ & $0.053(5)$ & $0.111(8)$ & $0.022(4)$ & $0.038(6)$ & $0.002(5)$ \\
\hline $\mathrm{F}(12)$ & $0.048(5)$ & $0.042(4)$ & $0.090(7)$ & $-0.004(4)$ & $-0.019(5)$ & $0.009(4)$ \\
\hline $\mathrm{F}(13)$ & $0.056(5)$ & $0.069(6)$ & $0.065(6)$ & $0.021(4)$ & $0.018(4)$ & $0.010(5)$ \\
\hline $\mathrm{F}(14)$ & $0.052(5)$ & $0.054(5)$ & $0.087(7)$ & $-0.006(4)$ & $0.003(5)$ & $-0.017(5)$ \\
\hline $\mathrm{F}(15)$ & $0.045(5)$ & $0.038(4)$ & $0.133(9)$ & $0.002(4)$ & $-0.012(5)$ & $0.026(5)$ \\
\hline$F(16)$ & $0.041(4)$ & $0.035(4)$ & $0.084(6)$ & $0.009(3)$ & $-0.003(4)$ & $0.013(4)$ \\
\hline $\mathrm{F}(21)$ & $0.053(5)$ & $0.079(6)$ & $0.067(6)$ & $0.008(4)$ & $0.022(5)$ & $0.013(5)$ \\
\hline $\mathrm{F}(22)$ & $0.046(5)$ & $0.069(6)$ & $0.090(7)$ & $0.006(4)$ & $-0.013(5)$ & $0.031(5)$ \\
\hline$F(23)$ & $0.051(5)$ & $0.041(4)$ & $0.111(8)$ & $0.000(4)$ & $0.024(5)$ & $-0.015(5)$ \\
\hline $\mathrm{F}(24)$ & $0.054(5)$ & $0.099(7)$ & $0.059(6)$ & $-0.025(5)$ & $0.009(4)$ & $0.025(5)$ \\
\hline $\mathrm{F}(25)$ & $0.042(4)$ & $0.056(5)$ & $0.078(6)$ & $0.014(4)$ & $-0.007(4)$ & $0.018(4)$ \\
\hline$F(26)$ & $0.054(5)$ & $0.040(4)$ & $0.121(9)$ & $0.003(4)$ & $0.010(5)$ & $-0.021(5)$ \\
\hline $\mathrm{F}(31)$ & $0.10(1)$ & $0.43(3)$ & $0.16(2)$ & $0.11(2)$ & $0.03(1)$ & $0.17(2)$ \\
\hline $\mathrm{F}(32)$ & $0.13(1)$ & $0.22(2)$ & $0.10(1)$ & $0.05(1)$ & $0.036(9)$ & $0.07(1)$ \\
\hline$F(33)$ & $0.16(2)$ & $0.10(1)$ & $0.35(3)$ & $0.02(1)$ & $0.00(2)$ & $-0.06(2)$ \\
\hline $\mathrm{F}(34)$ & $0.10(1)$ & $0.13(1)$ & $0.23(2)$ & $0.031(9)$ & $-0.02(1)$ & $0.06(1)$ \\
\hline$F(35)$ & $0.18(2)$ & $0.20(2)$ & $0.27(2)$ & $0.08(1)$ & $0.12(2)$ & $0.17(2)$ \\
\hline$F(36)$ & $0.23(2)$ & $0.12(1)$ & $0.18(2)$ & $-0.02(1)$ & $0.03(2)$ & $0.00(1)$ \\
\hline
\end{tabular}

$\mathrm{a}$ Estmated standard deviations are given in parentheses. $\mathrm{b}$ Parameters have the form $\exp (-$ $\left.2 \pi^{2}\left(U_{11} h^{2} a^{* 2}+\cdots+2 U_{12} h k a^{*} b^{*} \cos \gamma+\cdots\right)\right)$

Table S7-4. Bond Lengths for Non-hydrogen Atoms of $9 b \cdot 5 \mathrm{CH}_{2} \mathrm{Cl}_{2}{ }^{\mathrm{a}}$

\begin{tabular}{llcllc}
\hline atom & atom & distance & atom & atom & distance \\
\hline $\operatorname{Pt}(1)$ & $\mathrm{Pt}(2)$ & $2.8680(6)$ & $\mathrm{P}(3)$ & $\mathrm{C}(4)$ & $1.85(1)$ \\
$\mathrm{Pt}(1)$ & $\mathrm{P}(1)$ & $2.313(3)$ & $\mathrm{P}(3)$ & $\mathrm{C}(311)$ & $1.84(1)$ \\
$\mathrm{Pt}(1)$ & $\mathrm{P}(4)$ & $2.318(3)$ & $\mathrm{P}(3)$ & $\mathrm{C}(321)$ & $1.82(1)$ \\
$\mathrm{Pt}(1)$ & $\mathrm{C}(1)$ & $1.98(1)$ & $\mathrm{P}(4)$ & $\mathrm{C}(5)$ & $1.83(1)$ \\
$\mathrm{Pt}(2)$ & $\mathrm{Pt}(3)$ & $2.6374(6)$ & $\mathrm{P}(4)$ & $\mathrm{C}(411)$ & $1.83(1)$ \\
$\mathrm{Pt}(2)$ & $\mathrm{P}(2)$ & $2.265(3)$ & $\mathrm{P}(4)$ & $\mathrm{C}(421)$ & $1.79(1)$ \\
$\mathrm{Pt}(2)$ & $\mathrm{P}(5)$ & $2.272(3)$ & $\mathrm{P}(5)$ & $\mathrm{C}(5)$ & $1.84(1)$ \\
$\mathrm{Pt}(3)$ & $\mathrm{P}(3)$ & $2.313(3)$ & $\mathrm{P}(5)$ & $\mathrm{C}(6)$ & $1.82(1)$ \\
$\mathrm{Pt}(3)$ & $\mathrm{P}(6)$ & $2.305(3)$ & $\mathrm{P}(5)$ & $\mathrm{C}(511)$ & $1.80(1)$ \\
$\mathrm{Pt}(3)$ & $\mathrm{C}(2)$ & $1.88(2)$ & $\mathrm{P}(6)$ & $\mathrm{C}(6)$ & $1.83(1)$ \\
$\mathrm{Cl}(1)$ & $\mathrm{C}(701)$ & $1.81(2)$ & $\mathrm{P}(6)$ & $\mathrm{C}(611)$ & $1.79(1)$ \\
$\mathrm{Cl}(2)$ & $\mathrm{C}(701)$ & $1.72(2)$ & $\mathrm{P}(6)$ & $\mathrm{C}(621)$ & $1.82(1)$ \\
$\mathrm{Cl}(3)$ & $\mathrm{C}(702)$ & $1.75(3)$ & $\mathrm{P}(11)$ & $\mathrm{F}(11)$ & $1.595(9)$ \\
$\mathrm{Cl}(4)$ & $\mathrm{C}(702)$ & $1.79(3)$ & $\mathrm{P}(11)$ & $\mathrm{F}(12)$ & $1.597(9)$ \\
$\mathrm{Cl}(5)$ & $\mathrm{C}(703)$ & $1.66(3)$ & $\mathrm{P}(11)$ & $\mathrm{F}(13)$ & $1.573(9)$ \\
$\mathrm{Cl}(6)$ & $\mathrm{C}(703)$ & $1.87(3)$ & $\mathrm{P}(11)$ & $\mathrm{F}(14)$ & $1.590(9)$ \\
$\mathrm{Cl}(7)$ & $\mathrm{C}(704)$ & $1.63(4)$ & $\mathrm{P}(11)$ & $\mathrm{F}(15)$ & $1.609(9)$ \\
$\mathrm{Cl}(8)$ & $\mathrm{C}(704)$ & $1.77(4)$ & $\mathrm{P}(11)$ & $\mathrm{F}(16)$ & $1.592(8)$ \\
$\mathrm{Cl}(9)$ & $\mathrm{C}(705)$ & $1.67(4)$ & $\mathrm{P}(12)$ & $\mathrm{F}(21)$ & $1.564(9)$
\end{tabular}




\begin{tabular}{|c|c|c|c|c|c|}
\hline $\mathrm{Cl}(10)$ & $C(705)$ & $1.50(4)$ & $\mathrm{P}(12)$ & $\mathrm{F}(22)$ & $1.564(9)$ \\
\hline $\mathrm{P}(1)$ & $C(3)$ & $1.83(1)$ & $\mathrm{P}(12)$ & $F(23)$ & $1.606(8)$ \\
\hline $\mathrm{P}(1)$ & $C(111)$ & $1.82(1)$ & $P(12)$ & $\mathrm{F}(24)$ & $1.582(9)$ \\
\hline $\mathrm{P}(1)$ & $C(121)$ & $1.80(1)$ & $\mathrm{P}(12)$ & $F(25)$ & $1.587(8)$ \\
\hline $\mathrm{P}(2)$ & $C(3)$ & $1.83(1)$ & $\mathrm{P}(12)$ & $F(26)$ & $1.569(8)$ \\
\hline $\mathrm{P}(2)$ & $C(4)$ & $1.81(1)$ & $\mathrm{P}(13)$ & $\mathrm{F}(31)$ & $1.52(1)$ \\
\hline$P(2)$ & $C(211)$ & $1.84(1)$ & $P(13)$ & $\mathrm{F}(32)$ & $1.60(2)$ \\
\hline$P(13)$ & $\mathrm{F}(33)$ & $1.53(2)$ & $C(112)$ & $C(113)$ & $1.42(2)$ \\
\hline$P(13)$ & $\mathrm{F}(34)$ & $1.57(1)$ & $C(113)$ & $C(114)$ & $1.38(2)$ \\
\hline$P(13)$ & $\mathrm{F}(35)$ & $1.52(2)$ & $C(114)$ & $C(115)$ & $1.38(2)$ \\
\hline$P(13)$ & $\mathrm{F}(36)$ & $1.56(2)$ & $C(115)$ & $C(116)$ & $1.39(2)$ \\
\hline $\mathrm{N}(1)$ & $C(1)$ & $1.13(1)$ & $C(121)$ & $C(122)$ & $1.41(2)$ \\
\hline $\mathrm{N}(1)$ & $C(11)$ & $1.42(2)$ & $C(121)$ & $C(126)$ & $1.39(2)$ \\
\hline $\mathrm{N}(2)$ & $C(2)$ & $1.12(2)$ & $C(122)$ & $C(123)$ & $1.37(2)$ \\
\hline $\mathrm{N}(2)$ & $C(21)$ & $1.52(2)$ & $C(123)$ & $C(124)$ & $1.41(2)$ \\
\hline$C(11)$ & $C(12)$ & $1.41(2)$ & $C(124)$ & $C(125)$ & $1.38(2)$ \\
\hline$C(11)$ & $C(16)$ & $1.38(2)$ & $C(125)$ & $C(126)$ & $1.39(2)$ \\
\hline$C(12)$ & $C(13)$ & $1.34(2)$ & $C(211)$ & $C(212)$ & $1.42(2)$ \\
\hline$C(12)$ & $C(17)$ & $1.52(3)$ & $C(211)$ & $C(216)$ & $1.36(2)$ \\
\hline$C(13)$ & $C(14)$ & $1.37(3)$ & $C(212)$ & $C(213)$ & $1.42(2)$ \\
\hline$C(14)$ & $C(15)$ & $1.31(3)$ & $C(213)$ & $C(214)$ & $1.39(2)$ \\
\hline$C(15)$ & $C(16)$ & $1.39(2)$ & $C(214)$ & $C(215)$ & $1.41(2)$ \\
\hline$C(16)$ & $C(18)$ & $1.47(2)$ & $C(215)$ & $C(216)$ & $1.41(2)$ \\
\hline$C(21)$ & $C(22)$ & $1.38(2)$ & $C(311)$ & $C(312)$ & $1.36(2)$ \\
\hline$C(21)$ & $C(26)$ & $1.36(2)$ & $C(311)$ & $C(316)$ & $1.39(2)$ \\
\hline$C(22)$ & $C(23)$ & $1.43(3)$ & $C(312)$ & $C(313)$ & $1.40(2)$ \\
\hline$C(22)$ & $C(27)$ & $1.47(3)$ & $C(313)$ & $C(314)$ & $1.37(2)$ \\
\hline$C(23)$ & $C(24)$ & $1.39(3)$ & $C(314)$ & $C(315)$ & $1.35(2)$ \\
\hline$C(24)$ & $C(25)$ & $1.38(3)$ & $C(315)$ & $C(316)$ & $1.42(2)$ \\
\hline$C(25)$ & $C(26)$ & $1.50(3)$ & $C(321)$ & $C(322)$ & $1.42(2)$ \\
\hline$C(26)$ & $C(28)$ & $1.45(3)$ & $C(321)$ & $C(326)$ & $1.39(2)$ \\
\hline$C(111)$ & $C(112)$ & $1.36(2)$ & $C(322)$ & $C(323)$ & $1.32(2)$ \\
\hline$C(111)$ & $C(116)$ & $1.42(2)$ & $C(323)$ & $C(324)$ & $1.38(2)$ \\
\hline$C(324)$ & $C(325)$ & $1.36(2)$ & $C(621)$ & $C(622)$ & $1.38(2)$ \\
\hline$C(325)$ & $C(326)$ & $1.37(2)$ & $C(621)$ & $C(626)$ & $1.44(2)$ \\
\hline$C(411)$ & $C(412)$ & $1.36(2)$ & $C(622)$ & $C(623)$ & $1.38(2)$ \\
\hline$C(411)$ & $C(416)$ & $1.40(2)$ & $C(623)$ & $C(624)$ & $1.40(2)$ \\
\hline$C(412)$ & $C(413)$ & $1.38(2)$ & $C(624)$ & $C(625)$ & $1.39(2)$ \\
\hline$C(413)$ & $C(414)$ & $1.41(2)$ & $C(625)$ & $C(626)$ & $1.39(2)$ \\
\hline$C(414)$ & $C(415)$ & $1.34(2)$ & & & \\
\hline$C(415)$ & $C(416)$ & $1.38(2)$ & & & \\
\hline$C(421)$ & $C(422)$ & $1.40(2)$ & & & \\
\hline$C(421)$ & $C(426)$ & $1.39(2)$ & & & \\
\hline$C(422)$ & $C(423)$ & $1.41(2)$ & & & \\
\hline$C(423)$ & $C(424)$ & $1.40(2)$ & & & \\
\hline$C(424)$ & $C(425)$ & $1.38(2)$ & & & \\
\hline$C(425)$ & $C(426)$ & $1.38(2)$ & & & \\
\hline$C(511)$ & $C(512)$ & $1.41(2)$ & & & \\
\hline$C(511)$ & $C(516)$ & $1.41(2)$ & & & \\
\hline$C(512)$ & $C(513)$ & $1.40(2)$ & & & \\
\hline$C(513)$ & $C(514)$ & $1.39(2)$ & & & \\
\hline$C(514)$ & $C(515)$ & $1.38(2)$ & & & \\
\hline$C(515)$ & $C(516)$ & $1.37(2)$ & & & \\
\hline$C(611)$ & $C(612)$ & $1.43(2)$ & & & \\
\hline$C(611)$ & $C(616)$ & $1.40(2)$ & & & \\
\hline$C(612)$ & $C(613)$ & $1.42(2)$ & & & \\
\hline
\end{tabular}


\begin{tabular}{lll}
$\mathrm{C}(613)$ & $\mathrm{C}(614)$ & $1.29(2)$ \\
$\mathrm{C}(614)$ & $\mathrm{C}(615)$ & $1.33(2)$ \\
$\mathrm{C}(615)$ & $\mathrm{C}(616)$ & $1.44(2)$ \\
\hline
\end{tabular}

a Estimated standard deviations are given in parentheses.

Table S7-5. Bond Angles for Non-hydrogen Atoms of $9 \mathbf{b} \cdot 5 \mathrm{CH}_{2} \mathrm{Cl}_{2}{ }^{\mathrm{a}}$

\begin{tabular}{|c|c|c|c|c|c|c|c|}
\hline atom & atom & atom & angle & atom & atom & atom & angle \\
\hline$\overline{\operatorname{Pt}(2)}$ & $\operatorname{Pt}(1)$ & $\mathrm{P}(1)$ & $89.33(7)$ & $\operatorname{Pt}(2)$ & $\mathrm{P}(2)$ & $\mathrm{C}(211)$ & $113.9(4)$ \\
\hline $\operatorname{Pt}(2)$ & $\operatorname{Pt}(1)$ & $\mathrm{P}(4)$ & $89.22(7)$ & $C(3)$ & $\mathrm{P}(2)$ & $C(4)$ & $104.4(5)$ \\
\hline $\operatorname{Pt}(2)$ & $\operatorname{Pt}(1)$ & $C(1)$ & $160.3(3)$ & $C(3)$ & $\mathrm{P}(2)$ & $C(211)$ & $105.6(5)$ \\
\hline$P(1)$ & $\operatorname{Pt}(1)$ & $\mathrm{P}(4)$ & $176.56(9)$ & $C(4)$ & $P(2)$ & $C(211)$ & $107.3(5)$ \\
\hline$P(1)$ & $\operatorname{Pt}(1)$ & $C(1)$ & $91.4(3)$ & $\operatorname{Pt}(3)$ & $\mathrm{P}(3)$ & $C(4)$ & $114.0(4)$ \\
\hline $\mathrm{P}(4)$ & $\operatorname{Pt}(1)$ & $C(1)$ & $91.1(3)$ & $\operatorname{Pt}(3)$ & $P(3)$ & $C(311)$ & $110.0(4)$ \\
\hline $\operatorname{Pt}(1)$ & $\operatorname{Pt}(2)$ & $\operatorname{Pt}(3)$ & $171.49(2)$ & $\operatorname{Pt}(3)$ & $\mathrm{P}(3)$ & $C(321)$ & $114.2(4)$ \\
\hline $\operatorname{Pt}(1)$ & $\operatorname{Pt}(2)$ & $P(2)$ & $93.61(7)$ & $C(4)$ & $\mathrm{P}(3)$ & $C(311)$ & $104.8(5)$ \\
\hline $\operatorname{Pt}(1)$ & $\operatorname{Pt}(2)$ & $P(5)$ & $92.04(7)$ & $C(4)$ & $\mathrm{P}(3)$ & $C(321)$ & 108.1(5) \\
\hline $\operatorname{Pt}(3)$ & $\operatorname{Pt}(2)$ & $P(2)$ & $86.25(8)$ & $C(311)$ & $\mathrm{P}(3)$ & $C(321)$ & $104.8(5)$ \\
\hline $\operatorname{Pt}(3)$ & $\operatorname{Pt}(2)$ & $P(5)$ & $87.22(8)$ & $\operatorname{Pt}(1)$ & $\mathrm{P}(4)$ & $C(5)$ & $109.6(4)$ \\
\hline$P(2)$ & $\operatorname{Pt}(2)$ & $P(5)$ & 171.5(1) & $\operatorname{Pt}(1)$ & $\mathrm{P}(4)$ & $C(411)$ & $114.4(4)$ \\
\hline $\operatorname{Pt}(2)$ & $\operatorname{Pt}(3)$ & $P(3)$ & $88.62(8)$ & $\operatorname{Pt}(1)$ & $P(4)$ & $C(421)$ & $116.3(3)$ \\
\hline $\operatorname{Pt}(2)$ & $\operatorname{Pt}(3)$ & $P(6)$ & $90.75(8)$ & $C(5)$ & $P(4)$ & $C(411)$ & $105.6(5)$ \\
\hline $\operatorname{Pt}(2)$ & $\operatorname{Pt}(3)$ & $C(2)$ & $178.6(5)$ & $C(5)$ & $\mathrm{P}(4)$ & $C(421)$ & $105.3(5)$ \\
\hline$P(3)$ & $\operatorname{Pt}(3)$ & $P(6)$ & 177.6(1) & $C(411)$ & $\mathrm{P}(4)$ & $C(421)$ & $104.7(5)$ \\
\hline $\mathrm{P}(3)$ & $\operatorname{Pt}(3)$ & $C(2)$ & $92.4(5)$ & $\operatorname{Pt}(2)$ & $P(5)$ & $C(5)$ & $115.7(4)$ \\
\hline$P(6)$ & $\operatorname{Pt}(3)$ & $C(2)$ & $88.2(5)$ & $\operatorname{Pt}(2)$ & $P(5)$ & $C(6)$ & $110.1(4)$ \\
\hline $\operatorname{Pt}(1)$ & $\mathrm{P}(1)$ & $C(3)$ & $112.8(4)$ & $\operatorname{Pt}(2)$ & $P(5)$ & $C(511)$ & $117.5(4)$ \\
\hline $\operatorname{Pt}(1)$ & $\mathrm{P}(1)$ & $C(111)$ & $110.4(4)$ & $C(5)$ & $P(5)$ & $C(6)$ & $100.8(5)$ \\
\hline $\operatorname{Pt}(1)$ & $\mathrm{P}(1)$ & $C(121)$ & $116.4(4)$ & $C(5)$ & $P(5)$ & $C(511)$ & $102.7(5)$ \\
\hline$C(3)$ & $\mathrm{P}(1)$ & $C(111)$ & $106.2(5)$ & $C(6)$ & $P(5)$ & $C(511)$ & $108.6(5)$ \\
\hline$C(3)$ & $P(1)$ & $C(121)$ & $107.4(5)$ & $\operatorname{Pt}(3)$ & $P(6)$ & $C(6)$ & $113.1(4)$ \\
\hline$C(111)$ & $P(1)$ & $C(121)$ & $102.7(5)$ & $\operatorname{Pt}(3)$ & $P(6)$ & $C(611)$ & $113.1(4)$ \\
\hline $\operatorname{Pt}(2)$ & $P(2)$ & $C(3)$ & $114.1(4)$ & $\operatorname{Pt}(3)$ & $P(6)$ & $C(621)$ & $111.4(4)$ \\
\hline $\operatorname{Pt}(2)$ & $P(2)$ & $C(4)$ & $110.8(4)$ & $C(6)$ & $P(6)$ & $C(611)$ & $109.8(6)$ \\
\hline$C(6)$ & $P(6)$ & $C(621)$ & $104.4(6)$ & $\mathrm{F}(23)$ & $P(12)$ & $\mathrm{F}(24)$ & $89.6(5)$ \\
\hline$C(611)$ & $P(6)$ & $C(621)$ & $104.3(6)$ & $\mathrm{F}(23)$ & $\mathrm{P}(12)$ & $F(25)$ & $90.4(5)$ \\
\hline $\mathrm{F}(11)$ & $\mathrm{P}(11)$ & $F(12)$ & $88.4(6)$ & $\mathrm{F}(23)$ & $\mathrm{P}(12)$ & $F(26)$ & $179.4(5)$ \\
\hline $\mathrm{F}(11)$ & $\mathrm{P}(11)$ & $F(13)$ & $177.8(6)$ & $\mathrm{F}(24)$ & $\mathrm{P}(12)$ & $F(25)$ & $88.2(5)$ \\
\hline $\mathrm{F}(11)$ & $\mathrm{P}(11)$ & $\mathrm{F}(14)$ & $90.2(6)$ & $\mathrm{F}(24)$ & $P(12)$ & $\mathrm{F}(26)$ & $90.3(6)$ \\
\hline $\mathrm{F}(11)$ & $\mathrm{P}(11)$ & $\mathrm{F}(15)$ & $88.8(5)$ & $\mathrm{F}(25)$ & $\mathrm{P}(12)$ & $\mathrm{F}(26)$ & $89.0(5)$ \\
\hline $\mathrm{F}(11)$ & $\mathrm{P}(11)$ & $\mathrm{F}(16)$ & $90.6(5)$ & $\mathrm{F}(31)$ & $P(13)$ & $\mathrm{F}(32)$ & $90.1(9)$ \\
\hline $\mathrm{F}(12)$ & $\mathrm{P}(11)$ & $\mathrm{F}(13)$ & $90.6(5)$ & $\mathrm{F}(31)$ & $P(13)$ & $\mathrm{F}(33)$ & $93(1)$ \\
\hline $\mathrm{F}(12)$ & $\mathrm{P}(11)$ & $\mathrm{F}(14)$ & $177.6(5)$ & $\mathrm{F}(31)$ & $P(13)$ & $\mathrm{F}(34)$ & 177(1) \\
\hline $\mathrm{F}(12)$ & $\mathrm{P}(11)$ & $\mathrm{F}(15)$ & $88.3(5)$ & $\mathrm{F}(31)$ & $P(13)$ & $\mathrm{F}(35)$ & $89(1)$ \\
\hline $\mathrm{F}(12)$ & $P(11)$ & $\mathrm{F}(16)$ & $91.5(4)$ & $\mathrm{F}(31)$ & $P(13)$ & $\mathrm{F}(36)$ & $93(1)$ \\
\hline$F(13)$ & $\mathrm{P}(11)$ & $\mathrm{F}(14)$ & $90.7(5)$ & $\mathrm{F}(32)$ & $\mathrm{P}(13)$ & $\mathrm{F}(33)$ & $92(1)$ \\
\hline $\mathrm{F}(13)$ & $P(11)$ & $\mathrm{F}(15)$ & $89.1(5)$ & $\mathrm{F}(32)$ & $P(13)$ & $\mathrm{F}(34)$ & $91(1)$ \\
\hline $\mathrm{F}(13)$ & $\mathrm{P}(11)$ & $\mathrm{F}(16)$ & $91.4(5)$ & $\mathrm{F}(32)$ & $P(13)$ & $\mathrm{F}(35)$ & $176(1)$ \\
\hline $\mathrm{F}(14)$ & $\mathrm{P}(11)$ & $F(15)$ & $89.6(5)$ & $\mathrm{F}(32)$ & $P(13)$ & $\mathrm{F}(36)$ & $86(1)$ \\
\hline $\mathrm{F}(14)$ & $\mathrm{P}(11)$ & $\mathrm{F}(16)$ & $90.5(5)$ & $\mathrm{F}(33)$ & $P(13)$ & $\mathrm{F}(34)$ & $89(1)$ \\
\hline $\mathrm{F}(15)$ & $\mathrm{P}(11)$ & $F(16)$ & $179.4(5)$ & $\mathrm{F}(33)$ & $P(13)$ & $\mathrm{F}(35)$ & 91(1) \\
\hline $\mathrm{F}(21)$ & $\mathrm{P}(12)$ & $\mathrm{F}(22)$ & $88.9(5)$ & $\mathrm{F}(33)$ & $P(13)$ & $\mathrm{F}(36)$ & 174(1) \\
\hline $\mathrm{F}(21)$ & $\mathrm{P}(12)$ & $\mathrm{F}(23)$ & $88.5(5)$ & $\mathrm{F}(34)$ & $P(13)$ & $\mathrm{F}(35)$ & $90(1)$ \\
\hline $\mathrm{F}(21)$ & $\mathrm{P}(12)$ & $\mathrm{F}(24)$ & $178.1(6)$ & $\mathrm{F}(34)$ & $P(13)$ & $F(36)$ & $85(1)$ \\
\hline $\mathrm{F}(21)$ & $\mathrm{P}(12)$ & $F(25)$ & $91.4(5)$ & $\mathrm{F}(35)$ & $\mathrm{P}(13)$ & $\mathrm{F}(36)$ & $90(1)$ \\
\hline $\mathrm{F}(21)$ & $P(12)$ & $\mathrm{F}(26)$ & $91.5(6)$ & $C(1)$ & $\mathrm{N}(1)$ & $C(11)$ & $166(1)$ \\
\hline
\end{tabular}




\begin{tabular}{|c|c|c|c|c|c|c|c|}
\hline $\mathrm{F}(22)$ & $\mathrm{P}(12)$ & $\mathrm{F}(23)$ & $89.5(5)$ & $C(2)$ & $N(2)$ & $C(21)$ & $178(1)$ \\
\hline $\mathrm{F}(22)$ & $\mathrm{P}(12)$ & $\mathrm{F}(24)$ & 91.5(5) & $\operatorname{Pt}(1)$ & $C(1)$ & $\mathrm{N}(1)$ & $174(1)$ \\
\hline $\mathrm{F}(22)$ & $\mathrm{P}(12)$ & $\mathrm{F}(25)$ & 179.6(6) & $\operatorname{Pt}(3)$ & $C(2)$ & $\mathrm{N}(2)$ & 171(1) \\
\hline $\mathrm{F}(22)$ & $\mathrm{P}(12)$ & $\mathrm{F}(26)$ & 91.1(5) & $\mathrm{P}(1)$ & $C(3)$ & $\mathrm{P}(2)$ & $110.7(6)$ \\
\hline $\mathrm{P}(2)$ & $C(4)$ & $\mathrm{P}(3)$ & 107.7(6) & $C(25)$ & $C(26)$ & $C(28)$ & $119(2)$ \\
\hline $\mathrm{P}(4)$ & $C(5)$ & $P(5)$ & $109.8(6)$ & $\mathrm{P}(1)$ & $C(111)$ & $C(112)$ & $120.9(9)$ \\
\hline $\mathrm{P}(5)$ & $C(6)$ & $P(6)$ & 110.6(6) & $\mathrm{P}(1)$ & $C(111)$ & $C(116)$ & $118.4(8)$ \\
\hline $\mathrm{N}(1)$ & $C(11)$ & $C(12)$ & 117(1) & $C(112)$ & $C(111)$ & $C(116)$ & $120(1)$ \\
\hline $\mathrm{N}(1)$ & $C(11)$ & $C(16)$ & $119(1)$ & $C(111)$ & $C(112)$ & $C(113)$ & $120(1)$ \\
\hline$C(12)$ & $C(11)$ & $C(16)$ & $124(1)$ & $C(112)$ & $C(113)$ & $C(114)$ & 119(1) \\
\hline$C(11)$ & $C(12)$ & $C(13)$ & 115(2) & $C(113)$ & $C(114)$ & $C(115)$ & $122(1)$ \\
\hline$C(11)$ & $C(12)$ & $C(17)$ & $122(2)$ & $C(114)$ & $C(115)$ & $C(116)$ & $119(1)$ \\
\hline$C(13)$ & $C(12)$ & $C(17)$ & $124(2)$ & $C(111)$ & $C(116)$ & $C(115)$ & $120(1)$ \\
\hline$C(12)$ & $C(13)$ & $C(14)$ & 122(2) & $\mathrm{P}(1)$ & $C(121)$ & $C(122)$ & 119.2(9) \\
\hline$C(13)$ & $C(14)$ & $C(15)$ & $122(2)$ & $\mathrm{P}(1)$ & $C(121)$ & $C(126)$ & 121.1(9) \\
\hline$C(14)$ & $C(15)$ & $C(16)$ & $121(2)$ & $C(122)$ & $C(121)$ & $C(126)$ & $120(1)$ \\
\hline$C(11)$ & $C(16)$ & $C(15)$ & $116(2)$ & $C(121)$ & $C(122)$ & $C(123)$ & 120(1) \\
\hline$C(11)$ & $C(16)$ & $C(18)$ & 121(1) & $C(122)$ & $C(123)$ & $C(124)$ & 120(1) \\
\hline$C(15)$ & $C(16)$ & $C(18)$ & $123(2)$ & $C(123)$ & $C(124)$ & $C(125)$ & 121(1) \\
\hline $\mathrm{N}(2)$ & $C(21)$ & $C(22)$ & 118(2) & $C(124)$ & $C(125)$ & $C(126)$ & 119(1) \\
\hline $\mathrm{N}(2)$ & $C(21)$ & $C(26)$ & $116(2)$ & $C(121)$ & $C(126)$ & $C(125)$ & 121(1) \\
\hline$C(22)$ & $C(21)$ & $C(26)$ & $126(2)$ & $\mathrm{P}(2)$ & $C(211)$ & $C(212)$ & 116(1) \\
\hline$C(21)$ & $C(22)$ & $C(23)$ & $116(2)$ & $\mathrm{P}(2)$ & $C(211)$ & $C(216)$ & 123(1) \\
\hline$C(21)$ & $C(22)$ & $C(27)$ & 121(2) & $C(212)$ & $C(211)$ & $C(216)$ & 121(1) \\
\hline$C(23)$ & $C(22)$ & $C(27)$ & $123(2)$ & $C(211)$ & $C(212)$ & $C(213)$ & $118(1)$ \\
\hline$C(22)$ & $C(23)$ & $C(24)$ & 120(2) & $C(212)$ & $C(213)$ & $C(214)$ & 118(2) \\
\hline$C(23)$ & $C(24)$ & $C(25)$ & $125(2)$ & $C(213)$ & $C(214)$ & $C(215)$ & $124(2)$ \\
\hline$C(24)$ & $C(25)$ & $C(26)$ & 115(2) & $C(214)$ & $C(215)$ & $C(216)$ & 115(2) \\
\hline$C(21)$ & $C(26)$ & $C(25)$ & $118(2)$ & $C(211)$ & $C(216)$ & $C(215)$ & $122(1)$ \\
\hline$C(21)$ & $C(26)$ & $C(28)$ & 123(2) & $\mathrm{P}(3)$ & $C(311)$ & $C(312)$ & 118.1(9) \\
\hline $\mathrm{P}(3)$ & $C(311)$ & $C(316)$ & $120.6(9)$ & $C(421)$ & $C(422)$ & $C(423)$ & 119(1) \\
\hline$C(312)$ & $C(311)$ & $C(316)$ & 121(1) & $C(422)$ & $C(423)$ & $C(424)$ & 121(1) \\
\hline$C(311)$ & $C(312)$ & $C(313)$ & $122(1)$ & $C(423)$ & $C(424)$ & $C(425)$ & $118(1)$ \\
\hline$C(312)$ & $C(313)$ & $C(314)$ & 117(1) & $C(424)$ & $C(425)$ & $C(426)$ & $122(1)$ \\
\hline$C(313)$ & $C(314)$ & $C(315)$ & 122(2) & $C(421)$ & $C(426)$ & $C(425)$ & 121(1) \\
\hline$C(314)$ & $C(315)$ & $C(316)$ & 121(1) & $\mathrm{P}(5)$ & $C(511)$ & $C(512)$ & $118.9(9)$ \\
\hline$C(311)$ & $C(316)$ & $C(315)$ & 117(1) & $P(5)$ & $C(511)$ & $C(516)$ & $121.4(9)$ \\
\hline $\mathrm{P}(3)$ & $C(321)$ & $C(322)$ & 122(1) & $C(512)$ & $C(511)$ & $C(516)$ & 119(1) \\
\hline $\mathrm{P}(3)$ & $C(321)$ & $C(326)$ & 120(1) & $C(511)$ & $C(512)$ & $C(513)$ & $120(1)$ \\
\hline$C(322)$ & $C(321)$ & $C(326)$ & 118(1) & $C(512)$ & $C(513)$ & $C(514)$ & 119(1) \\
\hline$C(321)$ & $C(322)$ & $C(323)$ & 120(1) & $C(513)$ & $C(514)$ & $C(515)$ & $122(1)$ \\
\hline$C(322)$ & $C(323)$ & $C(324)$ & $121(2)$ & $C(514)$ & $C(515)$ & $C(516)$ & $120(1)$ \\
\hline$C(323)$ & C(324) & $C(325)$ & $121(2)$ & $C(511)$ & $C(516)$ & $C(515)$ & $120(1)$ \\
\hline$C(324)$ & $C(325)$ & $C(326)$ & 119(2) & $P(6)$ & $C(611)$ & $C(612)$ & $120(1)$ \\
\hline$C(321)$ & $C(326)$ & $C(325)$ & 121(1) & $P(6)$ & $C(611)$ & $C(616)$ & $122(1)$ \\
\hline $\mathrm{P}(4)$ & $C(411)$ & $C(412)$ & 121.4(9) & $C(612)$ & $C(611)$ & $C(616)$ & $118(1)$ \\
\hline $\mathrm{P}(4)$ & $C(411)$ & $C(416)$ & 120.0(9) & $C(611)$ & $C(612)$ & $C(613)$ & 119(1) \\
\hline$C(412)$ & $C(411)$ & $C(416)$ & 118(1) & $C(612)$ & $C(613)$ & $C(614)$ & $120(2)$ \\
\hline$C(411)$ & $C(412)$ & $C(413)$ & 123(1) & $C(613)$ & $C(614)$ & $C(615)$ & $125(2)$ \\
\hline$C(412)$ & $C(413)$ & $C(414)$ & 117(1) & $C(614)$ & $C(615)$ & $C(616)$ & $118(2)$ \\
\hline$C(413)$ & $C(414)$ & $C(415)$ & 121(1) & $C(611)$ & $C(616)$ & $C(615)$ & 119(1) \\
\hline$C(414)$ & $C(415)$ & $C(416)$ & 121(1) & $\mathrm{P}(6)$ & $C(621)$ & $C(622)$ & 122(1) \\
\hline$C(411)$ & $C(416)$ & $C(415)$ & 120(1) & $P(6)$ & $C(621)$ & $C(626)$ & 119(1) \\
\hline $\mathrm{P}(4)$ & $C(421)$ & $C(422)$ & $120.0(8)$ & $C(622)$ & $C(621)$ & $C(626)$ & $119(1)$ \\
\hline $\mathrm{P}(4)$ & $C(421)$ & $C(426)$ & $120.8(8)$ & $C(621)$ & $C(622)$ & $C(623)$ & 121(1) \\
\hline$C(422)$ & $C(421)$ & $C(426)$ & 119(1) & $C(622)$ & $C(623)$ & $C(624)$ & $120(2)$ \\
\hline
\end{tabular}


$\mathrm{C}(623) \quad \mathrm{C}(624) \quad \mathrm{C}(625) \quad 122(2)$

C(624) C(625) C(626) 118(2)

C(621) C(626) C(625) 121(1)

$\mathrm{Cl}(1) \quad \mathrm{C}(701) \quad \mathrm{Cl}(2) \quad 111(1)$

$\mathrm{Cl}(3) \quad \mathrm{C}(702) \quad \mathrm{Cl}(4) \quad 109(1)$

$\mathrm{Cl}(5) \quad \mathrm{C}(703) \quad \mathrm{Cl}(6) \quad 107(2)$

$\mathrm{Cl}(7) \quad \mathrm{C}(704) \quad \mathrm{Cl}(8) \quad 114(2)$

$\mathrm{Cl}(9) \quad \mathrm{C}(705) \quad \mathrm{Cl}(10) \quad 117(3)$

a Estimated deviations are given in parentheses. 
Table S8-1. Crystallographic and Experimental Data for $\left[\mathrm{Pt}_{3}(\mu-\mathrm{dpmp})_{2}(\mu-\mathrm{NO})_{2}(\mathrm{XylNC})_{2}\right]-$ $\left(\mathrm{BF}_{4}\right)_{4} \cdot \mathrm{CH}_{2} \mathrm{Cl}_{2} \cdot 3 \mathrm{H}_{2} \mathrm{O}\left(\mathbf{1 0 a} \cdot \mathrm{CH}_{2} \mathrm{Cl}_{2} \cdot 3 \mathrm{H}_{2} \mathrm{O}\right)$

\begin{tabular}{|c|c|}
\hline formula & $\mathrm{C}_{83} \mathrm{H}_{84} \mathrm{~N}_{4} \mathrm{O}_{5} \mathrm{P}_{6} \mathrm{~B}_{4} \mathrm{~F}_{16} \mathrm{Cl}_{2} \mathrm{Pt}_{3}$ \\
\hline formula weight & 2406.83 \\
\hline color & pale green \\
\hline size, $\mathrm{mm}$ & $0.42 \times 0.38 \times 0.30$ \\
\hline cryst. system & monoclinic \\
\hline space group & $P 21 / c$ (No. 14$)$ \\
\hline no. refs used for cell determination & $25\left(20<2 \theta<30^{\circ}\right)$ \\
\hline lattice const. & $\begin{array}{l}a=12.873(4) \AA \\
b=29.350(6) \AA \\
c=14.826(4) \AA \\
\beta=110.49(3){ }^{\circ} \\
V=5246(2) \AA^{3}\end{array}$ \\
\hline Z & 2 \\
\hline$D_{\text {calc }}, \mathrm{g} \mathrm{cm}^{-3}$ & 1.523 \\
\hline$\mu(\mathrm{Mo} \mathrm{K \alpha}), \mathrm{cm}^{-1}$ & 41.89 (trans. $0.67 \sim 0.99$ ) \\
\hline \multicolumn{2}{|c|}{ An absorption correction by the $\psi$-scan method was applied. } \\
\hline diffractometer & Rigaku AFC7R (50 kV 200 mA) \\
\hline radiation & $\operatorname{Mo} K \alpha(1=0.71069 \AA)$ \\
\hline monochromator & graphite \\
\hline temp, ${ }^{\circ} \mathrm{C}$ & -116 \\
\hline $2 \theta$ range $^{\circ}$ & $4<2 \theta<50$ \\
\hline $\begin{array}{l}h, k, l \text { range } \\
\text { scan method }\end{array}$ & $\begin{array}{l}+h,+k, \pm l \\
\omega-2 \theta\end{array}$ \\
\hline scan speed, ${ }^{\circ} \min ^{-1}$ & 8 \\
\hline scan width & $1.10+0.30 \tan \theta$ \\
\hline std. reflections & 3 every 150 refs \\
\hline$F_{000}$ & 2344.00 \\
\hline$R_{\text {int }}$ & 0.031 \\
\hline solution & Direct Methods (SIR92) \\
\hline \multirow[t]{2}{*}{ refinement } & full-matrix least-squares \\
\hline & $\begin{array}{l}\text { All non-hydrogen atoms except } \mathrm{BF}_{4} \text { anions and } \mathrm{H}_{2} \mathrm{O} \\
\text { molecules were refined with aniotropic } \\
\text { temperature factors. }\end{array}$ \\
\hline hydrogen atoms & $\begin{array}{l}\text { All C-H hydrogen atoms were calculated at ideal } \\
\text { positions with } \mathrm{C}-\mathrm{H}=0.95 \AA \text {, and were not refined. }\end{array}$ \\
\hline function minimized & $\Sigma w\left(\left|F_{o}\right|-\left|F_{c}\right|\right)^{2}$ \\
\hline weighting scheme & $1 / \sigma^{2}\left(F_{o}\right)$ \\
\hline anomalous dispersion & all non-hydrogen atoms \\
\hline no. unique reflections & 9211 \\
\hline no. obsd. unique reflections & $6842(I>3 \sigma(I))$ \\
\hline$p$-factor & 0.03 \\
\hline no. variables & 492 \\
\hline data / param ratio & 13.91 \\
\hline$R^{\mathrm{a}}$ & 0.058 \\
\hline$R_{\mathrm{W}} \mathrm{b}$ & 0.072 \\
\hline GOF $\mathrm{c}$ & 2.53 \\
\hline$\rho_{\max }, \mathrm{e}^{-3}$ & $1.14,-1.30$ \\
\hline
\end{tabular}

a $R=\Sigma|| F_{\mathrm{O}}|-| F_{\mathrm{C}}|| / \Sigma\left|F_{\mathrm{O}}\right|$.

$\mathrm{b}_{R_{\mathrm{W}}}=\left[\Sigma w\left(\left|F_{\mathrm{O}}\right|-\left|F_{\mathrm{C}}\right|\right)^{2} / \Sigma w\left|F_{\mathrm{O}}\right|^{2}\right]^{1 / 2}\left(w=1 / \sigma^{2}\left(F_{\mathrm{O}}\right)\right)$.

${ }^{c} \mathrm{GOF}=\left[\Sigma w\left(\left|F_{\mathrm{O}}\right|-\left|F_{\mathrm{C}}\right|\right)^{2} /\left(N_{\mathrm{O}}-N_{\mathrm{p}}\right]^{1 / 2}\left(N_{\mathrm{O}}=\right.\right.$ no. data, $N_{\mathrm{p}}=$ no. variables $)$. 
Table S8-2. Final Positional Parameters and $B$ eq for $\left[\mathrm{Pt}_{3}(\mu \text {-dpmp })_{2}(\mu-\mathrm{NO})_{2}\left(\mathrm{XylNC}_{2}\right]\left(\mathrm{BF}_{4}\right)_{4}\right.$ $\mathrm{CH}_{2} \mathrm{Cl}_{2} \cdot 3 \mathrm{H}_{2} \mathrm{O}\left(\mathbf{1 0 a} \cdot \mathrm{CH}_{2} \mathrm{Cl}_{2} \cdot 3 \mathrm{H}_{2} \mathrm{O}\right){ }^{\mathrm{a}}, \mathrm{b}, \mathrm{c}$

\begin{tabular}{|c|c|c|c|c|}
\hline atom & $x$ & $y$ & $\bar{z}$ & $B$ eq \\
\hline$\overline{\operatorname{Pt}(1)}$ & $0.17170(2)$ & $0.074564(9)$ & $0.10039(2)$ & $2.587(6)$ \\
\hline $\operatorname{Pt}(2)$ & 0.0000 & 0.0000 & 0.0000 & $2.680(9)$ \\
\hline $\mathrm{Cl}(1)^{\mathrm{e}}$ & $0.3904(9)$ & $0.1513(3)$ & $0.4854(10)$ & $15.6(4)$ \\
\hline $\mathrm{Cl}(2)^{\mathrm{e}}$ & $0.495(1)$ & $0.1391(3)$ & $0.6841(9)$ & $16.2(4)$ \\
\hline$P(1)$ & $0.1600(2)$ & $0.10027(6)$ & $-0.0535(1)$ & $2.71(4)$ \\
\hline $\mathrm{P}(2)$ & $-0.0022(2)$ & $0.02091(6)$ & $-0.1517(1)$ & $2.71(4)$ \\
\hline $\mathrm{P}(3)$ & $0.2013(2)$ & $0.04073(6)$ & $0.2505(1)$ & $2.75(4)$ \\
\hline $\mathrm{F}(1)^{\mathrm{d}}$ & $0.9430(5)$ & $0.1063(3)$ & $0.6258(6)$ & $9.02^{d}$ \\
\hline$F(2)^{d}$ & $0.7691(7)$ & $0.1100(3)$ & $0.6218(6)$ & $9.02^{d}$ \\
\hline$F(3)^{d}$ & $0.8080(7)$ & $0.1260(3)$ & $0.4906(5)$ & $9.02^{d}$ \\
\hline $\mathrm{F}(4)^{\mathrm{d}}$ & $0.8198(8)$ & $0.0547(2)$ & $0.5437(6)$ & $9.02^{\mathrm{d}}$ \\
\hline$F(5)^{d}$ & $0.8755(8)$ & $0.1089(5)$ & $0.9656(9)$ & $14.70^{d}$ \\
\hline$F(6)^{d}$ & $0.706(1)$ & $0.1243(5)$ & $0.9649(9)$ & $14.70^{\mathrm{d}}$ \\
\hline $\mathrm{F}(7)^{\mathrm{d}}$ & $0.7341(1)$ & $0.0828(5)$ & $0.8423(9)$ & $14.70^{d}$ \\
\hline$F(8)^{d}$ & $0.7741(1)$ & $0.1567(5)$ & $0.8552(9)$ & $14.70^{d}$ \\
\hline $\mathrm{O}(1)$ & $0.2361(6)$ & $-0.0112(3)$ & $0.0559(6)$ & $7.3(2)$ \\
\hline $\mathrm{O}(11)^{\mathrm{e}}$ & $0.530(1)$ & $0.2178(4)$ & $0.1205(9)$ & $5.3(3)$ \\
\hline $\mathrm{O}(12)^{\mathrm{e}}$ & $0.819(2)$ & $0.1962(7)$ & $0.396(1)$ & $9.7(5)$ \\
\hline $\mathrm{O}(13)^{\mathrm{e}}$ & $0.621(1)$ & $0.1756(6)$ & $-0.031(1)$ & $9.2(4)$ \\
\hline $\mathrm{N}(1)$ & $0.1653(5)$ & $0.0108(2)$ & $0.0474(4)$ & $2.66(8)$ \\
\hline $\mathrm{N}(2)$ & $0.1695(6)$ & $0.1741(2)$ & $0.1619(5)$ & $4.2(1)$ \\
\hline$C(1)$ & $0.1707(7)$ & $0.1365(3)$ & $0.1425(5)$ & $3.4(2)$ \\
\hline$C(2)$ & $0.0358(6)$ & $0.0809(2)$ & $-0.1492(5)$ & $3.1(1)$ \\
\hline$C(3)$ & $0.1400(6)$ & $-0.0166(2)$ & $0.2389(5)$ & $3.0(2)$ \\
\hline$C(11)$ & $0.156(1)$ & $0.2217(3)$ & $0.1751(6)$ & $5.6(2)$ \\
\hline$C(12)$ & $0.047(1)$ & $0.2384(4)$ & $0.1489(8)$ & $7.2(3)$ \\
\hline$C(13)$ & $0.030(1)$ & $0.2857(4)$ & $0.1532(9)$ & $8.9(4)$ \\
\hline$C(14)$ & $0.124(2)$ & $0.3113(4)$ & $0.1866(9)$ & $9.7(4)$ \\
\hline$C(15)$ & $0.235(2)$ & $0.2953(4)$ & $0.2158(9)$ & $9.7(4)$ \\
\hline$C(16)$ & $0.248(1)$ & $0.2457(4)$ & $0.2075(8)$ & $7.8(3)$ \\
\hline$C(17)$ & $-0.039(1)$ & $0.2106(5)$ & $0.120(1)$ & $10.2(5)$ \\
\hline$C(18)$ & $0.356(1)$ & $0.2274(5)$ & $0.236(1)$ & $11.3(5)$ \\
\hline$C(111)$ & $0.1591(7)$ & $0.1619(2)$ & $-0.0656(5)$ & $3.2(1)$ \\
\hline$C(112)$ & $0.2622(7)$ & $0.1841(3)$ & $-0.0413(7)$ & $4.6(2)$ \\
\hline$C(113)$ & $0.2623(8)$ & $0.2318(3)$ & $-0.0439(7)$ & $5.5(2)$ \\
\hline$C(114)$ & $0.1684(9)$ & $0.2549(3)$ & $-0.0679(7)$ & $4.8(2)$ \\
\hline$C(115)$ & $0.0686(8)$ & $0.2332(3)$ & $-0.0921(7)$ & $5.3(2)$ \\
\hline$C(116)$ & $0.0610(7)$ & $0.1872(3)$ & $-0.0911(6)$ & $4.5(2)$ \\
\hline$C(121)$ & $0.2778(6)$ & $0.0798(2)$ & $-0.0795(5)$ & $3.1(1)$ \\
\hline$C(122)$ & $0.2766(8)$ & $0.0757(3)$ & $-0.1745(6)$ & $4.4(2)$ \\
\hline$C(123)$ & $0.3705(9)$ & $0.0622(3)$ & $-0.1915(8)$ & $5.5(2)$ \\
\hline$C(124)$ & $0.4643(9)$ & $0.0536(4)$ & $-0.1193(9)$ & $6.0(2)$ \\
\hline$C(125)$ & $0.4699(8)$ & $0.0576(4)$ & $-0.0271(9)$ & $6.2(3)$ \\
\hline$C(126)$ & $0.3748(7)$ & $0.0704(3)$ & $-0.0049(6)$ & $4.3(2)$ \\
\hline$C(211)$ & $0.0841(6)$ & $-0.0110(2)$ & $-0.2039(5)$ & $3.0(1)$ \\
\hline$C(212)$ & $0.0796(7)$ & $-0.0007(3)$ & $-0.2960(6)$ & $3.9(2)$ \\
\hline$C(213)$ & $0.1401(8)$ & $-0.0269(3)$ & $-0.3372(7)$ & $4.8(2)$ \\
\hline$C(214)$ & $0.2088(9)$ & $-0.0602(3)$ & $-0.2873(8)$ & $5.6(2)$ \\
\hline$C(215)$ & $0.2164(8)$ & $-0.0705(3)$ & $-0.1931(8)$ & $5.8(2)$ \\
\hline$C(216)$ & $0.1533(7)$ & $-0.0458(3)$ & $-0.1520(6)$ & $4.2(2)$ \\
\hline$C(311)$ & $0.1540(6)$ & $0.0704(2)$ & $0.3339(5)$ & $3.1(1)$ \\
\hline$C(312)$ & $0.0700(7)$ & $0.1037(3)$ & $0.3036(6)$ & $4.1(2)$ \\
\hline
\end{tabular}




\begin{tabular}{|c|c|c|c|c|}
\hline$C(313)$ & $0.0302(8)$ & $0.1252(3)$ & $0.3668(8)$ & $5.3(2)$ \\
\hline$C(314)$ & $0.074(1)$ & $0.1155(4)$ & $0.4631(9)$ & $6.8(3)$ \\
\hline$C(315)$ & $0.165(1)$ & $0.0836(4)$ & $0.4976(7)$ & 7.1(3) \\
\hline$C(316)$ & $0.2006(8)$ & $0.0611(3)$ & $0.4335(6)$ & $4.8(2)$ \\
\hline$C(321)$ & $0.3492(6)$ & $0.0318(3)$ & $0.3072(5)$ & $3.2(1)$ \\
\hline$C(322)$ & $0.4170(7)$ & $0.0683(3)$ & $0.3139(8)$ & $5.7(2)$ \\
\hline$C(323)$ & $0.5297(7)$ & $0.0651(4)$ & $0.3573(9)$ & $6.8(3)$ \\
\hline$C(324)$ & $0.5784(7)$ & $0.0239(4)$ & $0.3943(7)$ & $5.0(2)$ \\
\hline$C(325)$ & $0.5113(7)$ & $-0.0134(3)$ & $0.3837(7)$ & $4.7(2)$ \\
\hline$C(326)$ & $0.3971(7)$ & $-0.0100(3)$ & $0.3409(6)$ & $3.8(2)$ \\
\hline$C(406)^{f}$ & $0.389(2)$ & $0.1451(10)$ & $0.587(2)$ & $2.8(5)$ \\
\hline$C(407)^{f}$ & $0.514(3)$ & $0.166(1)$ & $0.609(3)$ & $5.1(8)$ \\
\hline$B(1)^{d}$ & $0.8350(5)$ & $0.0993(2)$ & $0.5705(4)$ & $9.02^{\mathrm{d}}$ \\
\hline$B(2)^{d}$ & $0.7719(7)$ & $0.1228(3)$ & $0.9103(6)$ & $14.70^{d}$ \\
\hline $\mathrm{H}(2)$ & 0.1695 & -0.0356 & 0.2030 & 3.16 \\
\hline $\mathrm{H}(3)$ & 0.1587 & -0.0295 & 0.3036 & 3.16 \\
\hline $\mathrm{H}(4)$ & -0.1204 & 0.2224 & 0.0986 & 11.38 \\
\hline $\mathrm{H}(5)$ & -0.0543 & 0.1880 & 0.0602 & 11.38 \\
\hline $\mathrm{H}(6)$ & -0.0504 & 0.1846 & 0.1660 & 11.38 \\
\hline $\mathrm{H}(7)$ & 0.3604 & 0.2033 & 0.2825 & 11.26 \\
\hline $\mathrm{H}(8)$ & 0.3568 & 0.2111 & 0.1777 & 11.26 \\
\hline $\mathrm{H}(9)$ & 0.4061 & 0.2482 & 0.2557 & 11.26 \\
\hline $\mathrm{H}(10)$ & -0.0263 & 0.0985 & -0.1470 & 3.64 \\
\hline $\mathrm{H}(11)$ & 0.0443 & 0.0875 & -0.2104 & 3.64 \\
\hline $\mathrm{H}(12)$ & -0.0323 & 0.3047 & 0.1414 & 10.66 \\
\hline $\mathrm{H}(13)$ & 0.1187 & 0.3422 & 0.1930 & 8.97 \\
\hline $\mathrm{H}(14)$ & 0.2878 & 0.3218 & 0.2342 & 8.83 \\
\hline $\mathrm{H}(15)$ & 0.3254 & 0.1655 & -0.0236 & 4.78 \\
\hline $\mathrm{H}(16)$ & 0.3372 & 0.2461 & -0.0195 & 5.77 \\
\hline $\mathrm{H}(17)$ & 0.1673 & 0.2877 & -0.0750 & 6.69 \\
\hline $\mathrm{H}(18)$ & 0.0005 & 0.2509 & -0.1132 & 6.02 \\
\hline $\mathrm{H}(19)$ & -0.0075 & 0.1694 & -0.1031 & 4.99 \\
\hline $\mathrm{H}(20)$ & 0.2078 & 0.0828 & -0.2278 & 4.55 \\
\hline $\mathrm{H}(21)$ & 0.3715 & 0.0563 & -0.2568 & 5.79 \\
\hline $\mathrm{H}(22)$ & 0.5314 & 0.0469 & -0.1265 & 6.50 \\
\hline $\mathrm{H}(23)$ & 0.5390 & 0.0533 & 0.0263 & 5.89 \\
\hline $\mathrm{H}(24)$ & 0.3773 & 0.0691 & 0.0614 & 4.51 \\
\hline $\mathrm{H}(25)$ & 0.0277 & 0.0224 & -0.3317 & 4.49 \\
\hline $\mathrm{H}(26)$ & 0.1299 & -0.0190 & -0.4062 & 5.20 \\
\hline $\mathrm{H}(27)$ & 0.2545 & -0.0764 & -0.3158 & 6.47 \\
\hline $\mathrm{H}(28)$ & 0.2693 & -0.0933 & -0.1552 & 6.41 \\
\hline $\mathrm{H}(29)$ & 0.1546 & -0.0513 & -0.0846 & 4.05 \\
\hline $\mathrm{H}(30)$ & 0.0364 & 0.1110 & 0.2331 & 3.94 \\
\hline $\mathrm{H}(31)$ & -0.0255 & 0.1505 & 0.3440 & 5.49 \\
\hline $\mathrm{H}(32)$ & 0.0359 & 0.1312 & 0.5016 & 6.72 \\
\hline $\mathrm{H}(33)$ & 0.1905 & 0.0796 & 0.5653 & 8.60 \\
\hline $\mathrm{H}(34)$ & 0.2579 & 0.0363 & 0.4544 & 4.96 \\
\hline $\mathrm{H}(35)$ & 0.3858 & 0.0982 & 0.2902 & 5.64 \\
\hline $\mathrm{H}(36)$ & 0.5738 & 0.0913 & 0.3558 & 6.66 \\
\hline $\mathrm{H}(37)$ & 0.6564 & 0.0233 & 0.4278 & 5.28 \\
\hline $\mathrm{H}(38)$ & 0.5426 & -0.0421 & 0.4086 & 5.72 \\
\hline $\mathrm{H}(39)$ & 0.3478 & -0.0366 & 0.3359 & 4.25 \\
\hline
\end{tabular}

a Estimated standard deviations are given in parentheses. $\mathrm{b}$ All non-hydrogen atoms except $\mathrm{BF}_{4}$ anions and $\mathrm{H}_{2} \mathrm{O}$ molecules were assigned with anisotropic thermal parameters given as the isotropic equivalent displacement parameter defined as $B_{\mathrm{eq}}=\left(8 \pi^{2} / 3\right)\left(U_{11}\left(a a^{*}\right)^{2}+U_{22}\left(b b^{*}\right)^{2}+\right.$ $\left.U_{33}\left(c c^{*}\right)^{2}+2 U_{12} a a^{*} b b^{*} \cos \gamma+2 U_{13} a a^{*} c c^{*} \cos \beta+2 U_{23} b b^{*} c c^{*} \cos \alpha\right)$. c All C-H hydrogen atoms were 
calculated at ideal positions with $\mathrm{C}-\mathrm{H}=0.95 \AA$ and were not refined. $\mathrm{d} \mathrm{BF}_{4}$ anions were treated as rigid groups in refinement. ${ }^{\mathrm{e}}$ Disordered with 0.5 occupancy. ${ }^{\mathrm{f}}$ Disordered with 0.25 occupancy.

Table S8-3. Final Anisotropic Thermal Parameters for [ $\mathrm{Pt}_{3}(\mu \text {-dpmp })_{2}(\mu-$

$\left.\mathrm{NO})_{2}(\mathrm{XylNC})_{2}\right]\left(\mathrm{BF}_{4}\right)_{4} \cdot \mathrm{CH}_{2} \mathrm{Cl}_{2} \cdot 3 \mathrm{H}_{2} \mathrm{O}\left(\mathbf{1 0 a} \cdot \mathrm{CH}_{2} \mathrm{Cl}_{2} \cdot 3 \mathrm{H}_{2} \mathrm{O}\right)^{\mathrm{a}, \mathrm{b}}$

\begin{tabular}{|c|c|c|c|c|c|c|}
\hline atom & $U_{11}$ & $U_{22}$ & $U_{33}$ & $U_{12}$ & $U_{13}$ & $U_{23}$ \\
\hline$\overline{\operatorname{Pt}(1)}$ & $0.0353(2)$ & $0.0299(2)$ & $0.0304(2)$ & $0.0001(1)$ & $0.0081(1)$ & $-0.0003(1)$ \\
\hline $\operatorname{Pt}(2)$ & $0.0392(2)$ & $0.0315(2)$ & $0.0276(2)$ & $-0.0050(2)$ & $0.0072(2)$ & $0.0006(2)$ \\
\hline $\mathrm{Cl}(1)$ & $0.185(9)$ & $0.093(5)$ & $0.28(1)$ & $0.034(6)$ & $0.039(9)$ & $-0.070(7)$ \\
\hline $\mathrm{Cl}(2)$ & $0.33(2)$ & $0.119(7)$ & $0.20(1)$ & $0.043(9)$ & $0.13(1)$ & $0.043(7)$ \\
\hline $\mathrm{P}(1)$ & $0.038(1)$ & $0.0308(9)$ & $0.0336(10)$ & $-0.0009(8)$ & $0.0115(9)$ & $0.0030(8)$ \\
\hline$P(2)$ & $0.037(1)$ & $0.0329(9)$ & $0.0298(9)$ & $-0.0018(8)$ & $0.0080(9)$ & $0.0017(8)$ \\
\hline$P(3)$ & $0.035(1)$ & $0.0366(10)$ & $0.0287(9)$ & $-0.0025(8)$ & $0.0055(8)$ & $-0.0009(8)$ \\
\hline $\mathrm{O}(1)$ & $0.077(5)$ & $0.104(6)$ & $0.087(5)$ & $0.016(4)$ & $0.017(5)$ & $-0.003(5)$ \\
\hline $\mathrm{N}(1)$ & $0.044(1)$ & $0.037(2)$ & $0.014(3)$ & $-0.027(2)$ & $0.002(3)$ & $-0.008(2)$ \\
\hline $\mathrm{N}(2)$ & $0.084(5)$ & $0.037(2)$ & $0.037(4)$ & $-0.003(4)$ & $0.019(4)$ & $-0.003(3)$ \\
\hline$C(1)$ & $0.056(5)$ & $0.039(3)$ & $0.034(4)$ & $0.001(4)$ & $0.018(4)$ & $-0.002(3)$ \\
\hline$C(2)$ & $0.042(4)$ & $0.033(2)$ & $0.038(4)$ & $0.003(3)$ & $0.009(3)$ & $-0.002(3)$ \\
\hline$C(3)$ & $0.053(5)$ & $0.032(3)$ & $0.027(4)$ & $0.006(3)$ & $0.013(4)$ & $0.008(3)$ \\
\hline$C(11)$ & $0.145(7)$ & $0.035(3)$ & $0.032(5)$ & $-0.001(4)$ & $0.031(6)$ & $-0.006(4)$ \\
\hline$C(12)$ & $0.152(9)$ & $0.063(5)$ & $0.051(6)$ & $0.034(6)$ & $0.026(7)$ & $0.003(5)$ \\
\hline$C(13)$ & $0.20(1)$ & $0.069(6)$ & $0.065(8)$ & $0.058(8)$ & $0.041(9)$ & $-0.001(6)$ \\
\hline$C(14)$ & $0.24(2)$ & $0.065(8)$ & $0.052(8)$ & $0.013(8)$ & $0.03(1)$ & $0.002(6)$ \\
\hline$C(15)$ & $0.21(1)$ & $0.068(5)$ & $0.079(9)$ & $-0.058(8)$ & $0.04(1)$ & $-0.022(7)$ \\
\hline$C(16)$ & $0.157(9)$ & $0.069(5)$ & $0.048(6)$ & $-0.038(6)$ & $0.006(8)$ & $-0.001(5)$ \\
\hline$C(17)$ & $0.12(1)$ & $0.10(1)$ & $0.16(1)$ & $0.045(7)$ & $0.04(1)$ & $0.012(10)$ \\
\hline$C(18)$ & $0.137(10)$ & $0.11(1)$ & $0.18(2)$ & $-0.077(8)$ & $0.06(1)$ & $-0.03(1)$ \\
\hline$C(111)$ & $0.056(4)$ & $0.031(2)$ & $0.032(4)$ & $0.004(3)$ & $0.012(4)$ & $0.002(3)$ \\
\hline$C(112)$ & $0.061(5)$ & $0.041(3)$ & $0.070(6)$ & $-0.006(4)$ & $0.020(5)$ & $0.008(4)$ \\
\hline$C(113)$ & $0.072(5)$ & $0.041(3)$ & $0.086(7)$ & $-0.019(4)$ & $0.016(6)$ & $-0.003(5)$ \\
\hline$C(114)$ & $0.088(5)$ & $0.026(4)$ & $0.070(6)$ & $-0.002(3)$ & $0.029(6)$ & $0.003(4)$ \\
\hline$C(115)$ & $0.073(5)$ & $0.041(3)$ & $0.073(6)$ & $0.014(4)$ & $0.009(5)$ & $0.002(4)$ \\
\hline$C(116)$ & $0.056(5)$ & $0.041(3)$ & $0.064(6)$ & $0.010(3)$ & $0.011(5)$ & $0.011(4)$ \\
\hline$C(121)$ & $0.041(3)$ & $0.030(4)$ & $0.049(3)$ & $-0.001(3)$ & $0.020(3)$ & $0.006(3)$ \\
\hline$C(122)$ & $0.067(5)$ & $0.055(5)$ & $0.054(4)$ & $-0.006(4)$ & $0.035(5)$ & $-0.010(4)$ \\
\hline$C(123)$ & $0.064(5)$ & $0.075(6)$ & $0.090(7)$ & $-0.006(5)$ & $0.052(4)$ & $-0.003(5)$ \\
\hline$C(124)$ & $0.065(6)$ & $0.076(6)$ & $0.106(6)$ & $0.012(5)$ & $0.055(5)$ & $0.013(6)$ \\
\hline$C(125)$ & $0.040(5)$ & $0.097(8)$ & $0.103(6)$ & $0.000(5)$ & $0.032(6)$ & $0.001(7)$ \\
\hline$C(126)$ & $0.047(4)$ & $0.065(5)$ & $0.051(5)$ & $0.001(4)$ & $0.015(3)$ & $0.000(4)$ \\
\hline$C(211)$ & $0.038(4)$ & $0.039(4)$ & $0.033(3)$ & $-0.010(2)$ & $0.009(3)$ & $-0.008(3)$ \\
\hline$C(212)$ & $0.062(5)$ & $0.046(4)$ & $0.038(4)$ & $-0.011(4)$ & $0.017(4)$ & $0.000(3)$ \\
\hline$C(213)$ & $0.070(6)$ & $0.062(5)$ & $0.067(6)$ & $-0.015(4)$ & $0.046(5)$ & $-0.023(4)$ \\
\hline$C(214)$ & $0.071(7)$ & $0.070(6)$ & $0.084(6)$ & $-0.003(4)$ & $0.041(6)$ & $-0.023(5)$ \\
\hline$C(215)$ & $0.055(6)$ & $0.080(7)$ & $0.075(5)$ & $0.021(5)$ & $0.012(5)$ & $-0.023(5)$ \\
\hline$C(216)$ & $0.044(5)$ & $0.046(4)$ & $0.052(5)$ & $-0.003(3)$ & $-0.003(4)$ & $-0.008(3)$ \\
\hline$C(311)$ & $0.039(4)$ & $0.045(4)$ & $0.034(3)$ & $-0.009(3)$ & $0.011(3)$ & $-0.005(3)$ \\
\hline$C(312)$ & $0.047(5)$ & $0.042(4)$ & $0.062(5)$ & $-0.009(3)$ & $0.013(4)$ & $-0.001(4)$ \\
\hline$C(313)$ & $0.078(7)$ & $0.047(5)$ & $0.099(6)$ & $-0.007(5)$ & $0.062(6)$ & $-0.029(5)$ \\
\hline$C(314)$ & $0.101(8)$ & $0.096(8)$ & $0.089(5)$ & $-0.039(5)$ & $0.070(6)$ & $-0.058(6)$ \\
\hline$C(315)$ & $0.113(9)$ & $0.122(10)$ & $0.046(6)$ & $-0.012(6)$ & $0.039(6)$ & $-0.034(6)$ \\
\hline$C(316)$ & $0.058(6)$ & $0.076(6)$ & $0.037(3)$ & $-0.011(5)$ & $0.001(4)$ & $0.004(4)$ \\
\hline$C(321)$ & $0.038(2)$ & $0.051(3)$ & $0.023(4)$ & $0.002(3)$ & $0.001(3)$ & $0.009(3)$ \\
\hline$C(322)$ & $0.034(4)$ & $0.064(5)$ & $0.104(8)$ & $-0.004(4)$ & $0.006(5)$ & $0.034(6)$ \\
\hline$C(323)$ & $0.033(4)$ & $0.075(6)$ & $0.14(1)$ & $-0.013(4)$ & $0.019(6)$ & $0.016(6)$ \\
\hline$C(324)$ & $0.031(5)$ & $0.086(5)$ & $0.062(6)$ & $0.000(3)$ & $0.002(4)$ & $0.011(5)$ \\
\hline$C(325)$ & $0.041(3)$ & $0.068(5)$ & $0.061(6)$ & $0.015(3)$ & $0.005(4)$ & $0.014(5)$ \\
\hline
\end{tabular}


$\mathrm{C}(326)$ $0.040(3)$ $0.046(4)$ $0.051(5)$ $0.004(3)$ $0.007(4)$ $-0.007(3)$

$\mathrm{a}$ Estmated standard deviations are given in parentheses.

$\mathrm{b}$ Parameters have the form $\exp \left(-2 \pi^{2}\left(U_{11} h^{2} a^{* 2}+\cdots+2 U_{12} h k a^{*} b^{*} \cos \gamma+\cdots\right)\right)$.

Table S8-4. Bond Lengths for Non-hydrogen Atoms of [ $\mathrm{Pt}_{3}(\mu$-dpmp) 2 ( $\mu$ $\left.\mathrm{NO})_{2}(\mathrm{XylNC})_{2}\right]\left(\mathrm{BF}_{4}\right)_{4} \cdot \mathrm{CH}_{2} \mathrm{Cl}_{2} \cdot 3 \mathrm{H}_{2} \mathrm{O}\left(\mathbf{1 0 a} \cdot \mathrm{CH}_{2} \mathrm{Cl}_{2} \cdot 3 \mathrm{H}_{2} \mathrm{O}\right)^{\mathrm{a}}$

\begin{tabular}{|c|c|c|c|c|c|}
\hline atom & atom & distance & atom & atom & distance \\
\hline$\overline{\mathrm{Pt}(1)}$ & $\mathrm{P}(1)$ & $2.358(2)$ & $\mathrm{C}(311)$ & $C(312)$ & $1.41(1)$ \\
\hline $\operatorname{Pt}(1)$ & $\mathrm{P}(3)$ & $2.343(2)$ & $C(311)$ & $C(316)$ & 1.41(1) \\
\hline $\operatorname{Pt}(1)$ & $\mathrm{N}(1)$ & $2.020(6)$ & $C(312)$ & $C(313)$ & $1.37(2)$ \\
\hline $\operatorname{Pt}(1)$ & $C(1)$ & $1.923(8)$ & $C(313)$ & $C(314)$ & $1.37(2)$ \\
\hline $\operatorname{Pt}(2)$ & $\mathrm{P}(2)$ & $2.321(2)$ & $C(314)$ & $C(315)$ & $1.44(2)$ \\
\hline $\operatorname{Pt}(2)$ & $\mathrm{P}(2)^{*}$ & $2.321(2)$ & $C(315)$ & $C(316)$ & $1.37(2)$ \\
\hline $\mathrm{Pt}(2)$ & $\mathrm{N}(1)$ & $2.019(6)$ & $C(321)$ & $C(322)$ & $1.37(1)$ \\
\hline $\operatorname{Pt}(2)$ & $\mathrm{N}(1)^{*}$ & $2.019(6)$ & $C(321)$ & $C(326)$ & $1.38(1)$ \\
\hline $\mathrm{P}(1)$ & $C(2)$ & $1.818(7)$ & $C(322)$ & $C(323)$ & 1.37(1) \\
\hline $\mathrm{P}(1)$ & $C(111)$ & $1.818(7)$ & $C(323)$ & $C(324)$ & $1.38(1)$ \\
\hline $\mathrm{P}(1)$ & $C(121)$ & 1.793(9) & $C(324)$ & $C(325)$ & 1.37(1) \\
\hline $\mathrm{P}(2)$ & $C(2)$ & $1.825(7)$ & $C(325)$ & $C(326)$ & $1.39(1)$ \\
\hline $\mathrm{P}(2)$ & $C(3)^{*}$ & $1.797(7)$ & $C(406)$ & $C(407)$ & $1.65(5)$ \\
\hline $\mathrm{P}(2)$ & $C(211)$ & $1.821(9)$ & $\mathrm{B}(1)$ & $\mathrm{F}(1)$ & $1.360(8)$ \\
\hline $\mathrm{P}(3)$ & $C(3)$ & $1.842(7)$ & $\mathrm{B}(1)$ & $\mathrm{F}(2)$ & $1.36(1)$ \\
\hline$P(3)$ & $C(311)$ & $1.785(9)$ & $\mathrm{B}(1)$ & $\mathrm{F}(3)$ & $1.360(8)$ \\
\hline$P(3)$ & $C(321)$ & $1.811(7)$ & $\mathrm{B}(1)$ & $\mathrm{F}(4)$ & $1.360(9)$ \\
\hline $\mathrm{O}(1)$ & $\mathrm{N}(1)$ & $1.09(1)$ & $\mathrm{B}(2)$ & $\mathrm{F}(5)$ & $1.36(1)$ \\
\hline$N(2)$ & $C(1)$ & $1.14(1)$ & $\mathrm{B}(2)$ & $\mathrm{F}(6)$ & $1.36(2)$ \\
\hline$N(2)$ & $C(11)$ & $1.43(1)$ & $B(2)$ & $\mathrm{F}(7)$ & $1.36(1)$ \\
\hline$C(11)$ & $C(12)$ & $1.41(2)$ & $\mathrm{B}(2)$ & $\mathrm{F}(8)$ & $1.36(1)$ \\
\hline$C(11)$ & $C(16)$ & $1.32(2)$ & $\mathrm{Cl}(1)$ & $C(406)$ & $1.52(4)$ \\
\hline$C(12)$ & $C(13)$ & $1.41(2)$ & $\mathrm{Cl}(1)$ & $C(407)$ & $2.00(4)$ \\
\hline$C(12)$ & $C(17)$ & $1.32(2)$ & $\mathrm{Cl}(2)$ & $C(406)$ & $1.61(3)$ \\
\hline$C(13)$ & $C(14)$ & $1.36(3)$ & $\mathrm{Cl}(2)$ & $C(407)$ & $1.46(5)$ \\
\hline$C(14)$ & $C(15)$ & $1.42(3)$ & & & \\
\hline$C(15)$ & $C(16)$ & $1.48(2)$ & & & \\
\hline$C(16)$ & $C(18)$ & $1.41(2)$ & & & \\
\hline$C(111)$ & $C(112)$ & $1.41(1)$ & & & \\
\hline$C(111)$ & $C(116)$ & $1.40(1)$ & & & \\
\hline$C(112)$ & $C(113)$ & $1.40(1)$ & & & \\
\hline C(113) & $C(114)$ & $1.32(1)$ & & & \\
\hline$C(114)$ & $C(115)$ & $1.37(1)$ & & & \\
\hline$C(115)$ & $C(116)$ & $1.36(1)$ & & & \\
\hline$C(121)$ & $C(122)$ & $1.41(1)$ & & & \\
\hline$C(121)$ & $C(126)$ & $1.37(1)$ & & & \\
\hline$C(122)$ & $C(123)$ & $1.38(2)$ & & & \\
\hline$C(123)$ & $C(124)$ & $1.33(1)$ & & & \\
\hline$C(124)$ & $C(125)$ & $1.35(2)$ & & & \\
\hline$C(125)$ & $C(126)$ & $1.42(2)$ & & & \\
\hline$C(211)$ & $C(212)$ & $1.38(1)$ & & & \\
\hline$C(211)$ & $C(216)$ & $1.39(1)$ & & & \\
\hline$C(212)$ & $C(213)$ & $1.38(1)$ & & & \\
\hline$C(213)$ & $C(214)$ & $1.35(1)$ & & & \\
\hline$C(214)$ & $C(215)$ & $1.40(2)$ & & & \\
\hline$C(215)$ & $C(216)$ & $1.38(2)$ & & & \\
\hline
\end{tabular}

a Estimated standard deviations are given in parentheses. 
Table S8-5. Bond Angles for Non-hydrogen Atoms of $\left[\mathrm{Pt}_{3}(\mu-\mathrm{dpmp})_{2}(\mu-\right.$ $\mathrm{NO})_{2}(\mathrm{XylNC})_{2} \mathrm{I}\left(\mathrm{BF}_{4}\right)_{4} \cdot \mathrm{CH}_{2} \mathrm{Cl}_{2} \cdot 3 \mathrm{H}_{2} \mathrm{O}\left(\mathbf{1 0 a} \cdot \mathrm{CH}_{2} \mathrm{Cl}_{2} \cdot 3 \mathrm{H}_{2} \mathrm{O}\right)^{\mathrm{a}}$

\begin{tabular}{|c|c|c|c|c|c|c|c|}
\hline atom & atom & atom & angle & atom & atom & atom & angle \\
\hline$\overline{\mathrm{P}(1)}$ & $\operatorname{Pt}(1)$ & $\mathrm{P}(3)$ & $171.47(7)$ & $\operatorname{Pt}(1)$ & $\mathrm{P}(3)$ & $C(3)$ & $112.2(2)$ \\
\hline$P(1)$ & $\operatorname{Pt}(1)$ & $\mathrm{N}(1)$ & $86.6(2)$ & $\operatorname{Pt}(1)$ & $P(3)$ & $C(311)$ & $118.1(2)$ \\
\hline $\mathrm{P}(1)$ & $\operatorname{Pt}(1)$ & $C(1)$ & $90.4(2)$ & $\operatorname{Pt}(1)$ & $\mathrm{P}(3)$ & $C(321)$ & $107.3(3)$ \\
\hline$P(3)$ & $\operatorname{Pt}(1)$ & $\mathrm{N}(1)$ & $87.0(2)$ & $C(3)$ & $P(3)$ & $C(311)$ & $105.7(4)$ \\
\hline$P(3)$ & $\operatorname{Pt}(1)$ & $C(1)$ & $96.3(2)$ & $C(3)$ & $\mathrm{P}(3)$ & $C(321)$ & $104.9(3)$ \\
\hline $\mathrm{N}(1)$ & $\operatorname{Pt}(1)$ & $C(1)$ & $176.1(2)$ & $C(311)$ & $\mathrm{P}(3)$ & $C(321)$ & $107.9(3)$ \\
\hline$P(2)$ & $\operatorname{Pt}(2)$ & $\mathrm{P}(2)^{*}$ & 180.00 & $\operatorname{Pt}(1)$ & $\mathrm{N}(1)$ & $\operatorname{Pt}(2)$ & 100.1(3) \\
\hline $\mathrm{P}(2)$ & $\operatorname{Pt}(2)$ & $\mathrm{N}(1)$ & $87.1(2)$ & $\operatorname{Pt}(1)$ & $\mathrm{N}(1)$ & $\mathrm{O}(1)$ & $125.9(6)$ \\
\hline $\mathrm{P}(2)$ & $\operatorname{Pt}(2)$ & $\mathrm{N}(1)^{*}$ & $92.9(2)$ & $\operatorname{Pt}(2)$ & $\mathrm{N}(1)$ & $\mathrm{O}(1)$ & $133.2(6)$ \\
\hline $\mathrm{P}(2)^{*}$ & $\operatorname{Pt}(2)$ & $\mathrm{N}(1)$ & $92.9(2)$ & $C(1)$ & $\mathrm{N}(2)$ & $C(11)$ & $172.7(7)$ \\
\hline $\mathrm{P}(2)$ & $\operatorname{Pt}(2)$ & $\mathrm{N}(1)^{*}$ & $87.1(2)$ & $\operatorname{Pt}(1)$ & $C(1)$ & $\mathrm{N}(2)$ & $175.7(7)$ \\
\hline $\mathrm{N}(1)$ & $\operatorname{Pt}(2)$ & $\mathrm{N}(1)^{*}$ & 180.00 & $\mathrm{P}(1)$ & $C(2)$ & $P(2)$ & $118.3(4)$ \\
\hline$C(406)$ & $\mathrm{Cl}(1)$ & $C(407)$ & $54(2)$ & $\mathrm{P}(2)$ & $C(3)$ & $P(3)$ & $114.4(4)$ \\
\hline$C(406)$ & $\mathrm{Cl}(2)$ & $C(407)$ & $65(2)$ & $\mathrm{N}(2)$ & $C(11)$ & $C(12)$ & $117.4(9)$ \\
\hline $\operatorname{Pt}(1)$ & $P(1)$ & $C(2)$ & $113.3(3)$ & $\mathrm{N}(2)$ & $C(11)$ & $C(16)$ & $116(1)$ \\
\hline $\operatorname{Pt}(1)$ & $\mathrm{P}(1)$ & $C(111)$ & $114.2(3)$ & $C(12)$ & $C(11)$ & $C(16)$ & 127(1) \\
\hline $\operatorname{Pt}(1)$ & $P(1)$ & $C(121)$ & $109.2(2)$ & $C(11)$ & $C(12)$ & $C(13)$ & 119(1) \\
\hline$C(2)$ & $P(1)$ & $C(111)$ & $105.1(3)$ & $C(11)$ & $C(12)$ & $C(17)$ & 121(1) \\
\hline$C(2)$ & $P(1)$ & $C(121)$ & $107.9(4)$ & $C(13)$ & $C(12)$ & $C(17)$ & $120(2)$ \\
\hline$C(111)$ & $\mathrm{P}(1)$ & $C(121)$ & $106.8(4)$ & $C(12)$ & $C(13)$ & $C(14)$ & $116(2)$ \\
\hline $\operatorname{Pt}(2)$ & $P(2)$ & $C(2)$ & $108.9(3)$ & $C(13)$ & $C(14)$ & $C(15)$ & $127(1)$ \\
\hline $\operatorname{Pt}(2)$ & $\mathrm{P}(2)$ & $C(3)$ & 110.7(3) & $C(14)$ & $C(15)$ & $C(16)$ & $116(1)$ \\
\hline $\operatorname{Pt}(2)$ & $P(2)$ & $C(211)$ & $118.2(2)$ & $C(11)$ & $C(16)$ & $C(15)$ & $116(1)$ \\
\hline$C(2)$ & $P(2)$ & $C(3)$ & $105.8(3)$ & $C(11)$ & $C(16)$ & $C(18)$ & $125(1)$ \\
\hline$C(2)$ & $P(2)$ & $C(211)$ & $107.8(4)$ & $C(15)$ & $C(16)$ & $C(18)$ & 119(1) \\
\hline$C(3)$ & $\mathrm{P}(2)$ & $C(211)$ & $104.7(3)$ & $\mathrm{P}(1)$ & $C(111)$ & $C(112)$ & $117.6(6)$ \\
\hline$P(1)$ & $C(111)$ & $C(116)$ & $121.9(6)$ & $C(311)$ & $C(312)$ & $C(313)$ & $122.0(8)$ \\
\hline$C(112)$ & $C(111)$ & $C(116)$ & $120.3(7)$ & $C(312)$ & $C(313)$ & $C(314)$ & $120.2(9)$ \\
\hline$C(111)$ & $C(112)$ & $C(113)$ & $117.8(8)$ & $C(313)$ & $C(314)$ & $C(315)$ & $119(1)$ \\
\hline$C(112)$ & $C(113)$ & $C(114)$ & $120.7(9)$ & $C(314)$ & $C(315)$ & $C(316)$ & $119.8(9)$ \\
\hline$C(113)$ & $C(114)$ & $C(115)$ & $121.3(8)$ & $C(311)$ & $C(316)$ & $C(315)$ & $120.8(9)$ \\
\hline$C(114)$ & $C(115)$ & $C(116)$ & $121.8(9)$ & $P(3)$ & $C(321)$ & $C(322)$ & $117.4(6)$ \\
\hline$C(111)$ & $C(116)$ & $C(115)$ & $118.0(8)$ & $P(3)$ & $C(321)$ & $C(326)$ & $124.0(6)$ \\
\hline $\mathrm{P}(1)$ & $C(121)$ & $C(122)$ & $121.9(6)$ & $C(322)$ & $C(321)$ & $C(326)$ & $118.5(7)$ \\
\hline$P(1)$ & $C(121)$ & $C(126)$ & $119.5(7)$ & $C(321)$ & $C(322)$ & $C(323)$ & $121.6(9)$ \\
\hline$C(122)$ & $C(121)$ & $C(126)$ & $118.5(8)$ & $C(322)$ & $C(323)$ & $C(324)$ & $120.5(9)$ \\
\hline$C(121)$ & $C(122)$ & $C(123)$ & $120.4(8)$ & $C(323)$ & $C(324)$ & $C(325)$ & $118.2(8)$ \\
\hline$C(122)$ & $C(123)$ & $C(124)$ & 121(1) & $C(324)$ & $C(325)$ & $C(326)$ & $121.3(8)$ \\
\hline$C(123)$ & $C(124)$ & $C(125)$ & 121(1) & $C(321)$ & $C(326)$ & $C(325)$ & $119.8(7)$ \\
\hline$C(124)$ & $C(125)$ & $C(126)$ & $120.8(9)$ & $\mathrm{Cl}(1)$ & $C(406)$ & $\mathrm{Cl}(2)$ & $127(2)$ \\
\hline$C(121)$ & $C(126)$ & $C(125)$ & $118.6(9)$ & $\mathrm{Cl}(1)$ & $C(406)$ & $C(407)$ & $78(2)$ \\
\hline $\mathrm{P}(2)$ & $C(211)$ & $C(212)$ & 119.1(6) & $\mathrm{Cl}(2)$ & $C(406)$ & $C(407)$ & $53(2)$ \\
\hline $\mathrm{P}(2)$ & $C(211)$ & $C(216)$ & $120.6(7)$ & $\mathrm{Cl}(1)$ & $C(407)$ & $\mathrm{Cl}(2)$ & 107(2) \\
\hline$C(212)$ & $C(211)$ & $C(216)$ & $120.2(8)$ & $\mathrm{Cl}(1)$ & $C(407)$ & $C(406)$ & $48(1)$ \\
\hline$C(211)$ & $C(212)$ & $C(213)$ & $118.7(7)$ & $\mathrm{Cl}(2)$ & $C(407)$ & $C(406)$ & $62(2)$ \\
\hline$C(212)$ & $C(213)$ & $C(214)$ & $122(1)$ & $\mathrm{F}(1)$ & $\mathrm{B}(1)$ & $F(2)$ & $109.5(6)$ \\
\hline$C(213)$ & $C(214)$ & $C(215)$ & $120(1)$ & $\mathrm{F}(1)$ & $\mathrm{B}(1)$ & $F(4)$ & $109.5(6)$ \\
\hline$C(214)$ & $C(215)$ & $C(216)$ & $118.8(9)$ & $\mathrm{F}(1)$ & $\mathrm{B}(1)$ & $F(3)$ & $109.5(7)$ \\
\hline$C(211)$ & $C(216)$ & $C(215)$ & $120.2(9)$ & $F(2)$ & $\mathrm{B}(1)$ & $\mathrm{F}(4)$ & $109.4(7)$ \\
\hline$P(3)$ & $C(311)$ & $C(312)$ & $121.7(6)$ & $F(2)$ & $\mathrm{B}(1)$ & $F(3)$ & $109.4(7)$ \\
\hline $\mathrm{P}(3)$ & $C(311)$ & $C(316)$ & $120.5(6)$ & $\mathrm{F}(4)$ & $\mathrm{B}(1)$ & $F(3)$ & $109.4(6)$ \\
\hline$C(312)$ & $C(311)$ & $C(316)$ & $117.8(8)$ & $F(5)$ & $\mathrm{B}(2)$ & $F(6)$ & $110(1)$ \\
\hline
\end{tabular}




$\begin{array}{llll}\mathrm{F}(5) & \mathrm{B}(2) & \mathrm{F}(7) & 110(1) \\ \mathrm{F}(5) & \mathrm{B}(2) & \mathrm{F}(8) & 110(1) \\ \mathrm{F}(6) & \mathrm{B}(2) & \mathrm{F}(7) & 109(1) \\ \mathrm{F}(6) & \mathrm{B}(2) & \mathrm{F}(8) & 109(1) \\ \mathrm{F}(7) & \mathrm{B}(2) & \mathrm{F}(8) & 109(1)\end{array}$

a Estimated deviations are given in parentheses. 
Table S9-1. Crystallographic and Experimental Data for $\left[\mathrm{Pt}_{3}(\mu-\mathrm{dpmp})_{2}\left(\mu-\mathrm{C}_{12} \mathrm{~N}_{8}\right)\left(\mathrm{XylNC}_{2}\right)_{-}\right.$ $\left(\mathrm{PF}_{6}\right)_{2} \cdot 2\left(\mathrm{CH}_{3}\right)_{2} \mathrm{CO}\left(\mathbf{1 1 a} \cdot\left(\mathrm{CH}_{3}\right)_{2} \mathrm{CO}\right)$

\begin{tabular}{|c|c|}
\hline formula & $\mathrm{C}_{97} \mathrm{H}_{82} \mathrm{~N}_{10} \mathrm{OP}_{8} \mathrm{~F}_{12} \mathrm{Pt}_{3}$ \\
\hline formula weight & 2464.82 \\
\hline color & pale yellow \\
\hline size, $\mathrm{mm}$ & $0.45 \times 0.20 \times 0.20$ \\
\hline cryst. system & monoclinic \\
\hline space group & $P 21 / c$ (No. 14) \\
\hline no. refs used for cell determination & $25\left(20<2 \theta<30^{\circ}\right)$ \\
\hline lattice const. & $\begin{array}{l}a=13.872(4) \AA \\
b=53.155(12) \AA \\
c=15.130(4) \AA \\
\beta=97.90(2)^{\circ} \\
V=11050(4) \AA^{3}\end{array}$ \\
\hline Z & 4 \\
\hline$D_{\text {calc }}, \mathrm{g} \mathrm{cm}^{-3}$ & 1.481 \\
\hline$\mu(\mathrm{Mo} K \alpha), \mathrm{cm}^{-1}$ & 39.55 (trans. $0.73 \sim 1.00$ ) \\
\hline \multicolumn{2}{|c|}{ An absorption correction by the $\psi$-scan method was applied. } \\
\hline diffractometer & Rigaku AFC7R (50 kV 200 mA) \\
\hline radiation & $\operatorname{Mo} K \alpha(1=0.71069 \AA)$ \\
\hline monochromator & graphite \\
\hline temp, ${ }^{\circ} \mathrm{C}$ & -118 \\
\hline $2 \theta$ range, ${ }^{\circ}$ & $4<2 \theta<45$ \\
\hline$h, k, l$ range & $+h,+k, \pm l$ \\
\hline scan method & $\omega$ \\
\hline scan speed, ${ }^{\circ} \min ^{-1}$ & 8 \\
\hline scan width & $1.21+0.30 \tan \theta$ \\
\hline std. reflections & 3 every 150 refs \\
\hline$F_{000}$ & 4816.00 \\
\hline solution & Patterson Methods (DIRDIF94) \\
\hline \multirow[t]{2}{*}{ refinement } & full-matrix least-squares \\
\hline & $\begin{array}{l}\text { All non-hydrogen atoms except phenyl } \\
\text { groups and lattice acetones were refined } \\
\text { with aniotropic temperature factors. }\end{array}$ \\
\hline hydrogen atoms & $\begin{array}{l}\text { All C-H hydrogen atoms except those of } \\
\text { acetones were calculated at ideal positions } \\
\text { with } \mathrm{C}-\mathrm{H}=0.95 \AA \text {, and were not refined. }\end{array}$ \\
\hline function minimized & $\Sigma w\left(F o^{2}-F c^{2}\right)^{2}$ \\
\hline weighting scheme & $\begin{array}{l}w=\left[\sigma^{2}\left(F o^{2}\right)+(0.0889 P)^{2}+230.9415 P\right]^{-1} \\
P=\left(F o^{2}+2 F c^{2}\right) / 3\end{array}$ \\
\hline anomalous dispersion & all non-hydrogen atoms \\
\hline no. unique reflections & 14381 \\
\hline no. obsd. unique reflections & $10081(I>2 \sigma(I))$ \\
\hline no. variables & 877 \\
\hline data / param ratio & 11.49 \\
\hline$R_{1}{ }^{\mathrm{a}}$ & 0.068 \\
\hline$w R_{2} b$ & 0.227 \\
\hline GOF & 1.39 \\
\hline$\rho_{\max }, \min , \mathrm{e}^{-3}$ & $1.69,-1.54$ \\
\hline
\end{tabular}

a $R=\Sigma|| F_{\mathrm{O}}|-| F_{\mathrm{C}}|| / \Sigma\left|F_{\mathrm{O}}\right|$ for observed data.

$\mathrm{b}_{R_{\mathrm{W}}}=\left[\Sigma w\left(F_{\mathrm{O}}^{2}-F_{\mathrm{C}}^{2}\right)^{2} / \Sigma w\left(F_{\mathrm{O}}^{2}\right)^{2}\right]^{1 / 2}$ for all data. 
Table S9-2. Final Positional Parameters and $B_{\text {eq }}$ for $\left[\mathrm{Pt}_{3}(\mu-\mathrm{dpmp})_{2}\left(\mu-\mathrm{C}_{12} \mathrm{~N}_{8}\right)\left(\mathrm{XylNC}_{2}\right)_{-}\right.$ $\left(\mathrm{PF}_{6}\right)_{2} \cdot 2\left(\mathrm{CH}_{3}\right)_{2} \mathrm{CO}\left(\mathbf{1 1 a} \cdot\left(\mathrm{CH}_{3}\right)_{2} \mathrm{CO}\right)^{\mathrm{a}, \mathrm{b}, \mathrm{c}}$

\begin{tabular}{|c|c|c|c|c|}
\hline atom & $x$ & $y$ & $z$ & $B_{\mathrm{eq}}$ \\
\hline$\overline{P t(1)}$ & $0.06399(5)$ & $0.17120(1)$ & $0.29884(4)$ & $2.74(2)$ \\
\hline $\operatorname{Pt}(2)$ & $0.14162(5)$ & $0.12653(1)$ & $0.27775(4)$ & $2.57(2)$ \\
\hline $\operatorname{Pt}(3)$ & $0.09995(5)$ & $0.06725(1)$ & $0.25389(4)$ & $2.73(2)$ \\
\hline $\mathrm{P}(1)$ & $0.0356(4)$ & $0.16292(8)$ & $0.4430(3)$ & $3.28(9)$ \\
\hline $\mathrm{P}(2)$ & $0.1786(3)$ & $0.12313(8)$ & $0.4277(3)$ & $2.77(8)$ \\
\hline$P(3)$ & $0.1263(3)$ & $0.06620(7)$ & $0.4100(3)$ & $2.82(8)$ \\
\hline $\mathrm{P}(4)$ & $0.1052(3)$ & $0.18308(7)$ & $0.1608(3)$ & $2.90(8)$ \\
\hline$P(5)$ & $0.0761(3)$ & $0.12888(8)$ & $0.1315(3)$ & $2.75(8)$ \\
\hline$P(6)$ & $0.0742(3)$ & $0.07226(8)$ & $0.0982(3)$ & $2.97(8)$ \\
\hline $\mathrm{P}(11)$ & $0.5627(5)$ & $0.2420(1)$ & $0.4986(6)$ & $7.0(2)$ \\
\hline$P(12)$ & $0.1039(8)$ & $0.1200(1)$ & $0.7879(5)$ & $8.7(2)$ \\
\hline $\mathrm{F}(11)$ & $0.490(2)$ & $0.2530(5)$ & $0.434(2)$ & $20(1)$ \\
\hline $\mathrm{F}(12)$ & $0.561(1)$ & $0.2677(4)$ & $0.540(2)$ & $17(1)$ \\
\hline $\mathrm{F}(13)$ & $0.656(1)$ & $0.2493(4)$ & $0.458(2)$ & $15.6(10)$ \\
\hline $\mathrm{F}(14)$ & $0.466(2)$ & $0.2344(4)$ & $0.530(2)$ & $19(1)$ \\
\hline$F(15)$ & $0.627(3)$ & $0.2292(7)$ & $0.569(2)$ & $26(1)$ \\
\hline$F(16)$ & $0.560(1)$ & $0.2164(3)$ & $0.447(2)$ & $12.6(7)$ \\
\hline $\mathrm{F}(21)$ & $0.185(1)$ & $0.1395(3)$ & $0.7711(10)$ & $9.8(5)$ \\
\hline $\mathrm{F}(22)$ & $0.025(2)$ & $0.1322(3)$ & $0.710(1)$ & $11.8(6)$ \\
\hline $\mathrm{F}(23)$ & $0.065(2)$ & $0.1369(4)$ & $0.860(1)$ & $14.0(7)$ \\
\hline $\mathrm{F}(24)$ & $0.176(2)$ & $0.1049(4)$ & $0.858(1)$ & $12.8(6)$ \\
\hline $\mathrm{F}(25)$ & $0.027(2)$ & $0.0997(3)$ & $0.807(1)$ & $13.0(7)$ \\
\hline$F(26)$ & $0.137(2)$ & $0.1033(3)$ & $0.712(1)$ & $10.7(5)$ \\
\hline $\mathrm{O}(701)^{\mathrm{d}}$ & $0.556(2)$ & $0.1420(4)$ & $0.191(1)$ & $3.4(6)$ \\
\hline $\mathrm{O}(702)^{\mathrm{d}}$ & $0.734(4)$ & $0.0915(10)$ & $0.723(3)$ & $12(2)$ \\
\hline $\mathrm{N}(1)$ & $-0.024(1)$ & $0.2246(3)$ & $0.3128(9)$ & $4.2(3)$ \\
\hline $\mathrm{N}(2)$ & $-0.049(1)$ & $0.0249(3)$ & $0.2517(8)$ & $3.4(3)$ \\
\hline $\mathrm{N}(11)$ & $0.216(1)$ & $0.0914(3)$ & $0.2558(9)$ & $3.3(3)$ \\
\hline $\mathrm{N}(21)$ & $0.246(1)$ & $0.0176(3)$ & $0.242(1)$ & $4.9(4)$ \\
\hline $\mathrm{N}(22)$ & $0.533(2)$ & $0.0453(4)$ & $0.401(1)$ & $6.7(5)$ \\
\hline $\mathrm{N}(23)$ & $0.511(2)$ & $0.0274(5)$ & $0.118(1)$ & $10.0(8)$ \\
\hline $\mathrm{N}(24)$ & $0.652(1)$ & $0.0939(4)$ & $0.275(1)$ & $6.6(5)$ \\
\hline $\mathrm{N}(25)$ & $0.436(2)$ & $0.0927(5)$ & $0.042(1)$ & $9.3(7)$ \\
\hline $\mathrm{N}(26)$ & $0.456(1)$ & $0.1084(3)$ & $0.428(1)$ & $5.1(4)$ \\
\hline $\mathrm{N}(27)$ & $0.334(1)$ & $0.1419(4)$ & $0.175(1)$ & $6.0(4)$ \\
\hline$C(1)$ & $0.010(1)$ & $0.2058(4)$ & $0.313(1)$ & 4.1(4) \\
\hline$C(2)$ & $0.004(1)$ & $0.0418(3)$ & $0.249(1)$ & $3.3(4)$ \\
\hline$C(3)$ & $0.073(1)$ & $0.1311(3)$ & $0.484(1)$ & $3.0(3)$ \\
\hline$C(4)$ & $0.208(1)$ & $0.0906(3)$ & $0.464(1)$ & $3.4(3)$ \\
\hline$C(5)$ & $0.114(1)$ & $0.1577(3)$ & $0.079(1)$ & $2.8(3)$ \\
\hline$C(6)$ & $0.115(1)$ & $0.1028(3)$ & $0.065(1)$ & $2.9(3)$ \\
\hline$C(11)$ & $0.301(1)$ & $0.0839(3)$ & $0.251(1)$ & $3.5(4)$ \\
\hline$C(12)$ & $0.336(1)$ & $0.0584(3)$ & $0.239(1)$ & $3.6(4)$ \\
\hline$C(13)$ & $0.447(1)$ & $0.0586(4)$ & $0.240(1)$ & $4.7(4)$ \\
\hline$C(14)$ & $0.468(2)$ & $0.0871(4)$ & $0.215(1)$ & 5.3(5) \\
\hline$C(15)$ & $0.388(1)$ & $0.1018(3)$ & $0.258(1)$ & $2.9(3)$ \\
\hline$C(21)$ & $0.282(1)$ & $0.0361(4)$ & $0.240(1)$ & $3.6(4)$ \\
\hline$C(22)$ & $0.499(1)$ & $0.0513(4)$ & $0.331(2)$ & $4.7(4)$ \\
\hline$C(23)$ & $0.480(2)$ & $0.0406(5)$ & $0.166(2)$ & $6.7(6)$ \\
\hline$C(24)$ & $0.575(2)$ & $0.0925(4)$ & $0.249(1)$ & $4.5(4)$ \\
\hline$C(25)$ & $0.451(1)$ & $0.0894(5)$ & $0.115(2)$ & $5.8(5)$ \\
\hline$C(26)$ & $0.431(2)$ & $0.1065(4)$ & $0.355(2)$ & $5.3(5)$ \\
\hline$C(27)$ & $0.361(1)$ & $0.1262(4)$ & $0.213(2)$ & $5.2(5)$ \\
\hline$C(31)$ & $-0.062(2)$ & $0.2500(3)$ & $0.307(1)$ & $5.4(6)$ \\
\hline
\end{tabular}




\begin{tabular}{|c|c|c|c|c|}
\hline$C(32)$ & $-0.163(2)$ & $0.2530(5)$ & $0.281(2)$ & $7.1(6)$ \\
\hline$C(33)$ & $-0.188(3)$ & $0.2774(9)$ & $0.274(2)$ & $12(1)$ \\
\hline$C(34)$ & $-0.106(4)$ & $0.2975(6)$ & $0.304(3)$ & $10(1)$ \\
\hline C(35) & $-0.025(3)$ & $0.2933(5)$ & $0.333(2)$ & $9.0(10)$ \\
\hline$C(36)$ & $0.004(2)$ & $0.2689(5)$ & $0.333(2)$ & $6.6(6)$ \\
\hline$C(37)$ & $-0.229(2)$ & $0.2319(5)$ & $0.260(2)$ & $7.4(6)$ \\
\hline$C(38)$ & $0.108(2)$ & $0.2625(4)$ & $0.358(2)$ & $7.3(7)$ \\
\hline$C(41)$ & $-0.110(1)$ & $0.0020(3)$ & $0.254(1)$ & $3.8(4)$ \\
\hline$C(42)$ & $-0.205(1)$ & $0.0050(3)$ & $0.264(1)$ & $3.8(4)$ \\
\hline$C(43)$ & $-0.257(2)$ & $-0.0173(4)$ & $0.268(1)$ & $4.9(5)$ \\
\hline$C(44)$ & $-0.219(2)$ & $-0.0392(4)$ & $0.256(2)$ & $5.4(5)$ \\
\hline$C(45)$ & $-0.123(2)$ & $-0.0415(3)$ & $0.243(1)$ & $5.4(6)$ \\
\hline$C(46)$ & $-0.061(2)$ & $-0.0210(3)$ & $0.241(1)$ & $4.3(4)$ \\
\hline$C(47)$ & $-0.251(1)$ & $0.0293(4)$ & $0.272(1)$ & $5.0(5)$ \\
\hline$C(48)$ & $0.045(2)$ & $-0.0218(3)$ & $0.229(1)$ & $4.8(5)$ \\
\hline$C(111)$ & $0.104(1)$ & $0.1839(3)$ & $0.524(1)$ & $2.8(4)$ \\
\hline$C(112)$ & $0.097(1)$ & $0.1807(4)$ & $0.614(1)$ & $4.7(5)$ \\
\hline$C(113)$ & $0.155(2)$ & $0.1957(4)$ & $0.678(1)$ & $5.7(6)$ \\
\hline$C(114)$ & $0.214(2)$ & $0.2137(4)$ & $0.652(1)$ & $5.5(6)$ \\
\hline$C(115)$ & $0.219(1)$ & $0.2165(4)$ & $0.559(1)$ & $4.3(5)$ \\
\hline$C(116)$ & $0.164(1)$ & $0.2017(4)$ & $0.496(1)$ & $4.2(5)$ \\
\hline$C(121)$ & $-0.092(1)$ & $0.1642(3)$ & $0.458(1)$ & $3.8(5)$ \\
\hline$C(122)$ & $-0.124(2)$ & $0.1834(5)$ & $0.511(2)$ & $6.9(7)$ \\
\hline$C(123)$ & $-0.230(2)$ & $0.1844(5)$ & $0.514(2)$ & $7.4(8)$ \\
\hline$C(124)$ & $-0.290(2)$ & $0.1680(4)$ & $0.474(2)$ & $6.2(7)$ \\
\hline$C(125)$ & $-0.257(2)$ & $0.1475(4)$ & $0.423(1)$ & $5.6(6)$ \\
\hline$C(126)$ & $-0.158(1)$ & $0.1477(4)$ & $0.417(1)$ & $4.7(5)$ \\
\hline$C(211)$ & $0.277(1)$ & $0.1423(3)$ & $0.484(1)$ & $2.7(4)$ \\
\hline$C(212)$ & $0.303(1)$ & $0.1410(4)$ & $0.575(1)$ & $4.4(5)$ \\
\hline$C(213)$ & $0.376(2)$ & $0.1566(4)$ & $0.614(1)$ & $5.5(6)$ \\
\hline$C(214)$ & $0.428(2)$ & $0.1717(4)$ & $0.566(1)$ & $5.6(6)$ \\
\hline$C(215)$ & $0.401(2)$ & $0.1734(4)$ & $0.474(1)$ & $5.3(6)$ \\
\hline$C(216)$ & $0.325(1)$ & $0.1577(3)$ & $0.433(1)$ & $3.5(4)$ \\
\hline$C(311)$ & $0.013(1)$ & $0.0702(3)$ & $0.459(1)$ & $3.2(4)$ \\
\hline$C(312)$ & $0.015(1)$ & $0.0684(3)$ & $0.552(1)$ & $3.9(5)$ \\
\hline$C(313)$ & $-0.066(1)$ & $0.0725(4)$ & $0.588(1)$ & $4.7(5)$ \\
\hline$C(314)$ & $-0.150(2)$ & $0.0780(4)$ & $0.539(1)$ & $5.6(6)$ \\
\hline$C(315)$ & $-0.157(2)$ & $0.0804(4)$ & $0.447(1)$ & $5.2(6)$ \\
\hline$C(316)$ & $-0.073(1)$ & $0.0759(3)$ & $0.406(1)$ & $3.5(4)$ \\
\hline$C(321)$ & $0.180(1)$ & $0.0368(3)$ & $0.4554(10)$ & $2.7(4)$ \\
\hline$C(322)$ & $0.118(1)$ & $0.0165(3)$ & $0.459(1)$ & $3.5(4)$ \\
\hline$C(323)$ & $0.159(1)$ & $-0.0074(4)$ & $0.484(1)$ & $4.8(5)$ \\
\hline$C(324)$ & $0.256(2)$ & $-0.0091(4)$ & $0.501(1)$ & $5.2(6)$ \\
\hline$C(325)$ & $0.321(2)$ & $0.0103(4)$ & $0.497(1)$ & $5.2(6)$ \\
\hline$C(326)$ & $0.278(1)$ & $0.0344(4)$ & $0.474(1)$ & $4.9(5)$ \\
\hline$C(411)$ & $0.018(1)$ & $0.2059(3)$ & $0.103(1)$ & $3.2(4)$ \\
\hline$C(412)$ & $-0.076(1)$ & $0.1981(3)$ & $0.072(1)$ & $3.7(4)$ \\
\hline$C(413)$ & $-0.142(2)$ & $0.2164(4)$ & $0.034(1)$ & $5.1(6)$ \\
\hline$C(414)$ & $-0.116(1)$ & $0.2416(4)$ & $0.034(1)$ & $4.6(5)$ \\
\hline$C(415)$ & $-0.024(1)$ & $0.2490(4)$ & $0.069(1)$ & $4.8(5)$ \\
\hline$C(416)$ & $0.043(1)$ & $0.2315(4)$ & $0.103(1)$ & $4.1(5)$ \\
\hline$C(421)$ & $0.219(1)$ & $0.1993(3)$ & $0.174(1)$ & $2.9(4)$ \\
\hline$C(422)$ & $0.263(1)$ & $0.2053(4)$ & $0.097(1)$ & $4.6(5)$ \\
\hline$C(423)$ & $0.351(2)$ & $0.2203(4)$ & $0.104(1)$ & $5.3(6)$ \\
\hline$C(424)$ & $0.385(2)$ & $0.2289(4)$ & $0.187(1)$ & $5.3(6)$ \\
\hline$C(425)$ & $0.349(2)$ & $0.2226(4)$ & $0.261(1)$ & $5.7(6)$ \\
\hline$C(426)$ & $0.261(1)$ & $0.2077(4)$ & $0.255(1)$ & $4.5(5)$ \\
\hline$C(511)$ & $-0.057(1)$ & $0.1307(3)$ & $0.108(1)$ & $2.7(4)$ \\
\hline
\end{tabular}




\begin{tabular}{|c|c|c|c|c|}
\hline$C(512)$ & $-0.113(1)$ & $0.1316(3)$ & $0.174(1)$ & $3.8(5)$ \\
\hline$C(513)$ & $-0.212(1)$ & $0.1338(4)$ & $0.157(1)$ & $4.8(5)$ \\
\hline$C(514)$ & $-0.255(2)$ & $0.1334(4)$ & $0.071(1)$ & $5.9(6)$ \\
\hline$C(515)$ & $-0.199(1)$ & $0.1323(4)$ & $-0.002(1)$ & $4.6(5)$ \\
\hline$C(516)$ & $-0.101(1)$ & $0.1311(3)$ & $0.018(1)$ & $3.8(5)$ \\
\hline$C(611)$ & $-0.052(1)$ & $0.0693(3)$ & $0.046(1)$ & $2.8(4)$ \\
\hline$C(612)$ & $-0.130(1)$ & $0.0701(3)$ & $0.096(1)$ & $4.0(5)$ \\
\hline$C(613)$ & $-0.228(2)$ & $0.0683(4)$ & $0.053(1)$ & $5.4(6)$ \\
\hline$C(614)$ & $-0.244(2)$ & $0.0666(4)$ & $-0.036(2)$ & $6.0(7)$ \\
\hline$C(615)$ & $-0.168(2)$ & $0.0658(4)$ & $-0.089(1)$ & $5.1(6)$ \\
\hline$C(616)$ & $-0.071(1)$ & $0.0672(3)$ & $-0.045(1)$ & $4.0(5)$ \\
\hline$C(621)$ & $0.142(1)$ & 0.0491(3) & $0.040(1)$ & $3.3(4)$ \\
\hline$C(622)$ & $0.106(1)$ & $0.0245(4)$ & $0.039(1)$ & $4.6(5)$ \\
\hline$C(623)$ & $0.162(2)$ & $0.0059(4)$ & $0.005(1)$ & $5.4(6)$ \\
\hline$C(624)$ & $0.244(2)$ & $0.0102(4)$ & $-0.026(1)$ & $5.2(6)$ \\
\hline$C(625)$ & $0.277(2)$ & $0.0351(4)$ & $-0.027(1)$ & $5.3(6)$ \\
\hline$C(626)$ & $0.224(1)$ & $0.0542(4)$ & $0.008(1)$ & $4.1(5)$ \\
\hline$C\left(711^{d}\right)$ & $0.554(2)$ & $0.1618(5)$ & $0.194(2)$ & $1.9(7)$ \\
\hline$C(712)^{d}$ & $0.534(4)$ & $0.1723(9)$ & $0.281(3)$ & $7(1)$ \\
\hline$C(713)^{d}$ & $0.581(5)$ & $0.177(1)$ & $0.131(5)$ & $10(2)$ \\
\hline$C(721)^{d}$ & $0.725(7)$ & $0.120(2)$ & $0.703(6)$ & $15(3)$ \\
\hline$C(722)^{d}$ & $0.802(4)$ & $0.1308(10)$ & $0.714(3)$ & $7(1)$ \\
\hline$C(723)^{d}$ & $0.633(4)$ & $0.1190(9)$ & $0.628(3)$ & $6(1)$ \\
\hline $\mathrm{H}(1)$ & 0.0864 & 0.1308 & 0.5459 & 2.8 \\
\hline $\mathrm{H}(2)$ & 0.0187 & 0.1195 & 0.4655 & 2.8 \\
\hline $\mathrm{H}(3)$ & 0.2742 & 0.0874 & 0.4523 & 2.6 \\
\hline $\mathrm{H}(4)$ & 0.2095 & 0.0901 & 0.5276 & 2.6 \\
\hline $\mathrm{H}(5)$ & 0.0721 & 0.1612 & 0.0232 & 2.9 \\
\hline $\mathrm{H}(6)$ & 0.1792 & 0.1563 & 0.0641 & 2.9 \\
\hline $\mathrm{H}(7)$ & 0.0883 & 0.1057 & 0.0021 & 3.1 \\
\hline $\mathrm{H}(8)$ & 0.1839 & 0.1029 & 0.0678 & 3.1 \\
\hline $\mathrm{H}(9)$ & -0.2526 & 0.2823 & 0.2480 & 11.0 \\
\hline $\mathrm{H}(10)$ & -0.1364 & 0.3134 & 0.2953 & 9.4 \\
\hline $\mathrm{H}(11)$ & 0.0199 & 0.3077 & 0.3478 & 9.5 \\
\hline $\mathrm{H}(12)$ & -0.3253 & -0.0165 & 0.2765 & 5.4 \\
\hline $\mathrm{H}(13)$ & -0.2589 & -0.0551 & 0.2568 & 5.5 \\
\hline $\mathrm{H}(14)$ & -0.0966 & -0.0580 & 0.2304 & 5.5 \\
\hline $\mathrm{H}(15)$ & -0.2815 & 0.2310 & 0.2943 & 7.8 \\
\hline $\mathrm{H}(16)$ & -0.2549 & 0.2308 & 0.1980 & 7.8 \\
\hline $\mathrm{H}(17)$ & -0.1941 & 0.2151 & 0.2723 & 7.8 \\
\hline $\mathrm{H}(18)$ & 0.1182 & 0.2516 & 0.4136 & 6.8 \\
\hline $\mathrm{H}(19)$ & 0.1376 & 0.2540 & 0.3154 & 6.8 \\
\hline $\mathrm{H}(20)$ & 0.1506 & 0.2772 & 0.3781 & 6.8 \\
\hline $\mathrm{H}(21)$ & -0.2036 & 0.0424 & 0.2699 & 4.8 \\
\hline $\mathrm{H}(22)$ & -0.3033 & 0.0320 & 0.2260 & 4.8 \\
\hline $\mathrm{H}(23)$ & -0.2752 & 0.0308 & 0.3288 & 4.8 \\
\hline $\mathrm{H}(24)$ & 0.0698 & -0.0380 & 0.2196 & 5.2 \\
\hline $\mathrm{H}(25)$ & 0.0556 & -0.0122 & 0.1718 & 5.2 \\
\hline $\mathrm{H}(26)$ & 0.0883 & -0.0133 & 0.2740 & 5.2 \\
\hline $\mathrm{H}(27)$ & 0.0478 & 0.1680 & 0.6339 & 5.0 \\
\hline $\mathrm{H}(28)$ & 0.1475 & 0.1925 & 0.7417 & 6.2 \\
\hline $\mathrm{H}(29)$ & 0.2487 & 0.2240 & 0.6977 & 5.8 \\
\hline $\mathrm{H}(30)$ & 0.2628 & 0.2294 & 0.5417 & 4.6 \\
\hline $\mathrm{H}(31)$ & 0.1666 & 0.2040 & 0.4327 & 4.9 \\
\hline $\mathrm{H}(32)$ & -0.0817 & 0.1962 & 0.5414 & 7.0 \\
\hline $\mathrm{H}(33)$ & -0.2537 & 0.1988 & 0.5461 & 8.4 \\
\hline $\mathrm{H}(34)$ & -0.3633 & 0.1707 & 0.4746 & 7.8 \\
\hline
\end{tabular}




\begin{tabular}{|c|c|c|c|c|}
\hline H(35) & -0.2988 & 0.1355 & 0.3944 & 7.2 \\
\hline $\mathrm{H}(36)$ & -0.1306 & 0.1341 & 0.3782 & 5.1 \\
\hline $\mathrm{H}(37)$ & 0.2669 & 0.1292 & 0.6092 & 4.5 \\
\hline $\mathrm{H}(38)$ & 0.3915 & 0.1559 & 0.6795 & 6.3 \\
\hline H(39) & 0.4803 & 0.1817 & 0.5916 & 6.3 \\
\hline $\mathrm{H}(40)$ & 0.4351 & 0.1835 & 0.4330 & 5.2 \\
\hline $\mathrm{H}(41)$ & 0.3040 & 0.1574 & 0.3692 & 3.2 \\
\hline $\mathrm{H}(42)$ & 0.0741 & 0.0642 & 0.5888 & 3.4 \\
\hline $\mathrm{H}(43)$ & -0.0669 & 0.0710 & 0.6527 & 4.9 \\
\hline $\mathrm{H}(44)$ & -0.2076 & 0.0806 & 0.5679 & 5.4 \\
\hline $\mathrm{H}(45)$ & -0.2174 & 0.0854 & 0.4130 & 5.1 \\
\hline $\mathrm{H}(46)$ & -0.0756 & 0.0769 & 0.3433 & 4.0 \\
\hline $\mathrm{H}(47)$ & 0.0477 & 0.0186 & 0.4421 & 3.4 \\
\hline $\mathrm{H}(48)$ & 0.1170 & -0.0217 & 0.4881 & 4.7 \\
\hline H(49) & 0.2826 & -0.0254 & 0.5214 & 5.3 \\
\hline $\mathrm{H}(50)$ & 0.3933 & 0.0083 & 0.5080 & 5.9 \\
\hline $\mathrm{H}(51)$ & 0.3207 & 0.0493 & 0.4732 & 4.4 \\
\hline $\mathrm{H}(52)$ & -0.0965 & 0.1804 & 0.0763 & 4.2 \\
\hline H(53) & -0.2071 & 0.2118 & 0.0120 & 5.7 \\
\hline $\mathrm{H}(54)$ & -0.1622 & 0.2541 & 0.0068 & 4.9 \\
\hline $\mathrm{H}(55)$ & -0.0085 & 0.2668 & 0.0667 & 5.9 \\
\hline $\mathrm{H}(56)$ & 0.1074 & 0.2365 & 0.1300 & 4.3 \\
\hline $\mathrm{H}(57)$ & 0.2378 & 0.1975 & 0.0367 & 5.1 \\
\hline H(58) & 0.3799 & 0.2252 & 0.0530 & 5.7 \\
\hline H(59) & 0.4427 & 0.2395 & 0.1931 & 6.2 \\
\hline $\mathrm{H}(60)$ & 0.3790 & 0.2290 & 0.3208 & 5.9 \\
\hline $\mathrm{H}(61)$ & 0.2300 & 0.2045 & 0.3079 & 4.9 \\
\hline $\mathrm{H}(62)$ & -0.0814 & 0.1300 & 0.2385 & 4.1 \\
\hline $\mathrm{H}(63)$ & -0.2496 & 0.1353 & 0.2039 & 4.8 \\
\hline $\mathrm{H}(64)$ & -0.3261 & 0.1360 & 0.0582 & 6.3 \\
\hline $\mathrm{H}(65)$ & -0.2305 & 0.1322 & -0.0626 & 5.0 \\
\hline $\mathrm{H}(66)$ & -0.0604 & 0.1301 & -0.0289 & 3.7 \\
\hline $\mathrm{H}(67)$ & -0.1163 & 0.0723 & 0.1598 & 4.5 \\
\hline $\mathrm{H}(68)$ & -0.2797 & 0.0694 & 0.0898 & 5.4 \\
\hline H(69) & -0.3102 & 0.0679 & -0.0611 & 6.6 \\
\hline $\mathrm{H}(70)$ & -0.1804 & 0.0635 & -0.1522 & 6.1 \\
\hline $\mathrm{H}(71)$ & -0.0135 & 0.0658 & -0.0784 & 4.2 \\
\hline $\mathrm{H}(72)$ & 0.0473 & 0.0200 & 0.0646 & 5.0 \\
\hline $\mathrm{H}(73)$ & 0.1392 & -0.0106 & 0.0060 & 5.3 \\
\hline $\mathrm{H}(74)$ & 0.2805 & -0.0027 & -0.0503 & 5.4 \\
\hline $\mathrm{H}(75)$ & 0.3364 & 0.0378 & -0.0504 & 6.4 \\
\hline $\mathrm{H}(76)$ & 0.2454 & 0.0711 & 0.0072 & 4.1 \\
\hline
\end{tabular}

a Estimated standard deviations are given in parentheses. ${ }^{b}$ The $P t, P, F, O, N$, and $C(1)-C(48)$ were assigned with anisotropic thermal parameters given as the isotropic equivalent displacement parameter defined as $B_{\text {eq }}=\left(8 \pi^{2} / 3\right)\left(U_{11}\left(a a^{*}\right)^{2}+U_{22}\left(b b^{*}\right)^{2}+U_{33}\left(c c^{*}\right)^{2}+2 U_{12} a a^{*} b b^{*} \cos \gamma+\right.$ $\left.2 U_{13} a a^{*} c c^{*} \cos \beta+2 U_{23} b b^{*} c c^{*} \cos \alpha\right)$. The phenyl groups and the lattice acetones were refined isotropically. ${ }^{\mathrm{C}}$ All C-H hydrogen atoms except those of acetones were calculated at ideal positions with C-H $=0.95 \AA$ and were not refined. d Disordered with 0.5 occupancy.

Table S9-3. Final Anisotropic Thermal Parameters for [ $\mathrm{Pt}_{3}(\mu-\mathrm{dpmp})_{2}(\mu-$ $\left.\left.\mathrm{C}_{12} \mathrm{~N}_{8}\right)(\mathrm{XylNC})_{2}\right]\left(\mathrm{PF}_{6}\right)_{2} \cdot 2\left(\mathrm{CH}_{3}\right)_{2} \mathrm{CO}\left(\mathbf{1 1 a} \cdot\left(\mathrm{CH}_{3}\right)_{2} \mathrm{CO}\right)^{\mathrm{a}, \mathrm{b}}$

\begin{tabular}{lcccccc}
\hline atom & $U_{11}$ & $U_{22}$ & $U_{33}$ & $U_{12}$ & $U_{13}$ & $U_{23}$ \\
\hline $\operatorname{Pt}(1)$ & $0.0434(4)$ & $0.0206(4)$ & $0.0413(4)$ & $0.0012(3)$ & $0.0098(3)$ & $0.0018(3)$ \\
$\operatorname{Pt}(2)$ & $0.0391(4)$ & $0.0217(4)$ & $0.0373(4)$ & $0.0017(3)$ & $0.0062(3)$ & $0.0012(3)$ \\
$\operatorname{Pt}(3)$ & $0.0430(4)$ & $0.0231(4)$ & $0.0372(4)$ & $0.0016(3)$ & $0.0035(3)$ & $0.0002(3)$
\end{tabular}




\begin{tabular}{|c|c|c|c|c|c|c|}
\hline $\mathrm{P}(1)$ & $0.056(3)$ & $0.021(2)$ & $0.049(3)$ & $0.002(2)$ & $0.015(2)$ & $0.001(2)$ \\
\hline $\mathrm{P}(2)$ & $0.041(3)$ & $0.025(2)$ & $0.040(2)$ & $0.000(2)$ & $0.005(2)$ & $-0.002(2)$ \\
\hline $\mathrm{P}(3)$ & $0.042(3)$ & $0.025(2)$ & $0.039(2)$ & $0.003(2)$ & $0.000(2)$ & $0.004(2)$ \\
\hline $\mathrm{P}(4)$ & $0.047(3)$ & $0.022(2)$ & $0.042(3)$ & $0.004(2)$ & $0.011(2)$ & $0.004(2)$ \\
\hline $\mathrm{P}(5)$ & $0.037(3)$ & $0.026(2)$ & $0.042(2)$ & $0.001(2)$ & $0.007(2)$ & $-0.002(2)$ \\
\hline$P(6)$ & $0.051(3)$ & $0.025(2)$ & $0.036(2)$ & $0.005(2)$ & $0.005(2)$ & $0.000(2)$ \\
\hline $\mathrm{P}(11)$ & $0.057(4)$ & $0.059(4)$ & $0.149(7)$ & $0.003(3)$ & $0.008(4)$ & $-0.034(4)$ \\
\hline $\mathrm{P}(12)$ & $0.222(10)$ & $0.047(4)$ & $0.075(5)$ & $0.008(5)$ & $0.071(6)$ & $0.018(3)$ \\
\hline $\mathrm{F}(11)$ & $0.27(3)$ & $0.19(3)$ & $0.28(3)$ & $0.00(2)$ & $-0.17(3)$ & $-0.01(2)$ \\
\hline $\mathrm{F}(12)$ & $0.12(1)$ & $0.13(2)$ & $0.44(4)$ & $-0.03(1)$ & $0.11(2)$ & $-0.18(2)$ \\
\hline $\mathrm{F}(13)$ & $0.12(1)$ & $0.14(2)$ & $0.35(3)$ & $-0.08(1)$ & $0.13(2)$ & $-0.12(2)$ \\
\hline $\mathrm{F}(14)$ & $0.19(2)$ & $0.09(1)$ & $0.52(5)$ & $-0.04(1)$ & $0.26(3)$ & $-0.07(2)$ \\
\hline $\mathrm{F}(15)$ & $0.45(6)$ & $0.36(5)$ & $0.15(2)$ & $0.23(4)$ & $-0.06(3)$ & $0.00(3)$ \\
\hline $\mathrm{F}(16)$ & $0.08(1)$ & $0.12(1)$ & $0.29(3)$ & $-0.009(10)$ & $0.05(1)$ & $-0.11(2)$ \\
\hline $\mathrm{F}(21)$ & $0.22(2)$ & $0.059(9)$ & $0.09(1)$ & $-0.03(1)$ & $0.02(1)$ & $0.010(8)$ \\
\hline $\mathrm{F}(22)$ & $0.21(2)$ & $0.12(1)$ & $0.13(1)$ & $-0.02(1)$ & $0.05(1)$ & $0.05(1)$ \\
\hline $\mathrm{F}(23)$ & $0.32(3)$ & $0.10(1)$ & $0.12(1)$ & $-0.01(2)$ & $0.09(2)$ & $-0.04(1)$ \\
\hline $\mathrm{F}(24)$ & $0.19(2)$ & $0.18(2)$ & $0.12(1)$ & $0.02(2)$ & $0.04(1)$ & $0.08(1)$ \\
\hline $\mathrm{F}(25)$ & $0.25(2)$ & $0.09(1)$ & $0.19(2)$ & $0.03(1)$ & $0.14(2)$ & $0.08(1)$ \\
\hline $\mathrm{F}(26)$ & $0.23(2)$ & $0.08(1)$ & $0.11(1)$ & $-0.04(1)$ & $0.08(1)$ & $-0.011(9)$ \\
\hline $\mathrm{N}(1)$ & $0.10(1)$ & $0.023(8)$ & $0.044(9)$ & $0.015(9)$ & $0.026(9)$ & $0.013(7)$ \\
\hline $\mathrm{N}(2)$ & $0.057(10)$ & $0.041(9)$ & $0.030(8)$ & $0.017(8)$ & $0.005(7)$ & $0.002(7)$ \\
\hline $\mathrm{N}(11)$ & $0.043(10)$ & $0.042(9)$ & $0.041(9)$ & $-0.014(7)$ & $0.004(7)$ & $-0.001(7)$ \\
\hline $\mathrm{N}(21)$ & $0.08(1)$ & $0.025(9)$ & $0.08(1)$ & $0.010(9)$ & $0.003(10)$ & $-0.007(8)$ \\
\hline $\mathrm{N}(22)$ & $0.10(2)$ & $0.09(2)$ & $0.05(1)$ & $0.05(1)$ & $0.00(1)$ & $-0.01(1)$ \\
\hline $\mathrm{N}(23)$ & $0.14(2)$ & $0.15(2)$ & $0.08(1)$ & $0.11(2)$ & $0.00(1)$ & $-0.07(1)$ \\
\hline $\mathrm{N}(24)$ & $0.06(1)$ & $0.07(1)$ & $0.12(2)$ & $-0.02(1)$ & $0.01(1)$ & $0.01(1)$ \\
\hline $\mathrm{N}(25)$ & $0.12(2)$ & $0.17(3)$ & $0.06(1)$ & $-0.04(2)$ & $0.01(1)$ & $0.00(1)$ \\
\hline $\mathrm{N}(26)$ & $0.06(1)$ & $0.06(1)$ & $0.07(1)$ & $0.012(9)$ & $0.006(10)$ & $-0.025(10)$ \\
\hline $\mathrm{N}(27)$ & $0.05(1)$ & $0.05(1)$ & $0.13(2)$ & $0.008(9)$ & $0.02(1)$ & $0.02(1)$ \\
\hline$C(1)$ & $0.07(1)$ & $0.05(1)$ & $0.04(1)$ & $0.00(1)$ & $0.018(10)$ & $0.021(9)$ \\
\hline$C(2)$ & $0.04(1)$ & $0.001(7)$ & $0.08(1)$ & $0.005(8)$ & $-0.007(9)$ & $-0.006(8)$ \\
\hline$C(3)$ & $0.06(1)$ & $0.012(8)$ & $0.038(9)$ & $0.008(7)$ & $-0.003(8)$ & $-0.012(7)$ \\
\hline$C(4)$ & $0.05(1)$ & $0.037(10)$ & $0.036(10)$ & $-0.002(8)$ & $-0.007(8)$ & $0.000(8)$ \\
\hline$C(5)$ & $0.041(10)$ & $0.021(8)$ & $0.047(10)$ & $-0.001(7)$ & $0.015(8)$ & $-0.003(7)$ \\
\hline$C(6)$ & $0.04(1)$ & $0.026(9)$ & $0.041(10)$ & $0.002(8)$ & $0.003(8)$ & $0.013(7)$ \\
\hline$C(11)$ & $0.03(1)$ & $0.05(1)$ & $0.05(1)$ & $0.007(9)$ & $0.002(8)$ & $0.013(9)$ \\
\hline$C(12)$ & $0.05(1)$ & $0.04(1)$ & $0.06(1)$ & $0.004(9)$ & $0.023(9)$ & $-0.004(8)$ \\
\hline$C(13)$ & $0.06(1)$ & $0.06(1)$ & $0.07(1)$ & $0.02(1)$ & $0.01(1)$ & $0.00(1)$ \\
\hline$C(14)$ & $0.07(1)$ & $0.07(1)$ & $0.06(1)$ & $0.01(1)$ & $0.00(1)$ & $-0.02(1)$ \\
\hline$C(15)$ & $0.031(9)$ & $0.032(9)$ & $0.05(1)$ & $0.003(8)$ & $0.008(8)$ & $0.002(8)$ \\
\hline$C(21)$ & $0.04(1)$ & $0.04(1)$ & $0.05(1)$ & $0.018(10)$ & $-0.014(9)$ & $-0.001(9)$ \\
\hline$C(22)$ & $0.06(1)$ & $0.05(1)$ & $0.07(2)$ & $0.02(1)$ & $0.02(1)$ & $-0.02(1)$ \\
\hline$C(23)$ & $0.07(2)$ & $0.08(2)$ & $0.10(2)$ & $0.02(1)$ & $-0.01(1)$ & $-0.01(1)$ \\
\hline$C(24)$ & $0.04(1)$ & $0.06(1)$ & $0.08(1)$ & $0.00(1)$ & $0.02(1)$ & $0.00(1)$ \\
\hline$C(25)$ & $0.04(1)$ & $0.12(2)$ & $0.07(2)$ & $0.00(1)$ & $0.02(1)$ & $0.00(1)$ \\
\hline$C(26)$ & $0.06(1)$ & $0.07(1)$ & $0.07(2)$ & $-0.02(1)$ & $0.01(1)$ & $-0.03(1)$ \\
\hline$C(27)$ & $0.04(1)$ & $0.04(1)$ & $0.11(2)$ & $0.00(1)$ & $0.00(1)$ & $0.03(1)$ \\
\hline$C(31)$ & $0.14(2)$ & $0.02(1)$ & $0.05(1)$ & $0.01(1)$ & $0.04(1)$ & $0.004(9)$ \\
\hline$C(32)$ & $0.11(2)$ & $0.06(2)$ & $0.10(2)$ & $0.05(2)$ & $0.00(2)$ & $0.02(1)$ \\
\hline$C(33)$ & $0.15(3)$ & $0.23(5)$ & $0.10(2)$ & $0.14(3)$ & $0.02(2)$ & $0.03(3)$ \\
\hline$C(34)$ & $0.21(5)$ & $0.05(2)$ & $0.14(3)$ & $0.01(3)$ & $0.06(3)$ & $0.02(2)$ \\
\hline$C(35)$ & $0.16(3)$ & $0.05(2)$ & $0.14(3)$ & $0.05(2)$ & $0.03(2)$ & $0.01(2)$ \\
\hline$C(36)$ & $0.11(2)$ & $0.07(2)$ & $0.08(2)$ & $0.03(2)$ & $0.04(2)$ & $0.01(1)$ \\
\hline C(37) & $0.10(2)$ & $0.09(2)$ & $0.09(2)$ & $0.00(2)$ & $0.00(2)$ & $-0.01(2)$ \\
\hline$C(38)$ & $0.15(3)$ & $0.04(1)$ & $0.09(2)$ & $-0.02(2)$ & $0.04(2)$ & $0.00(1)$ \\
\hline$C(41)$ & $0.07(1)$ & $0.03(1)$ & $0.04(1)$ & $-0.030(10)$ & $-0.010(9)$ & $0.001(8)$ \\
\hline$C(42)$ & $0.05(1)$ & $0.04(1)$ & $0.06(1)$ & $-0.011(9)$ & $0.008(9)$ & $-0.008(9)$ \\
\hline
\end{tabular}




\begin{tabular}{llllrrc}
$\mathrm{C}(43)$ & $0.06(1)$ & $0.07(2)$ & $0.05(1)$ & $-0.02(1)$ & $0.01(1)$ & $0.02(1)$ \\
$\mathrm{C}(44)$ & $0.07(2)$ & $0.03(1)$ & $0.09(2)$ & $-0.03(1)$ & $-0.01(1)$ & $0.00(1)$ \\
$\mathrm{C}(45)$ & $0.15(2)$ & $0.02(1)$ & $0.03(1)$ & $0.00(1)$ & $0.00(1)$ & $-0.013(8)$ \\
$\mathrm{C}(46)$ & $0.08(2)$ & $0.04(1)$ & $0.05(1)$ & $0.01(1)$ & $0.00(1)$ & $-0.007(9)$ \\
$\mathrm{C}(47)$ & $0.05(1)$ & $0.05(1)$ & $0.10(2)$ & $-0.005(10)$ & $0.03(1)$ & $0.02(1)$ \\
$\mathrm{C}(48)$ & $0.10(2)$ & $0.027(10)$ & $0.06(1)$ & $0.00(1)$ & $0.01(1)$ & $-0.007(9)$ \\
\hline
\end{tabular}

a Estmated standard deviations are given in parentheses.

$\mathrm{b}$ Parameters have the form $\exp \left(-2 \pi^{2}\left(U_{11} h^{2} a^{* 2}+\cdots+2 U_{12} h k a^{*} b^{*} \cos \gamma+\cdots\right)\right)$.

Table S9-4. Bond Lengths for Non-hydrogen Atoms of [Pt3( $\mu$-dpmp) $2(\mu-$

$\left.\left.\mathrm{C}_{12} \mathrm{~N}_{8}\right)(\mathrm{Xy1NC})_{2}\right]\left(\mathrm{PF}_{6}\right)_{2} \cdot 2\left(\mathrm{CH}_{3}\right)_{2} \mathrm{CO}\left(\mathbf{1 1 a} \cdot\left(\mathrm{CH}_{3}\right)_{2} \mathrm{CO}\right)^{\mathrm{a}}$

\begin{tabular}{|c|c|c|c|c|c|}
\hline atom & atom & distance & atom & atom & distance \\
\hline$\overline{\operatorname{Pt}(1)}$ & $\operatorname{Pt}(2)$ & $2.6446(8)$ & $P(6)$ & $C(6)$ & $1.81(2)$ \\
\hline $\operatorname{Pt}(1)$ & $\mathrm{P}(1)$ & $2.312(5)$ & $P(6)$ & $C(611)$ & $1.83(2)$ \\
\hline $\operatorname{Pt}(1)$ & $\mathrm{P}(4)$ & $2.328(5)$ & $P(6)$ & $C(621)$ & $1.84(2)$ \\
\hline $\operatorname{Pt}(1)$ & $C(1)$ & $2.01(2)$ & $\mathrm{P}(11)$ & $\mathrm{F}(11)$ & $1.44(3)$ \\
\hline $\operatorname{Pt}(2)$ & $P(2)$ & $2.263(4)$ & $P(11)$ & $\mathrm{F}(12)$ & $1.50(2)$ \\
\hline $\operatorname{Pt}(2)$ & $P(5)$ & $2.277(4)$ & $\mathrm{P}(11)$ & $\mathrm{F}(13)$ & $1.55(2)$ \\
\hline $\operatorname{Pt}(2)$ & $\mathrm{N}(11)$ & $2.18(1)$ & $\mathrm{P}(11)$ & $\mathrm{F}(14)$ & $1.54(3)$ \\
\hline $\operatorname{Pt}(3)$ & $P(3)$ & $2.341(4)$ & $\mathrm{P}(11)$ & $\mathrm{F}(15)$ & $1.46(3)$ \\
\hline $\operatorname{Pt}(3)$ & $P(6)$ & $2.349(4)$ & $\mathrm{P}(11)$ & $\mathrm{F}(16)$ & $1.57(2)$ \\
\hline $\operatorname{Pt}(3)$ & $\mathrm{N}(11)$ & $2.05(1)$ & $\mathrm{P}(12)$ & $\mathrm{F}(21)$ & $1.57(2)$ \\
\hline $\operatorname{Pt}(3)$ & $C(2)$ & $1.90(2)$ & $\mathrm{P}(12)$ & $\mathrm{F}(22)$ & $1.63(2)$ \\
\hline$P(1)$ & $C(3)$ & $1.85(1)$ & $\mathrm{P}(12)$ & $\mathrm{F}(23)$ & $1.56(2)$ \\
\hline $\mathrm{P}(1)$ & $C(111)$ & $1.82(2)$ & $\mathrm{P}(12)$ & $\mathrm{F}(24)$ & $1.57(2)$ \\
\hline $\mathrm{P}(1)$ & $C(121)$ & $1.81(2)$ & $\mathrm{P}(12)$ & $\mathrm{F}(25)$ & $1.57(2)$ \\
\hline$P(2)$ & $C(3)$ & $1.84(2)$ & $P(12)$ & $\mathrm{F}(26)$ & $1.57(2)$ \\
\hline $\mathrm{P}(2)$ & $C(4)$ & $1.84(2)$ & $\mathrm{O}(701)$ & $\mathrm{C}(711)$ & $1.05(4)$ \\
\hline $\mathrm{P}(2)$ & $C(211)$ & $1.82(2)$ & $\mathrm{O}(702)$ & $\mathrm{C}(721)$ & $1.5(1)$ \\
\hline $\mathrm{P}(3)$ & $C(4)$ & $1.84(2)$ & $\mathrm{N}(1)$ & $C(1)$ & $1.11(3)$ \\
\hline$P(3)$ & $C(311)$ & $1.84(2)$ & $\mathrm{N}(1)$ & $C(31)$ & $1.45(2)$ \\
\hline$P(3)$ & $C(321)$ & $1.82(2)$ & $\mathrm{N}(2)$ & $C(2)$ & $1.16(2)$ \\
\hline$P(4)$ & $C(5)$ & $1.85(2)$ & $\mathrm{N}(2)$ & $C(41)$ & $1.48(2)$ \\
\hline $\mathrm{P}(4)$ & $C(411)$ & $1.85(2)$ & $\mathrm{N}(11)$ & $C(11)$ & $1.26(2)$ \\
\hline $\mathrm{P}(4)$ & $C(421)$ & $1.79(2)$ & $\mathrm{N}(21)$ & $C(21)$ & $1.10(2)$ \\
\hline$P(5)$ & $C(5)$ & $1.84(2)$ & $\mathrm{N}(22)$ & $C(22)$ & $1.14(3)$ \\
\hline$P(5)$ & $C(6)$ & $1.84(2)$ & $\mathrm{N}(23)$ & $C(23)$ & $1.13(4)$ \\
\hline$P(5)$ & $C(511)$ & $1.84(2)$ & $\mathrm{N}(24)$ & $C(24)$ & $1.09(3)$ \\
\hline $\mathrm{N}(25)$ & $C(25)$ & 1.11(3) & $C(42)$ & $C(47)$ & $1.46(3)$ \\
\hline $\mathrm{N}(26)$ & $C(26)$ & 1.11(3) & $C(43)$ & $C(44)$ & $1.30(3)$ \\
\hline $\mathrm{N}(27)$ & $C(27)$ & $1.05(3)$ & $C(44)$ & $C(45)$ & $1.37(4)$ \\
\hline$C(11)$ & $C(12)$ & $1.46(2)$ & $C(45)$ & $C(46)$ & $1.39(3)$ \\
\hline$C(11)$ & $C(15)$ & $1.54(2)$ & $C(46)$ & $C(48)$ & $1.51(3)$ \\
\hline$C(12)$ & $C(13)$ & $1.54(3)$ & $C(111)$ & $C(112)$ & $1.39(3)$ \\
\hline$C(12)$ & $C(21)$ & $1.40(3)$ & $C(111)$ & $C(116)$ & $1.37(3)$ \\
\hline$C(13)$ & $C(14)$ & $1.60(3)$ & $C(112)$ & $C(113)$ & $1.42(3)$ \\
\hline$C(13)$ & $C(22)$ & $1.52(3)$ & $C(113)$ & $C(114)$ & $1.35(3)$ \\
\hline$C(13)$ & $C(23)$ & $1.59(3)$ & $C(114)$ & $C(115)$ & $1.42(3)$ \\
\hline$C(14)$ & $C(15)$ & $1.57(3)$ & $C(115)$ & $C(116)$ & $1.39(3)$ \\
\hline$C(14)$ & $C(24)$ & $1.52(3)$ & $C(121)$ & $C(122)$ & $1.41(3)$ \\
\hline$C(14)$ & $C(25)$ & $1.51(3)$ & $C(121)$ & $C(126)$ & $1.36(3)$ \\
\hline$C(15)$ & $C(26)$ & $1.52(3)$ & $C(122)$ & $C(123)$ & $1.47(4)$ \\
\hline$C(15)$ & $C(27)$ & $1.49(3)$ & $C(123)$ & $C(124)$ & $1.30(3)$ \\
\hline$C(31)$ & $C(32)$ & $1.41(4)$ & $C(124)$ & $C(125)$ & $1.44(3)$ \\
\hline$C(31)$ & $C(36)$ & $1.37(3)$ & $C(125)$ & $C(126)$ & $1.40(3)$ \\
\hline
\end{tabular}




\begin{tabular}{|c|c|c|c|c|c|}
\hline$C(32)$ & $C(33)$ & $1.35(5)$ & $C(211)$ & $C(212)$ & $1.39(2)$ \\
\hline$C(32)$ & $C(37)$ & $1.46(4)$ & $C(211)$ & $C(216)$ & $1.35(2)$ \\
\hline$C(33)$ & $C(34)$ & $1.58(6)$ & $C(212)$ & $C(213)$ & $1.38(3)$ \\
\hline$C(34)$ & $C(35)$ & $1.17(6)$ & $C(213)$ & $C(214)$ & $1.36(3)$ \\
\hline$C(35)$ & $C(36)$ & $1.36(4)$ & $C(214)$ & $C(215)$ & $1.38(3)$ \\
\hline$C(36)$ & $C(38)$ & $1.48(4)$ & $C(215)$ & $C(216)$ & $1.42(3)$ \\
\hline$C(41)$ & $C(42)$ & $1.36(3)$ & $C(311)$ & $C(312)$ & $1.41(2)$ \\
\hline$C(41)$ & $C(46)$ & $1.42(3)$ & $C(311)$ & $C(316)$ & $1.38(2)$ \\
\hline$C(42)$ & $C(43)$ & $1.39(3)$ & $C(312)$ & $C(313)$ & $1.34(3)$ \\
\hline$C(313)$ & $C(314)$ & $1.32(3)$ & $C(515)$ & $C(516)$ & $1.35(3)$ \\
\hline$C(314)$ & $C(315)$ & $1.39(3)$ & $C(611)$ & $C(612)$ & $1.41(3)$ \\
\hline$C(315)$ & $C(316)$ & $1.41(3)$ & $C(611)$ & $C(616)$ & $1.36(2)$ \\
\hline$C(321)$ & $C(322)$ & $1.38(2)$ & $C(612)$ & $C(613)$ & $1.42(3)$ \\
\hline$C(321)$ & $C(326)$ & $1.36(3)$ & $C(613)$ & $C(614)$ & $1.33(3)$ \\
\hline$C(322)$ & $C(323)$ & $1.43(3)$ & $C(614)$ & $C(615)$ & $1.42(3)$ \\
\hline$C(323)$ & $C(324)$ & $1.34(3)$ & $C(615)$ & $C(616)$ & $1.42(3)$ \\
\hline$C(324)$ & $C(325)$ & $1.38(3)$ & $C(621)$ & $C(622)$ & $1.40(3)$ \\
\hline$C(325)$ & $C(326)$ & $1.44(3)$ & $C(621)$ & $C(626)$ & $1.32(3)$ \\
\hline$C(411)$ & $C(412)$ & $1.38(2)$ & $C(622)$ & $C(623)$ & $1.40(3)$ \\
\hline$C(411)$ & $C(416)$ & $1.40(2)$ & $C(623)$ & $C(624)$ & $1.31(3)$ \\
\hline$C(412)$ & $C(413)$ & $1.40(3)$ & $C(624)$ & $C(625)$ & $1.40(3)$ \\
\hline$C(413)$ & $C(414)$ & $1.39(3)$ & $C(625)$ & $C(626)$ & $1.40(3)$ \\
\hline$C(414)$ & $C(415)$ & $1.37(3)$ & $\mathrm{C}(711)$ & $C(712)$ & $1.49(6)$ \\
\hline$C(415)$ & $C(416)$ & $1.37(3)$ & $\mathrm{C}(711)$ & $C(713)$ & $1.35(8)$ \\
\hline$C(421)$ & $C(422)$ & $1.42(3)$ & $C(721)$ & $C(722)$ & $1.2(1)$ \\
\hline$C(421)$ & $C(426)$ & $1.35(2)$ & $C(721)$ & $C(723)$ & $1.59(9)$ \\
\hline$C(422)$ & $C(423)$ & $1.45(3)$ & & & \\
\hline$C(423)$ & $C(424)$ & $1.36(3)$ & & & \\
\hline$C(424)$ & $C(425)$ & $1.33(3)$ & & & \\
\hline$C(425)$ & $C(426)$ & $1.45(3)$ & & & \\
\hline$C(511)$ & $C(512)$ & $1.35(3)$ & & & \\
\hline$C(511)$ & $C(516)$ & $1.40(2)$ & & & \\
\hline$C(512)$ & $C(513)$ & $1.36(3)$ & & & \\
\hline$C(513)$ & $C(514)$ & $1.35(3)$ & & & \\
\hline$C(514)$ & $C(515)$ & $1.43(3)$ & & & \\
\hline
\end{tabular}

a Estimated standard deviations are given in parentheses.

Table S9-5. Bond Angles for Non-hydrogen Atoms of $\left[\mathrm{Pt}_{3}(\mu-\mathrm{dpmp})_{2}\left(\mu-\mathrm{C}_{12} \mathrm{~N}_{8}\right)(\mathrm{XylNC})_{2}\right]-$ $\left(\mathrm{PF}_{6}\right)_{2} \cdot 2\left(\mathrm{CH}_{3}\right)_{2} \mathrm{CO}\left(\mathbf{1 1 a} \cdot\left(\mathrm{CH}_{3}\right)_{2} \mathrm{CO}\right)^{\mathrm{a}}$

\begin{tabular}{lllrllll}
\hline atom & atom & atom & \multicolumn{1}{c}{ angle } & atom & atom & atom & angle \\
\hline $\operatorname{Pt}(2)$ & $\mathrm{Pt}(1)$ & $\mathrm{P}(1)$ & $93.9(1)$ & $\mathrm{Pt}(2)$ & $\mathrm{P}(2)$ & $\mathrm{C}(211)$ & $118.2(5)$ \\
$\mathrm{Pt}(2)$ & $\mathrm{Pt}(1)$ & $\mathrm{P}(4)$ & $88.9(1)$ & $\mathrm{C}(3)$ & $\mathrm{P}(2)$ & $\mathrm{C}(4)$ & $103.4(7)$ \\
$\mathrm{Pt}(2)$ & $\mathrm{Pt}(1)$ & $\mathrm{C}(1)$ & $177.8(6)$ & $\mathrm{C}(3)$ & $\mathrm{P}(2)$ & $\mathrm{C}(211)$ & $104.8(7)$ \\
$\mathrm{P}(1)$ & $\mathrm{Pt}(1)$ & $\mathrm{P}(4)$ & $173.4(2)$ & $\mathrm{C}(4)$ & $\mathrm{P}(2)$ & $\mathrm{C}(211)$ & $105.5(7)$ \\
$\mathrm{P}(1)$ & $\mathrm{Pt}(1)$ & $\mathrm{C}(1)$ & $87.4(5)$ & $\mathrm{Pt}(3)$ & $\mathrm{P}(3)$ & $\mathrm{C}(4)$ & $115.8(6)$ \\
$\mathrm{P}(4)$ & $\mathrm{Pt}(1)$ & $\mathrm{C}(1)$ & $89.6(5)$ & $\mathrm{Pt}(3)$ & $\mathrm{P}(3)$ & $\mathrm{C}(311)$ & $112.2(5)$ \\
$\mathrm{Pt}(1)$ & $\mathrm{Pt}(2)$ & $\mathrm{P}(2)$ & $89.5(1)$ & $\mathrm{Pt}(3)$ & $\mathrm{P}(3)$ & $\mathrm{C}(321)$ & $113.6(5)$ \\
$\mathrm{Pt}(1)$ & $\mathrm{Pt}(2)$ & $\mathrm{P}(5)$ & $87.3(1)$ & $\mathrm{C}(4)$ & $\mathrm{P}(3)$ & $\mathrm{C}(311)$ & $104.3(8)$ \\
$\mathrm{Pt}(1)$ & $\mathrm{Pt}(2)$ & $\mathrm{N}(11)$ & $175.1(4)$ & $\mathrm{C}(4)$ & $\mathrm{P}(3)$ & $\mathrm{C}(321)$ & $104.1(7)$ \\
$\mathrm{P}(2)$ & $\mathrm{Pt}(2)$ & $\mathrm{P}(5)$ & $169.6(2)$ & $\mathrm{C}(311)$ & $\mathrm{P}(3)$ & $\mathrm{C}(321)$ & $105.8(8)$ \\
$\mathrm{P}(2)$ & $\mathrm{Pt}(2)$ & $\mathrm{N}(11)$ & $92.2(4)$ & $\mathrm{Pt}(1)$ & $\mathrm{P}(4)$ & $\mathrm{C}(5)$ & $116.9(5)$ \\
$\mathrm{P}(5)$ & $\mathrm{Pt}(2)$ & $\mathrm{N}(11)$ & $91.8(4)$ & $\mathrm{Pt}(1)$ & $\mathrm{P}(4)$ & $\mathrm{C}(411)$ & $112.0(6)$ \\
$\mathrm{P}(3)$ & $\mathrm{Pt}(3)$ & $\mathrm{P}(6)$ & $174.9(1)$ & $\mathrm{Pt}(1)$ & $\mathrm{P}(4)$ & $\mathrm{C}(421)$ & $110.6(5)$ \\
$\mathrm{P}(3)$ & $\mathrm{Pt}(3)$ & $\mathrm{N}(11)$ & $89.3(4)$ & $\mathrm{C}(5)$ & $\mathrm{P}(4)$ & $\mathrm{C}(411)$ & $105.3(7)$ \\
$\mathrm{P}(3)$ & $\mathrm{Pt}(3)$ & $\mathrm{C}(2)$ & $91.8(6)$ & $\mathrm{C}(5)$ & $\mathrm{P}(4)$ & $\mathrm{C}(421)$ & $106.6(8)$
\end{tabular}




\begin{tabular}{|c|c|c|c|c|c|c|c|}
\hline $\mathrm{P}(6)$ & $\operatorname{Pt}(3)$ & $\mathrm{N}(11)$ & $87.3(4)$ & $C(411)$ & $\mathrm{P}(4)$ & $C(421)$ & $104.5(7)$ \\
\hline$P(6)$ & $\mathrm{Pt}(3)$ & $C(2)$ & $92.0(6)$ & $\operatorname{Pt}(2)$ & $P(5)$ & $C(5)$ & $111.5(5)$ \\
\hline $\mathrm{N}(11)$ & $\mathrm{Pt}(3)$ & $C(2)$ & $173.0(6)$ & $\mathrm{Pt}(2)$ & $P(5)$ & $C(6)$ & $112.6(5)$ \\
\hline $\operatorname{Pt}(1)$ & $\mathrm{P}(1)$ & $C(3)$ & $114.3(6)$ & $\operatorname{Pt}(2)$ & $P(5)$ & $C(511)$ & $116.7(5)$ \\
\hline $\operatorname{Pt}(1)$ & $\mathrm{P}(1)$ & $C(111)$ & $112.2(6)$ & $C(5)$ & $P(5)$ & $C(6)$ & $105.4(8)$ \\
\hline $\operatorname{Pt}(1)$ & $P(1)$ & $C(121)$ & $114.2(6)$ & $C(5)$ & $P(5)$ & $C(511)$ & $102.4(7)$ \\
\hline$C(3)$ & $\mathrm{P}(1)$ & $C(111)$ & 104.0(7) & $C(6)$ & $P(5)$ & $C(511)$ & 107.2(7) \\
\hline$C(3)$ & $\mathrm{P}(1)$ & $C(121)$ & $103.0(8)$ & $\operatorname{Pt}(3)$ & $\mathrm{P}(6)$ & $C(6)$ & $111.7(5)$ \\
\hline$C(111)$ & $\mathrm{P}(1)$ & $C(121)$ & 108.3(8) & $\operatorname{Pt}(3)$ & $P(6)$ & $C(611)$ & $115.6(6)$ \\
\hline $\operatorname{Pt}(2)$ & $\mathrm{P}(2)$ & $C(3)$ & $110.9(5)$ & $\operatorname{Pt}(3)$ & $\mathrm{P}(6)$ & $C(621)$ & $112.7(5)$ \\
\hline $\operatorname{Pt}(2)$ & $\mathrm{P}(2)$ & $C(4)$ & $112.7(5)$ & $C(6)$ & $P(6)$ & $C(611)$ & 105.9(7) \\
\hline$C(6)$ & $P(6)$ & $C(621)$ & $105.5(8)$ & $F(23)$ & $\mathrm{P}(12)$ & $F(24)$ & $94(1)$ \\
\hline$C(611)$ & $P(6)$ & $C(621)$ & 104.7(7) & $F(23)$ & $\mathrm{P}(12)$ & $F(25)$ & $88(1)$ \\
\hline $\mathrm{F}(11)$ & $\mathrm{P}(11)$ & $\mathrm{F}(12)$ & $83(2)$ & $F(23)$ & $\mathrm{P}(12)$ & $F(26)$ & $176(1)$ \\
\hline $\mathrm{F}(11)$ & $\mathrm{P}(11)$ & $F(13)$ & $100(2)$ & $\mathrm{F}(24)$ & $\mathrm{P}(12)$ & $F(25)$ & $85(1)$ \\
\hline $\mathrm{F}(11)$ & $\mathrm{P}(11)$ & $\mathrm{F}(14)$ & $76(2)$ & $\mathrm{F}(24)$ & $\mathrm{P}(12)$ & $F(26)$ & $89(1)$ \\
\hline $\mathrm{F}(11)$ & $\mathrm{P}(11)$ & $\mathrm{F}(15)$ & 173(2) & $F(25)$ & $\mathrm{P}(12)$ & $F(26)$ & $91(1)$ \\
\hline $\mathrm{F}(11)$ & $\mathrm{P}(11)$ & $\mathrm{F}(16)$ & $92(1)$ & $C(1)$ & $\mathrm{N}(1)$ & $C(31)$ & $174(2)$ \\
\hline $\mathrm{F}(12)$ & $\mathrm{P}(11)$ & $F(13)$ & $90(1)$ & $C(2)$ & $N(2)$ & $C(41)$ & $175(2)$ \\
\hline $\mathrm{F}(12)$ & $\mathrm{P}(11)$ & $\mathrm{F}(14)$ & $93(1)$ & $\operatorname{Pt}(2)$ & $\mathrm{N}(11)$ & $\operatorname{Pt}(3)$ & $98.9(6)$ \\
\hline $\mathrm{F}(12)$ & $\mathrm{P}(11)$ & $F(15)$ & 99(2) & $\operatorname{Pt}(2)$ & $\mathrm{N}(11)$ & $C(11)$ & $138(1)$ \\
\hline $\mathrm{F}(12)$ & $\mathrm{P}(11)$ & $F(16)$ & $175(1)$ & $\operatorname{Pt}(3)$ & $\mathrm{N}(11)$ & $C(11)$ & $123(1)$ \\
\hline$F(13)$ & $\mathrm{P}(11)$ & $\mathrm{F}(14)$ & $174(2)$ & $\operatorname{Pt}(1)$ & $C(1)$ & $\mathrm{N}(1)$ & $173(2)$ \\
\hline $\mathrm{F}(13)$ & $\mathrm{P}(11)$ & $F(15)$ & $87(2)$ & $\operatorname{Pt}(3)$ & $C(2)$ & $N(2)$ & $174(1)$ \\
\hline $\mathrm{F}(13)$ & $\mathrm{P}(11)$ & $F(16)$ & $89(1)$ & $P(1)$ & $C(3)$ & $\mathrm{P}(2)$ & $105.2(8)$ \\
\hline $\mathrm{F}(14)$ & $\mathrm{P}(11)$ & $\mathrm{F}(15)$ & $97(2)$ & $\mathrm{P}(2)$ & $C(4)$ & $\mathrm{P}(3)$ & $115.6(9)$ \\
\hline $\mathrm{F}(14)$ & $\mathrm{P}(11)$ & $\mathrm{F}(16)$ & $88(1)$ & $\mathrm{P}(4)$ & $C(5)$ & $P(5)$ & $105.4(9)$ \\
\hline$F(15)$ & $\mathrm{P}(11)$ & $F(16)$ & $86(2)$ & $P(5)$ & $C(6)$ & $P(6)$ & $113.4(9)$ \\
\hline $\mathrm{F}(21)$ & $\mathrm{P}(12)$ & $\mathrm{F}(22)$ & $92(1)$ & $\mathrm{N}(11)$ & $C(11)$ & $C(12)$ & $129(2)$ \\
\hline $\mathrm{F}(21)$ & $\mathrm{P}(12)$ & $\mathrm{F}(23)$ & $93(1)$ & $\mathrm{N}(11)$ & $C(11)$ & $C(15)$ & $123(2)$ \\
\hline$F(21)$ & $\mathrm{P}(12)$ & $F(24)$ & $92(1)$ & $C(12)$ & $C(11)$ & $C(15)$ & 108(1) \\
\hline $\mathrm{F}(21)$ & $\mathrm{P}(12)$ & $F(25)$ & 177(1) & $C(11)$ & $C(12)$ & $C(13)$ & $110(1)$ \\
\hline $\mathrm{F}(21)$ & $\mathrm{P}(12)$ & $\mathrm{F}(26)$ & $88(1)$ & $\mathrm{C}(11)$ & $C(12)$ & $C(21)$ & $127(2)$ \\
\hline$F(22)$ & $\mathrm{P}(12)$ & $F(23)$ & 91(1) & $C(13)$ & $C(12)$ & $C(21)$ & $123(2)$ \\
\hline $\mathrm{F}(22)$ & $\mathrm{P}(12)$ & $F(24)$ & $173(1)$ & $C(12)$ & $C(13)$ & $C(14)$ & $103(2)$ \\
\hline $\mathrm{F}(22)$ & $\mathrm{P}(12)$ & $\mathrm{F}(25)$ & $90(1)$ & $C(12)$ & $C(13)$ & $C(22)$ & $111(2)$ \\
\hline $\mathrm{F}(22)$ & $\mathrm{P}(12)$ & $F(26)$ & $86(1)$ & $C(12)$ & $C(13)$ & $C(23)$ & $112(2)$ \\
\hline$C(14)$ & $C(13)$ & $C(22)$ & $112(2)$ & $C(31)$ & $C(32)$ & $C(37)$ & $123(2)$ \\
\hline$C(14)$ & $C(13)$ & $C(23)$ & $109(2)$ & $C(33)$ & $C(32)$ & $C(37)$ & $125(3)$ \\
\hline$C(22)$ & $C(13)$ & $C(23)$ & $110(2)$ & $C(32)$ & $C(33)$ & $C(34)$ & $117(3)$ \\
\hline$C(13)$ & $C(14)$ & $C(15)$ & $102(2)$ & $C(33)$ & $C(34)$ & $C(35)$ & $127(3)$ \\
\hline$C(13)$ & $C(14)$ & $C(24)$ & $108(2)$ & $C(34)$ & $C(35)$ & $C(36)$ & 117(3) \\
\hline$C(13)$ & $C(14)$ & $C(25)$ & $108(2)$ & $C(31)$ & $C(36)$ & $C(35)$ & 121(3) \\
\hline$C(15)$ & $C(14)$ & $C(24)$ & $118(2)$ & $C(31)$ & $C(36)$ & $C(38)$ & $119(2)$ \\
\hline$C(15)$ & $C(14)$ & $C(25)$ & 111(2) & $C(35)$ & $C(36)$ & $C(38)$ & 120(3) \\
\hline$C(24)$ & $C(14)$ & $C(25)$ & $110(2)$ & $\mathrm{N}(2)$ & $C(41)$ & $C(42)$ & $118(2)$ \\
\hline$C(11)$ & C(15) & $C(14)$ & 105(1) & $\mathrm{N}(2)$ & $C(41)$ & $C(46)$ & $115(2)$ \\
\hline$C(11)$ & $C(15)$ & $C(26)$ & $112(2)$ & $C(42)$ & $C(41)$ & $C(46)$ & $127(2)$ \\
\hline$C(11)$ & $C(15)$ & C(27) & 111(1) & $C(41)$ & $C(42)$ & $C(43)$ & $115(2)$ \\
\hline$C(14)$ & $C(15)$ & $C(26)$ & $106(1)$ & $C(41)$ & $C(42)$ & $C(47)$ & $124(2)$ \\
\hline$C(14)$ & $C(15)$ & $C(27)$ & $113(2)$ & $C(43)$ & $C(42)$ & $C(47)$ & $121(2)$ \\
\hline$C(26)$ & $C(15)$ & $C(27)$ & $110(2)$ & $C(42)$ & $C(43)$ & $C(44)$ & $122(2)$ \\
\hline $\mathrm{N}(21)$ & $C(21)$ & $C(12)$ & $174(2)$ & $C(43)$ & $C(44)$ & $C(45)$ & $121(2)$ \\
\hline $\mathrm{N}(22)$ & $C(22)$ & $C(13)$ & $176(2)$ & $\mathrm{C}(44)$ & $C(45)$ & $C(46)$ & $123(2)$ \\
\hline $\mathrm{N}(23)$ & $C(23)$ & $C(13)$ & $174(2)$ & $C(41)$ & $C(46)$ & $C(45)$ & 111(2) \\
\hline $\mathrm{N}(24)$ & $C(24)$ & $C(14)$ & $173(2)$ & $C(41)$ & $C(46)$ & $C(48)$ & $122(2)$ \\
\hline
\end{tabular}




\begin{tabular}{|c|c|c|c|c|c|c|c|}
\hline $\mathrm{N}(25)$ & $C(25)$ & $C(14)$ & $175(3)$ & $C(45)$ & $C(46)$ & $C(48)$ & $127(2)$ \\
\hline $\mathrm{N}(26)$ & $C(26)$ & $C(15)$ & $174(2)$ & $P(1)$ & $C(111)$ & $C(112)$ & 119(1) \\
\hline $\mathrm{N}(27)$ & $C(27)$ & $C(15)$ & $172(2)$ & $\mathrm{P}(1)$ & $C(111)$ & $C(116)$ & $120(1)$ \\
\hline $\mathrm{N}(1)$ & $C(31)$ & $C(32)$ & $117(2)$ & $C(112)$ & $C(111)$ & $C(116)$ & $121(2)$ \\
\hline $\mathrm{N}(1)$ & $C(31)$ & $C(36)$ & $117(2)$ & $C(111)$ & $C(112)$ & $C(113)$ & $120(2)$ \\
\hline$C(32)$ & $C(31)$ & $C(36)$ & $126(2)$ & $C(112)$ & $C(113)$ & $C(114)$ & $120(2)$ \\
\hline$C(31)$ & $C(32)$ & $C(33)$ & 112(3) & $C(113)$ & $C(114)$ & $C(115)$ & $119(2)$ \\
\hline$C(114)$ & $C(115)$ & $C(116)$ & $121(2)$ & $P(3)$ & $C(321)$ & $C(322)$ & $117(1)$ \\
\hline$C(111)$ & $C(116)$ & $C(115)$ & $119(2)$ & $P(3)$ & $C(321)$ & $C(326)$ & $120(1)$ \\
\hline $\mathrm{P}(1)$ & $C(121)$ & $C(122)$ & $120(2)$ & $C(322)$ & $C(321)$ & $C(326)$ & $122(2)$ \\
\hline $\mathrm{P}(1)$ & $C(121)$ & $C(126)$ & $121(1)$ & $C(321)$ & $C(322)$ & $C(323)$ & $118(2)$ \\
\hline$C(122)$ & $C(121)$ & $C(126)$ & $119(2)$ & $C(322)$ & $C(323)$ & $C(324)$ & 118(2) \\
\hline$C(121)$ & $C(122)$ & $C(123)$ & $116(2)$ & $C(323)$ & $C(324)$ & $C(325)$ & $126(2)$ \\
\hline$C(122)$ & $C(123)$ & $C(124)$ & $123(3)$ & $C(324)$ & $C(325)$ & $C(326)$ & $115(2)$ \\
\hline$C(123)$ & $C(124)$ & $C(125)$ & $122(2)$ & $C(321)$ & $C(326)$ & $C(325)$ & $121(2)$ \\
\hline$C(124)$ & $C(125)$ & $C(126)$ & $115(2)$ & $\mathrm{P}(4)$ & $C(411)$ & $C(412)$ & 119(1) \\
\hline$C(121)$ & $C(126)$ & $C(125)$ & $125(2)$ & $\mathrm{P}(4)$ & $C(411)$ & $C(416)$ & 119(1) \\
\hline$P(2)$ & $C(211)$ & $C(212)$ & 121(1) & $C(412)$ & $C(411)$ & $C(416)$ & $121(2)$ \\
\hline $\mathrm{P}(2)$ & $C(211)$ & $C(216)$ & 118(1) & $C(411)$ & $C(412)$ & $C(413)$ & $118(2)$ \\
\hline$C(212)$ & $C(211)$ & $C(216)$ & $121(2)$ & $C(412)$ & $C(413)$ & $C(414)$ & $121(2)$ \\
\hline$C(211)$ & $C(212)$ & $C(213)$ & $118(2)$ & $C(413)$ & $C(414)$ & $C(415)$ & $120(2)$ \\
\hline$C(212)$ & $C(213)$ & $C(214)$ & $122(2)$ & $C(414)$ & $C(415)$ & $C(416)$ & $120(2)$ \\
\hline$C(213)$ & $C(214)$ & $C(215)$ & $119(2)$ & $C(411)$ & $C(416)$ & $C(415)$ & $120(2)$ \\
\hline$C(214)$ & $C(215)$ & $C(216)$ & $119(2)$ & $\mathrm{P}(4)$ & $C(421)$ & $C(422)$ & 119(1) \\
\hline$C(211)$ & $C(216)$ & $C(215)$ & $120(2)$ & $\mathrm{P}(4)$ & $C(421)$ & $C(426)$ & 121(1) \\
\hline $\mathrm{P}(3)$ & $C(311)$ & $C(312)$ & 120(1) & $C(422)$ & $C(421)$ & $C(426)$ & 119(2) \\
\hline $\mathrm{P}(3)$ & $C(311)$ & $C(316)$ & 121(1) & $C(421)$ & $C(422)$ & $C(423)$ & $121(2)$ \\
\hline$C(312)$ & $C(311)$ & $C(316)$ & $119(2)$ & $C(422)$ & $C(423)$ & $C(424)$ & $116(2)$ \\
\hline$C(311)$ & $C(312)$ & $C(313)$ & $120(2)$ & $C(423)$ & $C(424)$ & $C(425)$ & $124(2)$ \\
\hline$C(312)$ & $C(313)$ & $C(314)$ & $122(2)$ & $C(424)$ & $C(425)$ & $C(426)$ & $120(2)$ \\
\hline$C(313)$ & $C(314)$ & $C(315)$ & $121(2)$ & $C(421)$ & $C(426)$ & $C(425)$ & $119(2)$ \\
\hline$C(314)$ & $C(315)$ & $C(316)$ & $119(2)$ & $P(5)$ & $C(511)$ & $C(512)$ & 121(1) \\
\hline$C(311)$ & $C(316)$ & $C(315)$ & $119(2)$ & $P(5)$ & $C(511)$ & $C(516)$ & 119(1) \\
\hline$C(512)$ & $C(511)$ & $C(516)$ & $120(2)$ & $\mathrm{O}(702)$ & $C(721)$ & $C(723)$ & $99(5)$ \\
\hline$C(511)$ & $C(512)$ & $C(513)$ & $122(2)$ & $C(722)$ & $C(721)$ & $C(723)$ & $136(7)$ \\
\hline$C(512)$ & $C(513)$ & $C(514)$ & $118(2)$ & & & & \\
\hline$C(513)$ & $C(514)$ & $C(515)$ & $122(2)$ & & & & \\
\hline$C(514)$ & $C(515)$ & $C(516)$ & $117(2)$ & & & & \\
\hline$C(511)$ & $C(516)$ & $C(515)$ & $120(2)$ & & & & \\
\hline$P(6)$ & $C(611)$ & $C(612)$ & $122(1)$ & & & & \\
\hline $\mathrm{P}(6)$ & $C(611)$ & $C(616)$ & $119(1)$ & & & & \\
\hline$C(612)$ & $C(611)$ & $C(616)$ & $119(2)$ & & & & \\
\hline$C(611)$ & $C(612)$ & $C(613)$ & $120(2)$ & & & & \\
\hline$C(612)$ & $C(613)$ & $C(614)$ & $119(2)$ & & & & \\
\hline$C(613)$ & $C(614)$ & $C(615)$ & $123(2)$ & & & & \\
\hline$C(614)$ & $C(615)$ & $C(616)$ & $117(2)$ & & & & \\
\hline$C(611)$ & $C(616)$ & $C(615)$ & $121(2)$ & & & & \\
\hline $\mathrm{P}(6)$ & $C(621)$ & $C(622)$ & 115(1) & & & & \\
\hline$P(6)$ & $C(621)$ & $C(626)$ & $124(1)$ & & & & \\
\hline$C(622)$ & $C(621)$ & $C(626)$ & $121(2)$ & & & & \\
\hline$C(621)$ & $C(622)$ & $C(623)$ & $117(2)$ & & & & \\
\hline$C(622)$ & $C(623)$ & $C(624)$ & $124(2)$ & & & & \\
\hline$C(623)$ & $C(624)$ & $C(625)$ & $118(2)$ & & & & \\
\hline$C(624)$ & $C(625)$ & $C(626)$ & $120(2)$ & & & & \\
\hline$C(621)$ & $C(626)$ & $C(625)$ & $121(2)$ & & & & \\
\hline $\mathrm{O}(701)$ & $C(711)$ & $C(712)$ & 115(3) & & & & \\
\hline
\end{tabular}


$\mathrm{O}(701) \quad \mathrm{C}(711) \quad \mathrm{C}(713) \quad 124(4)$

$\mathrm{C}(712) \quad \mathrm{C}(711) \quad \mathrm{C}(713) \quad 120(4)$

$\mathrm{O}(702) \quad \mathrm{C}(721) \quad \mathrm{C}(722) \quad 114(7)$

a Estimated deviations are given in parentheses. 
Figure S1. ORTEP plot for the complex cation of 2, $\left[\mathrm{Pt}_{3}(\mu \text {-dpmp })_{2}(\mathrm{MesNC})_{2}\right]\left(\mathrm{PF}_{6}\right)_{2}$.

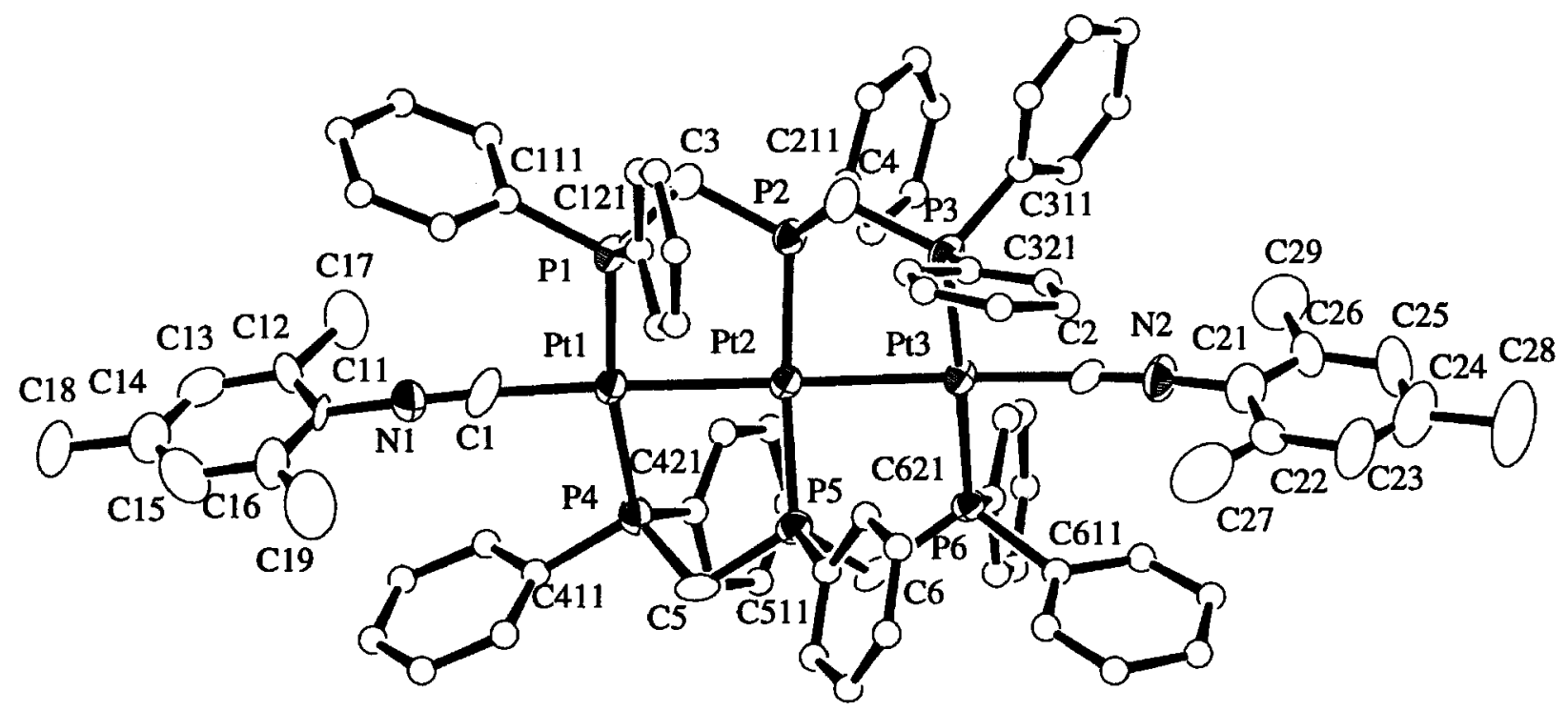

Figure S2. ORTEP plot for the complex cation of 4 , $\left[\mathrm{Pt}_{3}(\mu-\mathrm{dpmp})_{2}(t-\mathrm{BuNC})_{2}\right]\left(\mathrm{PF}_{6}\right)_{2}$.

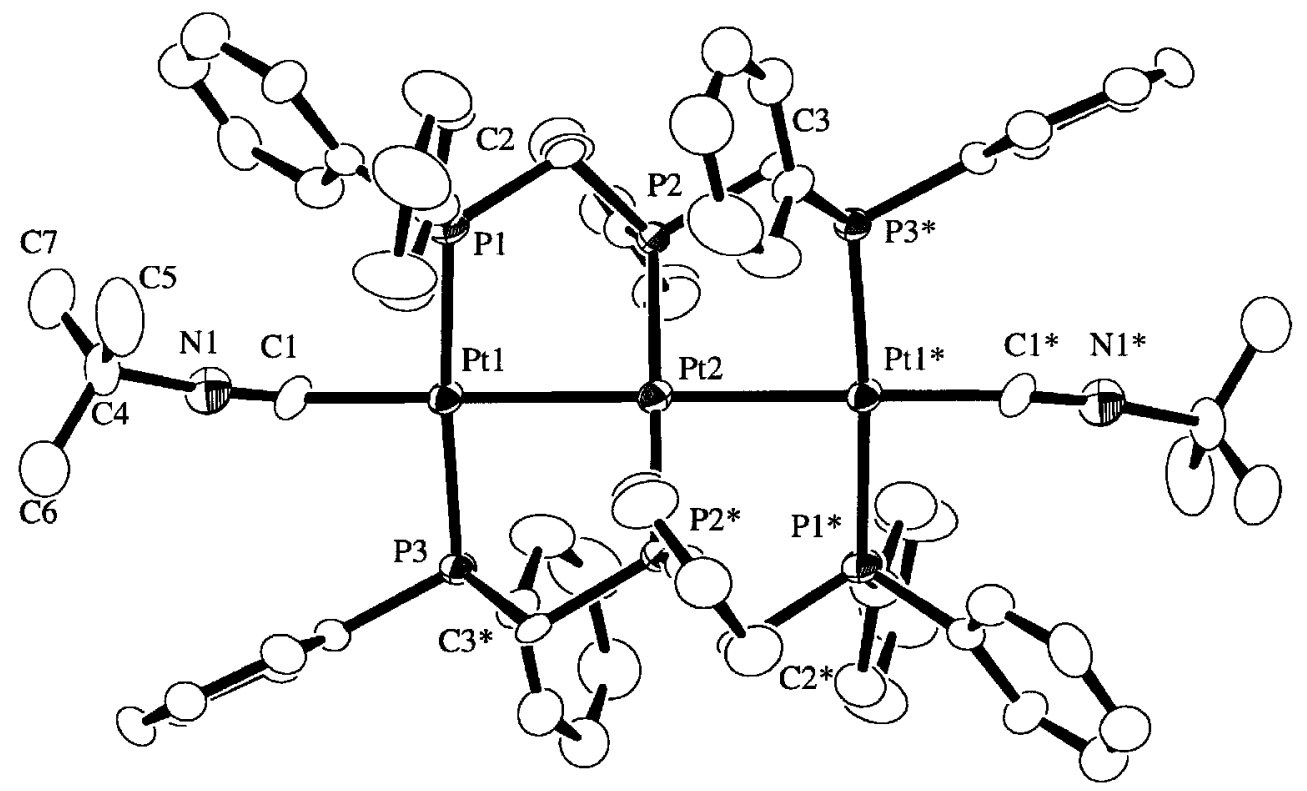


Figure S3-1. Raw EXAFS data, $k^{3} \chi(k)$
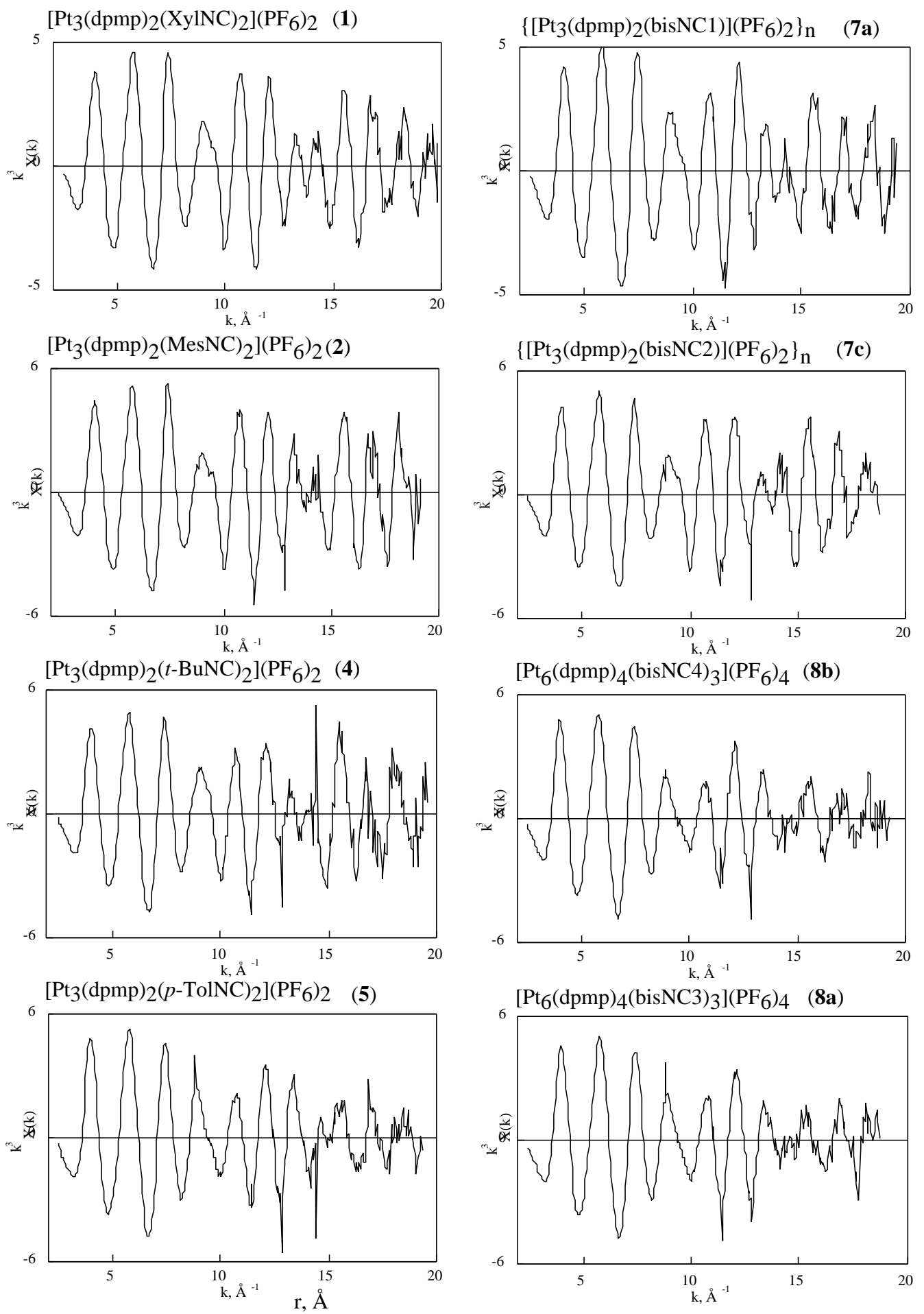

Figure S3-1. Raw EXAFS oscillation $\boldsymbol{k}^{3} \chi(\boldsymbol{k})$. 
Figure S3-2 Fourier Transforms of $k^{3} \chi(k)$
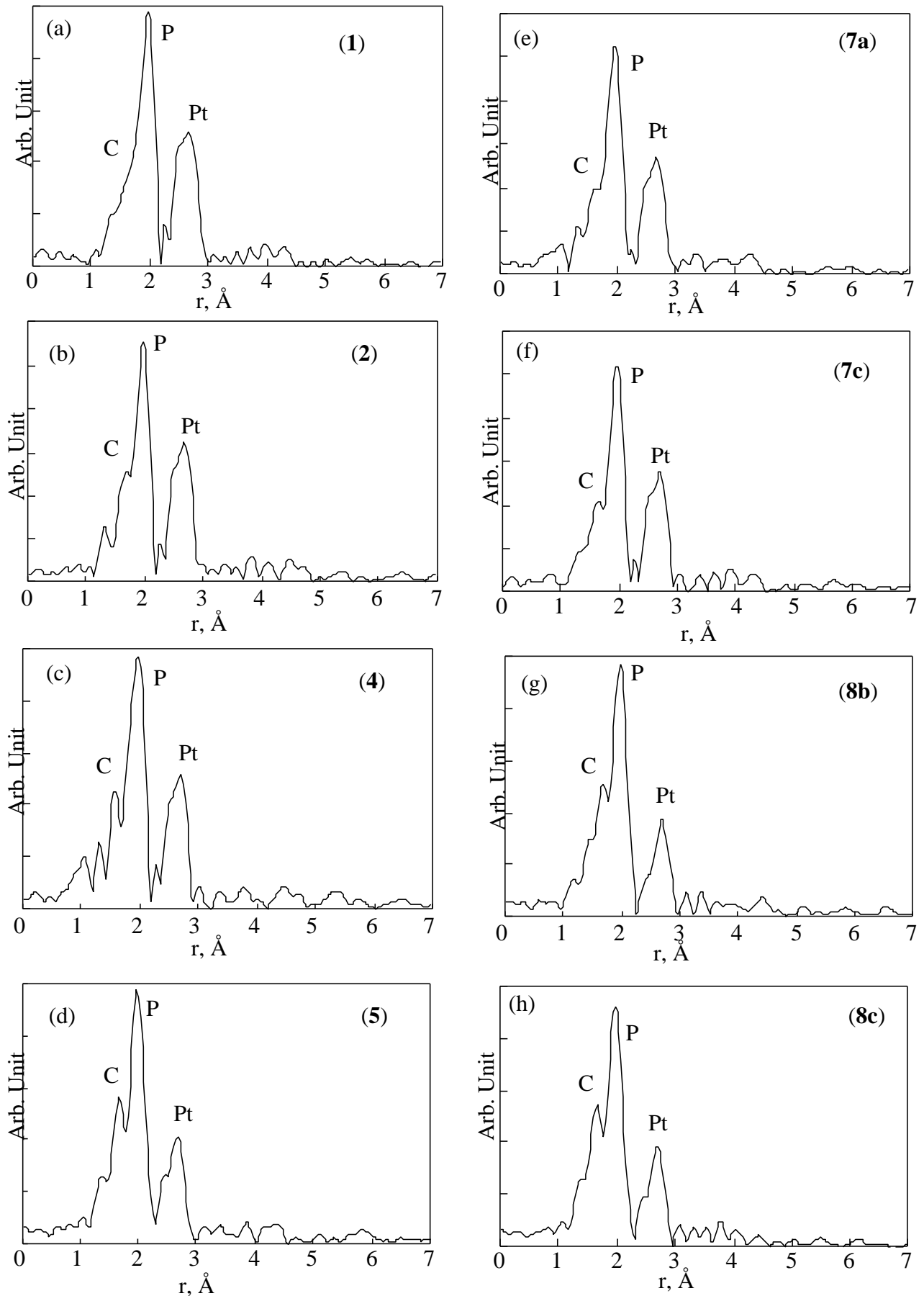

Figure S3-2. Fourier transforms of $\boldsymbol{k}^{3} \chi(\boldsymbol{k})$ for the powdered samples of (a) 1, (b) 2, (c) 4, (d) $\mathbf{5}$, (e) $7 \mathbf{a}$, (f) $7 \mathbf{c}$, (g) $8 \mathbf{b}$, and (h) $8 \mathbf{a}$. 
Figure S3-3 Curve-fitting Results

(a)

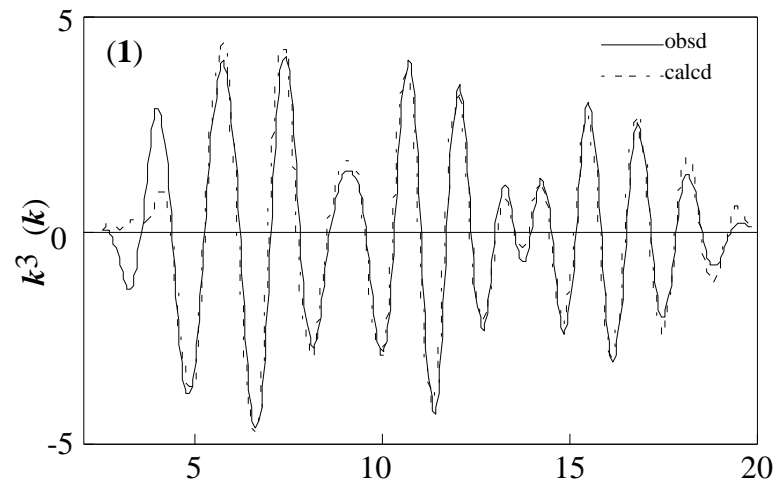

(b)

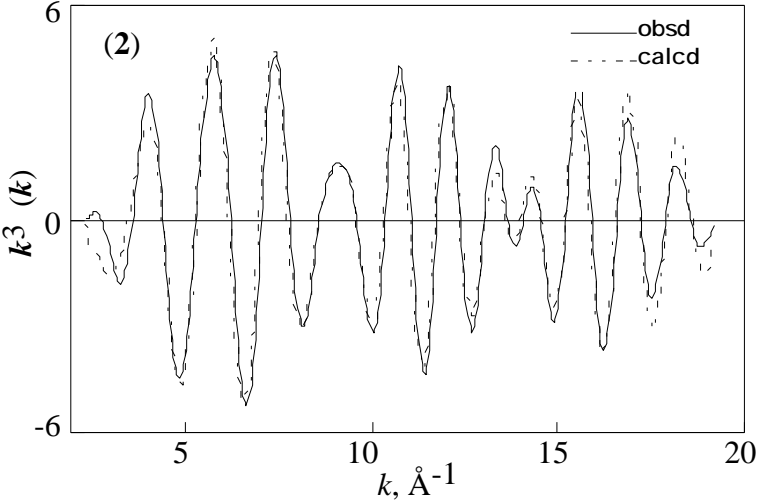

(e)

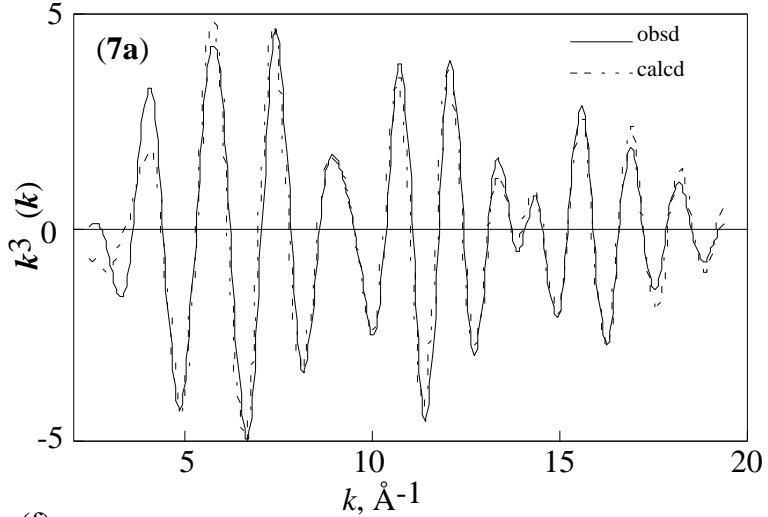

(f)

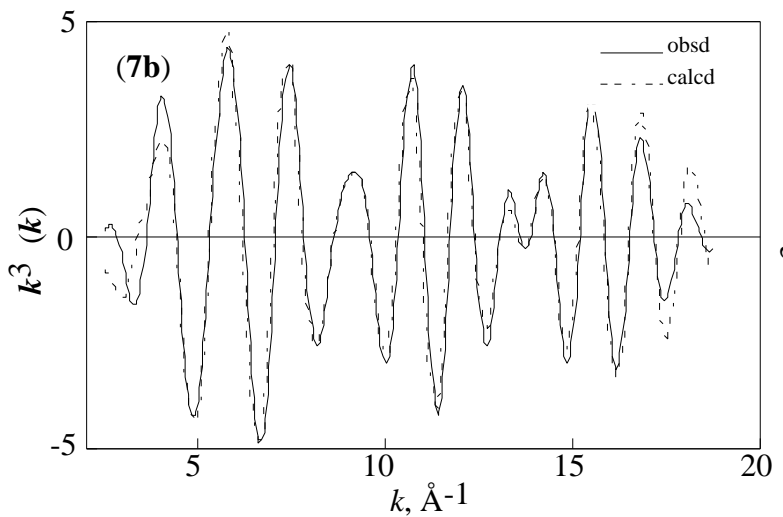

(c)

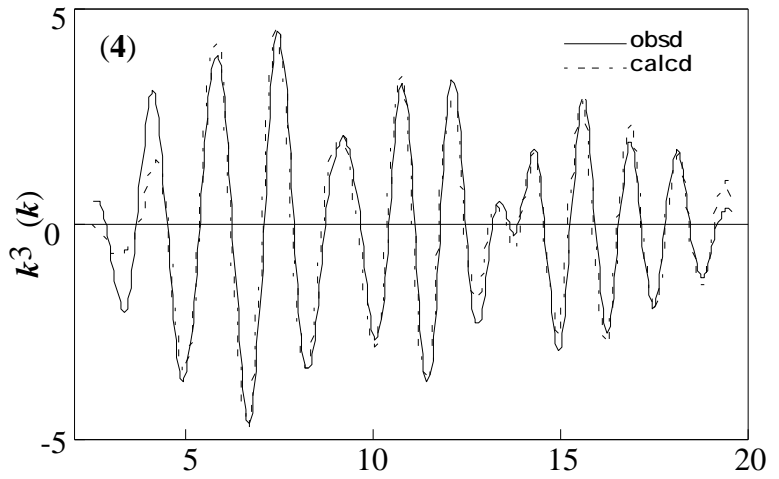

(d)

$k, \AA^{-1}$

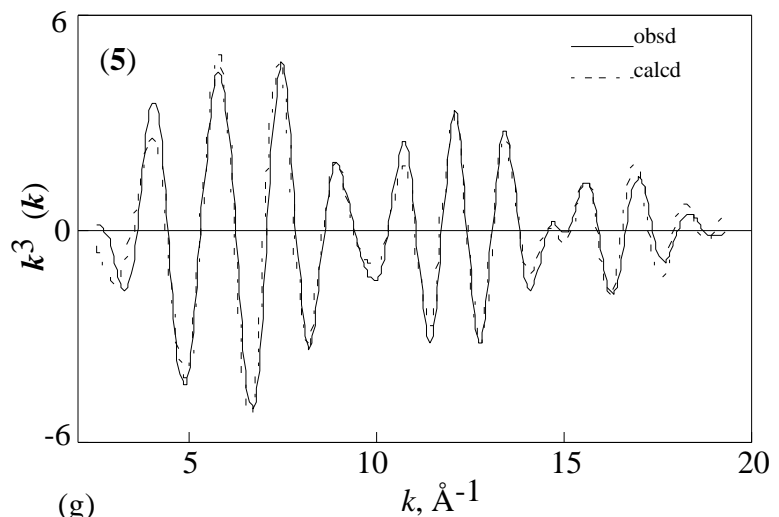

(g)

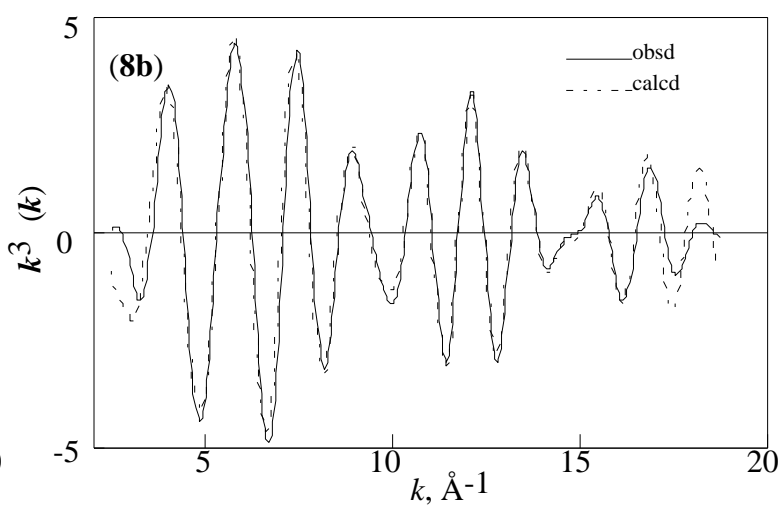

(h)

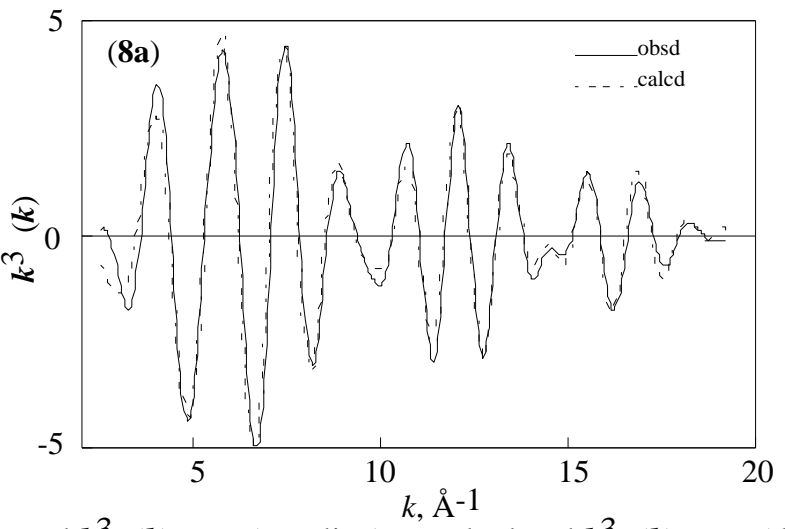

Figure S3. Curve-fitting results, Fourier filtered observed $\boldsymbol{k}^{3} \chi(\boldsymbol{k})_{\text {obsd }}$ (true line) vs calculated $\boldsymbol{k}^{3} \chi(\boldsymbol{k})_{\text {calcd }}$ (dotted line) for the powdered samples of (a) $\mathbf{1}$, (b) $\mathbf{2}$, (c) $\mathbf{4}$, (d) $\mathbf{5}$, (e) 7a, (f) 7c, (g) 8b, and (h) $\mathbf{8 a}$. 
Figure S4. UV-Vis spectra of complexes 1-5 in dichloromethane.

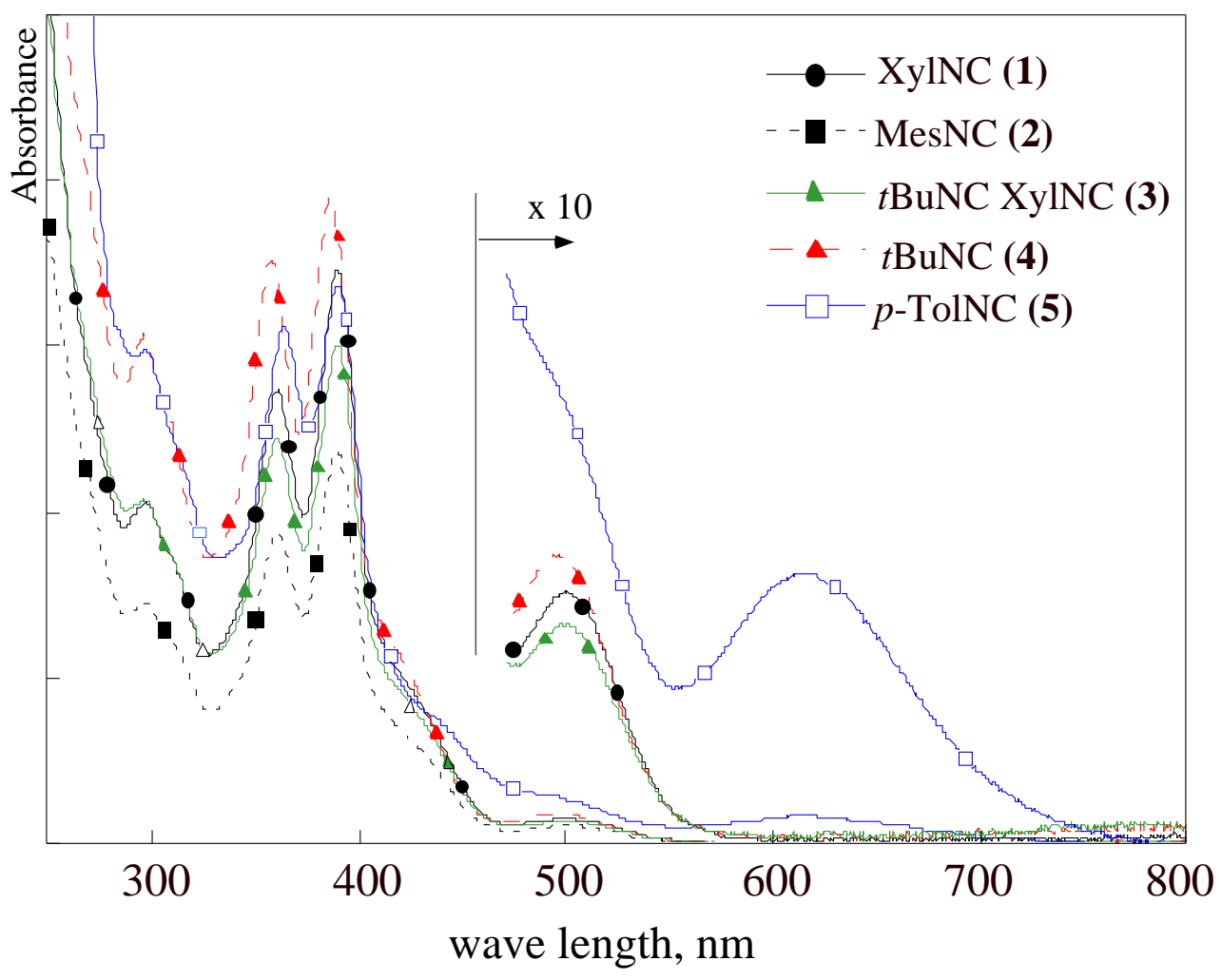


Figure S5. ORTEP plot for complex $6 \mathbf{a},\left[\mathrm{Pt}_{3}(\mu-\mathrm{dpmp})_{2}(\mathrm{XylNC})_{2}\right] \mathrm{I}_{2}$.

( I1

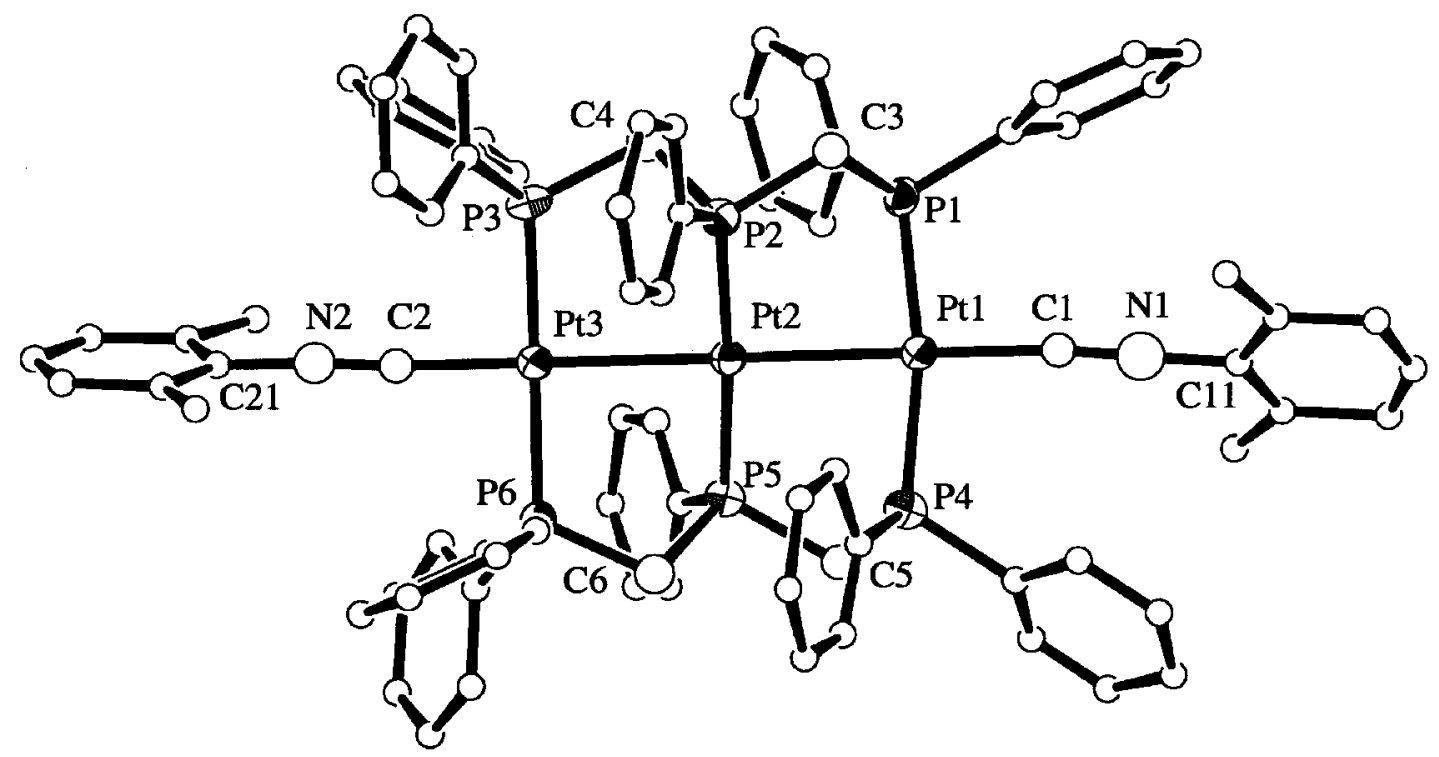

(12

Figure S6. ORTEP plot for complex $6 \mathbf{c},\left[\mathrm{Pt}_{3}(\mu-\mathrm{dpmp})_{2}(\mathrm{XylNC})_{2}\right]\left[\mathrm{Pt}(\mathrm{CN})_{4}\right]$.

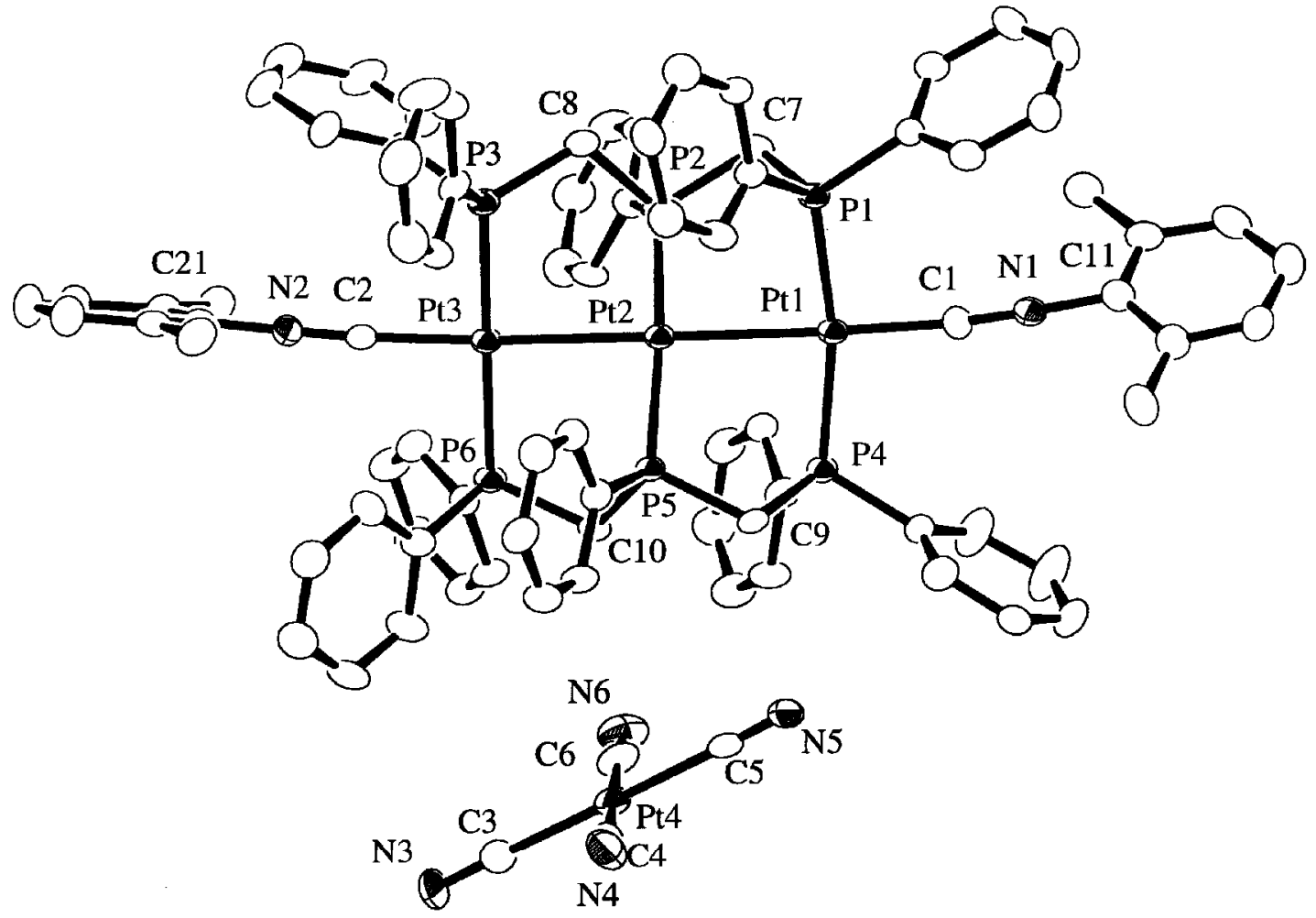


Figure S7. $\mathrm{MO}$ interaction diagram for $\left[\mathrm{Pt}_{3}(\mu-\mathrm{NO})_{2}\left(\mathrm{PH}_{3}\right)_{6}(\mathrm{CNH})_{2}\right]^{4+}(\mathbf{C})$ from the fragments $\left[\mathrm{Pt}_{3}\left(\mathrm{PH}_{3}\right)_{6}(\mathrm{CNH})_{2}\right]^{2+}(\mathrm{C} 1)$ and $\left[(\mathrm{NO})_{2}\right]^{2+}(\mathrm{C} 2)$ on the basis of EHMO calculations and $\mathrm{CACAO}$ fragment analysis.

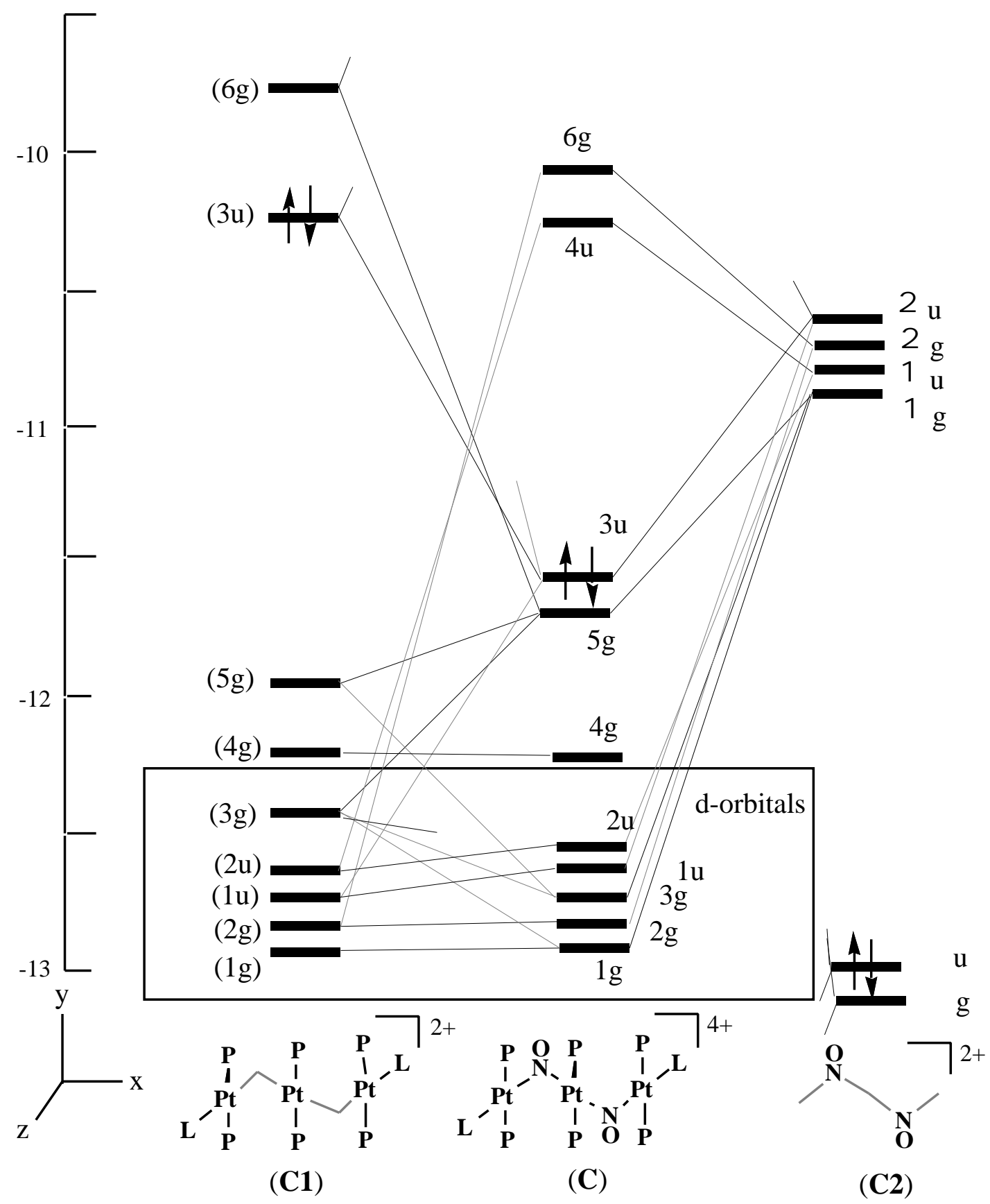


Figure S8. Electronic absorption spectra of the polymer $\mathbf{1 3}$ (- circle-) and the polymer 13 and excess of $\mathrm{Et}_{3} \mathrm{~N}$ (-black square-) in acetonitrile at room temperature; the latter is closely similar to that of $\mathbf{7 a}$.

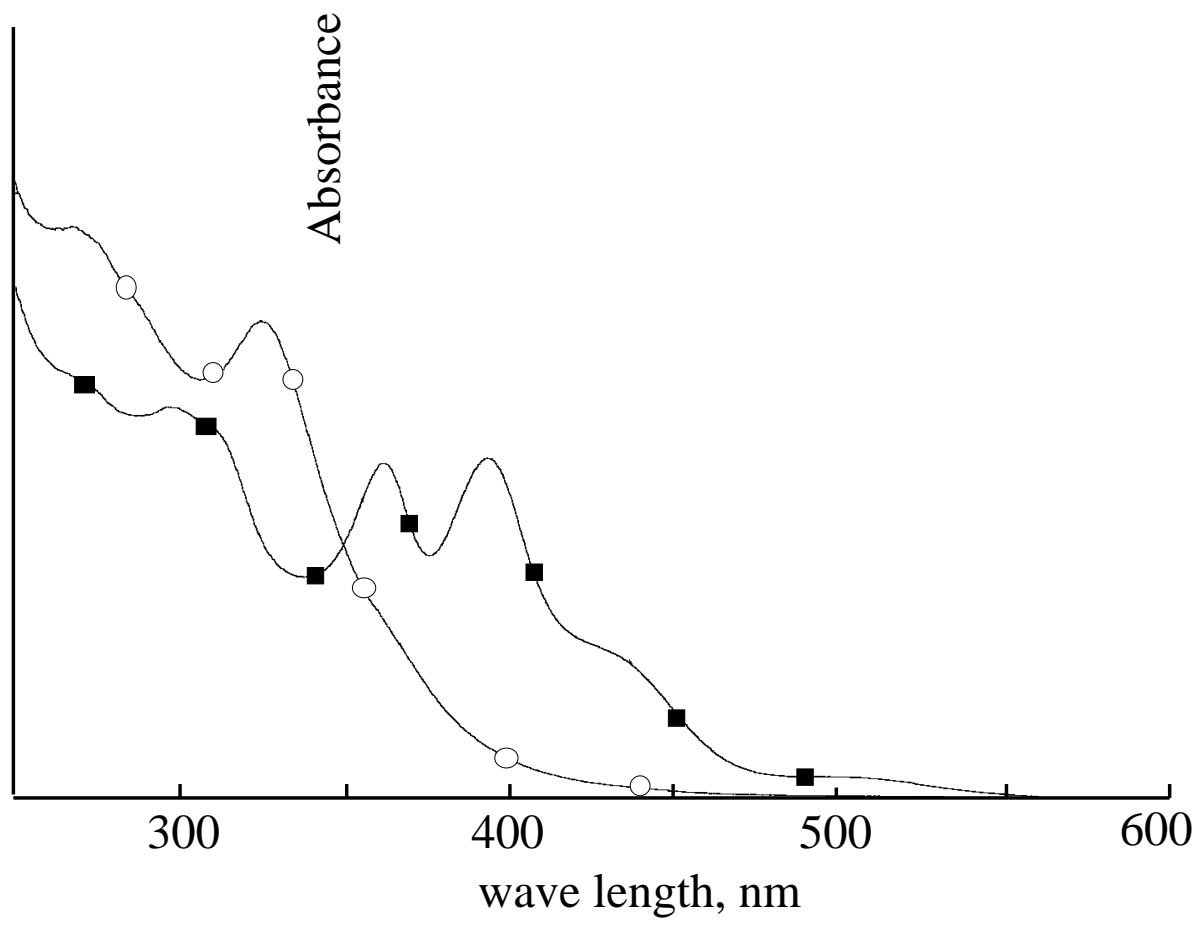

Equation 1. Theoretical expression of EXAFS oscillation.

$$
k^{3} \chi(k)=\sum_{\mathrm{i}}\left(\frac{k^{2} N_{\mathrm{i}}}{r_{\mathrm{i}}^{2}} S_{\mathrm{i}} F_{\mathrm{i}}(k) \exp \left(-2 \sigma_{\mathrm{i}}^{2} k^{2}\right) \sin \left(2 k r_{\mathrm{i}}+\Phi_{\mathrm{i}}(k)\right)\right)
$$

